UCRL-ID-129830

\title{
First Increment of Second Specimen Results to Performance Assessment-Long Term Corrosion
}

Francis Wang

January 1998

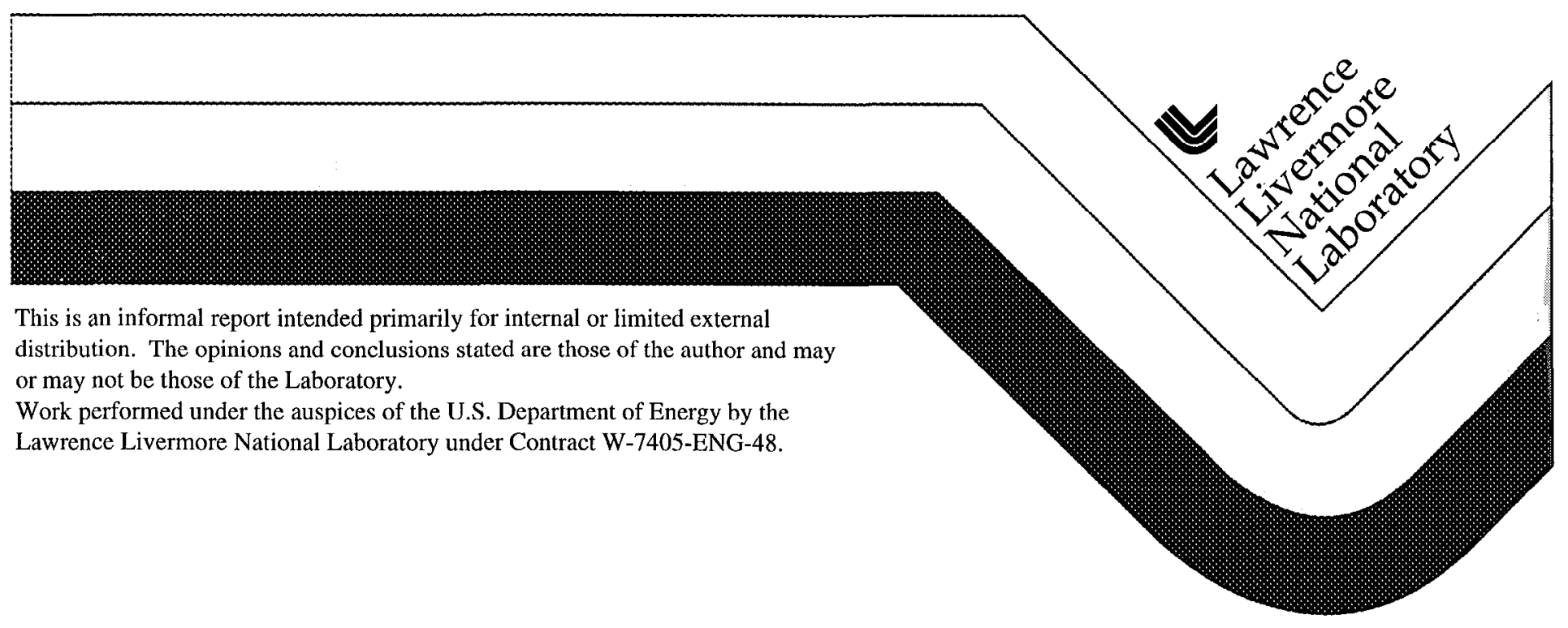




\section{DISCLAIMER}

This document was prepared as an account of work sponsored by an agency of the United States Government Neither the United States Government nor the University of California nor any of their employees, makes any warranty, express or implied, or assumes any legal liability or responsibility for the accuracy, completeness, or usefulness of any information, apparatus, product, or process disclosed, or represents that its use would not infringe privately owned rights Reference herein to any specific commercial product, process, or service by trade name, trademark, manufacturer, or otherwise, does not necessarily constitute or imply its endorsement, recommendation, or favoring by the United States Government or the University of California. The views and opinions of authors expressed herein do not necessarily state or reflect those of the United States Government or the University of California and shall not be used for advertising or product endorsement purposes

This report has been reproduced directly from the best available copy

Available to DOE and DOE contractors from the

Office of Scientific and Technical Information

PO Box 62, Oak Ridge, TN 37831

Prices available from (423) 576-8401

Available to the public from the National Technical Information Service

US Department of Commerce 5285 Port Royal Rd, Springfield, VA 22161 
First Increment of Second Specimen Results to Performance Assessment - Long Term Corrosion

Milestone WP601BM4

Francis Wang January 1998 
In this correspondence, we report the 6 month corrosion data of corrosion resistant alloys (alloys 825, G3, C22 \& C4, and Ti Gr 12 \& Ti Gr 16, and Inconel 625) and one year data of corrosion-allowance materials (A387 Gr22, A516 Gr55 and A27 Gr70-40), intermediate resistance alloys (Monel 400 and CDA 715) and corrosion resistant materials (alloys C22 and 625 only).

Currently there are 24 vessels in the Long Term Corrosion Studies Facility. Four types of solutions are used for the corrosion study. They are: (1) basic (from bicarbonate) simulated dilute water (SDW), (2) basic simulated concentrated water (SCW), (3) acidic concentrated solutions (SAW) which contain no bicarbonate and fluoride and (4) Simulated Cement Modified Water (SCMW). Studies conducted at $60^{\circ} \mathrm{C}$ and $90^{\circ} \mathrm{C}$. The base-line compositions of each solution are shown in the following table: (all concentrations are in $\mathrm{ppm})$.

\begin{tabular}{|c|c|c|c|c|c|c|c|c|c|c|c|c|}
\hline Solution & $\mathrm{T} \mathrm{C}^{\circ}$ & $\mathrm{pH}$ & $\underline{\mathbf{C a}^{2+}}$ & $\mathbf{M g}^{2+}$ & $\underline{\mathrm{K}^{+}}$ & $\underline{\mathrm{Na}^{+}}$ & $\underline{S_{i *}^{*}}$ & $\mathrm{SO}_{1}^{2-}$ & $\underline{\mathrm{Cl}^{-}}$ & $\mathrm{NO}_{3}$ & $\mathbf{F}^{*}$ & $\underline{\mathrm{HCO}_{3}}$ \\
\hline $\begin{array}{l}\text { SDW } \\
\text { SDW }\end{array}$ & $\begin{array}{l}60 \\
90\end{array}$ & 9.5 & $\begin{array}{l}3.5 \\
3.4\end{array}$ & $\begin{array}{l}1.2 \\
\text { ND }\end{array}$ & $\begin{array}{l}36 \\
38\end{array}$ & $\begin{array}{l}430 \\
460\end{array}$ & $\begin{array}{l}17 \\
16\end{array}$ & $\begin{array}{l}170 \\
180\end{array}$ & $\begin{array}{l}68 \\
74\end{array}$ & $\begin{array}{l}62 \\
64\end{array}$ & $\begin{array}{l}14 \\
15\end{array}$ & $\begin{array}{l}720 \\
700\end{array}$ \\
\hline $\begin{array}{l}\text { SCW } \\
\text { SCW }\end{array}$ & $\begin{array}{l}60 \\
90\end{array}$ & $\begin{array}{l}9.2 \\
9.2\end{array}$ & $\begin{array}{l}16 \\
15\end{array}$ & $\begin{array}{l}29 \\
3.4\end{array}$ & & & $\begin{array}{l}18 \\
58\end{array}$ & & & & & \\
\hline $\begin{array}{l}\text { SAW } \\
\text { SAW }\end{array}$ & $\begin{array}{l}60 \\
90\end{array}$ & $\begin{array}{l}2.7 \\
2.7\end{array}$ & $\begin{array}{l}58 \\
58\end{array}$ & $\begin{array}{l}52 \\
53\end{array}$ & $\begin{array}{l}4,300 \\
4,300\end{array}$ & $\begin{array}{l}43,000 \\
43,000\end{array}$ & $\begin{array}{l}30 \\
50\end{array}$ & $\begin{array}{l}41, \\
40\end{array}$ & $\begin{array}{l}28,000 \\
27,000\end{array}$ & $\begin{array}{l}23,000 \\
24,000\end{array}$ & 0 & $\begin{array}{l}0 \\
0\end{array}$ \\
\hline SCMV & 60 & 7.8 & 400 & 4 & 85 & 10 & 10 & 1200 & 11 & 10 & $<0.1$ & $<1$ \\
\hline
\end{tabular}

*sodium metasilicate was used as the silicate, but it was analyzed by ICP as elemental silicon.

In general, the concentration of each ion in SCW should be $\sim 100$ times of that in SDW. However $\mathrm{Ca}^{2+}$ and $\mathrm{Mg}^{2+}$ form insoluble salts with $\mathrm{HCO}_{3}{ }^{-}, \mathrm{F}^{-}$and silicate.

In all the basic SCW and SDW solutions, pH values has increased slowly from 9 to 10 over the course of the year's testing. This is due to the decomposition of bicarbonate ions $\left(\mathrm{HCO}_{3}{ }^{-} \rightarrow \mathrm{CO}_{2}+\mathrm{OH}^{-}\right)$. The SAW solution used for the corrosion study of intermediate resistant materials can dissolve both copper and nickle and raise the pH from 2.7 to 5.3 in few weeks. To continue the corrosion study, it was decided to maintain the "equilibrium" $\mathrm{pH}$ value (5 to 6 ) and not to add more acid to the solution.

In each test vessel, approximately half of the specimens are submersed in the test solutions, about half are in the water vapor saturated air above the test solutions, and a limited number of specimens are at the waterline. The temperature of the vapor phase is 2 to $4^{\circ} \mathrm{C}$ lower then the solution phase, hence there is water condensation on the specimens in the vapor phase.

On the attached two sets of data sheets (Corrosion Rate Calculations for Weight Loss Specimens and Corrosion Rate Calculations for Crevice Specimens) the sample identification is composed of an alphanumeric matrix. The first, second, and third characters describe the alloy, specimen type, and metallurgical condition, respectively. The first character indicates alloys A through N. The alloy designation of each specimen is provided in a column on the data sheets. The second character indicates the specimen type, "W" for Weight Loss specimen and " $C$ " for Crevice specimen. The third character indicates the metallurgical condition A through J. A specimen with a third character identification " $A$ " indicates a non-welded specimen, while specimens with the third character identified with letters B through J indicate 
welding practices specific to each alloy system. The last three digits are sequential numeric characters that simply distinguish one sample from another only by the particular number. Weight loss specimens are nominally $2.54 \mathrm{~cm} \mathrm{x} 5.08 \mathrm{~cm}(1$ " $\mathrm{2}$ ") and crevice specimens are $5.08 \mathrm{~cm} \times 5.08 \mathrm{~cm}(2 " \times 2 ")$.

The results of corrosion rate are divided into three groups:

\section{Corrosion-allowance materials}

For both weight-loss and crevice specimens, visual inspection and weight loss data gave no clear evidence of a difference in the corrosion resistance of welded and unwelded areas. For the crevice specimens, the creviced area is only a small fraction of the total surface area. Majority of the creviced area is not attacked. The corrosion rates of crevice specimens was similar to that of the weight-loss specimens.

Because the corrosion of welded and unwelded weight-loss and crevice specimens are quite similar, we decide to group them together to obtain an average corrosion rate for each steel. The corrosion rates are shown in the attached Table 1. Each corrosion rate of waterline specimens is the average of 2, 1 unwelded and 1 welded weight-loss specimen. For in- and above-water specimens, each rate is an average of 12 specimens ( 3 unwelded weight-loss, 3 unwelded crevice, 3 welded weight-loss and 3 welded crevice).

The data presented in Table 1 underestimates the actual corrosion penetration into the specimens for two reasons. 1. In some cases, portions of a specimen were only lightly attacked, while other portions of the same specimen were significantly attacked. 2 . The depth of penetration was not uniform in the attacked area, that is the surface was roughened on a macroscopic scale. The roughening was primarily broad pits. More detailed study is in progress to determine the characteristics of the penetration front into the specimens.

As indicated in Table 1, the above-water specimens in vessels of SCW have higher corrosion rates than those of the in-water specimens. This is probably caused by the high $\mathrm{CO}_{2}$ concentration in the vapor phase, which is the results of the bicarbonate decomposition in solution. Carbon dioxide released to the vapor phase will be redissolved in the condensate on the surfaces of above-water specimens and forms carbonic acid which can promote the corrosion of iron. The lower corrosion rates of the at-waterline specimens in SCW at $90^{\circ} \mathrm{C}$, especially A516, may be because the samples were mounted vertically and less condensate collected on the surface. Also, at $90^{\circ} \mathrm{C}$ the solubility of $\mathrm{CO}_{2}$ in the condensate is less than that at $60{ }^{\circ} \mathrm{C}$.

A residual gas analyzer (RGA) was used to analyze the gas above the SCW at 60 and $90^{\circ} \mathrm{C}$. The $\mathrm{CO}_{2}$ content is $\sim 30$ times and $\sim 70$ times greater than that of normal atmosphere air respectively.

The concentration of dissolved oxygen in test solutions can be a factor for the corrosion rate of in-water specimens. The concentrations were measured and they are $7.0 \mathrm{ppm}$ in SDW and $5.2 \mathrm{ppm}$ in SCW at $24^{\circ} \mathrm{C}$. It is known that oxygen is less soluble in a solution with higher dissolved salts when compared at same temperature, but the difference in oxygen solubility among the solutions is less than that at a higher temperature. We expected that at either 60 or $90^{\circ} \mathrm{C}$, the difference of dissolved oxygen concentration between SDW and SCW is less than $1.8 \mathrm{ppm}(7.0-5.2=1.8)$. It is too small to explain the lower corrosion rates of in-water specimens of SCW than that of those of SDW, especially at $90^{\circ} \mathrm{C}$. 
The low corrosion rate of in-water specimens in SCW is likely due to the passivation of steels by the high concentration of anions, especially bicarbonate. Although anions can contribute to the destruction of the oxide film, at high concentration they can also initiate inhibition by being adsorbed on to the metal surface at weak points in the oxide film, suppressing anodic dissolution of the metal. Less aggressive anions are likely to be able to passivate at lower concentrations than the more aggressive ones. In the test solutions used for this study, bicarbonate is the least aggressive major anion.

For coupons mounted at the waterline, the corrosion condition of above water portion is similar to that of the above-water specimens; the under water portion is usually worse than that of in-water specimens. The worst corrosion is always at the part right below the waterline.

Using the x-ray diffraction method, we have identified the corrosion products. They are: $\mathrm{Fe}_{2} \mathrm{O}_{3}, \mathrm{FeO}(\mathrm{OH})$ and $\mathrm{Fe}_{3} \mathrm{O}_{4}$. No iron carbonates were found in the corrosion product collected from the worst corroded coupons (i.e. above-water of SCW at $90^{\circ} \mathrm{C}$ ).

U-bend specimens from the corrosion-allowance specimens in 60 and $90^{\circ} \mathrm{C}$ SDW and SCW environments exhibited no evidence of SCC under light microscopy examination.

\section{Intermediate-resistant materials}

A summary of the corrosion rate of Monel 400 and CDA 715 at 60 and $90^{\circ} \mathrm{C}$ is shown in the attached Table 2. Again, each corrosion rate of a specimen is the average of 12 specimens (3 unwelded weight-loss, 3 unwelded crevice, 3 welded weight-loss and 3 welded crevice). The large weight loss of in-water specimens after 6 month is due to acidic dissolution (i.e. $\mathrm{M}+2 \mathrm{H}^{+}=\mathrm{M}^{2+}+\mathrm{H}_{2}$ ). The acidic dissolution is indicated by the change of $\mathrm{pH}$ values from $\sim 3$ to $\sim 5$ in few weeks (i.e. equivalent to $\sim 0.5$ mole of divalent metals dissolved in 1,000 liters of solution). To continue the long term corrosion study of intermediate-resistant materials, we decided to allow the study to proceed under the "equilibrium" $\mathrm{pH}$ values (5 to 6). For the one year corrosion data, the solution $\mathrm{pH}$ values were kept at 5.3 .

No SCC was observed in U-bend specimens under light microscopy examination.

\section{Corrosion-resistant materials}

Summaries of the corrosion resistant material are shown in the attached Tables 3 and 4 . The SAW used in this study showed no pH change after 6 months. In SAW at $60^{\circ} \mathrm{C}$, corrosion rates for all corrosion-resistant metals are negligible. However $\mathrm{Ti} G r 12$ and $\mathrm{Gr} 16$ showed some surface oxidation. It was also observed that many small pits appeared on the edges of all titanium specimens. We believe they may be due to the embeded material (iron) from cutting during the specimen fabrication. This will be further examined ( $\mathrm{x}$-ray fluorescence may show the existence of iron). One alloy 825 in-water coupon showed few very tiny pits in the creviced area.

For the one year corrosion data, only alloy C22 and Inconel 625 in the vessel of SAW at $60^{\circ} \mathrm{C}$ were completed.

No SCC was observed on any U-bend specimens under light microscopy examination. 


\section{Table 1: Corrosion rate (micron/year) of steel under different environments}

steel type

A387

A516

Cast carbon steel

A387

A516

Cast carbon steel

A387

A516

Cast carbon steel

A387

A516

Cast carbon steel solution and

temperature environment

\begin{tabular}{cccc} 
in - water & \multicolumn{2}{c}{ above - water } & \multicolumn{2}{c}{ at - waterline } \\
$6 \mathrm{mo} \quad 1 \mathrm{yr}$ & $6 \mathrm{mo} \quad 1 \mathrm{yr}$ & $6 \mathrm{mo} \quad 1 \mathrm{yr}$
\end{tabular}

SDW, $60^{\circ} \mathrm{C}$

same

same

SDW, $90^{\circ} \mathrm{C}$

same

same

SCW, $60^{\circ} \mathrm{C}$

same

same

SCW, $90^{\circ} \mathrm{C}$

same

same

$\begin{array}{ll}45 & 91 \\ 93 & 76 \\ 94 & 78\end{array}$

$100 \quad 73$

$100 \quad 54$

$80 \quad 50$

$\begin{array}{ll}25 & 7\end{array}$

$66 \quad 10$

$72 \quad 11$

124

126

147

$\begin{array}{ll}31 & 18 \\ 46 & 27 \\ 51 & 37\end{array}$

36

$77 \quad 56$

$72 \quad 39$

$110 \quad 132$

$210 \quad 194$

$200 \quad 114$

$210 \quad 287$

$240 \quad 227$

$180 \quad 148$
$130 \quad 158$

$220 \quad 194$

$190 \quad 208$

$210 \quad 165$

$240 \quad 111$

$240 \quad 119$

$170 \quad 135$

$210 \quad 190$

$85 \quad 99$

$16 \quad 58$

$94 \quad 89$
$210 \quad 134$ 


\section{Table 2: Corrosion rate (micron/year) of Monel 400 and CDA 715 at $60^{\circ} \mathrm{C} \& 90^{\circ} \mathrm{C}$}

half year

in -vapor in - water at - waterline in - vapor in - water at - waterline

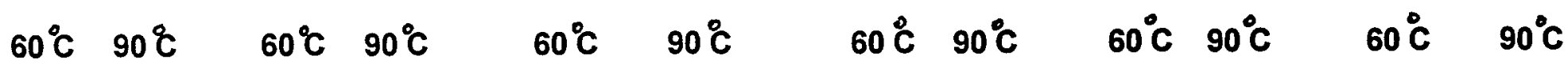

$\begin{array}{lllllllllllll}\text { Monel } 400 & 1 & 4 & 118 & 92 & 87 & 59 & 1 & 2 & 61 & 52 & 54 & 38\end{array}$

CDA 715

$\begin{array}{llllll}4 & 11 & 219 & 282 & 403 & 285\end{array}$

39

117215

216

221 


\section{Table 3 : Corrosion rate (micron/year) of corrosion resistant materials after half year in vapor above and in SAW solution at $60^{\circ} \mathrm{C} \& 90^{\circ} \mathrm{C}$}

temp, ${ }^{\circ} c \quad$ in vapor in water

Alloy 825

$\begin{array}{lll}60 & 0.03 & 0.58 \\ 90 & 0.08 & 1.33\end{array}$

Alloy G3

$\begin{array}{lll}60 & 0.02 & 0.01 \\ 90 & 0.04 & 0.12\end{array}$

90

Alloy C22

$\begin{array}{lll}60 & 0.06 & 0.04 \\ 90 & 0.06 & 0.1\end{array}$

Alloy C4

$\begin{array}{lll}60 & 0.06 & 0.08 \\ 90 & 0.06 & 0.13\end{array}$

temp ${ }^{\circ} \mathrm{C}$ in vapor in water

$\begin{array}{llll}\text { Titanium Gr } 12 & 60 & 7.97 & 4.48\end{array}$

$\begin{array}{lll}90 & 7.78 & 3.96\end{array}$

$\begin{array}{llll}\text { Titanium Gr } 16 & 60 & 5.49 & 2.05\end{array}$

$90 \quad 4.43 \quad 2.3$

Inconel 625

$60 \quad 0.05 \quad-0.01$

$90 \quad 0.03 \quad-0.01$ 
Table 4 : Corrosion rate (micron/year) of Alloy C22 and Inconel 625 above and in SAW at $60^{\circ} \mathrm{C}$ after one year

in-vapor

C22

Inconel 625

$$
0.03
$$

0.01 in-water

0.01 


\section{Corrosion Rate Calculations for Weight Loss Specimens}

For Rack Number : 23-1

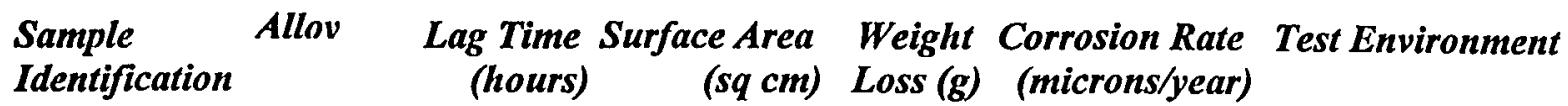

\begin{tabular}{|c|c|c|c|c|c|c|}
\hline IWA 001 & $\begin{array}{l}\text { A387 } \\
\text { Grade } 22\end{array}$ & 4536 & 28.680 & 0.2073 & 1776 & $60 \mathrm{C}$ SDW VAPOR PHASE \\
\hline IWA 002 & $\begin{array}{l}\text { A387 } \\
\text { Grade } 22\end{array}$ & 4536 & 28.857 & 02647 & 22.54 & $60 \mathrm{C}$ SDW VAPOR PHASE \\
\hline IWA 003 & $\begin{array}{l}\text { A387 } \\
\text { Grade } 22\end{array}$ & 4536 & 29.182 & 0.5066 & 42.65 & $60 \mathrm{C}$ SDW VAPOR PHASE \\
\hline IWA 004 & $\begin{array}{l}\text { A387 } \\
\text { Grade } 22\end{array}$ & 4536 & 28.431 & 0.5857 & 50.62 & $60 \mathrm{C}$ SDW AQUEOUS PHASE \\
\hline IWA 005 & $\begin{array}{l}\text { A387 } \\
\text { Grade } 22\end{array}$ & 4536 & 29.017 & 0.2817 & 23.85 & $60 \mathrm{C}$ SDW AQUEOUS PHASE \\
\hline IWA 006 & $\begin{array}{l}\text { A387 } \\
\text { Grade } 22\end{array}$ & 4536 & 29.034 & 1.4452 & 122.30 & $60 \mathrm{C}$ SDW AQUEOUS PHASE \\
\hline IWA 031 & $\begin{array}{l}\text { A387 } \\
\text { Grade } 22\end{array}$ & 4536 & 29.005 & 1.6293 & 138.02 & $60 \mathrm{C}$ SDW WATERLINE \\
\hline IWH 001 & $\begin{array}{l}\text { A387 } \\
\text { Grade } 22\end{array}$ & 4536 & 29.122 & 0.2385 & 20.12 & $60 \mathrm{C}$ SDW VAPOR PHASE \\
\hline IWH 002 & $\begin{array}{l}\text { A387 } \\
\text { Grade } 22\end{array}$ & 4536 & 27.941 & 0.3676 & 32.33 & $60 \mathrm{C}$ SDW VAPOR PHASE \\
\hline IWH 003 & $\begin{array}{l}\text { A387 } \\
\text { Grade } 22\end{array}$ & 4536 & 29.366 & 0.6853 & 57.34 & $60 \mathrm{C}$ SDW VAPOR PHASE \\
\hline IWH 004 & $\begin{array}{l}\text { A387 } \\
\text { Grade } 22\end{array}$ & 4536 & 29.099 & 0.4067 & 34.34 & $60 \mathrm{C}$ SDW AQUEOUS PHASE \\
\hline IWH 005 & $\begin{array}{l}\text { A387 } \\
\text { Grade } 22\end{array}$ & 4536 & 29.045 & 0.3622 & 30.64 & $60 \mathrm{C}$ SDW AQUEOUS PHASE \\
\hline WH 006 & $\begin{array}{l}\text { A387 } \\
\text { Grade } 22\end{array}$ & 4536 & 29.178 & 0.4538 & 38.21 & $60 \mathrm{C}$ SDW AQUEOUS PHASE \\
\hline IWH 031 & $\begin{array}{l}\text { A387 } \\
\text { Grade } 22\end{array}$ & 4536 & 28.990 & 1.6319 & 138.31 & $60 \mathrm{C}$ SDW WATERLINE \\
\hline JWA 001 & $\begin{array}{l}\text { A516 } \\
\text { Grade } 55\end{array}$ & 4536 & 28.702 & 0.3233 & 27.68 & $60 \mathrm{C}$ SDW VAPOR PHASE \\
\hline JWA 002 & $\begin{array}{l}\text { A516 } \\
\text { Grade } 55\end{array}$ & 4536 & 28.859 & 0.2539 & 21.62 & 60 C SDW VAPOR PHASE \\
\hline JWA 003 & $\begin{array}{l}\text { A516 } \\
\text { Grade } 55\end{array}$ & 4536 & 28.658 & 0.4892 & 41.94 & $60 \mathrm{C}$ SDW VAPOR PHASE \\
\hline JWA 004 & $\begin{array}{l}\text { A516 } \\
\text { Grade } 55\end{array}$ & 4536 & 28.403 & 1.1677 & 101.01 & $60 \mathrm{C}$ SDW AQUEOUS PHASE \\
\hline WA 005 & $\begin{array}{l}\text { A516 } \\
\text { Grade } 55\end{array}$ & 4536 & 28.319 & 1.2497 & 108.43 & $60 \mathrm{C}$ SDW AQUEOUS PHASE \\
\hline
\end{tabular}




\section{Corrosion Rate Calculations for Weight Loss Specimens}

\section{For Rack Number : 23-1}

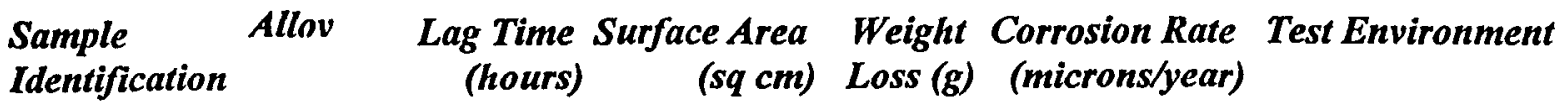

\begin{tabular}{|c|c|c|c|c|c|c|}
\hline JWA 006 & $\begin{array}{l}\text { A516 } \\
\text { Grade } 55\end{array}$ & 4536 & 28.806 & 1.2349 & 105.33 & $60 \mathrm{C}$ SDW AQUEOUS PHASE \\
\hline NWA 031 & $\begin{array}{l}\text { A516 } \\
\text { Grade } 55\end{array}$ & 4536 & 29.192 & 2.6075 & 219.46 & $60 \mathrm{C}$ SDW WATERLINE \\
\hline sWi 001 & $\begin{array}{l}\text { A516 } \\
\text { Grade } 55\end{array}$ & 4536 & 27.504 & 0.4384 & 39.16 & $60 \mathrm{C}$ SDW VAPOR PHASE \\
\hline JWI 002 & $\begin{array}{l}\text { A516 } \\
\text { Grade } 55\end{array}$ & 4536 & 27.764 & 0.4193 & 37.11 & $60 \mathrm{C}$ SDW VAPOR PHASE \\
\hline JWI 003 & $\begin{array}{l}\text { A516 } \\
\text { Grade } 55\end{array}$ & 4536 & 27.419 & 0.6981 & 62.56 & $60 \mathrm{C}$ SDW VAPOR PHASE \\
\hline SWI 004 & $\begin{array}{l}\text { A516 } \\
\text { Grade } 55\end{array}$ & 4536 & 28.094 & 1.0079 & 88.15 & $60 \mathrm{C}$ SDW AQUEOUS PHASE \\
\hline JWI 005 & $\begin{array}{l}\text { A516 } \\
\text { Grade } 55\end{array}$ & 4536 & 27.486 & 1.1405 & 101.95 & $60 \mathrm{C}$ SDW AQUEOUS PHASE \\
\hline JWI 006 & $\begin{array}{l}\text { A516 } \\
\text { Grade } 55\end{array}$ & 4536 & 27.887 & 1.3384 & 117.92 & $60 \mathrm{C}$ SDW AQUEOUS PHASE \\
\hline JWI 031 & $\begin{array}{l}\text { A516 } \\
\text { Grade } 55\end{array}$ & 4536 & 27.852 & 2.5852 & 228.06 & $60 \mathrm{C}$ SDW WATERLINE \\
\hline KWA 001 & $\begin{array}{l}\text { A27 Grade } \\
70-40\end{array}$ & 4536 & 28.344 & 0.4362 & 38.01 & $60 \mathrm{C}$ SDW VAPOR PHASE \\
\hline KWA 002 & $\begin{array}{l}\text { A27 Grade } \\
70-40\end{array}$ & 4536 & 28.170 & 0.2313 & 20.28 & $60 \mathrm{C}$ SDW VAPOR PHASE \\
\hline KWA 003 & $\begin{array}{l}\text { A27 Grade } \\
70-40\end{array}$ & 4536 & 28.230 & 0.4351 & 38.06 & $60 \mathrm{C}$ SDW VAPOR PHASE \\
\hline KWA 004 & $\begin{array}{l}\text { A27 Grade } \\
70-40\end{array}$ & 4536 & 28.060 & 1.3262 & 116.72 & $60 \mathrm{C}$ SDW AQUEOUS PHASE \\
\hline KWA 005 & $\begin{array}{l}\text { A27 Grade } \\
70-40\end{array}$ & 4536 & 28.095 & 0.4700 & 41.31 & $60 \mathrm{C}$ SDW AQUEOUS PHASE \\
\hline KWA 006 & $\begin{array}{l}\text { A27 Grade } \\
70-40\end{array}$ & 4536 & 28.154 & 1.0919 & 95.78 & $60 \mathrm{C}$ SDW AQUEOUS PHASE \\
\hline KWA 031 & $\begin{array}{l}\text { A27 Grade } \\
70-40\end{array}$ & 4536 & 28.220 & 2.3064 & 201.84 & $60 \mathrm{C}$ SDW WATERLINE \\
\hline KWI 001 & $\begin{array}{l}\text { A27 Grade } \\
70-40\end{array}$ & 4536 & 28.537 & 0.7067 & 61.16 & $60 \mathrm{C}$ SDW VAPOR PHASE \\
\hline KWI 002 & $\begin{array}{l}\text { A27 Grade } \\
70-40\end{array}$ & 4536 & 28.451 & 0.5307 & 46.07 & $60 \mathrm{C}$ SDW VAPOR PHASE \\
\hline KWI 003 & $\begin{array}{l}\text { A27 Grade } \\
70-40\end{array}$ & 4536 & 28.088 & 0.8026 & 70.57 & 60 C SDW VAPOR PHASE \\
\hline
\end{tabular}




\section{Corrosion Rate Calculations for Weight Loss Specimens}

For Rack Number : 23-1

\begin{tabular}{|c|c|c|c|c|c|c|}
\hline $\begin{array}{l}\text { Sample } \\
\text { Identification }\end{array}$ & Allov & $\begin{array}{r}\text { Lag Time } \\
\text { (hours) }\end{array}$ & $\begin{array}{r}\text { Surface Area } \\
(\mathrm{sq} \mathrm{cm})\end{array}$ & $\begin{array}{l}\text { Weight } \\
\text { Loss }(g)\end{array}$ & $\begin{array}{l}\text { Corrosion Rate } \\
\text { (microns/year) }\end{array}$ & Test Environment \\
\hline KWI 004 & $\begin{array}{l}\text { A27 Grade } \\
70-40\end{array}$ & 4536 & 28.264 & 1.1524 & 100.69 & $60 \mathrm{C}$ SDW AQUEOUS PHASE \\
\hline KWI 005 & $\begin{array}{l}\text { A27 Grade } \\
70-40\end{array}$ & 4536 & 27.888 & 1.0008 & 88.62 & $60 \mathrm{C}$ SDW AQUEOUS PHASE \\
\hline KWI 006 & $\begin{array}{l}\text { A27 Grade } \\
70-40\end{array}$ & 4536 & 28.533 & 1.2701 & 109.93 & $60 \mathrm{C}$ SDW AQUEOUS PHASE \\
\hline KWI 031 & $\begin{array}{l}\text { A27 Grade } \\
70-40\end{array}$ & 4536 & 27.372 & 2.2139 & 199.75 & $60 \mathrm{C}$ SDW WATERLINE \\
\hline
\end{tabular}




\section{Corrosion Rate Calculations for Weight Loss Specimens}

For Rack Number : 23-2

Sample Allov Lag Time Surface Area Weight Corrosion Rate Test Environment Identification (hours) (sq cm) Loss (g) (microns/year)

\begin{tabular}{|c|c|c|c|c|c|c|}
\hline IWA 007 & $\begin{array}{l}\text { A387 } \\
\text { Grade } 22\end{array}$ & 8760 & 29.049 & 0.2910 & 12.74 & $60 \mathrm{C}$ SDW VAPOR PHASE \\
\hline IWA 008 & $\begin{array}{l}\text { A387 } \\
\text { Grade } 22\end{array}$ & 8760 & 28.523 & 0.3327 & 14.84 & $60 \mathrm{C}$ SDW VAPOR PHASE \\
\hline IWA 009 & $\begin{array}{l}\text { A387 } \\
\text { Grade } 22\end{array}$ & 8760 & 28.945 & 0.6259 & 27.51 & $60 \mathrm{C}$ SDW VAPOR PHASE \\
\hline WA 010 & $\begin{array}{l}\text { A387 } \\
\text { Grade } 22\end{array}$ & 8760 & 28.539 & 2.0178 & 89.95 & $60 \mathrm{C}$ SDW AQUEOUS PHASE \\
\hline IWA 011 & $\begin{array}{l}\text { A387 } \\
\text { Grade } 22\end{array}$ & 8760 & 28.297 & 2.2737 & 102.23 & $60 \mathrm{C}$ SDW AQUEOUS PHASE \\
\hline WA 012 & $\begin{array}{l}\text { A387 } \\
\text { Grade } 22\end{array}$ & 8760 & 28.950 & 2.7486 & 120.79 & $60 \mathrm{C}$ SDW AQUEOUS PHASE \\
\hline IWA 032 & $\begin{array}{l}\text { A387 } \\
\text { Grade } 22\end{array}$ & 8760 & 28.965 & 3.6390 & 159.84 & $60 \mathrm{C}$ SDW WATERLINE \\
\hline MH 007 & $\begin{array}{l}\text { A387 } \\
\text { Grade } 22\end{array}$ & 8760 & 29.240 & 0.4200 & 18.27 & $60 \mathrm{C}$ SDW VAPOR PHASE \\
\hline WH 008 & $\begin{array}{l}\text { A387 } \\
\text { Grade } 22\end{array}$ & 8760 & 29.149 & 0.5590 & 24.40 & $60 \mathrm{C}$ SDW VAPOR PHASE \\
\hline WH 009 & $\begin{array}{l}\text { A387 } \\
\text { Grade } 22\end{array}$ & 8760 & 28.941 & 0.8521 & 37.46 & $60 \mathrm{C}$ SDW VAPOR PHASE \\
\hline WH 010 & $\begin{array}{l}\text { A387 } \\
\text { Grade } 22\end{array}$ & 8760 & 29.111 & 2.6131 & 114.20 & $60 \mathrm{C}$ SDW AQUEOUS PHASE \\
\hline IWH 011 & $\begin{array}{l}\text { A387 } \\
\text { Grade } 22\end{array}$ & 8760 & 28.781 & 2.4676 & 109.08 & $60 \mathrm{C}$ SDW AQUEOUS PHASE \\
\hline WH 012 & $\begin{array}{l}\text { A387 } \\
\text { Grade } 22\end{array}$ & 8760 & 29.173 & 0.4248 & 18.53 & $60 \mathrm{C}$ SDW AQUEOUS PHASE \\
\hline MH 032 & $\begin{array}{l}\text { A387 } \\
\text { Grade } 22\end{array}$ & 8760 & 29.276 & 3.5821 & 155.67 & $60 \mathrm{C}$ SDW WATERLINE \\
\hline JWA 007 & $\begin{array}{l}\text { A516 } \\
\text { Grade } 55\end{array}$ & 8760 & 28.702 & 0.4950 & 21.94 & $60 \mathrm{C}$ SDW VAPOR PHASE \\
\hline WA 008 & $\begin{array}{l}\text { A516 } \\
\text { Grade } 55\end{array}$ & 8760 & 28.494 & 0.5526 & 24.67 & $60 \mathrm{C}$ SDW VAPOR PHASE \\
\hline WA 009 & $\begin{array}{l}\text { A516 } \\
\text { Grade } 55\end{array}$ & 8760 & 28.134 & 0.8404 & 38.00 & $60 \mathrm{C}$ SDW VAPOR PHASE \\
\hline JWA 010 & $\begin{array}{l}\text { A516 } \\
\text { Grade } 55\end{array}$ & 8760 & 28.788 & 1.8102 & 80.00 & $60 \mathrm{C}$ SDW AQUEOUS PHASE \\
\hline WWA 011 & $\begin{array}{l}\text { A516 } \\
\text { Grade } 55\end{array}$ & 8760 & 28.663 & 1.5038 & 66.75 & $60 \mathrm{C}$ SDW AQUEOUS PHASE \\
\hline
\end{tabular}




\section{Corrosion Rate Calculations for Weight Loss Specimens}

\section{For Rack Number : 23-2}

Sample Allov Lag Time Surface Area Weight Corrosion Rate Test Environment Identification (hours) (sq cm) Loss (g) (microns/year)

\begin{tabular}{|c|c|c|c|c|c|c|}
\hline JWA 012 & $\begin{array}{l}\text { A516 } \\
\text { Grade } 55\end{array}$ & 8760 & 28.605 & 1.7383 & 77.31 & $60 \mathrm{C}$ SDW AQUEOUS PHASE \\
\hline JWA 032 & $\begin{array}{l}\text { A516 } \\
\text { Grade } 55\end{array}$ & 8760 & 28.386 & 3.7620 & 168.61 & $60 \mathrm{C}$ SDW WATERLINE \\
\hline JW1 007 & $\begin{array}{l}\text { A516 } \\
\text { Grade } 55\end{array}$ & 8760 & 27.840 & 0.4071 & 18.60 & $60 \mathrm{C}$ SDW VAPOR PHASE \\
\hline JWI 008 & $\begin{array}{l}\text { A516 } \\
\text { Grade } 55\end{array}$ & 8760 & 27.636 & 0.4418 & 20.34 & $60 \mathrm{C}$ SDW VAPOR PHASE \\
\hline JWI 009 & $\begin{array}{l}\text { A516 } \\
\text { Grade } 55\end{array}$ & 8760 & 27.516 & 0.8367 & 38.69 & $60 \mathrm{C}$ SDW VAPOR PHASE \\
\hline SWI 010 & $\begin{array}{l}\text { A516 } \\
\text { Grade } 55\end{array}$ & 8760 & 27.841 & 1.5283 & 69.84 & $60 \mathrm{C}$ SDW AQUEOUS PHASE \\
\hline JWI 011 & $\begin{array}{l}\text { A516 } \\
\text { Grade } 55\end{array}$ & 8760 & 27.684 & 1.8222 & 83.74 & $60 \mathrm{C}$ SDW AQUEOUS PHASE \\
\hline JWI 012 & $\begin{array}{l}\text { A516 } \\
\text { Grade } 55\end{array}$ & 8760 & 27.473 & 1.7121 & 79.29 & $60 \mathrm{C}$ SDW AQUEOUS PHASE \\
\hline JWI 032 & $\begin{array}{l}\text { A516 } \\
\text { Grade } 55\end{array}$ & 8760 & 28.022 & 4.8260 & 219.11 & $60 \mathrm{C}$ SDW WATERLINE \\
\hline KWA 007 & $\begin{array}{l}\text { A27 Grade } \\
70-40\end{array}$ & 8760 & 28.167 & 07855 & 35.66 & $60 \mathrm{C}$ SDW VAPOR PHASE \\
\hline KWA 008 & $\begin{array}{l}\text { A27 Grade } \\
70-40\end{array}$ & 8760 & 28.160 & 0.5552 & 25.21 & $60 \mathrm{C}$ SDW VAPOR PHASE \\
\hline KWA 009 & $\begin{array}{l}\text { A27 Grade } \\
70-40\end{array}$ & 8760 & 28.145 & 0.7363 & 33.45 & $60 \mathrm{C}$ SDW VAPOR PHASE \\
\hline KWA 010 & $\begin{array}{l}\text { A27 Grade } \\
70-40\end{array}$ & 8760 & 28.000 & 1.9919 & 90.97 & $60 \mathrm{C}$ SDW AQUEOUS PHASE \\
\hline KWA 011 & $\begin{array}{l}\text { A27 Grade } \\
70-40\end{array}$ & 8760 & 28.116 & 1.8306 & 83.26 & $60 \mathrm{C}$ SDW AQUEOUS PHASE \\
\hline KWA 012 & $\begin{array}{l}\text { A27 Grade } \\
70-40\end{array}$ & 8760 & 28.208 & 1.6387 & 74.29 & $60 \mathrm{C}$ SDW AQUEOUS PHASE \\
\hline KWA 032 & $\begin{array}{l}\text { A27 Grade } \\
70-40\end{array}$ & 8760 & 28.261 & 5.1990 & 235.24 & $60 \mathrm{C}$ SDW WATERLINE \\
\hline KWI 007 & $\begin{array}{l}\text { A27 Grade } \\
70-40\end{array}$ & 8760 & 27.807 & 0.7467 & 34.34 & $60 \mathrm{C}$ SDW VAPOR PHASE \\
\hline KWI 008 & $\begin{array}{l}\text { A27 Grade } \\
70-40\end{array}$ & 8760 & 28.043 & 0.8135 & 37.10 & $60 \mathrm{C}$ SDW VAPOR PHASE \\
\hline KWI 009 & $\begin{array}{l}\text { A27 Grade } \\
70-40\end{array}$ & 8760 & 28.058 & 0.9692 & 44.17 & $60 \mathrm{C}$ SDW VAPOR PHASE \\
\hline
\end{tabular}




\section{Corrosion Rate Calculations for Weight Loss Specimens}

For Rack Number : 23-2

Sample Allov Lag Time Surface Area Weight Corrosion Rate Test Environment

Identification (hours) (sq cm) Loss (g) (microns/year)

\begin{tabular}{lllllll}
\hline KWI 010 & $\begin{array}{l}\text { A27 Grade } \\
70-40\end{array}$ & 8760 & 28.517 & 1.6251 & 72.87 & 60 C SDW AQUEOUS PHASE \\
KWI 011 & $\begin{array}{l}\text { A27 Grade } \\
70-40\end{array}$ & 8760 & 28.387 & 1.6309 & 73.47 & 60 C SDW AQUEOUS PHASE \\
KWI 012 & $\begin{array}{l}\text { A27 Grade } \\
70-40\end{array}$ & 8760 & 27.954 & 1.8289 & 83.66 & 60 C SDW AQUEOUS PHASE \\
KWI 032 & $\begin{array}{l}\text { A27 Grade } \\
70-40\end{array}$ & 8760 & 28.037 & 3.9510 & 180.21 & 60 C SDW WATERLINE
\end{tabular}




\title{
Corrosion Rate Calculations for Weight Loss Specimens
} For Rack Number : 22-1

\author{
Sample Allov Lag Time Surface Area Weight Corrosion Rate Test Environment \\ Identification (hours) (sq cm) Loss (g) (microns/year)
}

\begin{tabular}{|c|c|c|c|c|c|c|}
\hline IWA 036 & $\begin{array}{l}\text { A387 } \\
\text { Grade } 22\end{array}$ & 4632 & 28.865 & 2.4882 & 207.41 & $90 \mathrm{C}$ SDW WATERLINE \\
\hline IWA 041 & $\begin{array}{l}\text { A387 } \\
\text { Grade } 22\end{array}$ & 4632 & 28.860 & 0.5304 & 44.22 & $90 \mathrm{C}$ SDW VAPOR PHASE \\
\hline IWA 042 & $\begin{array}{l}\text { A387 } \\
\text { Grade } 22\end{array}$ & 4632 & 28.879 & 0.5403 & 45.02 & $90 \mathrm{C}$ SDW VAPOR PHASE \\
\hline WA 043 & $\begin{array}{l}\text { A387 } \\
\text { Grade } 22\end{array}$ & 4632 & 28.935 & 0.4968 & 41.31 & 90 C SDW VAPOR PHASE \\
\hline IWA 044 & $\begin{array}{l}\text { A387 } \\
\text { Grade } 22\end{array}$ & 4632 & 28.799 & 1.3652 & 114.06 & $90 \mathrm{C}$ SDW AQUEOUS PHASE \\
\hline WA 045 & $\begin{array}{l}\text { A387 } \\
\text { Grade } 22\end{array}$ & 4632 & 28.691 & 1.2959 & 108.68 & $90 \mathrm{C}$ SDW AQUEOUS PHASE \\
\hline IWA 046 & $\begin{array}{l}\text { A387 } \\
\text { Grade } 22\end{array}$ & 4632 & 28.789 & 0.9445 & 78.94 & $90 \mathrm{C}$ SDW AQUEOUS PHASE \\
\hline WH 036 & $\begin{array}{l}\text { A387 } \\
\text { Grade } 22\end{array}$ & 4632 & 28.043 & 2.5592 & 219.58 & $90 \mathrm{C}$ SDW WATERLINE \\
\hline WH 041 & $\begin{array}{l}\text { A387 } \\
\text { Grade } 22\end{array}$ & 4632 & 29.113 & 0.6336 & 52.37 & $90 \mathrm{C}$ SDW VAPOR PHASE \\
\hline IWH 042 & $\begin{array}{l}\text { A387 } \\
\text { Grade } 22\end{array}$ & 4632 & 29.228 & 0.6235 & 51.33 & $90 \mathrm{C}$ SDW VAPOR PHASE \\
\hline WH 043 & $\begin{array}{l}\text { A387 } \\
\text { Grade } 22\end{array}$ & 4632 & 29.159 & 0.6283 & 51.85 & $90 \mathrm{C}$ SDW VAPOR PHASE \\
\hline WW 044 & $\begin{array}{l}\text { A387 } \\
\text { Grade } 22\end{array}$ & 4632 & 29.206 & 1.3804 & 113.72 & $90 \mathrm{C}$ SDW AQUEOUS PHASE \\
\hline MH 045 & $\begin{array}{l}\text { A387 } \\
\text { Grade } 22\end{array}$ & 4632 & 29.181 & 1.2912 & 106.46 & $90 \mathrm{C}$ SDW AQUEOUS PHASE \\
\hline MH 046 & $\begin{array}{l}\text { A387 } \\
\text { Grade } 22\end{array}$ & 4632 & 29.061 & 1.5030 & 124.44 & 90 C SDW AQUEOUS PHASE \\
\hline JWA 036 & $\begin{array}{l}\text { A516 } \\
\text { Grade } 55\end{array}$ & 4632 & 27.775 & 2.4751 & 214.42 & 90 C SDW WATERLINE \\
\hline JWA 041 & $\begin{array}{l}\text { A516 } \\
\text { Grade } 55\end{array}$ & 4632 & 28.835 & 0.8489 & 70.83 & $90 \mathrm{C}$ SDW VAPORPHASE \\
\hline JWA 042 & $\begin{array}{l}\text { A516 } \\
\text { Grade } 55\end{array}$ & 4632 & 28.573 & 0.7100 & 59.79 & $90 \mathrm{C}$ SDW VAPOR PHASE \\
\hline JWA 043 & $\begin{array}{l}\text { A516 } \\
\text { Grade } 55\end{array}$ & 4632 & 28.359 & 0.7533 & 63.91 & $90 \mathrm{C}$ SDW VAPOR PHASE \\
\hline NWA 044 & $\begin{array}{l}\text { A516 } \\
\text { Grade } 55\end{array}$ & 4632 & 29.056 & 1.0797 & 89.41 & $90 \mathrm{C}$ SDW AQUEOUS PHASE \\
\hline
\end{tabular}




\section{Corrosion Rate Calculations for Weight Loss Specimens}

\section{For Rack Number : 22-1}

Sample Allov Lag Time Surface Area Weight Corrosion Rate Test Environment Identification (hours) (sq cm) Loss (g) (microns/year)

\begin{tabular}{|c|c|c|c|c|c|c|}
\hline JWA 045 & $\begin{array}{l}\text { A516 } \\
\text { Grade } 55\end{array}$ & 4632 & 28.401 & 0.8133 & 68.90 & $90 \mathrm{C}$ SDW AQUEOUS PHASE \\
\hline JWA 046 & $\begin{array}{l}\text { A516 } \\
\text { Grade } 55\end{array}$ & 4632 & 29.481 & 1.0294 & 84.02 & $90 \mathrm{C}$ SDW AQUEOUS PHASE \\
\hline SWI 036 & $\begin{array}{l}\text { A516 } \\
\text { Grade } 55\end{array}$ & 4632 & 28.050 & 3.1770 & 272.52 & SOC SDW WATERLINE \\
\hline JWI 041 & $\begin{array}{l}\text { A516 } \\
\text { Grade } 55\end{array}$ & 4632 & 27.897 & 0.8859 & 76.41 & $90 \mathrm{C}$ SDW VAPOR PHASE \\
\hline JW1 042 & $\begin{array}{l}\text { A516 } \\
\text { Grade } 55\end{array}$ & 4632 & 27.940 & 1.0990 & 94.64 & $90 \mathrm{C}$ SDW VAPOR PHASE \\
\hline JWI 043 & $\begin{array}{l}\text { A516 } \\
\text { Grade } 55\end{array}$ & 4632 & 28.116 & 1.2261 & 104.93 & $90 \mathrm{C}$ SDW VAPOR PHASE \\
\hline JWI 044 & $\begin{array}{l}\text { A516 } \\
\text { Grade } 55\end{array}$ & 4632 & 28.120 & 1.0243 & 87.65 & $90 \mathrm{C}$ SDW AQUEOUS PHASE \\
\hline JWI 045 & $\begin{array}{l}\text { A516 } \\
\text { Grade } 55\end{array}$ & 4632 & 27.657 & 1.2352 & 107.46 & $90 \mathrm{C}$ SDW AQUEOUS PHASE \\
\hline JW1 046 & $\begin{array}{l}\text { A516 } \\
\text { Grade } 55\end{array}$ & 4632 & 28.402 & 1.5348 & 130.02 & $90 \mathrm{C}$ SDW AQUEOUS PHASE \\
\hline KWA 036 & $\begin{array}{l}\text { A27 Grade } \\
70-40\end{array}$ & 4632 & 28.150 & 3.1445 & 270.15 & $90 \mathrm{C}$ SDW WATERLINE \\
\hline KWA 041 & $\begin{array}{l}\text { A27 Grade } \\
70-40\end{array}$ & 4632 & 28.158 & 1.0636 & 91.35 & $90 \mathrm{C}$ SDW VAPOR PHASE \\
\hline KWA 042 & $\begin{array}{l}\text { A27 Grade } \\
70-40\end{array}$ & 4632 & 28.285 & 0.5573 & 47.65 & $90 \mathrm{C}$ SDW VAPOR PHASE \\
\hline KWA 043 & $\begin{array}{l}\text { A27 Grade } \\
70-40\end{array}$ & 4632 & 27.836 & 0.7197 & 62.53 & $90 \mathrm{C}$ SDW VAPOR PHASE \\
\hline KWA 044 & $\begin{array}{l}\text { A27 Grade } \\
70-40\end{array}$ & 4632 & 28.305 & 0.8423 & 71.97 & $90 \mathrm{C}$ SDW AQUEOUS PHASE \\
\hline KWA 045 & $\begin{array}{l}\text { A27 Grade } \\
70-40\end{array}$ & 4632 & 28.192 & 0.9280 & 79.61 & $90 \mathrm{C}$ SDW AQUEOUS PHASE \\
\hline KWA 046 & $\begin{array}{l}\text { A27 Grade } \\
70-40\end{array}$ & 4632 & 28.021 & 1.1289 & $\mathbf{9 7 . 4 3}$ & $90 \mathrm{C}$ SDW AQUEOUS PHASE \\
\hline KW1 036 & $\begin{array}{l}\text { A27 Grade } \\
70-40\end{array}$ & 4632 & 28.260 & 2.4648 & 210.93 & $90 \mathrm{C}$ SDW WATERLINE \\
\hline KWI 041 & $\begin{array}{l}\text { A27 Grade } \\
70-40\end{array}$ & 4632 & 27.655 & 1.1642 & 101.81 & $90 \mathrm{C}$ SDW VAPOR PHASE \\
\hline KWI 042 & $\begin{array}{l}\text { A27 Grade } \\
70-40\end{array}$ & 4632 & 28.215 & 0.7152 & 61.30 & $90 \mathrm{C}$ SDW VAPOR PHASE \\
\hline
\end{tabular}




\section{Corrosion Rate Calculations for Weight Loss Specimens}

For Rack Number : 22-1

\begin{tabular}{llccccc}
$\begin{array}{l}\text { Sample } \\
\text { Identification }\end{array}$ & Allov & $\begin{array}{c}\text { Lag Time Surface Area } \\
\text { (hours) }\end{array}$ & $\begin{array}{l}\text { Weight } \\
\text { (sq cm) }\end{array}$ & $\begin{array}{c}\text { Corrosion Rate }(\mathrm{g}) \\
\text { (microns/year) }\end{array}$ & \\
\hline KW1 043 & $\begin{array}{l}\text { A27 Grade } \\
70-40\end{array}$ & 4632 & 27.713 & 0.8577 & 74.85 & 90 C SDW VAPOR PHASE \\
KWI 044 & $\begin{array}{l}\text { A27 Grade } \\
70-40\end{array}$ & 4632 & 28.025 & 0.8749 & 75.50 & 90 C SDW AQUEOUS PHASE \\
KW1 045 & $\begin{array}{l}\text { A27 Grade } \\
70-40\end{array}$ & 4632 & 28.159 & 0.7917 & 67.99 & 90 C SDW AQUEOUS PHASE \\
KW1 046 & $\begin{array}{l}\text { A27 Grade } \\
70-40\end{array}$ & 4632 & 28.141 & 1.0459 & 89.88 & 90 C SDW AQUEOUS PHASE
\end{tabular}




\section{Corrosion Rate Calculations for Weight Loss Specimens}

For Rack Number : 22-2

Sample Allov Lag Time Surface Area Weight Corrosion Rate Test Environment Identification (hours) (sq cm) Loss (g) (microns/year)

\begin{tabular}{|c|c|c|c|c|c|c|}
\hline IWA 037 & $\begin{array}{l}\text { A387 } \\
\text { Grade } 22\end{array}$ & 8832 & 28.966 & 3.8451 & 167.51 & 90 C SDW WATERLINE \\
\hline IWA 047 & $\begin{array}{l}\text { A387 } \\
\text { Grade } 22\end{array}$ & 8832 & 28.815 & 0.8075 & 3536 & $90 \mathrm{C}$ SDW VAPOR PHASE \\
\hline IWA 048 & $\begin{array}{l}\text { A387 } \\
\text { Grade } 22\end{array}$ & 8832 & 28.958 & 0.8414 & 36.67 & $90 \mathrm{C}$ SDW VAPOR PHASE \\
\hline IWA 049 & $\begin{array}{l}\text { A387 } \\
\text { Grade } 22\end{array}$ & 8832 & 29.078 & 0.9785 & 42.46 & $90 \mathrm{C}$ SDW VAPOR PHASE \\
\hline IWA 050 & $\begin{array}{l}\text { A387 } \\
\text { Grade } 22\end{array}$ & 8832 & 28.917 & 1.7311 & 75.54 & $90 \mathrm{C}$ SDW AQUEOUS PHASE \\
\hline IWA 051 & $\begin{array}{l}\text { A387 } \\
\text { Grade } 22\end{array}$ & 8832 & 28.964 & 1.8040 & 78.59 & $90 \mathrm{C}$ SDW AQUEOUS PHASE \\
\hline IWA 052 & $\begin{array}{l}\text { A387 } \\
\text { Grade } 22\end{array}$ & 8832 & 28.873 & 1.8432 & 80.56 & $90 \mathrm{C}$ SDW AQUEOUS PHASE \\
\hline IWH 037 & $\begin{array}{l}\text { A387 } \\
\text { Grade } 22\end{array}$ & 8832 & 28.856 & 3.7071 & 162.12 & 90 C SDW WATERLINE \\
\hline IWH 047 & $\begin{array}{l}\text { A387 } \\
\text { Grade } 22\end{array}$ & 8832 & 29.004 & 1.1770 & 51.21 & $90 \mathrm{C}$ SDW VAPOR PHASE \\
\hline WH 048 & $\begin{array}{l}\text { A387 } \\
\text { Grade } 22\end{array}$ & 8832 & 29.043 & 0.9553 & 41.51 & 90C SDW VAPOR PHASE \\
\hline IWH 049 & $\begin{array}{l}\text { A387 } \\
\text { Grade } 22\end{array}$ & 8832 & 28.427 & 0.9245 & 41.04 & $90 \mathrm{C}$ SDW VAPOR PHASE \\
\hline IWH 050 & $\begin{array}{l}\text { A387 } \\
\text { Grade } 22\end{array}$ & 8832 & 28.965 & 1.7169 & 74.80 & $90 \mathrm{C}$ SDW AQUEOUS PHASE \\
\hline IWH 051 & $\begin{array}{l}\text { A387 } \\
\text { Grade } 22\end{array}$ & 8832 & 29.072 & 2.0195 & 87.66 & 90 C SDW AQUEOUS PHASE \\
\hline IWH 052 & $\begin{array}{l}\text { A387 } \\
\text { Grade } 22\end{array}$ & 8832 & 29.174 & 1.6894 & 73.07 & $90 \mathrm{C}$ SDW AQUEOUS PHASE \\
\hline SWA 037 & $\begin{array}{l}\text { A516 } \\
\text { Grade } 55\end{array}$ & 8832 & 28.503 & 1.9016 & 84.19 & 90 C SDW WATERLINE \\
\hline SWA 047 & $\begin{array}{l}\text { A516 } \\
\text { Grade } 55\end{array}$ & 8832 & 27.714 & 1.0232 & 46.59 & $90 \mathrm{C}$ SDW VAPOR PHASE \\
\hline JWA 048 & $\begin{array}{l}\text { A516 } \\
\text { Grade } 55\end{array}$ & 8832 & 29.186 & 1.0397 & 44.95 & $90 \mathrm{C}$ SDW VAPOR PHASE \\
\hline JWA 049 & $\begin{array}{l}\text { A516 } \\
\text { Grade } 55\end{array}$ & 8832 & 29.058 & 1.0305 & 44.75 & $90 \mathrm{C}$ SDW VAPOR PHASE \\
\hline NWA 050 & $\begin{array}{l}\text { A516 } \\
\text { Grade } 55\end{array}$ & 8832 & 28.860 & 0.9982 & 43.65 & $90 \mathrm{C}$ SDW AQUEOUS PHASE \\
\hline
\end{tabular}




\section{Corrosion Rate Calculations for Weight Loss Specimens}

For Rack Number : 22-2

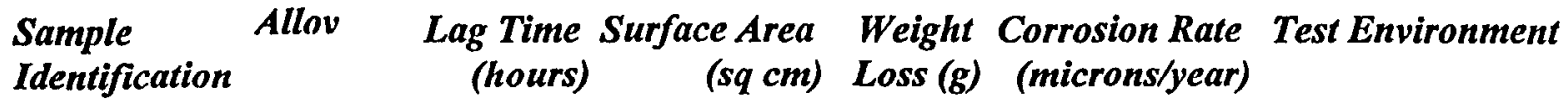

\begin{tabular}{|c|c|c|c|c|c|c|}
\hline JWA 051 & $\begin{array}{l}\text { A516 } \\
\text { Grade } 55\end{array}$ & 8832 & 28.722 & 1.0286 & 45.19 & $90 \mathrm{C}$ SDW AQUEOUS PHASE \\
\hline JWA 052 & $\begin{array}{l}\text { A516 } \\
\text { Grade } 55\end{array}$ & 8832 & 28.311 & 1.0836 & 48.30 & $90 \mathrm{C}$ SDW AQUEOUS PHASE \\
\hline SWI 037 & $\begin{array}{l}\text { A516 } \\
\text { Grade } 55\end{array}$ & 8832 & 27.796 & 3.0561 & 13874 & $90 \mathrm{C}$ SDW WATERLINE \\
\hline JWI 047 & $\begin{array}{l}\text { A516 } \\
\text { Grade } 55\end{array}$ & 8832 & 28.086 & 1.1352 & 51.00 & $90 \mathrm{C}$ SDW VAPOR PHASE \\
\hline JWI 048 & $\begin{array}{l}\text { A516 } \\
\text { Grade } 55\end{array}$ & 8832 & 28.264 & 1.3274 & 59.26 & $90 \mathrm{C}$ SDW VAPOR PHASE \\
\hline JWI 049 & $\begin{array}{l}\text { A516 } \\
\text { Grade } 55\end{array}$ & 8832 & 28021 & 1.3047 & 58.76 & $90 \mathrm{C}$ SDW VAPOR PHASE \\
\hline JWI 050 & $\begin{array}{l}\text { A516 } \\
\text { Grade } 55\end{array}$ & 8832 & 28.120 & 1.2473 & 55.97 & $90 \mathrm{C}$ SDW AQUEOUS PHASE \\
\hline JWI 051 & $\begin{array}{l}\text { A516 } \\
\text { Grade } 55\end{array}$ & 8832 & 28.252 & 1.4235 & 63.58 & 90 C SDW AQUEOUS PHASE \\
\hline SWI 052 & $\begin{array}{l}\text { A516 } \\
\text { Grade } 55\end{array}$ & 8832 & 28.285 & 1.1245 & 50.17 & $90 \mathrm{C}$ SDW AQUEOUS PHASE \\
\hline KWA 037 & $\begin{array}{l}\text { A27 Grade } \\
70-40\end{array}$ & 8832 & 28.143 & 2.3383 & 105.38 & $90 \mathrm{C}$ SDW WATERLINE \\
\hline KWA 047 & $\begin{array}{l}\text { A27 Grade } \\
70-40\end{array}$ & 8832 & 28.026 & 0.9801 & 44.36 & $90 \mathrm{C}$ SDW VAPOR PHASE \\
\hline KWA 048 & $\begin{array}{l}\text { A27 Grade } \\
70-40\end{array}$ & 8832 & 28.122 & 0.6153 & 27.75 & $90 \mathrm{C}$ SDW VAPOR PHASE \\
\hline KWA 049 & $\begin{array}{l}\text { A27 Grade } \\
70-40\end{array}$ & 8832 & 28.214 & 0.5048 & 22.69 & $90 \mathrm{C}$ SDW VAPOR PHASE \\
\hline KWA 050 & $\begin{array}{l}\text { A27 Grade } \\
70-40\end{array}$ & 8832 & 28.176 & 1.0395 & 46.79 & $90 \mathrm{C}$ SDW AQUEOUS PHASE \\
\hline KWA 051 & $\begin{array}{l}\text { A27 Grade } \\
70-40\end{array}$ & 8832 & 27.899 & 1.5213 & 68.91 & $90 \mathrm{C}$ SDW AQUEOUS PHASE \\
\hline KWA 052 & $\begin{array}{l}\text { A27 Grade } \\
70-40\end{array}$ & 8832 & 27.890 & 1.0273 & 46.72 & $90 \mathrm{C}$ SDW AQUEOUS PHASE \\
\hline KWI 037 & $\begin{array}{l}\text { A27 Grade } \\
70-40\end{array}$ & 8832 & 27.280 & 2.8428 & 132.17 & 9OC SDW WATERLINE \\
\hline KWI 047 & $\begin{array}{l}\text { A27 Grade } \\
70-40\end{array}$ & 8832 & 28.159 & 0.8939 & 40.26 & $90 \mathrm{C}$ SDW VAPOR PHASE \\
\hline KWI 048 & $\begin{array}{l}\text { A27 Grade } \\
70-40\end{array}$ & 8832 & 28.077 & 0.6022 & 27.20 & $90 \mathrm{C}$ SDW VAPOR PHASE \\
\hline
\end{tabular}




\section{Corrosion Rate Calculations for Weight Loss Specimens}

For Rack Number : 22-2

$\begin{array}{lcrl}\text { Sample Allov } & \text { Lag Time Surface Area Weight Corrosion Rate Test Environment } \\ \text { Identification } & \text { (hours) } & \text { (sq cm) Loss }(g) \text { (microns/year) }\end{array}$

\begin{tabular}{lllllll}
\hline KWI 049 & $\begin{array}{l}\text { A27 Grade } \\
70-40\end{array}$ & 8832 & 28.393 & 0.6925 & 30.94 & $90 \mathrm{C}$ SDW VAPOR PHASE \\
KW1 050 & $\begin{array}{l}\text { A27 Grade } \\
70-40\end{array}$ & 8832 & 28158 & 1.0281 & 46.31 & $90 \mathrm{C}$ SDW AQUEOUS PHASE \\
KW1 051 & $\begin{array}{l}\text { A27 Grade } \\
70-40\end{array}$ & 8832 & 28.396 & 0.8144 & 36.38 & $90 \mathrm{C}$ SDW AQUEOUS PHASE \\
KW1 052 & $\begin{array}{l}\text { A27 Grade } \\
70-40\end{array}$ & 8832 & 27.931 & 1.4508 & 65.88 & $90 \mathrm{C}$ SDW AQUEOUS PHASE
\end{tabular}




\section{Corrosion Rate Calculations for Weight Loss Specimens}

\section{For Rack Number : 21-1}

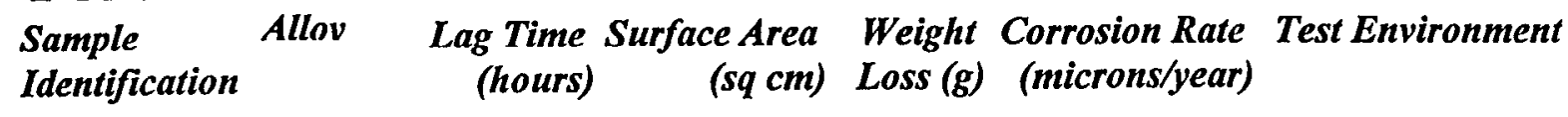

\begin{tabular}{|c|c|c|c|c|c|c|}
\hline IWA 071 & $\begin{array}{l}\text { A387 } \\
\text { Grade } 22\end{array}$ & 4392 & 27.260 & 0.9572 & 89.10 & $60 \mathrm{C}$ SCW VAPOR PHASE \\
\hline IWA 072 & $\begin{array}{l}\text { A387 } \\
\text { Grade } 22\end{array}$ & 4392 & 28.963 & 1.5336 & 134.36 & $60 \mathrm{C}$ SCW VAPOR PHASE \\
\hline WA 073 & $\begin{array}{l}\text { A387 } \\
\text { Grade } 22\end{array}$ & 4392 & 29.053 & 1.9986 & 174.56 & $60 \mathrm{C}$ SCW VAPOR PHASE \\
\hline IWA 074 & $\begin{array}{l}\text { A387 } \\
\text { Grade } 22\end{array}$ & 4392 & 29.069 & 0.2220 & 19.38 & $60 \mathrm{C}$ SCW AQUEOUS PHASE \\
\hline IWA 075 & $\begin{array}{l}\text { A387 } \\
\text { Grade } 22\end{array}$ & 4392 & 28.765 & 0.1779 & 15.69 & $60 \mathrm{C}$ SCW AQUEOUS PHASE \\
\hline MA 076 & $\begin{array}{l}\text { A387 } \\
\text { Grade } 22\end{array}$ & 4392 & 28.912 & 0.2982 & 26.17 & $60 \mathrm{C}$ SCW AQUEOUS PHASE \\
\hline IWA 101 & $\begin{array}{l}\text { A387 } \\
\text { Grade } 22\end{array}$ & 4392 & 28.805 & 1.8391 & 162.02 & $60 \mathrm{C}$ SCW WATERLINE \\
\hline IWH 071 & $\begin{array}{l}\text { A387 } \\
\text { Grade } 22\end{array}$ & 4392 & 29.209 & 1.2434 & 108.02 & $60 \mathrm{C}$ SCW VAPOR PHASE \\
\hline IWH 072 & $\begin{array}{l}\text { A387 } \\
\text { Grade } 22\end{array}$ & 4392 & 29.178 & 1.3570 & 118.02 & $60 \mathrm{C}$ SCW VAPOR PHASE \\
\hline MH 073 & $\begin{array}{l}\text { A387 } \\
\text { Grade } 22\end{array}$ & 4392 & 29.194 & 1.9149 & 166.45 & $60 \mathrm{C}$ SCW VAPOR PHASE \\
\hline IWH 074 & $\begin{array}{l}\text { A387 } \\
\text { Grade } 22\end{array}$ & 4392 & 29.121 & 0.3411 & 29.72 & $60 \mathrm{C}$ SCW AQUEOUS PHASE \\
\hline WH 075 & $\begin{array}{l}\text { A387 } \\
\text { Grade } 22\end{array}$ & 4392 & 29.260 & 0.3521 & 30.54 & $60 \mathrm{C}$ SCW AQUEOUS PHASE \\
\hline MH 076 & $\begin{array}{l}\text { A387 } \\
\text { Grade } 22\end{array}$ & 4392 & 29.173 & 0.3025 & 26.31 & $60 \mathrm{C}$ SCW AQUEOUS PHASE \\
\hline WWH 101 & $\begin{array}{l}\text { A387 } \\
\text { Grade } 22\end{array}$ & 4392 & 28.140 & 2.0023 & 174.36 & $60 \mathrm{C}$ SCW WATERLINE \\
\hline JWA 071 & $\begin{array}{l}\text { A516 } \\
\text { Grade } 55\end{array}$ & 4392 & 28.558 & 2.2380 & 198.86 & $60 \mathrm{C}$ SCW VAPOR PHASE \\
\hline SWA 072 & $\begin{array}{l}\text { A516 } \\
\text { Grade } 55\end{array}$ & 4392 & 27.265 & 2.8459 & 264.87 & $60 \mathrm{C}$ SCW VAPOR PHASE \\
\hline JWA 073 & $\begin{array}{l}\text { A516 } \\
\text { Grade } 55\end{array}$ & 4392 & 27.463 & 3.1926 & 295.00 & $60 \mathrm{C}$ SCW VAPOR PHASE \\
\hline JWA 074 & $\begin{array}{l}\text { A516 } \\
\text { Grade } 55\end{array}$ & 4392 & 28.585 & 0.6150 & 54.59 & $60 \mathrm{C}$ SCW AQUEOUS PHASE. \\
\hline JWA 075 & $\begin{array}{l}\text { A516 } \\
\text { Grade } 55\end{array}$ & 4392 & 28.711 & 0.6219 & 54.97 & $60 \mathrm{C}$ SCW AQUEOUS PHASE \\
\hline
\end{tabular}




\section{Corrosion Rate Calculations for Weight Loss Specimens}

For Rack Number : 21-1

Sample Identification

\begin{tabular}{|c|c|c|c|c|c|c|}
\hline JWA 076 & $\begin{array}{l}\text { A516 } \\
\text { Grade } 55\end{array}$ & 4392 & 28.415 & 0.5627 & 50.25 & $60 \mathrm{C}$ SCW AQUEOUS PHASE \\
\hline JWA 101 & $\begin{array}{l}\text { A516 } \\
\text { Grade } 55\end{array}$ & 4392 & 29.649 & 2.3122 & 197.90 & $60 \mathrm{C}$ SCW WATERLINE \\
\hline JWI 071 & $\begin{array}{l}\text { A516 } \\
\text { Grade } 55\end{array}$ & 4392 & 28.004 & 1.7925 & 162.43 & $60 \mathrm{C}$ SCW VAPOR PHASE \\
\hline JWI 072 & $\begin{array}{l}\text { A516 } \\
\text { Grade } 55\end{array}$ & 4392 & 28.018 & 1.7055 & 154.47 & $60 \mathrm{C}$ SCW VAPOR PHASE \\
\hline JWI 073 & $\begin{array}{l}\text { A516 } \\
\text { Grade } 55\end{array}$ & 4392 & 28.018 & 2.1305 & 192.96 & $60 \mathrm{C}$ SCW VAPOR PHASE \\
\hline JWI 074 & $\begin{array}{l}\text { A516 } \\
\text { Grade } 55\end{array}$ & 4392 & 27.742 & 0.7498 & 68.58 & $60 \mathrm{C}$ SCW AQUEOUS PHASE \\
\hline WW 075 & $\begin{array}{l}\text { A516 } \\
\text { Grade } 55\end{array}$ & 4392 & 27.897 & 0.9368 & 85.21 & $60 \mathrm{C}$ SCW AQUEOUS PHASE \\
\hline JWI 076 & $\begin{array}{l}\text { A516 } \\
\text { Grade } 55\end{array}$ & 4392 & 28.100 & 1.0138 & 91.55 & $60 \mathrm{C}$ SCW AQUEOUS PHASE \\
\hline WI 101 & $\begin{array}{l}\text { A516 } \\
\text { Grade } 55\end{array}$ & 4392 & 27.862 & 2.5297 & 230.40 & $60 \mathrm{C}$ SCW WATERLINE \\
\hline KWA 071 & $\begin{array}{l}\text { A27 Grade } \\
70-40\end{array}$ & 4392 & 28.134 & 1.9195 & 174.02 & $60 \mathrm{C}$ SCW VAPOR PHASE \\
\hline KWA 072 & $\begin{array}{l}\text { A27 Grade } \\
70-40\end{array}$ & 4392 & 28.061 & 2.4878 & 226.13 & $60 \mathrm{C}$ SCW VAPOR PHASE \\
\hline KWA 073 & $\begin{array}{l}\text { A27 Grade } \\
70-40\end{array}$ & 4392 & 28.175 & 3.9854 & 360.78 & $60 \mathrm{C}$ SCW VAPOR PHASE \\
\hline KWA 074 & $\begin{array}{l}\text { A27 Grade } \\
70-40\end{array}$ & 4392 & 28.402 & 0.8134 & 73.05 & $60 \mathrm{C}$ SCW AQUEOUS PHASE \\
\hline KWA 075 & $\begin{array}{l}\text { A27 Grade } \\
70-40\end{array}$ & 4392 & 28.360 & 0.8716 & 78.39 & $60 \mathrm{C}$ SCW AQUEOUS PHASE \\
\hline KWA 076 & $\begin{array}{l}\text { A27 Grade } \\
70-40\end{array}$ & 4392 & 27.828 & 0.7729 & 70.84 & $60 \mathrm{C}$ SCW AQUEOUS PHASE \\
\hline KWA 101 & $\begin{array}{l}\text { A27 Grade } \\
70-40\end{array}$ & 4392 & 28.223 & 2.0872 & 188.62 & $60 \mathrm{C}$ SCW WATERLINE \\
\hline KWI 071 & $\begin{array}{l}\text { A27 Grade } \\
70-40\end{array}$ & 4392 & 28.302 & 1.9898 & 179.32 & $60 \mathrm{C}$ SCW VAPOR PHASE \\
\hline KWI 072 & $\begin{array}{l}\text { A27 Grade } \\
70-40\end{array}$ & 4392 & 27.656 & 2.2157 & 204.34 & $60 \mathrm{C}$ SCW VAPOR PHASE \\
\hline KWI 073 & $\begin{array}{l}\text { A27 Grade } \\
70-40\end{array}$ & 4392 & 28.161 & 2.7674 & 250.65 & $60 \mathrm{C}$ SCW VAPOR PHASE \\
\hline
\end{tabular}

Weight Corrosion Rate Test Environment

(sq cm) Loss (g) (microns/year) 


\section{Corrosion Rate Calculations for Weight Loss Specimens}

\section{For Rack Number : 21-1}

\begin{tabular}{llccccc}
$\begin{array}{l}\text { Sample } \\
\text { Identification }\end{array}$ & Allov & $\begin{array}{c}\text { Lag Time Surface Area } \\
\text { (hours) }\end{array}$ & $\begin{array}{l}\text { Weight } \\
\text { (sq cm) }\end{array}$ & $\begin{array}{c}\text { Corrosion Rate } \\
\text { (microns/year) }\end{array}$ & Test Environment \\
\hline KW1 074 & $\begin{array}{l}\text { A27 Grade } \\
70-40\end{array}$ & 4392 & 28.373 & 0.5969 & 53.66 & $60 \mathrm{C}$ SCW AQUEOUS PHASE \\
KW1 075 & $\begin{array}{l}\text { A27 Grade } \\
70-40\end{array}$ & 4392 & 27.705 & 0.6270 & 57.72 & $60 \mathrm{C}$ SCW AQUEOUS PHASE \\
KW1 076 & $\begin{array}{l}\text { A27 Grade } \\
70-40\end{array}$ & 4392 & 28.098 & 0.6101 & 55.38 & $60 \mathrm{C}$ SCW AQUEOUS PHASE \\
KWI 101 & $\begin{array}{l}\text { A27 Grade } \\
70-40\end{array}$ & 4392 & 27.434 & 2.5415 & 236.28 & $60 \mathrm{C}$ SCW WATERLINE
\end{tabular}




\section{Corrosion Rate Calculations for Weight Loss Specimens}

For Rack Number : 21-2

Sample Allov Lag Time Surface Area Weight Corrosion Rate Test Environment Identification (hours) (sq cm) Loss (g) (microns/year)

\begin{tabular}{|c|c|c|c|c|c|c|}
\hline IWA 077 & $\begin{array}{l}\text { A387 } \\
\text { Grade } 22\end{array}$ & 8760 & 29.060 & 1.5731 & 68.87 & $60 \mathrm{C}$ SCW VAPOR PHASE \\
\hline IWA 078 & $\begin{array}{l}\text { A387 } \\
\text { Grade } 22\end{array}$ & 8760 & 28965 & 3.1742 & 139.42 & $60 \mathrm{C}$ SCW VAPOR PHASE \\
\hline IWA 079 & $\begin{array}{l}\text { A387 } \\
\text { Grade } 22\end{array}$ & 8760 & 28.773 & 4.0826 & 180.52 & $60 \mathrm{C}$ SCW VAPOR PHASE \\
\hline IWA 080 & $\begin{array}{l}\text { A387 } \\
\text { Grade } 22\end{array}$ & 8760 & 28.754 & 0.1690 & 7.48 & $60 \mathrm{C}$ SCW AQUEOUS PHASE \\
\hline IWA 081 & $\begin{array}{l}\text { A387 } \\
\text { Grade } 22\end{array}$ & 8760 & 28.554 & 0.1672 & 7.45 & $60 \mathrm{C}$ SCW AQUEOUS PHASE \\
\hline MA 082 & $\begin{array}{l}\text { A387 } \\
\text { Grade } 22\end{array}$ & 8760 & 27.303 & 0.1270 & 5.92 & $60 \mathrm{C}$ SCW AQUEOUS PHASE \\
\hline IWA 102 & $\begin{array}{l}\text { A387 } \\
\text { Grade } 22\end{array}$ & 8760 & 28.713 & 3.3904 & 150.23 & $60 \mathrm{C}$ SCW WATERLINE \\
\hline WWH 077 & $\begin{array}{l}\text { A387 } \\
\text { Grade } 22\end{array}$ & 8760 & 29.134 & 2.8172 & 123.03 & $60 \mathrm{C}$ SCW VAPOR PHASE \\
\hline WH 078 & $\begin{array}{l}\text { A387 } \\
\text { Grade } 22\end{array}$ & 8760 & 29.326 & 3.6720 & 159.31 & $60 \mathrm{C}$ SCW VAPOR PHASE \\
\hline IWH 079 & $\begin{array}{l}\text { A387 } \\
\text { Grade } 22\end{array}$ & 8760 & 29.119 & 3.5753 & 156.21 & $60 \mathrm{C}$ SCW VAPOR PHASE \\
\hline IWH 080 & $\begin{array}{l}\text { A387 } \\
\text { Grade } 22\end{array}$ & 8760 & 27.938 & 0.1572 & 7.16 & $60 \mathrm{C}$ SCW AQUEOUS PHASE \\
\hline IWH OB1 & $\begin{array}{l}\text { A387 } \\
\text { Grade } 22\end{array}$ & 8760 & 29.456 & 0.1446 & 6.25 & $60 \mathrm{C}$ SCW AQUEOUS PHASE \\
\hline WH 082 & $\begin{array}{l}\text { A387 } \\
\text { Grade } 22\end{array}$ & 8760 & . $\quad 29.052$ & 0.1588 & 6.95 & $60 \mathrm{C}$ SCW AQUEOUS PHASE \\
\hline IWH 102 & $\begin{array}{l}\text { A387 } \\
\text { Grade } 22\end{array}$ & 8760 & 28.841 & 2.7097 & 119.53 & 60 C SCW WATERLINE \\
\hline JWA 077 & $\begin{array}{l}\text { A516 } \\
\text { Grade } 55\end{array}$ & 8760 & 29.304 & 3.6173 & 157.05 & $60 \mathrm{C}$ SCW VAPOR PHASE \\
\hline JWA 078 & $\begin{array}{l}\text { A516 } \\
\text { Grade } 55\end{array}$ & 8760 & 28.516 & 4.6929 & 209.38 & $60 \mathrm{C}$ SCW VAPOR PHASE \\
\hline JWA 079 & $\begin{array}{l}\text { A516 } \\
\text { Grade } 55\end{array}$ & 8760 & 28.717 & 4.8081 & 213.02 & $60 \mathrm{C} \mathrm{SCW}$ VAPOR PHASE \\
\hline JWA 080 & $\begin{array}{l}\text { A516 } \\
\text { Grade } 55\end{array}$ & 8760 & 29.549 & 0.1842 & 7.93 & $60 \mathrm{C}$ SCW AQUEOUS PHASE \\
\hline JWA 081 & $\begin{array}{l}\text { A516 } \\
\text { Grade } 55\end{array}$ & 8760 & 28.550 & 0.2510 & 11.19 & $60 \mathrm{C}$ SCW AQUEOUS PHASE \\
\hline
\end{tabular}




\section{Corrosion Rate Calculations for Weight Loss Specimens}

For Rack Number : 21-2

$\begin{array}{llll}\text { Sample Allov Lag Time Surface Area Weight Corrosion Rate Test Environment } \\ \text { Identification } & \text { (hours) (sq cm) Loss (g) (microns/year) }\end{array}$

\begin{tabular}{|c|c|c|c|c|c|c|}
\hline JWA 082 & $\begin{array}{l}\text { A516 } \\
\text { Grade } 55\end{array}$ & 8760 & 29.155 & 0.1841 & 8.03 & $60 \mathrm{C}$ SCW AQUEOUS PHASE \\
\hline JWA 102 & $\begin{array}{l}\text { A516 } \\
\text { Grade } 55\end{array}$ & 8760 & 28903 & 2.9941 & 131.80 & $60 \mathrm{C}$ SCW WATERLINE \\
\hline JWI 077 & $\begin{array}{l}\text { A.516 } \\
\text { Grade } 55\end{array}$ & 8760 & 28.236 & 4.4491 & 200.47 & 60 C SCW VAPOR PHASE \\
\hline JWI 078 & $\begin{array}{l}\text { A516 } \\
\text { Grade } 55\end{array}$ & 8760 & 28.039 & 5.8348 & 264.75 & $60 \mathrm{C}$ SCW VAPOR PHASE \\
\hline JWI 079 & $\begin{array}{l}\text { A516 } \\
\text { Grade } 55\end{array}$ & 8760 & 28.116 & 5.7048 & 258.15 & $60 \mathrm{C}$ SCW VAPOR PHASE \\
\hline JWI 080 & $\begin{array}{l}\text { A516 } \\
\text { Grade } 55\end{array}$ & 8760 & 27.937 & 0.2074 & 9.45 & $60 \mathrm{C}$ SCW AQUEOUS PHASE \\
\hline JWI 081 & $\begin{array}{l}\text { A516 } \\
\text { Grade } 55\end{array}$ & 8760 & 28.061 & 0.2438 & 11.05 & $60 \mathrm{C}$ SCW AQUEOUS PHASE \\
\hline JWI 082 & $\begin{array}{l}\text { A516 } \\
\text { Grade } 55\end{array}$ & 8760 & 27.753 & 0.1986 & 9.10 & $60 \mathrm{C}$ SCW AQUEOUS PHASE \\
\hline JWI 102 & $\begin{array}{l}\text { A516 } \\
\text { Grade } 55\end{array}$ & 8760 & 27.851 & 2.9865 & 136.42 & $60 \mathrm{C}$ SCW WATERLINE \\
\hline KWA 077 & $\begin{array}{l}\text { A27 Grade } \\
70-40\end{array}$ & 8760 & 27.867 & 1.6645 & 76.38 & $60 \mathrm{C}$ SCW VAPOR PHASE \\
\hline KWA 078 & $\begin{array}{l}\text { A27 Grade } \\
70-40\end{array}$ & 8760 & 28.008 & 1.9880 & 90.77 & $60 \mathrm{C}$ SCW VAPOR PHASE \\
\hline KWA 079 & $\begin{array}{l}\text { A27 Grade } \\
70-40\end{array}$ & 8760 & 28.271 & 2.8218 & 127.64 & $60 \mathrm{C}$ SCW VAPOR PHASE \\
\hline KWA 080 & $\begin{array}{l}\text { A27 Grade } \\
70-40\end{array}$ & 8760 & 28.298 & 0.2362 & 10.67 & $60 \mathrm{C}$ SCW AQUEOUS PHASE \\
\hline KWA 081 & $\begin{array}{l}\text { A27 Grade } \\
70-40\end{array}$ & 8760 & 27.882 & 0.2180 & 10.00 & $60 \mathrm{C}$ SCW AQUEOUS PHASE \\
\hline KWA 082 & $\begin{array}{l}\text { A27 Grade } \\
70-40\end{array}$ & 8760 & 27.934 & 0.1620 & 7.42 & $60 \mathrm{C}$ SCW AQUEOUS PHASE \\
\hline KWA 102 & $\begin{array}{l}\text { A27 Grade } \\
70-40\end{array}$ & 8760 & 28.196 & 3.9143 & 177.53 & 60 C SCW WATERLINE \\
\hline KWI 077 & $\begin{array}{l}\text { A27 Grade } \\
70-40\end{array}$ & 8760 & 28.532 & 1.7246 & 77.30 & $60 \mathrm{C}$ SCW VAPOR PHASE \\
\hline KWI 078 & $\begin{array}{l}\text { A27 Grade } \\
70-40\end{array}$ & 8760 & 28.328 & 1.7060 & 77.01 & $60 \mathrm{C}$ SCW VAPOR PHASE \\
\hline KWI 079 & $\begin{array}{l}\text { A27 Grade } \\
70-40\end{array}$ & 8760 & 28.203 & 2.1671 & 98.26 & $60 \mathrm{C}$ SCW VAPOR PHASE \\
\hline
\end{tabular}




\section{Corrosion Rate Calculations for Weight Loss Specimens}

For Rack Number : 21-2

\begin{tabular}{llccccc}
$\begin{array}{l}\text { Sample } \\
\text { Identification }\end{array}$ & Allov & $\begin{array}{c}\text { Lag Time Surface Area } \\
\text { (hours) }\end{array}$ & $\begin{array}{l}\text { Weight } \\
\text { (sq cm) }\end{array}$ & $\begin{array}{c}\text { Coss (g) } \\
\text { (microns/year) }\end{array}$ & \\
\hline KW1 080 & $\begin{array}{l}\text { A27 Grade } \\
70-40\end{array}$ & 8760 & 28.470 & 01801 & 8.09 & $60 \mathrm{C}$ SCW AQUEOUS PHASE \\
KWI 081 & $\begin{array}{l}\text { A27 Grade } \\
70-40\end{array}$ & 8760 & 28.080 & 0.2266 & 1032 & $60 \mathrm{C}$ SCW AQUEOUS PHASE \\
KW1 082 & $\begin{array}{l}\text { A27 Grade } \\
70-40\end{array}$ & 8760 & 28.611 & 0.2424 & 10.83 & $60 \mathrm{C}$ SCW AQUEOUS PHASE \\
KWI 102 & $\begin{array}{l}\text { A27 Grade } \\
70-40\end{array}$ & 8760 & 28.068 & 4.4400 & 202.28 & $60 \mathrm{C}$ SCW WATERLINE
\end{tabular}




\title{
Corrosion Rate Calculations for Weight Loss Specimens
} For Rack Number : 20-1

\author{
Sample Allov Lag Time Surface Area Weight Corrosion Rate Test Environment \\ Identification (hours) (sq cm) Loss (g) (microns/year)
}

\begin{tabular}{|c|c|c|c|c|c|c|}
\hline IWA 106 & $\begin{array}{l}\text { A387 } \\
\text { Grade } 22\end{array}$ & 4344 & 29.082 & 0.6845 & 60.39 & 90 C SCW WATERLINE \\
\hline WWA 111 & $\begin{array}{l}\text { A387 } \\
\text { Grade } 22\end{array}$ & 4344 & 28.709 & 1.0126 & 90.49 & $90 \mathrm{C}$ SCW VAPOR PHASE \\
\hline MA 112 & $\begin{array}{l}\text { A387 } \\
\text { Grade } 22\end{array}$ & 4344 & 29.008 & 1.3432 & 118.80 & $90 \mathrm{C}$ SCW VAPOR PHASE \\
\hline IWA 113 & $\begin{array}{l}\text { A387 } \\
\text { Grade } 22\end{array}$ & 4344 & 29.100 & 2.0452 & 180.31 & $90 \mathrm{C}$ SCW VAPOR PHASE \\
\hline IWA 114 & $\begin{array}{l}\text { A387 } \\
\text { Grade } 22\end{array}$ & 4344 & 28.950 & 0.1481 & 13.12 & $90 \mathrm{C}$ SCW AQUEOUS PHASE \\
\hline IWA 115 & $\begin{array}{l}\text { A387 } \\
\text { Grade } 22\end{array}$ & 4344 & 28.840 & 0.1323 & 11.77 & $90 \mathrm{C}$ SCW AQUEOUS PHASE \\
\hline IWA 116 & $\begin{array}{l}\text { A387 } \\
\text { Grade } 22\end{array}$ & 4344 & 28.746 & 0.1594 & 14.23 & $90 \mathrm{C}$ SCW AQUEOUS PHASE \\
\hline IWH 106 & $\begin{array}{l}\text { A387 } \\
\text { Grade } 22\end{array}$ & 4344 & 29.030 & 1.2430 & 109.85 & 90 C SCW WATERLINE \\
\hline WH 111 & $\begin{array}{l}\text { A387 } \\
\text { Grade } 22\end{array}$ & 4344 & 29.123 & 2.5059 & 220.76 & $90 \mathrm{C}$ SCW VAPOR PHASE \\
\hline WH 112 & $\begin{array}{l}\text { A387 } \\
\text { Grade } 22\end{array}$ & 4344 & 28.997 & 5.0985 & 451.11 & $90 \mathrm{C}$ SCW VAPOR PHASE \\
\hline WWH 113 & $\begin{array}{l}\text { A387 } \\
\text { Grade } 22\end{array}$ & 4344 & 29.143 & 0.5900 & 51.94 & $90 \mathrm{C}$ SCW VAPOR PHASE \\
\hline WH 114 & $\begin{array}{l}\text { A387 } \\
\text { Grade } 22\end{array}$ & 4344 & 29.289 & 0.1525 & 13.36 & $90 \mathrm{C}$ SCW AQUEOUS PHASE \\
\hline WH 115 & $\begin{array}{l}\text { A387 } \\
\text { Grade } 22\end{array}$ & 4344 & 29.124 & 0.1673 & 14.74 & $90 \mathrm{C}$ SCW AQUEOUS PHASE \\
\hline MH 116 & $\begin{array}{l}\text { A387 } \\
\text { Grade } 22\end{array}$ & 4344 & 29.036 & 0.1841 & 16.27 & $90 \mathrm{C}$ SCW AQUEOUS PHASE \\
\hline JWA 106 & $\begin{array}{l}\text { A516 } \\
\text { Grade } 55\end{array}$ & 4344 & 28.737 & 0.2115 & 18.88 & $90 \mathrm{C}$ SCW WATERLINE \\
\hline NWA 111 & $\begin{array}{l}\text { A516 } \\
\text { Grade } 55\end{array}$ & 4344 & 29.281 & 2.7599 & 241.82 & $90 \mathrm{C}$ SCW VAPOR PHASE \\
\hline JWA 112 & $\begin{array}{l}\text { A516 } \\
\text { Grade } 55\end{array}$ & 4344 & 28.245 & 3.5412 & 321.66 & $90 \mathrm{C}$ SCW VAPOR PHASE \\
\hline SWA 113 & $\begin{array}{l}\text { A516 } \\
\text { Grade } 55\end{array}$ & 4344 & 29.188 & 2.9348 & 257.97 & $90 \mathrm{C}$ SCW VAPOR PHASE \\
\hline SWA 114 & $\begin{array}{l}\text { A516 } \\
\text { Grade } 55\end{array}$ & 4344 & 29.374 & 0.1467 & 12.81 & $90 \mathrm{C}$ SCW AQUEOUS PHASE \\
\hline
\end{tabular}




\section{Corrosion Rate Calculations for Weight Loss Specimens}

For Rack Number : 20-1 $\begin{array}{llll}\text { Sample Allov } & \text { Lag Time Surface Area Weight Corrosion Rate Test Environment } \\ \text { Identification } & \text { (hours) } & (\mathrm{sq} \mathrm{cm}) & \text { Loss }(\mathrm{g}) \text { (microns/year) }\end{array}$

\begin{tabular}{|c|c|c|c|c|c|c|}
\hline JWA 115 & $\begin{array}{l}\text { A516 } \\
\text { Grade } 55\end{array}$ & 4344 & 29.539 & 0.1598 & 13.88 & $90 \mathrm{C}$ SCW AQUEOUS PHASE \\
\hline JWA 116 & $\begin{array}{l}\text { A516 } \\
\text { Grade } 55\end{array}$ & 4344 & 28644 & 0.1219 & 10.92 & $90 \mathrm{C}$ SCW AQUEOUS PHASE \\
\hline JWI 106 & $\begin{array}{l}\text { A516 } \\
\text { Grade } 55\end{array}$ & 4344 & 27.932 & 0.1359 & 12.48 & $90 \mathrm{C}$ SCW WATERLINE \\
\hline NWI 111 & $\begin{array}{l}\text { A516 } \\
\text { Grade } 55\end{array}$ & 4344 & 28.527 & 2.6476 & 238.11 & $90 \mathrm{C}$ SCW VAPOR PHASE \\
\hline JWI 112 & $\begin{array}{l}\text { A516 } \\
\text { Grade } 55\end{array}$ & 4344 & 27.735 & 2.1105 & 195.23 & $90 \mathrm{C}$ SCW VAPOR PHASE \\
\hline WI 113 & $\begin{array}{l}\text { A516 } \\
\text { Grade } 55\end{array}$ & 4344 & 27.902 & 2.0883 & 192.02 & $90 \mathrm{C}$ SCW VAPOR PHASE \\
\hline JWI 114 & $\begin{array}{l}\text { A516 } \\
\text { Grade } 55\end{array}$ & 4344 & 28.255 & 0.2101 & 19.08 & $90 \mathrm{C}$ SCW AQUEOUS PHASE \\
\hline JWI 115 & $\begin{array}{l}\text { A516 } \\
\text { Grade } 55\end{array}$ & 4344 & 27.951 & 0.1526 & 14.01 & $90 \mathrm{C}$ SCW AQUEOUS PHASE \\
\hline JWI 116 & $\begin{array}{l}\text { A516 } \\
\text { Grade } 55\end{array}$ & 4344 & 27.857 & 0.1759 & 16.20 & $90 \mathrm{C}$ SCW AQUEOUS PHASE \\
\hline KWA 106 & $\begin{array}{l}\text { A27 Grade } \\
70-40\end{array}$ & 4344 & 28.368 & 1.4599 & 132.71 & $90 \mathrm{C}$ SCW WATERLINE \\
\hline KWA 111 & $\begin{array}{l}\text { A27 Grade } \\
70-40\end{array}$ & 4344 & 28.161 & 1.8162 & 166.22 & $90 \mathrm{C}$ SCW VAPOR PHASE \\
\hline KWA 112 & $\begin{array}{l}\text { A27 Grade } \\
70-40\end{array}$ & 4344 & 28.287 & 2.8697 & 261.61 & $90 \mathrm{C}$ SCW VAPOR PHASE \\
\hline KWA 113 & $\begin{array}{l}\text { A27 Grade } \\
70-40\end{array}$ & 4344 & 28.207 & 2.2235 & 203.28 & $90 \mathrm{C}$ SCW VAPOR PHASE \\
\hline KWA 114 & $\begin{array}{l}\text { A27 Grade } \\
70-40\end{array}$ & 4344 & 28.114 & 0.1252 & 11.48 & $90 \mathrm{C}$ SCW AQUEOUS PHASE \\
\hline KWA 115 & $\begin{array}{l}\text { A27 Grade } \\
70-40\end{array}$ & 4344 & 28.044 & 0.1487 & 13.67 & $90 \mathrm{C}$ SCW AQUEOUS PHASE \\
\hline KWA 116 & $\begin{array}{l}\text { A27 Grade } \\
70-40\end{array}$ & 4344 & 27.879 & 0.0976 & 9.03 & $90 \mathrm{C}$ SCW AQUEOUS PHASE \\
\hline KWI 106 & $\begin{array}{l}\text { A27 Grade } \\
70-40\end{array}$ & 4344 & 27.973 & 0.6114 & 56.36 & $90 \mathrm{C}$ SCWWATERLINE \\
\hline KWI 111 & $\begin{array}{l}\text { A27 Grade } \\
70-40\end{array}$ & 4344 & 27.749 & 1.6176 & 150.33 & $90 \mathrm{C}$ SCW VAPOR PHASE \\
\hline$K W 1112$ & $\begin{array}{l}\text { A27 Grade } \\
70-40\end{array}$ & 4344 & 28.565 & 2.3328 & 210.60 & $90 \mathrm{C}$ SCW VAPOR PHASE \\
\hline
\end{tabular}




\section{Corrosion Rate Calculations for Weight Loss Specimens}

For Rack Number : 20-1

$\begin{array}{llll}\text { Sample Allov Lag Time Surface Area Weight Corrosion Rate Test Environment } \\ \text { Identification } & \text { (hours) } & \text { (sq cm) Loss (g) (microns/year) }\end{array}$

\begin{tabular}{llccccc}
\hline KWI 113 & $\begin{array}{l}\text { A27 Grade } \\
70-40\end{array}$ & 4344 & 27.301 & 3.3143 & 313.05 & $90 \mathrm{C}$ SCW VAPOR PHASE \\
KWI 114 & $\begin{array}{l}\text { A27 Grade } \\
70-40\end{array}$ & 4344 & 27188 & 01775 & 16.84 & $90 \mathrm{C}$ SCW AQUEOUS PHASE \\
KWI 115 & $\begin{array}{l}\text { A27 Grade } \\
70-40\end{array}$ & 4344 & 27.346 & 02339 & 22.06 & $90 \mathrm{C}$ SCW AQUEOUS PHASE \\
KWI 116 & $\begin{array}{l}\text { A27 Grade } \\
70-40\end{array}$ & 4344 & 27.602 & 0.1933 & 18.06 & $90 \mathrm{C}$ SCW AQUEOUS PHASE
\end{tabular}




\section{Corrosion Rate Calculations for Weight Loss Specimens} For Rack Number : 20-2
Sample Allov
Identification
Lag Time Surface Area Weight Corrosion Rate Test Environment
(hours) (sq cm) Loss (g) (micronsfyear)

\begin{tabular}{|c|c|c|c|c|c|c|}
\hline IWA 107 & $\begin{array}{l}\text { A387 } \\
\text { Grade } 22\end{array}$ & 8736 & 28840 & 2.6919 & 119.08 & $90 \mathrm{C}$ SCW WATERLINE \\
\hline IWA 117 & $\begin{array}{l}\text { A387 } \\
\text { Grade } 22\end{array}$ & 8736 & 28.818 & 5.6546 & 250.33 & $90 \mathrm{C}$ SCW VAPOR PHASE \\
\hline IWA 118 & $\begin{array}{l}\text { A387 } \\
\text { Grade } 22\end{array}$ & 8736 & 28.744 & 8.2820 & 367.59 & 90 C SCW VAPOR PHASE \\
\hline IWA 119 & $\begin{array}{l}\text { A387 } \\
\text { Grade } 22\end{array}$ & 8736 & 28.883 & 6.9939 & 308.92 & $90 \mathrm{C}$ SCW VAPOR PHASE \\
\hline IWA 120 & $\begin{array}{l}\text { A387 } \\
\text { Grade } 22\end{array}$ & 8736 & 28.807 & 0.1424 & 6.31 & $90 \mathrm{C}$ SCW AQUEOUS PHASE \\
\hline IWA 121 & $\begin{array}{l}\text { A387 } \\
\text { Grade } 22\end{array}$ & 8736 & 27.461 & 0.1594 & 7.41 & $90 \mathrm{C}$ SCW AQUEOUS PHASE \\
\hline IWA 122 & $\begin{array}{l}\text { A387 } \\
\text { Grade } 22\end{array}$ & 8736 & 28.838 & 0.1769 & 7.83 & $90 \mathrm{C}$ SCW AQUEOUS PHASE \\
\hline WWH 107 & $\begin{array}{l}\text { A387 } \\
\text { Grade } 22\end{array}$ & 8736 & 28.911 & 1.7789 & 78.50 & $90 \mathrm{C}$ SCW WATERLINE \\
\hline WH 117 & $\begin{array}{l}\text { A387 } \\
\text { Grade } 22\end{array}$ & 8736 & 28.958 & 5.3066 & 233.79 & $90 \mathrm{C}$ SCW VAPOR PHASE \\
\hline WH 118 & $\begin{array}{l}\text { A387 } \\
\text { Grade } 22\end{array}$ & 8736 & 29.164 & 9.3263 & 407.97 & $90 \mathrm{C}$ SCW VAPOR PHASE \\
\hline IWH 119 & $\begin{array}{l}\text { A387 } \\
\text { Grade } 22\end{array}$ & 8736 & 29.314 & 13.9834 & 608.57 & $90 \mathrm{C}$ SCW VAPOR PHASE \\
\hline IWH 120 & $\begin{array}{l}\text { A387 } \\
\text { Grade } 22\end{array}$ & 8736 & 29.035 & 0.0718 & 3.15 & $90 \mathrm{C}$ SCW AQUEOUS PHASE \\
\hline WH 121 & $\begin{array}{l}\text { A387 } \\
\text { Grade } 22\end{array}$ & 8736 & 29.172 & 0.0735 & 3.21 & $90 \mathrm{C}$ SCW AQUEOUS PHASE \\
\hline WH 122 & $\begin{array}{l}\text { A387 } \\
\text { Grade } 22\end{array}$ & 8736 & 29.043 & 0.0672 & 2.95 & $90 \mathrm{C}$ SCW AQUEOUS PHASE \\
\hline JWA 107 & $\begin{array}{l}\text { A516 } \\
\text { Grade } 55\end{array}$ & 8736 & 28.646 & 1.5234 & 67.85 & $90 \mathrm{C}$ SCW WATERLINE \\
\hline JWA 117 & $\begin{array}{l}\text { A516 } \\
\text { Grade } 55\end{array}$ & 8736 & 29.593 & 7.9562 & 342.99 & 90 C SCW VAPOR PHASE \\
\hline WWA 118 & $\begin{array}{l}\text { A516 } \\
\text { Grade } 55\end{array}$ & 8736 & 28.798 & 8.2022 & 363.36 & $90 \mathrm{C}$ SCW VAPOR PHASE \\
\hline IWA 120 & $\begin{array}{l}\text { A516 } \\
\text { Grade } 55\end{array}$ & 8736 & 28.625 & 0.1562 & 6.96 & $90 \mathrm{C}$ SCW AQUEOUS PHASE \\
\hline JWA 121 & $\begin{array}{l}\text { A516 } \\
\text { Grade } 55\end{array}$ & 8736 & 28.768 & 0.1297 & 5.75 & $90 \mathrm{C}$ SCW AQUEOUS PHASE \\
\hline
\end{tabular}




\section{Corrosion Rate Calculations for Weight Loss Specimens}

For Rack Number : 20-2

$\begin{array}{lll}\text { Sample Allov Lag Time Surface Area Weight Corrosion Rate Test Environment } \\ \text { Identification } & \text { (hours) (sq cm) Loss (g) (microns/year) }\end{array}$

\begin{tabular}{|c|c|c|c|c|c|c|}
\hline JWA 122 & $\begin{array}{l}\text { A516 } \\
\text { Grade } 55\end{array}$ & 8736 & 27.777 & 0.0803 & 3.69 & $90 \mathrm{C}$ SCW AQUEOUS PHASE \\
\hline JWI 107 & $\begin{array}{l}\text { A516 } \\
\text { Grade } 55\end{array}$ & 8736 & 28.068 & 1.0687 & 48.57 & $90 \mathrm{C}$ SCW WATERLINE \\
\hline JWI 117 & $\begin{array}{l}\text { A516 } \\
\text { Grade } 55\end{array}$ & 8736 & 28.037 & 7.8826 & 358.68 & $90 \mathrm{C}$ SCW VAPOR PHASE \\
\hline JWI 118 & $\begin{array}{l}\text { A516 } \\
\text { Grade } 55\end{array}$ & 8736 & 28.247 & 9.3670 & 423.06 & $90 \mathrm{C}$ SCW VAPOR PHASE \\
\hline JWI 119 & $\begin{array}{l}\text { A516 } \\
\text { Grade } 55\end{array}$ & 8736 & 27.511 & 4.1127 & 190.72 & $90 \mathrm{C}$ SCW VAPOR PHASE \\
\hline JWI 120 & $\begin{array}{l}\text { A516 } \\
\text { Grade } 55\end{array}$ & 8736 & 27.909 & 0.2045 & 9.35 & $90 \mathrm{C}$ SCW AQUEOUS PHASE \\
\hline JWI 121 & $\begin{array}{l}\text { A516 } \\
\text { Grade } 55\end{array}$ & 8736 & 27.763 & 0.1405 & 6.46 & $90 \mathrm{C}$ SCW AQUEOUS PHASE \\
\hline JWI 122 & $\begin{array}{l}\text { A516 } \\
\text { Grade } 55\end{array}$ & 8736 & 27.980 & 0.1502 & 6.85 & 9OC SCW AQUEOUS PHASE \\
\hline KWA 107 & $\begin{array}{l}\text { A27 Grade } \\
70-40\end{array}$ & 8736 & 28.159 & 2.6998 & 122.94 & $90 \mathrm{C}$ SCW WATERLINE \\
\hline KWA 117 & $\begin{array}{l}\text { A27 Grade } \\
70-40\end{array}$ & 8736 & 28.350 & 4.7050 & 212.81 & $90 \mathrm{C}$ SCW VAPOR PHASE \\
\hline KWA 118 & $\begin{array}{l}\text { A27 Grade } \\
70-40\end{array}$ & 8736 & 28.319 & 4.2686 & 193.28 & $90 \mathrm{C}$ SCW VAPOR PHASE \\
\hline KWA 119 & $\begin{array}{l}\text { A27 Grade } \\
70-40\end{array}$ & 8736 & 27.934 & 5.9267 & 272.06 & $90 \mathrm{C}$ SCW VAPOR PHASE \\
\hline KWA 120 & $\begin{array}{l}\text { A27 Grade } \\
70-40\end{array}$ & 8736 & 28.266 & 0.1435 & 6.51 & $90 \mathrm{C}$ SCW AQUEOUS PHASE \\
\hline KWA 121 & $\begin{array}{l}\text { A27 Grade } \\
70-40\end{array}$ & 8736 & 28.441 & 0.1582 & 7.13 & $90 \mathrm{C}$ SCW AQUEOUS PHASE \\
\hline KWA 122 & $\begin{array}{l}\text { A27 Grade } \\
70-40\end{array}$ & 8736 & 28.236 & 0.1171 & 5.32 & $90 \mathrm{C}$ SCW AQUEOUS PHASE \\
\hline KWI 107 & $\begin{array}{l}\text { A27 Grade } \\
70-40\end{array}$ & 8736 & 28.271 & 1.2315 & 55.86 & $90 \mathrm{C}$ SCW WATERLINE \\
\hline KWI 117 & $\begin{array}{l}\text { A27 Grade } \\
70-40\end{array}$ & 8736 & 28.232 & 1.7647 & 80.15 & $90 \mathrm{C}$ SCW VAPOR PHASE \\
\hline KWI 118 & $\begin{array}{l}\text { A27 Grade } \\
70-40\end{array}$ & 8736 & 27.786 & 2.6178 & 120.81 & $90 \mathrm{C}$ SCW VAPOR PHASE \\
\hline KWI 119 & $\begin{array}{l}\text { A27 Grade } \\
70-40\end{array}$ & 8736 & 28.015 & 3.6868 & 168.75 & $90 \mathrm{C}$ SCW VAPOR PHASE \\
\hline
\end{tabular}




\section{Corrosion Rate Calculations for Weight Loss Specimens}

For Rack Number : 20-2

Sample Allov Lag Time Surface Area Weight Corrosion Rate Test Environment

Identification (hours) (sq cm) Loss (g) (microns/year)

\begin{tabular}{lllllll}
\hline KWI 120 & $\begin{array}{l}\text { A27 Grade } \\
70-40\end{array}$ & 8736 & 27.819 & 01330 & 6.13 & $90 \mathrm{C}$ SCW AQUEOUS PHASE \\
KWI 121 & $\begin{array}{l}\text { A27 Grade } \\
70-40\end{array}$ & 8736 & 28.324 & 0.1305 & 5.91 & $90 \mathrm{C}$ SCW AQUEOUS PHASE \\
KWI 122 & $\begin{array}{l}\text { A27 Grade } \\
70-40\end{array}$ & 8736 & 27.476 & 0.1756 & 8.20 & $90 \mathrm{C}$ SCW AQUEOUS PHASE
\end{tabular}




\section{Corrosion Rate Calculations for Weight Loss Specimens}

For Rack Number : 18-1

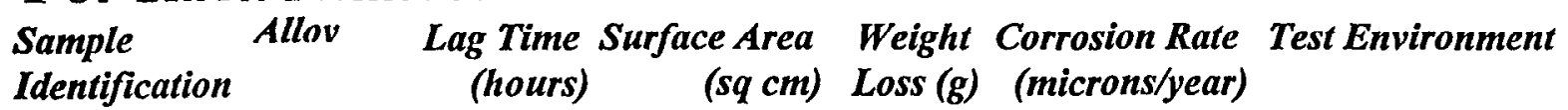

\begin{tabular}{|c|c|c|c|c|c|c|}
\hline GWA 036 & Monel 400 & 4392 & 28.017 & 0.7423 & 60.05 & $90 \mathrm{C}$ Waterline SAW \\
\hline GWA 041 & Monel 400 & 4392 & 28.755 & 0.0487 & 3.84 & $90 \mathrm{C}$ Vapor Phase SAW \\
\hline GWA 042 & Monel 400 & 4392 & 28.733 & 0.0568 & 4.48 & $90 \mathrm{C}$ Vapor Phase SAW \\
\hline GWA 043 & Monel 400 & 4392 & 27.942 & 0.0522 & 4.23 & $90 \mathrm{C}$ Vapor Phase SAW \\
\hline GWA 044 & Monel 400 & 4392 & 28.667 & 0.9861 & 77.96 & $90 \mathrm{C}$ Aqueous Phase SAW \\
\hline GWA 045 & Monel 400 & 4392 & 27.596 & 1.0208 & 83.84 & $90 \mathrm{C}$ Aqueous Phase SAW \\
\hline GWA 046 & Monel 400 & 4392 & 28.374 & 1.0802 & 86.29 & $90 \mathrm{C}$ Aqueous Phase SAW \\
\hline GWF 036 & Monel 400 & 4392 & 26.947 & 0.6858 & 57.68 & $90 \mathrm{C}$ Waterline SAW \\
\hline GWF 041 & Monel 400 & 4392 & 27.643 & 0.0480 & 3.94 & $90 \mathrm{C}$ Vapor Phase SAW \\
\hline GWF 042 & Monel 400 & 4392 & 27.490 & 0.0518 & 4.27 & $90 \mathrm{C}$ Vapor Phase SAW \\
\hline GWF 043 & Monel 400 & 4392 & 27.695 & 0.0424 & 3.47 & $90 \mathrm{C}$ Vapor Phase SAW \\
\hline GWF 044 & Monel 400 & 4392 & 26.870 & 1.0116 & 85.33 & $90 \mathrm{C}$ Aqueous Phase SAW \\
\hline GWF 045 & Monel 400 & 4392 & 27.399 & 1.0926 & 90.38 & $90 \mathrm{C}$ Aqueous Phase SAW \\
\hline GWF 0.46 & Monel 400 & 4392 & 27.275 & 1.1571 & 96.15 & $90 \mathrm{C}$ Aqueous Phase SAW \\
\hline HWA 036 & CDA 715 & 4392 & 27.788 & 3.5809 & 287.50 & 90 C Waterline SAW \\
\hline HWA 041 & CDA 715 & 4392 & 27.795 & 0.2227 & 17.88 & $90 \mathrm{C}$ Vapor Phase SAW \\
\hline HWA 042 & CDA 715 & 4392 & 27.643 & 0.1531 & 12.36 & $90 \mathrm{C}$ Vapor Phase SAW \\
\hline HWA 043 & $\operatorname{CDA} 715$ & 4392 & 27.723 & 0.1116 & 8.98 & $90 \mathrm{C}$ Vapor Phase SAW \\
\hline HWA 044 & CDA 715 & 4392 & 28.262 & 3.2813 & 259.03 & $90 \mathrm{C}$ Aqueous Phase SAW \\
\hline
\end{tabular}




\section{Corrosion Rate Calculations for Weight Loss Specimens}

For Rack Number : 18-1

\begin{tabular}{|c|c|c|c|c|c|c|}
\hline $\begin{array}{l}\text { Sample } \\
\text { Identification }\end{array}$ & Allov & $\begin{array}{r}\text { Lag Time } \\
\text { (hours) }\end{array}$ & $\begin{array}{r}\text { Surface Area } \\
(\mathrm{sq} \mathrm{cm})\end{array}$ & $\begin{array}{l}\text { Weight } \\
\text { Loss (g) }\end{array}$ & $\begin{array}{c}\text { Corrosion Rate } \\
\text { (microns/year) }\end{array}$ & Test Environment \\
\hline HWA 045 & $\operatorname{CDA} 715$ & 4392 & 27.981 & 3.2059 & 255.62 & $90 \mathrm{C}$ Aqueous Phase SAW \\
\hline HWA 046 & CDA 715 & 4392 & 27.482 & 3.6026 & 292.46 & $90 \mathrm{C}$ Aqueous Phase SAW \\
\hline HWG 036 & CDA 715 & 4392 & 26.834 & 3.3935 & 282.14 & $90 \mathrm{C}$ Waterline SAW \\
\hline HWG 041 & CDA 715 & 4392 & 26.815 & 0.1346 & 11.20 & $90 \mathrm{C}$ Vapor Phase SAW \\
\hline HWG 042 & CDA 715 & 4392 & 26.923 & 0.1047 & 8.68 & $90 \mathrm{C}$ Vapor Phase SAW \\
\hline HWG 043 & CDA 715 & 4392 & 26.822 & 0.0953 & 7.93 & 90 C Vapor Phase SAW \\
\hline HWG 044 & CDA 715 & 4392 & 26.749 & 3.4887 & 290.97 & $90 \mathrm{C}$ Aqueous Phase SAW \\
\hline HWG 045 & CDA 715 & 4392 & 26.907 & 3.6809 & 305.21 & $90 \mathrm{C}$ Aqueous Phase SAW \\
\hline HWG 046 & $\operatorname{CDA} 715$ & 4392 & 26.842 & 3.7686 & 313.24 & $90 \mathrm{C}$ Aqueous Phase SAW \\
\hline
\end{tabular}




\section{Corrosion Rate Calculations for Weight Loss Specimens}

For Rack Number : 18-2

Sample Allov Lag Time Surface Area Weight Corrosion Rate Test Environment Identification (hours) (sq cm) Loss (g) (microns/year)

\begin{tabular}{|c|c|c|c|c|c|c|}
\hline GWA 037 & Monel 400 & 8760 & 27.930 & 0.8876 & 36.11 & $90 \mathrm{C}$ Waterline SAW \\
\hline GWA 047 & Monel 400 & 8760 & 28.417 & 0.0491 & 1.96 & 90 C Vapor Phase SAW \\
\hline GWA 048 & Monel 400 & 8760 & 28.258 & 0.0504 & 2.03 & $90 \mathrm{C}$ Vapor Phase SAW \\
\hline GWA 049 & Monel 400 & 8760 & 27.905 & 0.0580 & 2.36 & $90 \mathrm{C}$ Vapor Phase SAW \\
\hline GWA 050 & Monel 400 & 8760 & 28.608 & 1.1329 & 45.00 & $90 \mathrm{C}$ Aqueous Phase SAW \\
\hline GWA 051 & Monel 400 & 8760 & 27.844 & 1.1457 & 46.76 & $90 \mathrm{C}$ Aqueous Phase SAW \\
\hline GWA 052 & Monel 400 & 8760 & 28.658 & 1.1759 & 46.63 & $90 \mathrm{C}$ Aqueous Phase SAW \\
\hline GWF 037 & Monel 400 & 8760 & 27.380 & 0.9459 & 39.26 & $90 \mathrm{C}$ Waterline SAW \\
\hline GWF 047 & Monel 400 & 8760 & 27.287 & 0.0560 & 2.33 & $90 \mathrm{C}$ Vapor Phase SAW \\
\hline GWF 048 & Monel 400 & 8760 & 26.898 & 0.0460 & 1.94 & $90 \mathrm{C}$ Vapor Phase SAW \\
\hline GWF 049 & Monel 400 & 8760 & 27.300 & 0.0526 & 2.19 & $90 \mathrm{C}$ Vapor Phase SAW \\
\hline GWF 050 & Monel 400 & 8760 & 27.645 & 1.1954 & 49.14 & $90 \mathrm{C}$ Aqueous Phase SAW \\
\hline GWF 051 & Monel 400 & 8760 & 27.661 & 1.2342 & 50.70 & $90 \mathrm{C}$ Aqueous Phase SAW \\
\hline GWF 052 & Monel 400 & 8760 & 27.268 & 1.2562 & 52.35 & $90 \mathrm{C}$ Aqueous Phase SAW \\
\hline HWA 037 & CDA 715 & 8760 & 27.950 & 6.1186 & 244.87 & 90 C Waterline SAW \\
\hline HWA 047 & CDA 715 & 8760 & 27.856 & 0.3061 & 12.29 & $90 \mathrm{C}$ Vapor Phase SAW \\
\hline HWA 048 & $\operatorname{CDA} 715$ & 8760 & 27.943 & 0.2586 & 10.35 & $90 \mathrm{C}$ Vapor Phase SAW \\
\hline HWA 049 & CDA 715 & 8760 & 28.221 & 0.1936 & 7.67 & $90 \mathrm{C}$ Vapor Phase SAW \\
\hline HWA 050 & CDA 715 & 8760 & 28.120 & 4.2182 & 167.79 & $90 \mathrm{C}$ Aqueous Phase SAW \\
\hline
\end{tabular}




\section{Corrosion Rate Calculations for Weight Loss Specimens}

For Rack Number : 18-2

\begin{tabular}{lcccccc}
$\begin{array}{l}\text { Sample } \\
\text { Identification }\end{array}$ & Allov & $\begin{array}{c}\text { Lag Time Surface Area } \\
\text { (hours) }\end{array}$ & $\begin{array}{l}\text { Weight } \\
\text { (sq cm) }\end{array}$ & $\begin{array}{c}\text { Coss }(g) \\
\text { (microns/year) }\end{array}$ & \\
\hline HWA 051 & CDA 715 & 8760 & 27.779 & 6.0110 & 242.05 & 90 C Aqueous Phase SAW \\
HWA 052 & CDA 715 & 8760 & 27.538 & 3.8218 & 155.24 & 90 C Aqueous Phase SAW \\
HWG 037 & CDA 715 & 8760 & 26.767 & 4.7066 & 196.68 & 90 C Waterline SAW \\
HWG 047 & CDA 715 & 8760 & 26.890 & 0.2696 & 11.21 & 90 C Vapor Phase SAW \\
HWG 048 & CDA 715 & 8760 & 26.606 & 0.2005 & 8.43 & 90 C Vapor Phase SAW \\
HWG 049 & CDA 715 & 8760 & 26.350 & 0.1964 & 8.34 & 90 C Vapor Phase SAW \\
HWG 050 & CDA 715 & 8760 & 26.781 & 5.2821 & 220.62 & 90 C Aqueous Phase SAW \\
HWG 051 & CDA 715 & 8760 & 26.842 & 5.4112 & 225.50 & 90 C Aqueous Phase SAW \\
HWG 052 & CDA 715 & 8760 & 26.785 & 5.4941 & 229.43 & 90 C Aqueous Phase SAW
\end{tabular}




\section{Corrosion Rate Calculations for Weight Loss Specimens}

For Rack Number : 19-1

\section{Sample Allov Lag Time Surface Area Weight Corrosion Rate Test Environment Identification (hours) (sq cm) Loss (g) (microns/year)}

\begin{tabular}{|c|c|c|c|c|c|c|}
\hline GWA 001 & Monel 400 & 4536 & 28492 & 0.0061 & 047 & $60 \mathrm{C}$ Vapor Phase SAW \\
\hline GWA 002 & Monel 400 & 4536 & 28198 & 0.0117 & 0.91 & $60 \mathrm{C}$ Vapor Phase SAW \\
\hline GWA 003 & Monel 400 & 4536 & 28.388 & 0.0153 & 1.18 & $60 \mathrm{C}$ Vapor Phase SAW \\
\hline GWA 004 & Monel 400 & 4536 & 28.400 & 1.4796 & 114.33 & $60 \mathrm{C}$ Aqueous Phase SAW \\
\hline GWA 005 & Monel 400 & 4536 & 27.931 & 1.4297 & 112.33 & $60 \mathrm{C}$ Aqueous Phase SAW \\
\hline GWA 006 & Monel 400 & 4536 & 28.379 & 1.5462 & 119.57 & $60 \mathrm{C}$ Aqueous Phase SAW \\
\hline GWA 031 & Monel 400 & 4536 & 27.706 & 1.0818 & 85.69 & $60 \mathrm{C}$ Water Line SAW \\
\hline GWF 001 & Monel 400 & 4536 & 27.166 & 0.0107 & 0.86 & 60 C Vapor Phase SAW \\
\hline GWF 002 & Monel 400 & 4536 & 27.768 & 0.0143 & 1.13 & $60 \mathrm{C}$ Vapor Phase SAW \\
\hline GWF 003 & Monel 400 & 4536 & 27.774 & 0.0129 & 1.02 & $60 \mathrm{C}$ Vapor Phase SAW \\
\hline GWF 004 & Monel 400 & 4536 & 27.532 & 1.4721 & 117.34 & $60 \mathrm{C}$ Aqueous Phase SAW \\
\hline GWF 005 & Monel 400 & 4536 & 27.486 & 1.5264 & 121.87 & $60 \mathrm{C}$ Aqueous Phase SAW \\
\hline GWF 006 & Monel 400 & 4536 & 27.178 & 1.5832 & 127.84 & $60 \mathrm{C}$ Aqueous Phase SAW \\
\hline GWF 031 & Monel 400 & 4536 & 27.124 & 1.0996 & 88.97 & $60 \mathrm{C}$ Water Line SAW \\
\hline HWA 001 & CDA 715 & 4536 & 27.506 & 0.0402 & 3.16 & 60 C Vapor Phase SAW \\
\hline HWA 002 & CDA 715 & 4536 & 27.585 & 0.0543 & 4.25 & $60 \mathrm{C}$ Vapor Phase SAW \\
\hline HWA 003 & CDA 715 & 4536 & 28.221 & 0.0618 & 4.73 & $60 \mathrm{C}$ Vapor Phase SAW \\
\hline HWA 004 & CDA 715 & 4536 & 27.845 & 3.2743 & 254.02 & $60 \mathrm{C}$ Aqueous Phase SAW \\
\hline HWA 005 & CDA 715 & 4536 & 27.870 & 3.4426 & 266.83 & $60 \mathrm{C}$ Aqueous Phase SAW \\
\hline
\end{tabular}




\section{Corrosion Rate Calculations for Weight Loss Specimens}

For Rack Number : 19-1

\begin{tabular}{|c|c|c|c|c|c|c|}
\hline $\begin{array}{l}\text { Sample } \\
\text { Identification }\end{array}$ & Allov & $\begin{array}{c}\text { Lag Time } \\
\text { (hours) }\end{array}$ & $\begin{array}{r}\text { Surface Area } \\
(\mathrm{sq} \mathrm{cm})\end{array}$ & $\begin{array}{l}\text { Weight } \\
\text { Loss (g) }\end{array}$ & $\begin{array}{l}\text { Corrosion Rate } \\
\text { (microns/year) }\end{array}$ & Test Environment \\
\hline HWA 006 & CDA 715 & 4536 & 27.914 & 3.7590 & 290.90 & $60 \mathrm{C}$ Aqueous Phase SAW \\
\hline HWA 031 & CDA 715 & 4536 & 27.476 & 4.7230 & 371.32 & $60 \mathrm{C}$ Water Line SAW \\
\hline HWG 001 & CDA 715 & 4536 & 26.810 & 0.0435 & 3.50 & $60 \mathrm{C}$ Vapor Phase SAW \\
\hline HWG 002 & CDA 715 & 4536 & 26.888 & 0.0484 & 3.89 & 60 C Vapor Phase SAW \\
\hline HWG 003 & CDA 715 & 4536 & 26.884 & 0.0660 & 5.30 & 60 C Vapor Phase SAW \\
\hline HWG 004 & CDA 715 & 4536 & 26.743 & 2.5337 & 204.66 & $60 \mathrm{C}$ Aqueous Phase SAW \\
\hline HWG 005 & CDA 715 & 4536 & 26.661 & 2.7715 & 224.56 & $60 \mathrm{C}$ Aqueous Phase SAW \\
\hline HWG 006 & CDA 715 & 4536 & 26.533 & 3.1442 & 255.99 & $60 \mathrm{C}$ Aqueous Phase SAW \\
\hline HWG 031 & CDA 715 & 4536 & 26.826 & 5.3929 & 434.28 & $60 \mathrm{C}$ Water Line SAW \\
\hline
\end{tabular}




\section{Corrosion Rate Calculations for Weight Loss Specimens}

For Rack Number : 19-2

Sample Allov Lag Time Surface Area Weight Corrosion Rate Test Environment Identification (hours) (sq cm) Loss (g) (microns/year)

\begin{tabular}{|c|c|c|c|c|c|c|}
\hline GWA 007 & Monel 400 & 8760 & 28.042 & 0.0110 & 0.45 & $60 \mathrm{C}$ Vapor Phase SAW \\
\hline GWA 008 & Monel 400 & 8760 & 27.977 & 0.0119 & 0.48 & $60 \mathrm{C}$ Vapor Phase SAW \\
\hline GWA 009 & Monel 400 & 8760 & 28.431 & 0.0108 & 0.43 & $60 \mathrm{C}$ Vapor Phase SAW \\
\hline GWA 010 & Monel 400 & 8760 & 28.531 & 1.6761 & 66.76 & $60 \mathrm{C}$ Aqueous Phase SAW \\
\hline GWA 011 & Monel 400 & 8760 & 28.506 & 1.6655 & 66.39 & $60 \mathrm{C}$ Aqueous Phase SAW \\
\hline GWA 012 & Monel 400 & 8760 & 27.989 & 1.6915 & 68.68 & $60 \mathrm{C}$ Aqueous Phase SAW \\
\hline GWA 032 & Monel 400 & 8760 & 27.750 & 1.3017 & 53.30 & $60 \mathrm{C}$ Water Line SAW \\
\hline GWF 007 & Monel 400 & 8760 & 27.286 & 0.0185 & 0.77 & $60 \mathrm{C}$ Vapor Phase SAW \\
\hline GWF 008 & Monel 400 & 8760 & 27.800 & 0.0337 & 1.38 & $60 \mathrm{C}$ Vapor Phase SAW \\
\hline GWF 009 & Monel 400 & 8760 & 27.765 & 0.0319 & 1.31 & $60 \mathrm{C}$ Vapor Phase SAW \\
\hline GWF 010 & Monel 400 & 8760 & 27.852 & 1.5732 & 64.19 & $60 \mathrm{C}$ Aqueous Phase SAW \\
\hline GWF 011 & Monel 400 & 8760 & 27.381 & 1.5294 & 63.47 & $60 \mathrm{C}$ Aqueous Phase SAW \\
\hline GWF 012 & Monel 400 & 8760 & 27.162 & 1.5600 & 65.26 & $60 \mathrm{C}$ Aqueous Phase SAW \\
\hline GWF 032 & Monel 400 & 8760 & 27.968 & 1.3393 & 54.42 & $60 \mathrm{C}$ Water Line SAW \\
\hline HWA 007 & CDA 715 & 8760 & 27.707 & 0.0814 & 3.29 & $60 \mathrm{C}$ Vapor Phase SAW \\
\hline HWA 008 & CDA 715 & 8760 & 27.800 & 0.0895 & 3.60 & 60 C Vapor Phase SAW \\
\hline HWA 009 & CDA 715 & 8760 & 27.773 & 0.0993 & 4.00 & 60 C Vapor Phase SAW \\
\hline HWA 010 & CDA 715 & 8760 & 27.990 & 3.3825 & 135.18 & $60 \mathrm{C}$ Aqueous Phase SAW \\
\hline HWA 011 & CDA 715 & 8760 & 27.332 & 3.3421 & 136.77 & $60 \mathrm{C}$ Aqueous Phase SAW \\
\hline
\end{tabular}




\section{Corrosion Rate Calculations for Weight Loss Specimens}

For Rack Number : 19-2

\begin{tabular}{|c|c|c|c|c|c|c|}
\hline $\begin{array}{l}\text { Sample } \\
\text { Identification }\end{array}$ & Allov & $\begin{array}{r}\text { Lag Time } \\
\text { (hours) }\end{array}$ & $\begin{array}{r}\text { Surface Area } \\
(\mathrm{sq} \mathrm{cm})\end{array}$ & $\begin{array}{l}\text { Weight } \\
\text { Loss (g) }\end{array}$ & $\begin{array}{l}\text { Corrosion Rate } \\
\text { (microns/year) }\end{array}$ & Test Environment \\
\hline HWA 012 & CDA 715 & 8760 & 27.574 & 3.5737 & 144.97 & $60 \mathrm{C}$ Aqueous Phase SAW \\
\hline HWA 032 & CDA 715 & 8760 & 27.652 & 5.4566 & 220.73 & $60 \mathrm{C}$ Water Line SAW \\
\hline HWG 007 & CDA 715 & 8760 & 26.725 & 0.0562 & 2.35 & $60 \mathrm{C}$ Vapor Phase SAW \\
\hline HWG 008 & CDA 715 & 8760 & 26.743 & 0.0939 & 3.93 & $60 \mathrm{C}$ Vapor Phase SAW \\
\hline HWG 009 & CDA 715 & 8760 & 26.805 & 0.1004 & 4.19 & 60 C Vapor Phase SAW \\
\hline HWG 010 & CDA 715 & 8760 & 26.563 & 2.6060 & 109.74 & $60 \mathrm{C}$ Aqueous Phase SAW \\
\hline HWG 011 & CDA 715 & 8760 & 26.830 & 2.7953 & 116.54 & $60 \mathrm{C}$ Aqueous Phase SAW \\
\hline HWG 012 & CDA 715 & 8760 & 26.733 & 3.0622 & 128.13 & $60 \mathrm{C}$ Aqueous Phase SAW \\
\hline HWG 032 & $\operatorname{CDA} 715$ & 8760 & 26.890 & 50582 & 210.41 & $60 \mathrm{C}$ Water Line SAW \\
\hline
\end{tabular}




\section{Corrosion Rate Calculations for Weight Loss Specimens}

For Rack Number : 25-1

Sample Allov Lag Time Surface Area Weight Corrosion Rate Test Environment Identification (hours) (sq cm) Loss (g) (microns/year)

\begin{tabular}{|c|c|c|c|c|c|c|}
\hline AWA 001 & 1825 & 4296 & 28.303 & -0.0005 & -0.04 & 60 C Vapor Phase SAW \\
\hline AWA 002 & 1825 & 4296 & 28.375 & 0.0000 & 0.00 & 60 C Vapor Phase SAW \\
\hline AWA 003 & 1825 & 4296 & 28.342 & 0.0003 & 003 & $60 \mathrm{C}$ Vapor Phase SAW \\
\hline AWA 004 & 1825 & 4296 & 28.264 & -0.0002 & -0.02 & $60 \mathrm{C}$ Aqueous Phase SAW \\
\hline AWA 005 & 1825 & 4296 & 28.212 & -0.0003 & -0.03 & $60 \mathrm{C}$ Aqueous Phase SAW \\
\hline AWA 006 & 1825 & 4296 & 27.812 & 0.0015 & 0.14 & $60 \mathrm{C}$ Aqueous Phase SAW \\
\hline AWA 031 & 1825 & 4296 & 28.153 & -0.0002 & -0.02 & $60 \mathrm{C}$ Water Line SAW \\
\hline AWB 001 & 1825 & 4296 & 27.736 & -0.0004 & -0.04 & 60 C Vapor Phase SAW \\
\hline AWB 002 & 1825 & 4296 & 26.746 & 0.0010 & 0.09 & 60 C Vapor Phase SAW \\
\hline AWB 003 & 1825 & 4296 & 27.529 & 0.0004 & 0.04 & 60 C Vapor Phase SAW \\
\hline AWB 004 & 1825 & 4296 & 27.560 & -0.0003 & -0.03 & $60 \mathrm{C}$ Aqueous Phase SAW \\
\hline AWB 005 & 1825 & 4296 & 27.533 & 0.0001 & 0.01 & $60 \mathrm{C}$ Aqueous Phase SAW \\
\hline AWB 006 & 1825 & 4296 & 27.539 & -0.0011 & -0.10 & $60 \mathrm{C}$ Aqueous Phase SAW \\
\hline AWB 031 & 1825 & 4296 & 27.565 & -0.0006 & -0.05 & 60 C Water Line SAW \\
\hline BWA 001 & G3 & 4296 & 28.307 & -0.0003 & -0.03 & 60 C Vapor Phase SAW \\
\hline BWA 002 & G3 & 4296 & 28.325 & -0.0006 & -0.05 & $60 \mathrm{C}$ Vapor Phase SAW \\
\hline BWA 003 & G3 & 4296 & 28.545 & -0.0002 & -0.02 & $60 \mathrm{C}$ Vapor Phase SAW \\
\hline BWA 004 & G3 & 4296 & 28.738 & -0.0007 & -0.06 & $60 \mathrm{C}$ Aqueous Phase SAW \\
\hline BWA 005 & G3 & 4296 & 28.257 & -0.0001 & -0.01 & $60 \mathrm{C}$ Aqueous Phase SAW \\
\hline
\end{tabular}




\section{Corrosion Rate Calculations for Weight Loss Specimens}

For Rack Number : 25-1

\begin{tabular}{|c|c|c|c|}
\hline $\begin{array}{l}\text { Sample Allov } \\
\text { Identification }\end{array}$ & $\begin{array}{c}\text { Lag Time Surface Area } \\
\text { (hours) }\end{array}$ & $\begin{array}{l}\text { Weight Corrosion Rate } \\
\text { Loss (g) (microns/year) }\end{array}$ & Test Environment \\
\hline
\end{tabular}

\begin{tabular}{|c|c|c|c|c|c|c|}
\hline BWA 006 & G3 & 4296 & 28.448 & -0.0023 & -020 & $60 \mathrm{C}$ Aqueous Phase SAW \\
\hline BWA 031 & G3 & 4296 & 28.547 & -0.0004 & -0.03 & $60 \mathrm{C}$ Water Line SAW \\
\hline BWC 001 & G3 & 4296 & 28.347 & -0.0004 & -0.03 & $60 \mathrm{C}$ Vapor Phase SAW \\
\hline BWC 002 & G3 & 4296 & 27.942 & 0.0000 & 0.00 & $60 \mathrm{C}$ Vapor Phase SAW \\
\hline BWC 003 & G3 & 4296 & 28.262 & -0.0005 & -0.04 & $60 \mathrm{C}$ Vapor Phase SAW \\
\hline BWC 004 & G3 & 4296 & 28.154 & -0.0005 & -0.04 & $60 \mathrm{C}$ Aqueous Phase SAW \\
\hline BWC 005 & G3 & 4296 & 27.945 & -0.0002 & -0.02 & $60 \mathrm{C}$ Aqueous Phase SAW \\
\hline BWC 006 & G3 & 4296 & 28.293 & -0.0002 & -0.02 & $60 \mathrm{C}$ Aqueous Phase SAW \\
\hline BWC 031 & G3 & 4296 & 28.296 & -0.0002 & -0.02 & $60 \mathrm{C}$ Water Line SAW \\
\hline CWA 001 & C4 & 4296 & 28.012 & 0.0002 & 0.02 & $60 \mathrm{C}$ Vapor Phase SAW \\
\hline CWA 002 & $\mathrm{C} 4$ & 4296 & 27.868 & 0.0000 & 0.00 & $60 \mathrm{C}$ Vapor Phase SAW \\
\hline CWA 003 & C4 & 4296 & 27.964 & 0.0001 & 0.01 & $60 \mathrm{C}$ Vapor Phase SAW \\
\hline CWA 004 & C4 & 4296 & 27.812 & 0.0005 & 0.04 & $60 \mathrm{C}$ Aqueous Phase SAW \\
\hline CWA 005 & $\mathrm{C4}$ & 4296 & 27.819 & 0.0004 & 0.03 & $60 \mathrm{C}$ Aqueous Phase SAW \\
\hline CWA 006 & $\mathrm{C}_{4}$ & 4296 & 28.082 & 0.0005 & 0.04 & $60 \mathrm{C}$ Aqueous Phase SAW \\
\hline CWA 031 & $\mathrm{C}_{4}$ & 4296 & 28.337 & 0.0002 & 0.02 & $60 \mathrm{C}$ Water Line SAW \\
\hline CWB 001 & $\mathrm{C}_{4}$ & 4296 & 27.780 & 0.0001 & 0.01 & $60 \mathrm{C}$ Vapor Phase SAW \\
\hline CWB 002 & C4 & 4296 & 27.118 & 0.0001 & 0.01 & $60 \mathrm{C}$ Vapor Phase SAW \\
\hline CWB 003 & $\mathrm{C}_{4}$ & 4296 & 27.445 & 0.0007 & 0.06 & 60 C Vapor Phase SAW \\
\hline
\end{tabular}




\section{Corrosion Rate Calculations for Weight Loss Specimens}

For Rack Number : 25-1 $\begin{array}{llrl}\text { Sample Allov Lag Time Surface Area } & \text { Weight Corrosion Rate Test Environment } \\ \text { Identification } & \text { (hours) } & \text { (sq cm) Loss (g) } & \text { (microns/year) }\end{array}$

\begin{tabular}{|c|c|c|c|c|c|c|}
\hline CWB 004 & $\mathrm{C} 4$ & 4296 & 28.020 & -0.0001 & -0.01 & $60 \mathrm{C}$ Aqueous Phase SAW \\
\hline CWB 005 & $\mathrm{C} 4$ & 4296 & 27.769 & 0.0006 & 0.05 & $60 \mathrm{C}$ Aqueous Phase SAW \\
\hline CWB 006 & C4 & 4296 & 27.727 & -0.0003 & -0.03 & $60 \mathrm{C}$ Aqueous Phase SAW \\
\hline CWB 031 & $\mathrm{C} 4$ & 4296 & 27.695 & -0.0003 & -0.03 & $60 \mathrm{C}$ Water Line SAW \\
\hline DWA 001 & $\mathrm{C} 22$ & 4296 & 28.168 & 0.0003 & 0.03 & 60 C Vapor Phase SAW \\
\hline DWA 002 & $\mathrm{C} 22$ & 4296 & 28.149 & -0.0006 & -0.05 & $60 \mathrm{C}$ Vapor Phase SAW \\
\hline DWA 003 & $\mathrm{C} 22$ & 4296 & 28.040 & 0.0006 & 0.05 & $60 \mathrm{C}$ Vapor Phase SAW \\
\hline DWA 004 & $\mathrm{C} 22$ & 4296 & 28.019 & -0.0007 & -0.06 & $60 \mathrm{C}$ Aqueous Phase SAW \\
\hline DWA 005 & $\mathrm{C} 22$ & 4296 & 28.027 & -0.0004 & -0.03 & $60 \mathrm{C}$ Aqueous Phase SAW \\
\hline DWA 006 & $\mathrm{C} 22$ & 4296 & 28.222 & -0.0003 & -0.03 & $60 \mathrm{C}$ Aqueous Phase SAW \\
\hline DWA 031 & $\mathrm{C} 22$ & 4296 & 27.775 & -0.0004 & -0.03 & $60 \mathrm{C}$ Water Line SAW \\
\hline DWB 001 & $\mathrm{C} 22$ & 4296 & 27.388 & 0.0013 & 0.11 & $60 \mathrm{C}$ Vapor Phase SAW \\
\hline DWB 002 & $\mathrm{C} 22$ & 4296 & 27.443 & 0.0019 & 0.16 & $60 \mathrm{C}$ Vapor Phase SAW \\
\hline DWB 003 & C22 & 4296 & 27.536 & 0.0007 & 0.06 & $60 \mathrm{C}$ Vapor Phase SAW \\
\hline DWB 004 & C22 & 4296 & 27.490 & 0.0000 & 0.00 & $60 \mathrm{C}$ Aqueous Phase SAW \\
\hline DWB 005 & C22 & 4296 & 27.462 & 0.0004 & 0.03 & $60 \mathrm{C}$ Aqueous Phase SAW \\
\hline DWB 006 & C22 & 4296 & 27.322 & 0.0004 & 0.03 & $60 \mathrm{C}$ Aqueous Phase SAW \\
\hline DWB 031 & C22 & 4296 & 27.605 & -0.0004 & -0.03 & $60 \mathrm{C}$ Water Line SAW \\
\hline EWA 001 & Ti Grade 12 & 4296 & 28.243 & 0.0308 & 5.02 & $60 \mathrm{C}$ Vapor Phase SAW \\
\hline
\end{tabular}




\section{Corrosion Rate Calculations for Weight Loss Specimens} For Rack Number : 25-1
Sample
Allov
Lag Time Surface Area
Weight Corrosion Rate
(hours)
(sq cm) Loss (g) (microns/year)

\begin{tabular}{|c|c|c|c|c|c|c|}
\hline EWA 002 & Ti Grade 12 & 4296 & 28.928 & 0.0310 & 4.93 & $60 \mathrm{C}$ Vapor Phase SAW \\
\hline EWA 003 & Ti Grade 12 & 4296 & 28.644 & 0.0278 & 4.47 & $60 \mathrm{C}$ Vapor Phase SAW \\
\hline EWA 004 & Ti Grade 12 & 4296 & 28.295 & 0.0230 & 3.74 & $60 \mathrm{C}$ Aqueous Phase SAW \\
\hline EWA 005 & Ti Grade 12 & 4296 & 28.719 & 0.0254 & 4.07 & $60 \mathrm{C}$ Aqueous Phase SAW \\
\hline EWA 006 & Ti Grade 12 & 4296 & 28.559 & 0.0230 & 3.71 & $60 \mathrm{C}$ Aqueous Phase SAW \\
\hline EWA 031 & Ti Grade 12 & 4296 & 28.310 & 0.0303 & 4.93 & $60 \mathrm{C}$ Water Line SAW \\
\hline EWD 001 & Ti Grade 12 & 4296 & 28.964 & 0.0688 & 10.93 & $60 \mathrm{C}$ Vapor Phase SAW \\
\hline EWD 002 & Ti Grade 12 & 4296 & 29.026 & 0.1822 & 28.89 & $60 \mathrm{C}$ Vapor Phase SAW \\
\hline EWD 003 & Ti Grade 12 & 4296 & 29.050 & 0.0433 & 6.86 & $60 \mathrm{C}$ Vapor Phase SAW \\
\hline EWD 004 & Ti Grade 12 & 4296 & 29.191 & 0.0123 & 1.94 & $60 \mathrm{C}$ Aqueous Phase SAW \\
\hline EWD 005 & Ti Grade 12 & 4296 & 28.990 & 0.0369 & 5.86 & $60 \mathrm{C}$ Aqueous Phase SAW \\
\hline EWD 006 & Ti Grade 12 & 4296 & 29.222 & 0.0384 & 6.05 & $60 \mathrm{C}$ Aqueous Phase SAW \\
\hline EWD 031 & Ti Grade 12 & 4296 & 29.249 & 0.1630 & 25.65 & $60 \mathrm{C}$ Water Line SAW \\
\hline FWA 001 & Ti Grade 16 & 4296 & 27.272 & 0.0188 & 3.11 & 60 C Vapor Phase SAW \\
\hline FWA 002 & $\pi$ Grade 16 & 4296 & 27.457 & 0.0222 & 3.65 & $60 \mathrm{C}$ Vapor Phase SAW \\
\hline FWA 003 & Ti Grade 16 & 4296 & 27.349 & 0.0259 & 4.27 & 60 C Vapor Phase SAW \\
\hline FWA 004 & $\pi$ Grade 16 & 4296 & 27.679 & -0.0001 & -0.02 & $60 \mathrm{C}$ Aqueous Phase SAW \\
\hline FWA 005 & Ti Grade 16 & 4296 & 27.246 & 0.0000 & 0.00 & $60 \mathrm{C}$ Aqueous Phase SAW \\
\hline FWA 006 & Ti Grade 16 & 4296 & 27.358 & -0.0002 & -0.03 & $60 \mathrm{C}$ Aqueous Phase SAW \\
\hline
\end{tabular}




\title{
Corrosion Rate Calculations for Weight Loss Specimens
}

For Rack Number : 25-1

\author{
Sample Allov Lag Time Surface Area Weight Corrosion Rate Test Environment \\ Identification (hours) (sq cm) Loss (g) (microns/year)
}

\begin{tabular}{|c|c|c|c|c|c|c|}
\hline FWA 031 & Ti Grade 16 & 4296 & 27.684 & 0.0001 & 0.02 & $60 \mathrm{C}$ Water Line SAW \\
\hline FWE 001 & Ti Grade 16 & 4296 & 27.419 & 0.0362 & 5.96 & $60 \mathrm{C}$ Vapor Phase SAW \\
\hline FWE 002 & Ti Grade 16 & 4296 & 27.624 & 00425 & 6.94 & $60 \mathrm{C}$ Vapor Phase SAW \\
\hline FWE 003 & Ti Grade 16 & 4296 & 28.128 & 0.0322 & 5.16 & $60 \mathrm{C}$ Vapor Phase SAW \\
\hline FWE 004 & Ti Grade 16 & 4296 & 28.100 & 0.0110 & 1.77 & $60 \mathrm{C}$ Aqueous Phase SAW \\
\hline FWE 005 & Ti Grade 16 & 4296 & 27.928 & 0.0099 & 1.60 & $60 \mathrm{C}$ Aqueous Phase SAW \\
\hline FWE 006 & Ti Grade 16 & 4296 & 28.013 & 0.0128 & 2.06 & $60 \mathrm{C}$ Aqueous Phase SAW \\
\hline FWE 031 & Ti Grade 16 & 4296 & 27.839 & 0.0008 & 0.13 & $60 \mathrm{C}$ Water Line SAW \\
\hline LWA 001 & Inconel 625 & 4296 & 28.217 & -00004 & -0.03 & $60 \mathrm{C}$ Vapor Phase SAW \\
\hline LWA 002 & Inconel 625 & 4296 & 28.258 & -0.0002 & -0.02 & $60 \mathrm{C}$ Vapor Phase SAW \\
\hline LWA 003 & Inconel 625 & 4296 & 28.143 & -0.0005 & -0.04 & $60 \mathrm{C}$ Vapor Phase SAW \\
\hline LWA 004 & Inconel 625 & 4296 & 28.291 & -0.0016 & -0.14 & $60 \mathrm{C}$ Aqueous Phase SAW \\
\hline LWA 005 & Inconel 625 & 4296 & 28.232 & -0.0017 & -0.15 & $60 \mathrm{C}$ Aqueous Phase SAW \\
\hline LWA 006 & Inconel 625 & 4296 & 28.180 & -0.0018 & -0.15 & $60 \mathrm{C}$ Aqueous Phase SAW \\
\hline LWA 031 & Inconel 625 & 4296 & 28.318 & -0.0005 & -0.04 & $60 \mathrm{C}$ Water Line SAW \\
\hline LWJ 001 & Inconel 625 & 4296 & 27.597 & -0.0004 & -0.04 & 60 C Vapor Phase SAW \\
\hline LWJ 002 & Inconel 625 & 4296 & 27.705 & -0.0012 & -0.10 & $60 \mathrm{C}$ Vapor Phase SAW \\
\hline LWJ 003 & Inconel 625 & 4296 & 27.722 & 0.0000 & 0.00 & $60 \mathrm{C}$ Vapor Phase SAW \\
\hline LWJ 004 & Inconel 625 & 4296 & 27.735 & -0.0001 & -0.01 & $60 \mathrm{C}$ Aqueous Phase SAW \\
\hline
\end{tabular}




\title{
Corrosion Rate Calculations for Weight Loss Specimens
} For Rack Number : 25-1

\author{
Sample Allov Lag Time Surface Area Weight Corrosion Rate Test Environment \\ Identification (hours) (sq cm) Loss (g) (microns/year)
}

\begin{tabular}{lllllll}
\hline LWJ 005 & Inconel 625 & 4296 & 27.679 & 0.0004 & 0.03 & 60 C Aqueous Phase SAW \\
LWJ 006 & Inconel 625 & 4296 & 27714 & -0.0004 & -0.03 & 60 C Aqueous Phase SAW \\
LWJ 031 & Inconel 625 & 4296 & 27.618 & -0.0004 & -0.03 & 60 C Water Line SAW
\end{tabular}




\section{Corrosion Rate Calculations for Weight Loss Specimens}

For Rack Number : 25-2

Sample Allov Lag Time Surface Area Weight Corrosion Rate Test Environment
$\begin{array}{llll}\text { Identification } & \text { (hours) } & \text { (sq cm) Loss (g) } & \text { (microns/year) }\end{array}$

\begin{tabular}{|c|c|c|c|c|c|c|}
\hline DWA 007 & $\mathrm{C} 22$ & 8376 & 28.302 & 0.0004 & 0.02 & $60 \mathrm{C}$ Vapor Phase SAW \\
\hline DWA 008 & $\mathrm{C} 22$ & 8376 & 28121 & 0.0003 & 0.01 & $60 \mathrm{C}$ Vapor Phase SAW \\
\hline DWA 009 & $\mathrm{C} 22$ & 8376 & 28.157 & 0.0006 & 0.03 & 60 C Vapor Phase SAW \\
\hline DWA 010 & C22 & 8376 & 27.990 & 0.0001 & 0.00 & $60 \mathrm{C}$ Aqueous Phase SAW \\
\hline DWA 011 & $\mathrm{C} 22$ & 8376 & 28.253 & 0.0004 & 0.02 & $60 \mathrm{C}$ Aqueous Phase SAW \\
\hline DWA 012 & $\mathrm{C} 22$ & 8376 & 28.041 & 0.0005 & 0.02 & $60 \mathrm{C}$ Aqueous Phase SAW \\
\hline DWA 032 & $\mathrm{C} 22$ & 8376 & 28.103 & 0.0003 & 0.01 & $60 \mathrm{C}$ Water Line SAW \\
\hline DWB 007 & $\mathrm{C} 22$ & 8376 & 27.503 & 0.0004 & 0.02 & $60 \mathrm{C}$ Vapor Phase SAW \\
\hline DWB 008 & $\mathrm{C} 22$ & 8376 & 27.316 & 0.0003 & 0.01 & $60 \mathrm{C}$ Vapor Phase SAW \\
\hline DWB 009 & $\mathrm{C} 22$ & 8376 & 27.466 & 0.0000 & 0.00 & $60 \mathrm{C}$ Vapor Phase SAW \\
\hline DWB 010 & $\mathrm{C} 22$ & 8376 & 27.337 & -0.0003 & -0.01 & $60 \mathrm{C}$ Aqueous Phase SAW \\
\hline DWB 011 & $\mathrm{C} 22$ & 8376 & 27.223 & -0.0002 & -0.01 & $60 \mathrm{C}$ Aqueous Phase SAW \\
\hline DWB 012 & $\mathrm{C} 22$ & 8376 & 27.312 & -0.0001 & 0.00 & $60 \mathrm{C}$ Aqueous Phase SAW \\
\hline DWB 032 & $\mathrm{C} 22$ & 8376 & 27.597 & 0.0002 & 0.01 & 60 C Water Line SAW \\
\hline LWA 007 & Inconel 625 & 8376 & 28.245 & 0.0000 & 0.00 & $60 \mathrm{C}$ Vapor Phase SAW \\
\hline LWA 008 & Inconel 625 & 8376 & 28.267 & -0.0004 & -0.02 & $60 \mathrm{C}$ Vapor Phase SAW \\
\hline LWA 009 & Inconel 625 & 8376 & 28.274 & -0.0002 & -0.01 & 60 C Vapor Phase SAW \\
\hline LWA 010 & Inconel 625 & 8376 & 28.165 & -0.0004 & -0.02 & $60 \mathrm{C}$ Aqueous Phase SAW \\
\hline LWA 011 & Inconel 625 & 8376 & 28.206 & -0.0004 & -0.02 & $60 \mathrm{C}$ Aqueous Phase SAW \\
\hline
\end{tabular}




\begin{tabular}{|c|c|c|c|c|c|c|}
\hline $\begin{array}{l}\text { Sample } \\
\text { Identification }\end{array}$ & Allov & $\begin{array}{r}\text { Lag Time } \\
\text { (hours) }\end{array}$ & $\begin{array}{r}\text { Surface Area } \\
(s q \mathrm{~cm})\end{array}$ & $\begin{array}{l}\text { Weight } \\
\text { Loss (g) }\end{array}$ & $\begin{array}{l}\text { Corrosion Rate } \\
\text { (microns/year) }\end{array}$ & Test Environment \\
\hline LWA 012 & Inconel 625 & 8376 & 28.196 & -0.0006 & -0.03 & $60 \mathrm{C}$ Aqueous Phase SAW \\
\hline LWA 032 & Inconel 625 & 8376 & 28374 & -0.0001 & 0.00 & $60 \mathrm{C}$ Water Line SAW \\
\hline LWJ 007 & Inconel 625 & 8376 & 27788 & 0.0000 & 0.00 & 60 C Vapor Phase SAW \\
\hline LWJ 008 & Inconel 625 & 8376 & 27.700 & 0.0000 & 0.00 & 60 C Vapor Phase SAW \\
\hline LWJ 009 & Inconel 625 & 8376 & 27.773 & -0.0001 & 0.00 & 60 C Vapor Phase SAW \\
\hline LWJ 010 & Inconel 625 & 8376 & 27.620 & 0.0001 & 0.00 & $60 \mathrm{C}$ Aqueous Phase SAW \\
\hline LWJ 011 & Inconel 625 & 8376 & 27.759 & 0.0002 & 0.01 & $60 \mathrm{C}$ Aqueous Phase SAW \\
\hline LWJ 012 & Inconel 625 & 8376 & 27.738 & -0.0002 & -0.01 & $60 \mathrm{C}$ Aqueous Phase SAW \\
\hline LWJ 032 & Inconel 625 & 8376 & 27.536 & -0.0002 & -0.01 & $60 \mathrm{C}$ Water Line SAW \\
\hline
\end{tabular}




\section{Corrosion Rate Calculations for Weight Loss Specimens For Rack Number : 26-1}

Sample Allov Lag Time Surface Area Weight Corrosion Rate Test Environment Identification (hours) (sq cm) Loss (g) (microns/year)

\begin{tabular}{|c|c|c|c|c|c|c|}
\hline AWA 036 & 1825 & 4344 & 27.942 & 00005 & 0.04 & $90 \mathrm{C}$ Water Line SAW \\
\hline AWA 041 & 1825 & 4344 & 28.294 & 0.0011 & 0.10 & $90 \mathrm{C}$ Vapor Phase SAW \\
\hline AWA 042 & 1825 & 4344 & 28.243 & 0.0016 & 0.14 & $90 \mathrm{C}$ Vapor Phase SAW \\
\hline AWA 043 & 1825 & 4344 & 28.118 & 0.0008 & 0.07 & $90 \mathrm{C}$ Vapor Phase SAW \\
\hline AWA 044 & 1825 & 4344 & 28.440 & 0.0019 & 0.17 & $90 \mathrm{C}$ Aqueous Phase SAW \\
\hline AWA 045 & 1825 & 4344 & 28.038 & 0.0013 & 0.11 & $90 \mathrm{C}$ Aqueous Phase SAW \\
\hline AWA 046 & 1825 & 4344 & 28.353 & 0.0018 & 0.16 & $90 \mathrm{C}$ Aqueous Phase SAW \\
\hline AWB 036 & 1825 & 4344 & 27.453 & 00006 & 0.05 & 90 C Water Line SAW \\
\hline AWB 041 & 1825 & 4344 & 27.828 & 0.0009 & 0.08 & $90 \mathrm{C}$ Vapor Phase SAW \\
\hline AWB 042 & 1825 & 4344 & 27.683 & 00006 & 0.05 & $90 \mathrm{C}$ Vapor Phase SAW \\
\hline AWB 043 & 1825 & 4344 & 27.494 & 0.0007 & 0.06 & $90 \mathrm{C}$ Vapor Phase SAW \\
\hline AWB 044 & 1825 & 4344 & 27.477 & 0.0055 & 0.50 & $90 \mathrm{C}$ Aqueous Phase SAW \\
\hline AWB 045 & 1825 & 4344 & 27.721 & 0.0016 & 0.14 & $90 \mathrm{C}$ Aqueous Phase SAW \\
\hline AWB 046 & 1825 & 4344 & 27.638 & 0.0040 & 0.36 & $90 \mathrm{C}$ Aqueous Phase SAW \\
\hline BWA 036 & G3 & 4344 & 28.607 & 0.0002 & 0.02 & $90 \mathrm{C}$ Water Line SAW \\
\hline BWA 041 & G3 & 4344 & 28.764 & 0.0000 & 0.00 & 90 C Vapor Phase SAW \\
\hline BWA 042 & G3 & 4344 & 28.313 & 0.0003 & 0.03 & 90 C Vapor Phase SAW \\
\hline BWA 043 & G3 & 4344 & 28.351 & 0.0000 & 0.00 & $90 \mathrm{C}$ Vapor Phase SAW \\
\hline BWA 044 & G3 & 4344 & 28.605 & -0.0004 & -0.03 & $90 \mathrm{C}$ Aqueous Phase SAW \\
\hline
\end{tabular}




\section{Corrosion Rate Calculations for Weight Loss Specimens}

For Rack Number : 26-1

Sample Allov Lag Time Surface Area Weight Corrosion Rate Test Environment Identification (hours) (sq cm) Loss (g) (microns/year)

\begin{tabular}{|c|c|c|c|c|c|c|}
\hline BWA 045 & G3 & 4344 & 28.409 & 00000 & 0.00 & $90 \mathrm{C}$ Aqueous Phase SAW \\
\hline BWA 046 & G3 & 4344 & 28.475 & -0.0003 & -0.03 & $90 \mathrm{C}$ Aqueous Phase SAW \\
\hline BWC 036 & G3 & 4344 & 28.341 & 0.0009 & 0.08 & 90 C Water Line SAW \\
\hline BWC 041 & G3 & 4344 & 28.249 & 0.0008 & 0.07 & $90 \mathrm{C}$ Vapor Phase SAW \\
\hline BWC 042 & G3 & 4344 & 28.302 & 0.0003 & 0.03 & $90 \mathrm{C}$ Vapor Phase SAW \\
\hline BWC 043 & G3 & 4344 & 27.851 & 0.0003 & 0.03 & 90 C Vapor Phase SAW \\
\hline BWC 044 & G3 & 4344 & 28.321 & -0.0005 & -0.04 & $90 \mathrm{C}$ Aqueous Phase SAW \\
\hline BWC 045 & G3 & 4344 & 28.312 & 0.0011 & 0.09 & $90 \mathrm{C}$ Aqueous Phase SAW \\
\hline BWC 046 & G3 & 4344 & 28.327 & -0.0001 & -0.01 & $90 \mathrm{C}$ Aqueous Phase SAW \\
\hline CWA 036 & C4 & 4344 & 27.812 & 0.0004 & 0.03 & $90 \mathrm{C}$ Water Line SAW \\
\hline CWA 041 & $\mathrm{C} 4$ & 4344 & 28.001 & -0.0001 & -0.01 & $90 \mathrm{C}$ Vapor Phase SAW \\
\hline CWA 042 & $\mathrm{C} 4$ & 4344 & 27.863 & 0.0008 & 0.07 & 90 C Vapor Phase SAW \\
\hline CWA 043 & $\mathrm{C} 4$ & 4344 & 27.938 & 0.0004 & 0.03 & 90 C Vapor Phase SAW \\
\hline CWA 044 & C4 & 4344 & 27.982 & 0.0006 & 0.05 & $90 \mathrm{C}$ Aqueous Phase SAW \\
\hline CWA 045 & $\mathrm{C} 4$ & 4344 & 28.203 & 0.0008 & 0.07 & $90 \mathrm{C}$ Aqueous Phase SAW \\
\hline CWA 046 & $\mathrm{C4}$ & 4344 & 28.066 & 0.0007 & 0.06 & $90 \mathrm{C}$ Aqueous Phase SAW \\
\hline CWB 036 & $\mathrm{C}_{4}$ & 4344 & 26.918 & 0.0010 & 0.09 & $90 \mathrm{C}$ Water Line SAW \\
\hline CWB 041 & $\mathrm{C}_{4}$ & 4344 & 27.638 & 0.0006 & 0.05 & $90 \mathrm{C}$ Vapor Phase SAW \\
\hline CWB 042 & $\mathbf{C 4}$ & 4344 & 27.851 & 0.0006 & 0.05 & 90 C Vapor Phase SAW \\
\hline
\end{tabular}




\section{Corrosion Rate Calculations for Weight Loss Specimens}

For Rack Number : 26-1

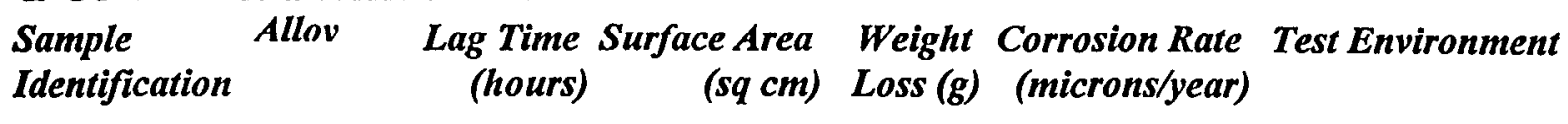

\begin{tabular}{|c|c|c|c|c|c|c|}
\hline CWB 043 & $\mathrm{C4}$ & 4344 & 27.153 & 0.0008 & 0.07 & $90 \mathrm{C}$ Vapor Phase SAW \\
\hline CWB 044 & $\mathrm{C4}$ & 4344 & 27.773 & 0.0004 & 0.03 & $90 \mathrm{C}$ Aqueous Phase SAW \\
\hline CWB 045 & $\mathrm{C} 4$ & 4344 & 27.763 & 0.0002 & 0.02 & $90 \mathrm{C}$ Aqueous Phase SAW \\
\hline CWB 046 & $\mathrm{C} 4$ & 4344 & 27.896 & 0.0002 & 0.02 & $90 \mathrm{C}$ Aqueous Phase SAW \\
\hline DWA 036 & $\mathrm{C} 22$ & 4344 & 27.887 & 0.0002 & 0.02 & $90 \mathrm{C}$ Water Line SAW \\
\hline DWA 041 & $\mathrm{C} 22$ & 4344 & 28.137 & 0.0000 & 0.00 & $90 \mathrm{C}$ Vapor Phase SAW \\
\hline DWA 042 & $\mathrm{C} 22$ & 4344 & 28.111 & 0.0006 & 0.05 & 90 C Vapor Phase SAW \\
\hline DWA 043 & $\mathrm{C} 22$ & 4344 & 28.085 & 0.0010 & 0.08 & $90 \mathrm{C}$ Vapor Phase SAW \\
\hline DWA 044 & $\mathrm{C} 22$ & 4344 & 28.110 & -0.0004 & -0.03 & $90 \mathrm{C}$ Aqueous Phase SAW \\
\hline DWA 045 & $\mathrm{C} 22$ & 4344 & 28.074 & -0.0005 & -0.04 & $90 \mathrm{C}$ Aqueous Phase SAW \\
\hline DWA 046 & $\mathrm{C} 22$ & 4344 & 28.085 & 0.0001 & 0.01 & $90 \mathrm{C}$ Aqueous Phase SAW \\
\hline DWB 036 & $\mathrm{C} 22$ & 4344 & 27.547 & 0.0001 & 0.01 & $90 \mathrm{C}$ Water Line SAW \\
\hline DWB 041 & $\mathrm{C} 22$ & 4344 & 27.366 & 0.0005 & 0.04 & 90 C Vapor Phase SAW \\
\hline DWB 042 & $\mathrm{C} 22$ & 4344 & 27.132 & 0.0014 & 0.12 & $90 \mathrm{C}$ Vapor Phase SAW \\
\hline DWB 043 & $\mathrm{C} 22$ & 4344 & 27.639 & 0.0002 & 0.02 & $90 \mathrm{C}$ Vapor Phase SAW \\
\hline DWB 044 & $\mathrm{C} 22$ & 4344 & 27.347 & -0.0001 & -0.01 & $90 \mathrm{C}$ Aqueous Phase SAW \\
\hline DWB 045 & $\mathrm{C} 22$ & 4344 & 27.120 & -0.0001 & -0.01 & $90 \mathrm{C}$ Aqueous Phase SAW \\
\hline DWB 046 & $\mathrm{C} 22$ & 4344 & 26.866 & -0.0002 & -0.02 & $90 \mathrm{C}$ Aqueous Phase SAW \\
\hline EWA 036 & Ti Grade 12 & 4344 & 27.841 & 0.0158 & 2.58 & $90 \mathrm{C}$ Water Line SAW \\
\hline
\end{tabular}




\section{Corrosion Rate Calculations for Weight Loss Specimens For Rack Number : 26-1}

Sample Allov Lag Time Surface Area
$\begin{aligned} & \text { (hours) } \\ & \text { Identification }\end{aligned} \quad(\mathrm{sq} \mathrm{cm})$

\begin{tabular}{|c|c|c|c|c|c|c|}
\hline EWA 041 & Ti Grade 12 & 4344 & 28.050 & 0.0551 & 8.94 & $90 \mathrm{C}$ Vapor Phase SAW \\
\hline EWA 042 & Ti Grade 12 & 4344 & 28.398 & 0.0212 & 3.40 & $90 \mathrm{C}$ Vapor Phase SAW \\
\hline EWA 043 & Ti Grade 12 & 4344 & 28.609 & 0.0550 & 8.75 & 90 C Vapor Phase SAW \\
\hline EWA 044 & Ti Grade 12 & 4344 & 28.762 & 0.0270 & 4.27 & $90 \mathrm{C}$ Aqueous Phase SAW \\
\hline EWA 045 & Ti Grade 12 & 4344 & 29.079 & 0.0357 & 5.59 & $90 \mathrm{C}$ Aqueous Phase SAW \\
\hline EWA 046 & Ti Grade 12 & 4344 & 28.432 & 0.0150 & 2.40 & $90 \mathrm{C}$ Aqueous Phase SAW \\
\hline EWD 036 & Ti Grade 12 & 4344 & 29.093 & 0.0192 & 3.00 & $90 \mathrm{C}$ Water Line SAW \\
\hline EWD 041 & Ti Grade 12 & 4344 & 29.240 & 0.0211 & 3.28 & $90 \mathrm{C}$ Vapor Phase SAW \\
\hline EWD 042 & Ti Grade 12 & 4344 & 29.158 & 0.0678 & 10.58 & $90 \mathrm{C}$ Vapor Phase SAW \\
\hline EWD 0.43 & Ti Grade 12 & 4344 & 29.218 & 0.0468 & 7.29 & 90 C Vapor Phase SAW \\
\hline EWD 044 & Ti Grade 12 & 4344 & 29.226 & 0.0237 & 3.69 & $90 \mathrm{C}$ Aqueous Phase SAW \\
\hline EWD 045 & Ti Grade 12 & 4344 & 29.217 & 0.0178 & 2.77 & $90 \mathrm{C}$ Aqueous Phase SAW \\
\hline EWD 046 & Ti Grade 12 & 4344 & 29.099 & 0.0240 & 3.75 & $90 \mathrm{C}$ Aqueous Phase SAW \\
\hline FWA 036 & Ti Grade 16 & 4344 & 27.651 & 0.0166 & 2.68 & $90 \mathrm{C}$ Water Line SAW \\
\hline FWA 041 & TI Grade 16 & 4344 & 27.583 & 0.0198 & 3.20 & $90 \mathrm{C}$ Vapor Phase SAW \\
\hline FWA 042 & Ti Grade 16 & 4344 & 27.501 & 0.0187 & 3.03 & $90 \mathrm{C}$ Vapor Phase SAW \\
\hline FWA 043 & Ti Grade 16 & 4344 & 27.616 & 0.0223 & 3.60 & 90 C Vapor Phase SAW \\
\hline FWA 044 & Ti Grade 16 & 4344 & 27.485 & 0.0143 & 2.32 & $90 \mathrm{C}$ Aqueous Phase SAW \\
\hline FWA 045 & Ti Grade 16 & 4344 & 27.470 & 0.0148 & 2.40 & $90 \mathrm{C}$ Aqueous Phase SAW \\
\hline
\end{tabular}




\section{Corrosion Rate Calculations for Weight Loss Specimens For Rack Number : 26-1}

Sample Allov Lag Time Surface Area Weight Corrosion Rate Test Environment Identification (hours) (sq cm) Loss (g) (microns/year)

\begin{tabular}{|c|c|c|c|c|c|c|}
\hline FWA 046 & Ti Grade 16 & 4344 & 27.585 & 0.0127 & 2.05 & $90 \mathrm{C}$ Aqueous Phase SAW \\
\hline FWE 036 & Ti Grade 16 & 4344 & 28.059 & 0.0181 & 288 & $90 \mathrm{C}$ Water Line SAW \\
\hline FWE 041 & Ti Grade 16 & 4344 & 27.888 & 0.0212 & 3.39 & $90 \mathrm{C}$ Vapor Phase SAW \\
\hline FWE 042 & Ti Grade 16 & 4344 & 27.736 & 0.0331 & 5.32 & 90 C Vapor Phase SAW \\
\hline FWE 043 & Ti Grade 16 & 4344 & 27.988 & 0.0204 & 3.25 & 90 C Vapor Phase SAW \\
\hline FWE 045 & Ti Grade 16 & 4344 & 28.007 & 0.0110 & 1.75 & $90 \mathrm{C}$ Aqueous Phase SAW \\
\hline FWE 046 & Ti Grade 16 & 4344 & 27.785 & 0.0111 & 1.78 & $90 \mathrm{C}$ Aqueous Phase SAW \\
\hline LWA 036 & Inconel 625 & 4344 & 27.813 & 0.0004 & 0.03 & $90 \mathrm{C}$ Water Line SAW \\
\hline LWA 041 & Inconel 625 & 4344 & 28.317 & 0.0002 & 0.02 & 90 C Vapor Phase SAW \\
\hline LWA 042 & Inconel 625 & 4344 & 28.433 & 0.0001 & 0.01 & $90 \mathrm{C}$ Vapor Phase SAW \\
\hline LWA 043 & Inconel 625 & 4344 & 28.371 & 0.0005 & 0.04 & $90 \mathrm{C}$ Vapor Phase SAW \\
\hline LWA 044 & Inconel 625 & 4344 & 28.301 & -0.0004 & -0.03 & $90 \mathrm{C}$ Aqueous Phase SAW \\
\hline LWA 045 & Inconel 625 & 4344 & 28.237 & -0.0010 & -0.08 & $90 \mathrm{C}$ Aqueous Phase SAW \\
\hline LWA 046 & Inconel 625 & 4344 & 28.201 & -0.0006 & -0.05 & $90 \mathrm{C}$ Aqueous Phase SAW \\
\hline LWJ 036 & Inconel 625 & 4344 & 27.583 & 0.0002 & 0.02 & $90 \mathrm{C}$ Water Line SAW \\
\hline LWJ 041 & Inconel 625 & 4344 & 27.468 & 0.0001 & 0.01 & 90 C Vapor Phase SAW \\
\hline LWJ 042 & Inconel 625 & 4344 & 27.657 & 0.0001 & 0.01 & 90 C Vapor Phase SAW \\
\hline LWJ 043 & Inconel 625 & 4344 & 27.767 & 0.0012 & 0.10 & 90 C Vapor Phase SAW \\
\hline LWJ 044 & Inconel 625 & 4344 & 27.683 & 0.0011 & 0.09 & $90 \mathrm{C}$ Aqueous Phase SAW \\
\hline
\end{tabular}




\section{Corrosion Rate Calculations for Weight Loss Specimens}

For Rack Number : 26-1

\begin{tabular}{|c|c|c|c|c|c|c|}
\hline $\begin{array}{l}\text { Sample } \\
\text { Identification }\end{array}$ & Allov & $\begin{array}{r}\text { Lag Time } \\
\text { (hours) }\end{array}$ & $\begin{array}{r}\text { Surface Area } \\
(s q \mathrm{~cm})\end{array}$ & $\begin{array}{l}\text { Weight } \\
\text { Loss }(g)\end{array}$ & $\begin{array}{l}\text { Corrosion Rate } \\
\text { (microns/year) }\end{array}$ & Test Environment \\
\hline LWJ 045 & Inconel 625 & 4344 & 27.785 & -00005 & -0.04 & $90 \mathrm{C}$ Aqueous Phase SAW \\
\hline LWJ 046 & Inconel 625 & 4344 & 27.672 & 0.0001 & 0.01 & $90 \mathrm{C}$ Aqueous Phase SAW \\
\hline
\end{tabular}




\section{Corrosion Rate Calculations for Weight Loss Specimens}

For Rack Number : 27-1

Sample Allov Lag Time Surface Area Weight Corrosion Rate Test Environment Identification (hours) (sq cm) Loss (g) (microns/year)

\begin{tabular}{|c|c|c|c|c|c|c|}
\hline AWA 071 & 1825 & 4392 & 28.340 & -0.0001 & -0.01 & $60 \mathrm{C}$ Vapor Phase SCW \\
\hline AWA 072 & 1825 & 4392 & 28.131 & 0.0004 & 0.03 & $60 \mathrm{C}$ Vapor Phase SCW \\
\hline AWA 073 & 1825 & 4392 & 28.300 & 0.0003 & 0.03 & $60 \mathrm{C}$ Vapor Phase SCW \\
\hline AWA 074 & 1825 & 4392 & 28.162 & 0.0007 & 0.06 & $60 \mathrm{C}$ Aqueous Phase SCW \\
\hline AWA 075 & 1825 & 4392 & 28.179 & 0.0006 & 0.05 & $60 \mathrm{C}$ Aqueous Phase SCW \\
\hline AWA 076 & 1825 & 4392 & 28.198 & 0.0006 & 0.05 & $60 \mathrm{C}$ Aqueous Phase SCW \\
\hline AWA 101 & 1825 & 4392 & 28.141 & 0.0006 & 0.05 & $60 \mathrm{C}$ Water Line SCW \\
\hline AWB 071 & 1825 & 4392 & 27.628 & 0.0002 & 0.02 & $60 \mathrm{C}$ Vapor Phase SCW \\
\hline AWB 072 & 1825 & 4392 & 27.828 & 0.0010 & 0.09 & $60 \mathrm{C}$ Vapor Phase SCW \\
\hline AWB 073 & 1825 & 4392 & 27.750 & 0.0005 & 0.04 & $60 \mathrm{C}$ Vapor Phase SCW \\
\hline AWB 074 & 1825 & 4392 & 27.896 & 0.0003 & 0.03 & $60 \mathrm{C}$ Aqueous Phase SCW \\
\hline AWB 075 & 1825 & 4392 & 27.373 & 0.0007 & 0.06 & $60 \mathrm{C}$ Aqueous Phase SCW \\
\hline AWB 076 & 1825 & 4392 & 27.255 & 0.0001 & 0.01 & $60 \mathrm{C}$ Aqueous Phase SCW \\
\hline AWB 101 & 1825 & 4392 & 27.545 & 0.0007 & 0.06 & $60 \mathrm{C}$ Water Line SCW \\
\hline BWA 071 & G3 & 4392 & 28.560 & 0.0001 & 0.01 & 60 C Vapor Phase SCW \\
\hline BWA 072 & G3 & 4392 & 28.548 & 0.0006 & 0.05 & $60 \mathrm{C}$ Vapor Phase SCW \\
\hline BWA 073 & G3 & 4392 & 28.445 & 0.0002 & 0.02 & 60 C Vapor Phase SCW \\
\hline BWA 074 & G3 & 4392 & 28.696 & 0.0003 & 0.03 & $60 \mathrm{C}$ Aqueous Phase SCW \\
\hline BWA 075 & G3 & 4392 & 28.718 & 0.0008 & 0.07 & $60 \mathrm{C}$ Aqueous Phase SCW \\
\hline
\end{tabular}




\section{Corrosion Rate Calculations for Weight Loss Specimens}

For Rack Number : 27-1

$\begin{array}{llrl}\text { Sample Allov Lag Time Surface Area Weight Corrosion Rate Test Environment } \\ \text { Identification } & \text { (hours) } & \text { (sq cm) } & \text { Loss (g) (microns/year) }\end{array}$

\begin{tabular}{|c|c|c|c|c|c|c|}
\hline BWA 076 & G3 & 4392 & 28.782 & 0.0002 & 0.02 & $60 \mathrm{C}$ Aqueous Phase SCW \\
\hline BWA 101 & G3 & 4392 & 28.415 & 0.0005 & 0.04 & $60 \mathrm{C}$ Water Line SCW \\
\hline BWC 071 & G3 & 4392 & 28.315 & 0.0005 & 0.04 & $60 \mathrm{C}$ Vapor Phase SCW \\
\hline BWC 072 & G3 & 4392 & 27.887 & 0.0001 & 0.01 & $60 \mathrm{C}$ Vapor Phase SCW \\
\hline BWC 073 & G3 & 4392 & 28.265 & 0.0021 & 0.18 & $60 \mathrm{C}$ Vapor Phase SCW \\
\hline BWC 074 & G3 & 4392 & 28.410 & 0.0006 & 0.05 & $60 \mathrm{C}$ Aqueous Phase SCW \\
\hline BWC 075 & G3 & 4392 & 28.510 & 0.0000 & 0.00 & $60 \mathrm{C}$ Aqueous Phase SCW \\
\hline BWC 076 & G3 & 4392 & 28.281 & 0.0002 & 0.02 & $60 \mathrm{C}$ Aqueous Phase SCW \\
\hline BWC 101 & G3 & 4392 & 27.685 & 0.0005 & 0.04 & $60 \mathrm{C}$ Water Line SCW \\
\hline CWA 071 & $\mathrm{C4}$ & 4392 & 27.830 & 0.0005 & 0.04 & $60 \mathrm{C}$ Vapor Phase SCW \\
\hline CWA 072 & $\mathrm{C4}$ & 4392 & 28.400 & 0.0002 & 0.02 & 60 C Vapor Phase SCW \\
\hline CWA 073 & $\mathrm{C4}$ & 4392 & 27.620 & 0.0007 & 0.06 & $60 \mathrm{C}$ Vapor Phase SCW \\
\hline CWA 074 & $\mathrm{C4}$ & 4392 & 28.069 & 0.0008 & 0.07 & $60 \mathrm{C}$ Aqueous Phase SCW \\
\hline CWA 075 & $\mathrm{C4}$ & 4392 & 27.723 & 0.0011 & 0.09 & $60 \mathrm{C}$ Aqueous Phase SCW \\
\hline CWA 076 & $\mathrm{C4}$ & 4392 & 28.230 & 0.0008 & 0.07 & $60 \mathrm{C}$ Aqueous Phase SCW \\
\hline CWA 101 & $\mathrm{C4}$ & 4392 & 28.325 & 0.0005 & 0.04 & $60 \mathrm{C}$ Water Line SCW \\
\hline CWB 071 & $\mathrm{C4}$ & 4392 & 28.070 & 0.0005 & 0.04 & $60 \mathrm{C}$ Vapor Phase SCW \\
\hline CWB 072 & $\mathrm{C4}$ & 4392 & 27.835 & 0.0010 & 0.08 & $60 \mathrm{C}$ Vapor Phase SCW \\
\hline CWB 073 & $\mathrm{C4}$ & 4392 & 27.847 & 0.0007 & 0.06 & $60 \mathrm{C}$ Vapor Phase SCW \\
\hline
\end{tabular}




\section{Corrosion Rate Calculations for Weight Loss Specimens}

For Rack Number : 27-1

Sample Allov Lag Time Surface Area Weight Corrosion Rate Test Environment Identification (hours) (sq cm) Loss (g) (microns/year)

\begin{tabular}{|c|c|c|c|c|c|c|}
\hline CWB 074 & $\mathrm{C4}$ & 4392 & 27.829 & 0.0007 & 0.06 & $60 \mathrm{C}$ Aqueous Phase SCW \\
\hline CWB 075 & $\mathrm{C} 4$ & 4392 & 27.675 & 0.0010 & 0.08 & $60 \mathrm{C}$ Aqueous Phase SCW \\
\hline CWB 076 & $\mathrm{C} 4$ & 4392 & 27.351 & 0.0014 & 0.12 & $60 \mathrm{C}$ Aqueous Phase SCW \\
\hline CWB 101 & $\mathrm{C} 4$ & 4392 & 27.444 & 0.0012 & 0.10 & $60 \mathrm{C}$ Water Line SCW \\
\hline DWA 071 & $\mathrm{C} 22$ & 4392 & 28.197 & 0.0003 & 0.02 & $60 \mathrm{C}$ Vapor Phase SCW \\
\hline DWA 072 & $\mathrm{C} 22$ & 4392 & 28.183 & 0.0004 & 0.03 & $60 \mathrm{C}$ Vapor Phase SCW \\
\hline DWA 073 & $\mathrm{C} 22$ & 4392 & 28.055 & 0.0010 & 0.08 & $60 \mathrm{C}$ Vapor Phase SCW \\
\hline DWA 074 & $\mathrm{C} 22$ & 4392 & 28.119 & 0.0011 & 0.09 & $60 \mathrm{C}$ Aqueous Phase SCW \\
\hline DWA 075 & $\mathrm{C} 22$ & 4392 & 28.136 & 0.0009 & 0.07 & $60 \mathrm{C}$ Aqueous Phase SCW \\
\hline DWA 076 & $\mathrm{C} 22$ & 4392 & 28.291 & 0.0005 & 0.04 & $60 \mathrm{C}$ Aqueous Phase SCW \\
\hline DWA 101 & $\mathrm{C} 22$ & 4392 & 28.366 & 0.0006 & 0.05 & $60 \mathrm{C}$ Water Line SCW \\
\hline DWB 071 & $\mathrm{C} 22$ & 4392 & 27.472 & 0.0003 & 0.03 & $60 \mathrm{C}$ Vapor Phase SCW \\
\hline DWB 072 & C22 & 4392 & 27.611 & 0.0003 & 0.03 & 60 C Vapor Phase SCW \\
\hline DWB 073 & C22 & 4392 & 27.479 & 0.0004 & 0.03 & 60 C Vapor Phase SCW \\
\hline DWB 074 & $\mathrm{C} 22$ & 4392 & 27.441 & 0.0005 & 0.04 & $60 \mathrm{C}$ Aqueous Phase SCW \\
\hline DWB 075 & $\mathrm{C} 22$ & 4392 & 27.568 & 0.0004 & 0.03 & $60 \mathrm{C}$ Aqueous Phase SCW \\
\hline DWB 076 & $\mathrm{C} 22$ & 4392 & 27.434 & 0.0008 & 0.07 & $60 \mathrm{C}$ Aqueous Phase SCW \\
\hline DWB 101 & $\mathrm{C} 22$ & 4392 & 27.460 & 0.0008 & 0.07 & $60 \mathrm{C}$ Water Line SCW \\
\hline EWA 071 & Ti Grade 12 & 4392 & 28.320 & 0.0404 & 6.42 & $60 \mathrm{C}$ Vapor Phase SCW \\
\hline
\end{tabular}




\section{Corrosion Rate Calculations for Weight Loss Specimens For Rack Number : 27-1}

Sample Allov Lag Time Surface Area Weight Corrosion Rate Test Environment Identification (hours) (sq cm) Loss (g) (microns/year)

\begin{tabular}{|c|c|c|c|c|c|c|}
\hline EWA 072 & TI Grade 12 & 4392 & 28.507 & 0.0527 & 8.32 & 60 C Vapor Phase SCW \\
\hline EWA 073 & Ti Grade 12 & 4392 & 28598 & 0.0539 & 8.49 & 60 C Vapor Phase SCW \\
\hline EWA 074 & Ti Grade 12 & 4392 & 28.144 & 0.0532 & 8.51 & $60 \mathrm{C}$ Aqueous Phase SCW \\
\hline EWA 075 & Ti Grade 12 & 4392 & 28.498 & 0.0440 & 6.95 & $60 \mathrm{C}$ Aqueous Phase SCW \\
\hline EWA 076 & Ti Grade 12 & 4392 & 28.628 & 0.0470 & 7.39 & $60 \mathrm{C}$ Aqueous Phase SCW \\
\hline EWA 101 & Ti Grade 12 & 4392 & 29.065 & 0.0491 & 7.61 & 60 C Water Line SCW \\
\hline EWD 071 & Ti Grade 12 & 4392 & 28.951 & 0.0434 & 6.75 & 60 C Vapor Phase SCW \\
\hline EWD 072 & Ti Grade 12 & 4392 & 29.134 & 0.0370 & 5.72 & $60 \mathrm{C}$ Vapor Phase SCW \\
\hline EWD 073 & Ti Grade 12 & 4392 & 29.187 & 0.0428 & 6.60 & $60 \mathrm{C}$ Vapor Phase SCW \\
\hline EWD 074 & Ti Grade 12 & 4392 & 29.085 & 0.0325 & 5.03 & $60 \mathrm{C}$ Aqueous Phase SCW \\
\hline EWD 075 & Ti Grade 12 & 4392 & 29.123 & 0.0489 & 7.56 & $60 \mathrm{C}$ Aqueous Phase SCW \\
\hline EWD 076 & Ti Grade 12 & 4392 & 28.972 & 0.0371 & 5.77 & $60 \mathrm{C}$ Aqueous Phase SCW \\
\hline EWD 101 & Ti Grade 12 & 4392 & 29.117 & 0.0445 & 6.88 & $60 \mathrm{C}$ Water Line SCW \\
\hline FWA 071 & Ti Grade 16 & 4392 & 27.701 & 0.0177 & 2.82 & 60 C Vapor Phase SCW \\
\hline FWA 072 & Ti Grade 16 & 4392 & 27.373 & 0.0310 & 5.00 & 60 C Vapor Phase SCW \\
\hline FWA 073 & Ti Grade 16 & 4392 & 27.510 & 0.0196 & 3.14 & $60 \mathrm{C}$ Vapor Phase SCW \\
\hline FWA 074 & TI Grade 16 & 4392 & 27.770 & 0.0214 & 3.40 & $60 \mathrm{C}$ Aqueous Phase SCW \\
\hline FWA 075 & Ti Grade 16 & 4392 & 27.678 & 0.0147 & 2.34 & $60 \mathrm{C}$ Aqueous Phase SCW \\
\hline FWA 076 & Ti Grade 16 & 4392 & 26.735 & 0.0181 & 2.99 & $60 \mathrm{C}$ Aqueous Phase SCW \\
\hline
\end{tabular}




\section{Corrosion Rate Calculations for Weight Loss Specimens For Rack Number : 27-1}

Sample Allov Lag Time Surface Area Weight Corrosion Rate Test Environment Identification (hours) (sq cm) Loss (g) (microns/year)

\begin{tabular}{|c|c|c|c|c|c|c|}
\hline FWA 101 & Ti Grade 16 & 4392 & 27.760 & 0.0238 & 3.78 & $60 \mathrm{C}$ Water Line SCW \\
\hline FWE 071 & Ti Grade 16 & 4392 & 27.726 & 0.0201 & 3.20 & $60 \mathrm{C}$ Vapor Phase SCW \\
\hline FWE 072 & Ti Grade 16 & 4392 & 27.908 & 0.0216 & 3.42 & 60 C Vapor Phase SCW \\
\hline FWE 073 & Ti Grade 16 & 4392 & 27.659 & 0.0311 & 4.96 & $60 \mathrm{C}$ Vapor Phase SCW \\
\hline FWE 074 & Ti Grade 16 & 4392 & 28.150 & 0.0240 & 3.76 & $60 \mathrm{C}$ Aqueous Phase SCW \\
\hline FWE 075 & Ti Grade 16 & 4392 & 27.989 & 0.0214 & 3.37 & $60 \mathrm{C}$ Aqueous Phase SCW \\
\hline FWE 076 & Ti Grade 16 & 4392 & 27.510 & 0.0187 & 3.00 & $60 \mathrm{C}$ Aqueous Phase SCW \\
\hline FWE 101 & Ti Grade 16 & 4392 & 27.721 & 0.0299 & 4.76 & $60 \mathrm{C}$ Water Line SCW \\
\hline LWA 071 & Inconel 625 & 4392 & 28.266 & 00011 & 0.09 & $60 \mathrm{C}$ Vapor Phase SCW \\
\hline LWA 072 & Inconel 625 & 4392 & 28.240 & 0.0008 & 0.07 & $60 \mathrm{C}$ Vapor Phase SCW \\
\hline LWA 073 & Inconel 625 & 4392 & 28250 & 0.0010 & 0.08 & $60 \mathrm{C}$ Vapor Phase SCW \\
\hline LWA 074 & Inconel 625 & 4392 & 28.259 & 0.0009 & 0.08 & $60 \mathrm{C}$ Aqueous Phase SCW \\
\hline LWA 075 & Inconel 625 & 4392 & 28.267 & 0.0006 & 0.05 & $60 \mathrm{C}$ Aqueous Phase SCw \\
\hline LWA 076 & Inconel 625 & 4392 & 28.453 & 0.0012 & 0.10 & $60 \mathrm{C}$ Aqueous Phase SCW \\
\hline LWA 101 & Inconel 625 & 4392 & 28.252 & 0.0013 & 0.11 & 60 C Water Line SCW \\
\hline LWJ 071 & Inconel 625 & 4392 & 27.890 & 0.0004 & 0.03 & 60 C Vapor Phase SCW \\
\hline LWJ 072 & Inconel 625 & 4392 & 27.498 & 0.0009 & 0.08 & $60 \mathrm{C}$ Vapor Phase SCW \\
\hline LWJ 073 & Inconel 625 & 4392 & 27.672 & 0.0006 & 0.05 & 60 C Vapor Phase SCW \\
\hline LWJ 074 & Inconel 625 & 4392 & 27.847 & 0.0012 & 0.10 & $60 \mathrm{C}$ Aqueous Phase SCW \\
\hline
\end{tabular}




\section{Corrosion Rate Calculations for Weight Loss Specimens}

For Rack Number : 27-1

\begin{tabular}{|c|c|c|c|c|c|c|}
\hline $\begin{array}{l}\text { Sample } \\
\text { Identification }\end{array}$ & Allov & $\begin{array}{l}\text { Lag Time } \\
\text { (hours) }\end{array}$ & $\begin{array}{r}\text { Surface Area } \\
(s q \mathrm{~cm})\end{array}$ & $\begin{array}{l}\text { Weight } \\
\text { Loss }(g)\end{array}$ & $\begin{array}{l}\text { Corrosion Rate } \\
\text { (microns/year) }\end{array}$ & Test Environment \\
\hline LWJ 075 & Inconel 625 & 4392 & 27.503 & 0.0010 & 0.09 & $60 \mathrm{C}$ Aqueous Phase SCW \\
\hline LWJ 076 & Inconel 625 & 4392 & 27.640 & 0.0007 & 0.06 & $60 \mathrm{C}$ Aqueous Phase SCW \\
\hline LWJ 101 & Inconel 625 & 4392 & 27.751 & 0.0006 & 0.05 & $60 \mathrm{C}$ Water Line SCW \\
\hline
\end{tabular}




\section{Corrosion Rate Calculations for Weight Loss Specimens}

For Rack Number : 28-1

$\begin{array}{llll}\text { Sample Allov } & \text { Lag Time Surface Area Weight Corrosion Rate Test Environment } \\ \text { Identification } & \text { (hours) } & \text { (sq cm) Loss }(\mathrm{g}) \text { (microns/year) }\end{array}$

\begin{tabular}{|c|c|c|c|c|c|c|}
\hline AWA 106 & 1825 & 4464 & 28196 & 0.0007 & 0.06 & $90 \mathrm{C}$ Water Line SCW \\
\hline AWA 111 & 1825 & 4464 & 28.252 & 0.0002 & 0.02 & 90 C Vapor Phase SCW \\
\hline AWA 112 & 1825 & 4464 & 28.122 & 0.0005 & 0.04 & $90 \mathrm{C}$ Vapor Phase SCW \\
\hline AWA 113 & 1825 & 4464 & 27.889 & 0.0003 & 0.03 & $90 \mathrm{C}$ Vapor Phase SCW \\
\hline AWA 114 & 1825 & 4464 & 28.106 & 0.0002 & 0.02 & $90 \mathrm{C}$ Aqueous Phase SCW \\
\hline AWA 115 & 1825 & 4464 & 28.425 & 0.0000 & 0.00 & $90 \mathrm{C}$ Aqueous Phase SCW \\
\hline AWA 116 & 1825 & 4464 & 28.130 & 0.0004 & 0.03 & $90 \mathrm{C}$ Aqueous Phase SCW \\
\hline AWB 106 & 1825 & 4464 & 27.442 & 0.0001 & 0.01 & $90 \mathrm{C}$ Water Line SCW \\
\hline AWB 111 & 1825 & 4464 & 27.286 & 0.0003 & 0.03 & $90 \mathrm{C}$ Vapor Phase SCW \\
\hline AWB 112 & 1825 & 4464 & 27.355 & 0.0003 & 0.03 & $90 \mathrm{C}$ Vapor Phase SCW \\
\hline AWB 113 & 1825 & 4464 & 27.589 & 0.0004 & 0.03 & $90 \mathrm{C}$ Vapor Phase SCW \\
\hline AWB 114 & 1825 & 4464 & 27.482 & 0.0005 & 0.04 & $90 \mathrm{C}$ Aqueous Phase SCW \\
\hline AWB 115 & 1825 & 4464 & 27.488 & 0.0007 & 0.06 & $90 \mathrm{C}$ Aqueous Phase SCW \\
\hline AWB 116 & 1825 & 4464 & 27.605 & 0.0002 & 0.02 & $90 \mathrm{C}$ Aqueous Phase SCW \\
\hline BWA 106 & G3 & 4464 & 28.530 & -0.0008 & -0.07 & $90 \mathrm{C}$ Water Line SCW \\
\hline BWA 111 & G3 & 4464 & 28.196 & -0.0003 & -0.03 & $90 \mathrm{C}$ Vapor Phase SCW \\
\hline BWA 112 & G3 & 4464 & 28.740 & -0.0003 & -0.02 & $90 \mathrm{C}$ Vapor Phase SCW \\
\hline BWA 113 & G3 & 4464 & 28.537 & 0.0000 & 0.00 & 90 C Vapor Phase SCW \\
\hline BWA 114 & G3 & 4464 & 28.604 & -0.0001 & -0.01 & $90 \mathrm{C}$ Aqueous Phase SCW \\
\hline
\end{tabular}




\section{Corrosion Rate Calculations for Weight Loss Specimens}

For Rack Number : 28-1

Sample Allov Lag Time Surface Area Weight Corrosion Rate Test Environment Identification (hours) (sq cm) Loss (g) (microns/year)

\begin{tabular}{|c|c|c|c|c|c|c|}
\hline BWA 115 & G3 & 4464 & 28577 & -0.0003 & -0.02 & $90 \mathrm{C}$ Aqueous Phase SCW \\
\hline BWA 116 & G3 & 4464 & 28.617 & 0.0003 & 0.02 & $90 \mathrm{C}$ Aqueous Phase SCW \\
\hline BWC 106 & G3 & 4464 & 28.294 & 0.0001 & 0.01 & $90 \mathrm{C}$ Water Line SCW \\
\hline BWC 111 & G3 & 4464 & 28.279 & 0.0003 & 0.03 & $90 \mathrm{C}$ Vapor Phase SCW \\
\hline BWC 112 & G3 & 4464 & 28.177 & 0.0002 & 0.02 & $90 \mathrm{C}$ Vapor Phase SCW \\
\hline BWC 113 & G3 & 4464 & 28.379 & 0.0004 & 0.03 & 90 C Vapor Phase SCW \\
\hline BWC 114 & G3 & 4464 & 28.224 & 0.0004 & 0.03 & $90 \mathrm{C}$ Aqueous Phase SCW \\
\hline BWC 115 & G3 & 4464 & 28.022 & 0.0004 & 0.03 & $90 \mathrm{C}$ Aqueous Phase SCW \\
\hline BWC 116 & G3 & 4464 & 28.369 & 0.0003 & 0.03 & $90 \mathrm{C}$ Aqueous Phase SCW \\
\hline CWA 106 & $\mathrm{C} 4$ & 4464 & 27.895 & 0.0001 & 0.01 & $90 \mathrm{C}$ Water Line SCW \\
\hline CWA 111 & $\mathrm{C} 4$ & 4464 & 28.306 & 0.0001 & 0.01 & $90 \mathrm{C}$ Vapor Phase SCW \\
\hline CWA 112 & $\mathrm{C} 4$ & 4464 & 28.200 & 0.0003 & 0.02 & 90 C Vapor Phase SCW \\
\hline CWA 113 & $\mathrm{C}_{4}$ & 4464 & 28.055 & -0.0001 & -0.01 & $90 \mathrm{C}$ Vapor Phase SCW \\
\hline CWA 114 & $\mathrm{C} 4$ & 4464 & 27.963 & 0.0008 & 0.07 & $90 \mathrm{C}$ Aqueous Phase SCW \\
\hline CWA 115 & C4 & 4464 & 27.905 & 0.0003 & 0.02 & $90 \mathrm{C}$ Aqueous Phase SCW \\
\hline CWA 116 & $\mathrm{C4}$ & 4464 & 28.166 & 0.0011 & 0.09 & $90 \mathrm{C}$ Aqueous Phase SCW \\
\hline CWB 106 & $\mathrm{C4}$ & 4464 & 27.556 & 0.0008 & 0.07 & $90 \mathrm{C}$ Water Line SCW \\
\hline CWB 111 & $\mathrm{C}_{4}$ & 4464 & 27.652 & 0.0001 & 0.01 & 90 C Vapor Phase SCW \\
\hline CWB 112 & $\mathrm{C} 4$ & 4464 & 27.727 & 0.0000 & 0.00 & 90 C Vapor Phase SCW \\
\hline
\end{tabular}




\section{Corrosion Rate Calculations for Weight Loss Specimens}

For Rack Number : 28-1

$\begin{array}{lcrl}\text { Sample Allov } & \text { Lag Time Surface Area Weight Corrosion Rate Test Environment } \\ \text { Identification } & \text { (hours) } & \text { (sq cm) Loss (g) (microns/year) }\end{array}$

\begin{tabular}{|c|c|c|c|c|c|c|}
\hline CWB 113 & $\mathrm{C} 4$ & 4464 & 27.684 & -0.0001 & -0.01 & $90 \mathrm{C}$ Vapor Phase SCW \\
\hline CWB 114 & $\mathrm{C} 4$ & 4464 & 28.026 & 0.0005 & 0.04 & $90 \mathrm{C}$ Aqueous Phase SCW \\
\hline CWB 115 & $\mathrm{C} 4$ & 4464 & 27.863 & 0.0004 & 0.03 & $90 \mathrm{C}$ Aqueous Phase SCW \\
\hline CWB 116 & $\mathrm{C}_{4}$ & 4464 & 27.769 & 0.0004 & 0.03 & $90 \mathrm{C}$ Aqueous Phase SCW \\
\hline DWA 106 & $\mathrm{C} 22$ & 4464 & 28.121 & 0.0004 & 0.03 & $90 \mathrm{C}$ Water Line SCW \\
\hline DWA 111 & $\mathrm{C} 22$ & 4464 & 28.134 & 0.0003 & 0.02 & $90 \mathrm{C}$ Vapor Phase SCW \\
\hline DWA 112 & $\mathrm{C} 22$ & 4464 & 28.363 & 0.0002 & 0.02 & $90 \mathrm{C}$ Vapor Phase SCW \\
\hline DWA 113 & $\mathrm{C} 22$ & 4464 & 28.235 & 0.0002 & 0.02 & $90 \mathrm{C}$ Vapor Phase SCW \\
\hline DWA 114 & $\mathrm{C} 22$ & 4464 & 28.238 & -0.0002 & -0.02 & $90 \mathrm{C}$ Aqueous Phase SCW \\
\hline DWA 115 & $\mathrm{C} 22$ & 4464 & 28.115 & 0.0002 & 0.02 & $90 \mathrm{C}$ Aqueous Phase SCW \\
\hline DWA 116 & $\mathrm{C} 22$ & 4464 & 28.066 & 0.0004 & 0.03 & $90 \mathrm{C}$ Aqueous Phase SCW \\
\hline DWB 106 & C22 & 4464 & 27.643 & 0.0004 & 0.03 & $90 \mathrm{C}$ Water Line SCW \\
\hline DWB 111 & $\mathrm{C} 22$ & 4464 & 27.449 & 0.0005 & 0.04 & 90 C Vapor Phase SCW \\
\hline DWB 112 & $\mathrm{C} 22$ & 4464 & 27.333 & 0.0007 & 0.06 & 90 C Vapor Phase SCW \\
\hline DWB 113 & $\mathrm{C} 22$ & 4464 & 27.469 & 0.0004 & 0.03 & 90 C Vapor Phase SCW \\
\hline DWB 114 & C22 & 4464 & 27.059 & 0.0009 & 0.08 & $90 \mathrm{C}$ Aqueous Phase SCW \\
\hline DWB 115 & C22 & 4464 & 27.041 & 0.0007 & 0.06 & $90 \mathrm{C}$ Aqueous Phase SCW \\
\hline DWB 116 & $\mathrm{C22}$ & 4464 & 27.440 & 0.0008 & 0.07 & $90 \mathrm{C}$ Aqueous Phase SCW \\
\hline 106 & Ti Gra & 4464 & 28.099 & 0.0376 & 5.93 & $90 \mathrm{C}$ Water Line SCW \\
\hline
\end{tabular}




\section{Corrosion Rate Calculations for Weight Loss Specimens}

For Rack Number : 28-1

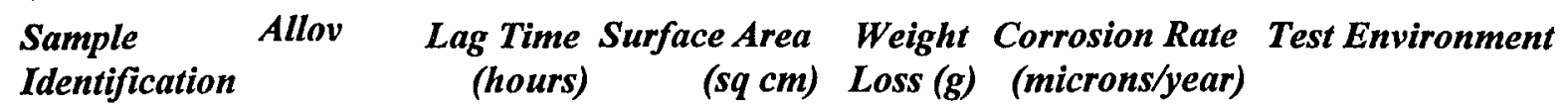

\begin{tabular}{|c|c|c|c|c|c|c|}
\hline EWA 111 & Ti Grade 12 & 4464 & 28.342 & 0.0207 & 3.24 & 90 C Vapor Phase SCW \\
\hline EWA 112 & Ti Grade 12 & 4464 & 28.584 & 0.0261 & 404 & $90 \mathrm{C}$ Vapor Phase SCW \\
\hline EWA 113 & Ti Grade 12 & 4464 & 28.600 & 00309 & 4.79 & $90 \mathrm{C}$ Vapor Phase SCW \\
\hline EWA 114 & Ti Grade 12 & 4464 & 27.978 & 00397 & 6.29 & $90 \mathrm{C}$ Aqueous Phase SCW \\
\hline EWA 115 & Ti Grade 12 & 4464 & 28.646 & 0.0495 & 7.65 & $90 \mathrm{C}$ Aqueous Phase SCW \\
\hline EWA 116 & Ti Grade 12 & 4464 & 28.649 & 0.0406 & 6.28 & $90 \mathrm{C}$ Aqueous Phase SCW \\
\hline EWD 106 & Ti Grade 12 & 4464 & 29.083 & 0.0815 & 12.41 & $90 \mathrm{C}$ Water Line SCW \\
\hline EWD 111 & Ti Grade 12 & 4464 & 28.819 & 0.0316 & 4.86 & $90 \mathrm{C}$ Vapor Phase SCW \\
\hline EWD 112 & Ti Grade 12 & 4464 & 29.210 & 0.0411 & 6.23 & $90 \mathrm{C}$ Vapor Phase SCW \\
\hline EWD 113 & Ti Grade 12 & 4464 & 28.963 & 0.0244 & 3.73 & 90 C Vapor Phase SCW \\
\hline EWD 114 & Ti Grade 12 & 4464 & 28.952 & 0.0426 & 6.52 & $90 \mathrm{C}$ Aqueous Phase SCW \\
\hline EWD 115 & Ti Grade 12 & 4464 & 28.705 & 0.2063 & 31.84 & $90 \mathrm{C}$ Aqueous Phase SCW \\
\hline EWD 116 & Ti Grade 12 & 4464 & 29.114 & 0.0631 & 9.60 & $90 \mathrm{C}$ Aqueous Phase SCW \\
\hline FWA 106 & Ti Grade 16 & 4464 & 27.798 & 0.0241 & 3.76 & $90 \mathrm{C}$ Water Line SCW \\
\hline FWA 111 & Ti Grade 16 & 4464 & 27.795 & 0.0290 & 4.53 & $90 \mathrm{C}$ Vapor Phase SCW \\
\hline FWA 112 & Ti Grade 16 & 4464 & 27.633 & 0.0640 & 10.06 & 90 C Vapor Phase SCW \\
\hline FWA 113 & Ti Grade 16 & 4464 & 27.586 & 0.0295 & 4.64 & 90 C Vapor Phase SCW \\
\hline FWA 114 & Ti Grade 16 & 4464 & 27.458 & 0.0361 & 5.71 & $90 \mathrm{C}$ Aqueous Phase SCW \\
\hline FWA 115 & Ti Grade 16 & 4464 & 27.529 & 0.0314 & 4.95 & $90 \mathrm{C}$ Aqueous Phase SCW \\
\hline
\end{tabular}




\section{Corrosion Rate Calculations for Weight Loss Specimens}

For Rack Number : 28-1

$\begin{array}{llll}\text { Sample Allov } & \text { Lag Time Surface Area Weight Corrosion Rate Test Environment } \\ \text { Identification } & \text { (hours) } & (s q \mathrm{~cm}) & \text { Loss }(\mathrm{g}) \text { (microns/year) }\end{array}$

\begin{tabular}{|c|c|c|c|c|c|c|}
\hline FWA 116 & Ti Grade 16 & 4464 & 27.639 & 0.0343 & 5.39 & $90 \mathrm{C}$ Aqueous Phase SCW \\
\hline FWE 106 & Ti Grade 16 & 4464 & 27.486 & 00294 & 4.64 & $90 \mathrm{C}$ Water Line SCW \\
\hline FWE 111 & Ti Grade 16 & 4464 & 28.211 & 0.0292 & 4.49 & $90 \mathrm{C}$ Vapor Phase SCW \\
\hline FWE 112 & TiGrade 16 & 4464 & 27.848 & 0.0192 & 2.99 & $90 \mathrm{C}$ Vapor Phase SCW \\
\hline FWE 113 & Ti Grade 16 & 4464 & 28.109 & 0.0289 & 4.46 & 90 C Vapor Phase SCW \\
\hline FWE 114 & Ti Grade 16 & 4464 & 28.025 & 0.0326 & 5.05 & $90 \mathrm{C}$ Aqueous Phase SCW \\
\hline FWE 115 & Ti Grade 16 & 4464 & 27.741 & 0.0831 & 13.01 & $90 \mathrm{C}$ Aqueous Phase SCW \\
\hline FWE 116 & Ti Grade 16 & 4464 & 27.997 & 0.0362 & 5.61 & $90 \mathrm{C}$ Aqueous Phase SCW \\
\hline LWA 106 & inconel 625 & 4464 & 28.327 & 00005 & 0.04 & $90 \mathrm{C}$ Water Line ScW \\
\hline LWA 111 & Inconel 625 & 4464 & 28.095 & 0.0004 & 0.03 & $90 \mathrm{C}$ Vapor Phase SCW \\
\hline LWA 112 & Inconel 625 & 4464 & 28.266 & 0.0004 & 0.03 & $90 \mathrm{C}$ Vapor Phase SCW \\
\hline LWA 113 & Inconel 625 & 4464 & 28.247 & 0.0004 & 0.03 & $90 \mathrm{C}$ Vapor Phase SCW \\
\hline LWA 114 & Inconel 625 & 4464 & 28.168 & 0.0006 & 0.05 & $90 \mathrm{C}$ Aqueous Phase SCW \\
\hline LWA 115 & Inconel 625 & 4464 & 28.213 & 0.0009 & 0.07 & $90 \mathrm{C}$ Aqueous Phase SCW \\
\hline LWA 116 & Inconel 625 & 4464 & 28.123 & 0.0005 & 0.04 & $90 \mathrm{C}$ Aqueous Phase SCW \\
\hline LWJ 106 & Inconel 625 & 4464 & 27.728 & 0.0003 & 0.03 & $90 \mathrm{C}$ Water Line SCW \\
\hline LWJ 111 & Inconel 625 & 4464 & 27.653 & 0.0003 & 0.03 & $90 \mathrm{C}$ Vapor Phase SCW \\
\hline LWJ 112 & Inconel 625 & 4464 & 27.557 & -0.0002 & -0.02 & 90 C Vapor Phase SCW \\
\hline LWJ 113 & Inconel 625 & 4464 & 27.776 & 0.0002 & 0.02 & 90 C Vapor Phase SCW \\
\hline
\end{tabular}




\section{Corrosion Rate Calculations for Weight Loss Specimens}

For Rack Number : 28-1

Sample Allov Lag Time Surface Area Weight Corrosion Rate Test Environment

Identification (hours) (sq cm) Loss (g) (microns/year)

\begin{tabular}{lrrrrrr}
\hline LWJ 114 & Inconel 625 & 4464 & 27.633 & 0.0005 & 0.04 & $90 \mathrm{C}$ Aqueous Phase SCW \\
LWJ 115 & Inconel 625 & 4464 & 27.592 & 0.0005 & 0.04 & $90 \mathrm{C}$ Aqueous Phase SCW \\
LWJ 116 & Inconel 625 & 4464 & 27.807 & 0.0003 & 0.03 & $90 \mathrm{C}$ Aqueous Phase SCW
\end{tabular}




\section{Corrosion Rate Calculations for Weight Loss Specimens}

For Rack Number : 29-1

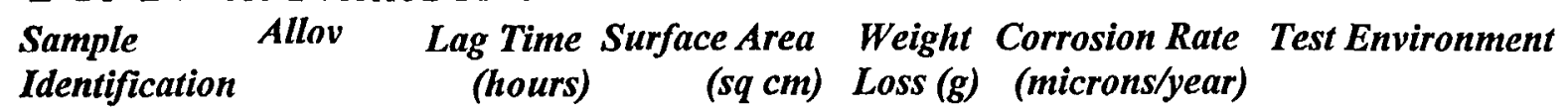

\begin{tabular}{|c|c|c|c|c|c|c|}
\hline AWA 141 & 1825 & 4464 & 28.374 & -0.0002 & -0.02 & 60 C Vapor Phase SDW \\
\hline AWA 142 & 1825 & 4464 & 28.016 & -0.0001 & -0.01 & $60 \mathrm{C}$ Aqueous Phase SDW \\
\hline AWA 151 & 1825 & 4464 & 28.406 & -0.0003 & -0.03 & $60 \mathrm{C}$ Water Line SDW \\
\hline AWB 141 & 1825 & 4464 & 27.028 & 0.0003 & 0.03 & $60 \mathrm{C}$ Vapor Phase SDW \\
\hline AWB 142 & 1825 & 4464 & 27.380 & 0.0005 & 0.04 & $60 \mathrm{C}$ Aqueous Phase SDW \\
\hline AWB 151 & 1825 & 4464 & 27.699 & 0.0004 & 0.03 & $60 \mathrm{C}$ Water Line SDW \\
\hline BWA 141 & G3 & 4464 & 28.571 & 0.0006 & 0.05 & $60 \mathrm{C}$ Vapor Phase SDW \\
\hline BWA 142 & G3 & 4464 & 28.679 & 00005 & 0.04 & $60 \mathrm{C}$ Aqueous Phase SDW \\
\hline BWA 151 & G3 & 4464 & 28.378 & -0.0002 & -0.02 & $60 \mathrm{C}$ Water Line SDW \\
\hline BWC 141 & G3 & 4464 & 28.239 & 0.0001 & 0.01 & $60 \mathrm{C}$ Vapor Phase SDW \\
\hline BWC 142 & G3 & 4464 & 28.349 & 0.0002 & 0.02 & $60 \mathrm{C}$ Aqueous Phase SDW \\
\hline BWC 151 & G3 & 4464 & 28.278 & -0.0001 & -0.01 & $60 \mathrm{C}$ Water Line SDW \\
\hline CWA 141 & C4 & 4464 & 28.362 & 0.0006 & 0.05 & $60 \mathrm{C}$ Vapor Phase SDW \\
\hline CWA 142 & C4 & 4464 & 28.095 & 0.0002 & 0.02 & $60 \mathrm{C}$ Aqueous Phase SDW \\
\hline CWA 151 & C4 & 4464 & 27.919 & 0.0000 & 0.00 & $60 \mathrm{C}$ Water Line SDW \\
\hline CWB 141 & $\mathrm{C} 4$ & 4464 & 27.871 & 0.0004 & 0.03 & 60 C Vapor Phase SDW \\
\hline CWB 142 & C4 & 4464 & 27.989 & 0.0003 & 0.02 & $60 \mathrm{C}$ Aqueous Phase SDW \\
\hline CWB 151 & C4 & 4464 & 28.193 & 0.0000 & 0.00 & $60 \mathrm{C}$ Water Line SDW \\
\hline DWA 141 & C22 & 4464 & 28.205 & -0.0001 & -0.01 & $60 \mathrm{C}$ Vapor Phase SDW \\
\hline
\end{tabular}




\section{Corrosion Rate Calculations for Weight Loss Specimens}

For Rack Number : 29-1

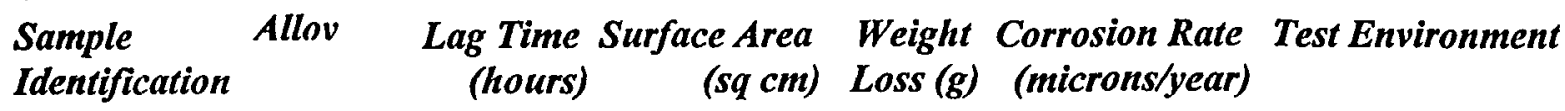

\begin{tabular}{|c|c|c|c|c|c|c|}
\hline DWA 142 & $\mathrm{C} 22$ & 4464 & 28.208 & -0.0002 & -0.02 & $60 \mathrm{C}$ Aqueous Phase SDW \\
\hline DWA 151 & $\mathrm{C} 22$ & 4464 & 28135 & 00001 & 0.01 & $60 \mathrm{C}$ Water Line SDW \\
\hline DWB 141 & $\mathrm{C} 22$ & 4464 & 27.732 & 0.0003 & 0.02 & $60 \mathrm{C}$ Vapor Phase SDW \\
\hline DWB 142 & $\mathrm{C} 22$ & 4464 & 27.441 & 0.0001 & 0.01 & $60 \mathrm{C}$ Aqueous Phase SDW \\
\hline DWB 151 & $\mathrm{C} 22$ & 4464 & 27.674 & 0.0001 & 0.01 & $60 \mathrm{C}$ Water Line SDW \\
\hline EWA 141 & Ti Grade 12 & 4464 & 28.371 & 0.0296 & 4.62 & $60 \mathrm{C}$ Vapor Phase SDW \\
\hline EWA 142 & Ti Grade 12 & 4464 & 29.038 & 0.0236 & 3.60 & $60 \mathrm{C}$ Aqueous Phase SDW \\
\hline EWA 151 & Ti Grade 12 & 4464 & 29.116 & 0.0327 & 4.98 & $60 \mathrm{C}$ Water Line SDW \\
\hline EWD 141 & Ti Grade 12 & 4464 & 29044 & 0.0260 & 3.97 & $60 \mathrm{C}$ Vapor Phase SDW \\
\hline EWD 142 & Ti Grade 12 & 4464 & 28.926 & 0.0617 & 9.45 & $60 \mathrm{C}$ Aqueous Phase SOW \\
\hline EWD 151 & Ti Grade 12 & 4464 & 28.988 & 0.0399 & 6.10 & $60 \mathrm{C}$ Water Line SDW \\
\hline FWA 141 & Ti Grade 16 & 4464 & 27.806 & 0.0249 & 3.89 & 60 C Vapor Phase SDW \\
\hline FWA 142 & Ti Grade 16 & 4464 & 27.626 & 0.0238 & 3.74 & $60 \mathrm{C}$ Aqueous Phase SDW \\
\hline FWA 151 & Ti Grade 16 & 4464 & 27.717 & 0.0608 & 9.52 & $60 \mathrm{C}$ Water Line SDW \\
\hline FWE 141 & Ti Grade 16 & 4464 & 27.668 & 0.0158 & 2.48 & 60 C Vapor Phase SDW \\
\hline FWE 142 & Ti Grade 16 & 4464 & 27.571 & 0.0267 & 4.20 & $60 \mathrm{C}$ Aqueous Phase SDW \\
\hline FWE 151 & Ti Grade 16 & 4464 & 27.871 & 0.0271 & 4.22 & $60 \mathrm{C}$ Water Line SDW \\
\hline LWA 141 & Inconel 625 & 4464 & 28.311 & 0.0009 & 0.07 & $60 \mathrm{C}$ Vapor Phase SDW \\
\hline LWA 142 & Inconel 625 & 4464 & 28.336 & 0.0006 & 0.05 & $60 \mathrm{C}$ Aqueous Phase SDW \\
\hline
\end{tabular}




\section{Corrosion Rate Calculations for Weight Loss Specimens}

For Rack Number : 29-1

\begin{tabular}{llccccc}
$\begin{array}{l}\text { Sample } \\
\text { Identification }\end{array}$ & Allov & $\begin{array}{c}\text { Lag Time Surface Area } \\
\text { (hours) }\end{array}$ & $\begin{array}{c}\text { Weight } \\
\text { (sq } \mathrm{cm} \text { ) }\end{array}$ & $\begin{array}{c}\text { Corrosion Rate } \\
\text { Loss }(\mathrm{g})\end{array}$ & Test Envicrons/year) & \\
\hline LWA 151 & Inconel 625 & 4464 & 28.247 & 0.0002 & 0.02 & 60 C Water Line SDW \\
LWJ 141 & Inconel 625 & 4464 & 27.764 & 0.0002 & 0.02 & 60 C Vapor Phase SDW \\
LWJ 142 & Inconel 625 & 4464 & 27.774 & 0.0002 & 0.02 & 60 C Aqueous Phase SDW \\
LWJ 151 & Inconel 625 & 4464 & 27.861 & 0.0000 & 0.00 & 60 C Water Line SDW
\end{tabular}




\section{Corrosion Rate Calculations for Weight Loss Specimens}

For Rack Number : 30-1

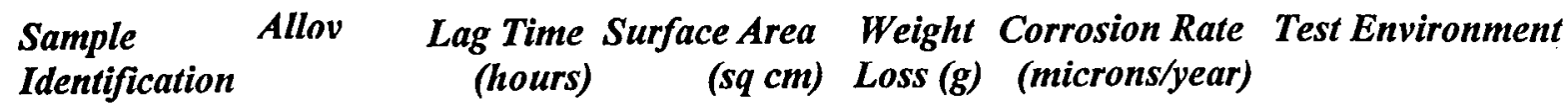

\begin{tabular}{|c|c|c|c|c|c|c|}
\hline AWA 164 & 1825 & 4392 & 28.058 & 0.0002 & 0.02 & $90 \mathrm{C}$ Water Line SDW \\
\hline AWA 168 & 1825 & 4392 & 28.284 & 0.0003 & 0.03 & $90 \mathrm{C}$ Vapor Phase SDW \\
\hline AWA 169 & 1825 & 4392 & 28.222 & 0.0006 & 0.05 & $90 \mathrm{C}$ Aqueous Phase SDW \\
\hline AWB 164 & 1825 & 4392 & 27.750 & 0.0002 & 0.02 & $90 \mathrm{C}$ Water Line SDW \\
\hline AWB 168 & 1825 & 4392 & 27.871 & 0.0004 & 0.04 & 90 C Vapor Phase SDW \\
\hline AWB 169 & 1825 & 4392 & 27.901 & 0.0002 & 0.02 & $90 \mathrm{C}$ Aqueous Phase SDW \\
\hline BWA 164 & G3 & 4392 & 27.817 & 0.0010 & 0.09 & $90 \mathrm{C}$ Water Line SDW \\
\hline BWA 168 & G3 & 4392 & 27.793 & 0.0016 & 0.14 & $90 \mathrm{C}$ Vapor Phase SDW \\
\hline BWA 169 & G3 & 4392 & 27.866 & 0.0014 & 0.12 & $90 \mathrm{C}$ Aqueous Phase SDW \\
\hline BWC 164 & G3 & 4392 & 27.918 & 0.0001 & 0.01 & $90 \mathrm{C}$ Water Line SDW \\
\hline BWC 168 & G3 & 4392 & 27.842 & 0.0000 & 0.00 & $90 \mathrm{C}$ Vapor Phase SDW \\
\hline BWC 169 & G3 & 4392 & 27.900 & 0.0011 & 0.10 & $90 \mathrm{C}$ Aqueous Phase SDW \\
\hline CWA 164 & $\mathrm{C}_{4}$ & 4392 & 28.152 & 0.0011 & 0.09 & $90 \mathrm{C}$ Water Line SDW \\
\hline CWA 168 & $\mathrm{C} 4$ & 4392 & 28.153 & 0.0016 & 0.13 & 90 C Vapor Phase SDW \\
\hline CWA 169 & C4 & 4392 & 28.113 & 0.0012 & 0.10 & $90 \mathrm{C}$ Aqueous Phase SDW \\
\hline CWB 164 & C4 & 4392 & 28.026 & 0.0008 & 0.07 & $80 \mathrm{C}$ Water Line SDW \\
\hline CWB 168 & C4 4 & 4392 & 27.792 & 0.0004 & 0.03 & $90 \mathrm{C}$ Vapor Phase SDW \\
\hline CWB 169 & C4 & 4392 & 27.610 & 0.0011 & 0.09 & $90 \mathrm{C}$ Aqueous Phase SDW \\
\hline DWA 164 & C22 & 4392 & 28.192 & 0.0010 & 0.08 & 90 C Water Line SDW \\
\hline
\end{tabular}




\section{Corrosion Rate Calculations for Weight Loss Specimens}

For Rack Number : 30-1

\section{Sample Allov Lag Time Surface Area Weight Corrosion Rate Test Environment Identification (hours) (sq cm) Loss (g) (microns/year)}

\begin{tabular}{|c|c|c|c|c|c|c|}
\hline DWA 168 & $\mathrm{C} 22$ & 4392 & 28.067 & 0.0004 & 0.03 & $90 \mathrm{C}$ Vapor Phase SDW \\
\hline DWA 169 & $\mathrm{C} 22$ & 4392 & 27.989 & 0.0009 & 0.07 & $90 \mathrm{C}$ Aqueous Phase SDW \\
\hline DWB 164 & $\mathrm{C} 22$ & 4392 & 27.609 & 0.0002 & 0.02 & $90 \mathrm{C}$ Water Line SDW \\
\hline DWB 168 & $\mathrm{C} 22$ & 4392 & 27.763 & 0.0008 & 0.07 & $90 \mathrm{C}$ Vapor Phase SDW \\
\hline DWB 169 & $\mathrm{C} 22$ & 4392 & 27.238 & 0.0003 & 0.03 & $90 \mathrm{C}$ Aqueous Phase SDW \\
\hline EWA 164 & Ti Grade 12 & 4392 & 27.803 & 00349 & 5.65 & $90 \mathrm{C}$ Water Line SDW \\
\hline EWA 168 & Ti Grade 12 & 4392 & 27.722 & 0.0377 & 6.12 & $90 \mathrm{C}$ Vapor Phase SDW \\
\hline EWA 169 & Ti Grade 12 & 4392 & 27.650 & 0.0328 & 5.34 & $90 \mathrm{C}$ Aqueous Phase SDW \\
\hline EWD 164 & Ti Grade 12 & 4392 & 27.371 & 0.0331 & 5.44 & $90 \mathrm{C}$ Water Line SDW \\
\hline EWD 168 & Ti Grade 12 & 4392 & 27.655 & 0.0338 & 5.50 & $90 \mathrm{C}$ Vapor Phase SDW \\
\hline EWD 169 & TiGrade 12 & 4392 & 37.862 & 0.0386 & 4.59 & $90 \mathrm{C}$ Aqueous Phase SDW \\
\hline FWA 164 & Ti Grade 16 & 4392 & 27.441 & 0.0262 & 4.21 & $90 \mathrm{C}$ Water Line SDW \\
\hline FWA 168 & Ti Grade 16 & 4392 & 27.239 & 0.0245 & 3.97 & 90 C Vapor Phase SDW \\
\hline FWA 169 & Ti Grade 16 & 4392 & 27.444 & 0.0236 & 3.79 & $90 \mathrm{C}$ Aqueous Phase SDW \\
\hline FWE 164 & Ti Grade 16 & 4392 & 27.380 & 0.0253 & 4.08 & $90 \mathrm{C}$ Water Line SDW \\
\hline FWE 168 & Ti Grade 16 & 4392 & 27.597 & 0.0177 & 2.83 & $90 \mathrm{C}$ Vapor Phase SDW \\
\hline FWE 169 & Ti Grade 16 & 4392 & 27.572 & 0.0472 & 7.55 & $90 \mathrm{C}$ Aqueous Phase SDW \\
\hline LWA 164 & Inconel 625 & 4392 & 28.253 & 0.0007 & 0.06 & $90 \mathrm{C}$ Water Line SDW \\
\hline LWA 168 & Inconel 625 & 4392 & 28.195 & 0.0005 & 0.04 & $90 \mathrm{C}$ Vapor Phase SDW \\
\hline
\end{tabular}




\section{Corrosion Rate Calculations for Weight Loss Specimens}

For Rack Number : 30-1

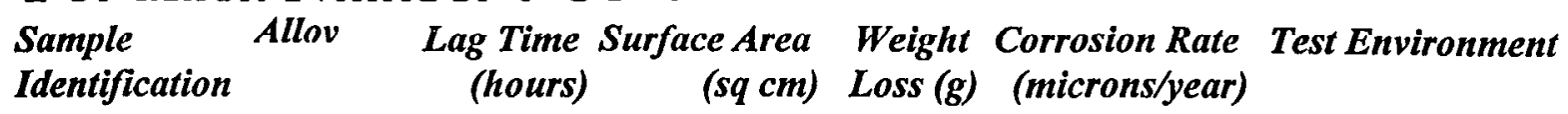

\begin{tabular}{lrrrrrr}
\hline LWA 169 & Inconel 625 & 4392 & 28.106 & 00012 & 0.10 & 90 C Aqueous Phase SDW \\
LWJ 164 & Inconel 625 & 4392 & 27773 & 0.0002 & 0.02 & 90 C Water Line SDW \\
LWJ 168 & Inconel 625 & 4392 & 27.750 & 0.0008 & 0.07 & 90 C Vapor Phase SDW \\
LWJ 169 & Inconel 625 & 4392 & 27.749 & 0.0004 & 0.03 & 90 C Aqueous Phase SDW
\end{tabular}




\section{Corrosion Rate Calculations for Crevice Specimens For Rack Number : 23-1 \\ Sample Allov Lag Time Surface Area Weight Corrosion Rate Test Environment Identification (hours) (sq cm) Loss (g) (microns/year)}

\begin{tabular}{|c|c|c|c|c|c|c|}
\hline ICA 001 & $\begin{array}{l}\text { A387 } \\
\text { Grade } 22\end{array}$ & 4536 & 53.025 & 0.2703 & 12.52 & $60 \mathrm{C}$ SDW VAPOR PHASE \\
\hline ICA 002 & $\begin{array}{l}\text { A387 } \\
\text { Grade } 22\end{array}$ & 4536 & 53.077 & 0.2546 & 11.79 & $60 \mathrm{C}$ SDW VAPOR PHASE \\
\hline ICA 003 & $\begin{array}{l}\text { A387 } \\
\text { Grade } 22\end{array}$ & 4536 & 53.257 & 0.2455 & 11.33 & GOC SDW VAPOR PHASE \\
\hline ICA 004 & $\begin{array}{l}\text { A387 } \\
\text { Grade } 22\end{array}$ & 4536 & 52.952 & 2.1500 & 99.76 & $60 \mathrm{C}$ SDW AQUEOUS PHASE \\
\hline ICA 005 & $\begin{array}{l}\text { A387 } \\
\text { Grade } 22\end{array}$ & 4536 & 53.027 & 2.2049 & 102.16 & $60 \mathrm{C}$ SDW AQUEOUS PHASE \\
\hline ICA 006 & $\begin{array}{l}\text { A387 } \\
\text { Grade } 22\end{array}$ & 4536 & 52.736 & 2.1720 & 101.20 & $60 \mathrm{C}$ SDW AQUEOUS PHASE \\
\hline ICH 001 & $\begin{array}{l}\text { A387 } \\
\text { Grade } 22\end{array}$ & 4536 & 53.003 & 0.3047 & 14.12 & $60 \mathrm{C}$ SDW VAPOR PHASE \\
\hline $\mathrm{ICHOO2}$ & $\begin{array}{l}\text { A387 } \\
\text { Grade } 22\end{array}$ & 4536 & 53.055 & 0.3523 & 16.32 & $60 \mathrm{C}$ SDW VAPOR PHASE \\
\hline $\mathrm{ICH} 003$ & $\begin{array}{l}\text { A387 } \\
\text { Grade } 22\end{array}$ & 4536 & 52.881 & 0.6660 & 30.94 & $60 \mathrm{C}$ SDW VAPOR PHASE \\
\hline ICH 004 & $\begin{array}{l}\text { A387 } \\
\text { Grade } 22\end{array}$ & 4536 & 52.986 & 2.4095 & 111.73 & $60 \mathrm{C}$ SDW AQUEOUS PHASE \\
\hline ICH 005 & $\begin{array}{l}\text { A387 } \\
\text { Grade } 22\end{array}$ & 4536 & 53.000 & 1.7030 & 78.95 & $60 \mathrm{C}$ SDW AQUEOUS PHASE \\
\hline ICH 006 & $\begin{array}{l}\text { A387 } \\
\text { Grade } 22\end{array}$ & 4536 & 53.069 & 1.8889 & 87.45 & $60 \mathrm{C}$ SDW AQUEOUS PHASE \\
\hline JCA 001 & $\begin{array}{l}\text { A516 } \\
\text { Grade } 55\end{array}$ & 4536 & 52.871 & 1.3607 & 63.23 & $60 \mathrm{C}$ SDW VAPOR PHASE \\
\hline $\mathrm{SCA} 002$ & $\begin{array}{l}\text { A516 } \\
\text { Grade } 55\end{array}$ & 4536 & 52.573 & 1.1356 & 53.07 & $60 \mathrm{C}$ SDW VAPOR PHASE \\
\hline$J C A 003$ & $\begin{array}{l}\text { A516 } \\
\text { Grade } 55\end{array}$ & 4536 & 53.493 & 1.0760 & 49.42 & $60 \mathrm{C}$ SDW VAPOR PHASE \\
\hline JCA 004 & $\begin{array}{l}\text { A516 } \\
\text { Grade } 55\end{array}$ & 4536 & 52.719 & 1.8839 & 87.80 & 60 C SDW AQUEOUS PHASE \\
\hline JCA 005 & $\begin{array}{l}\text { A516 } \\
\text { Grade } 55\end{array}$ & 4536 & 52.805 & 1.9011 & 88.29 & $60 \mathrm{C}$ SDW AQUEOUS PHASE \\
\hline JCA 006 & $\begin{array}{l}\text { A516 } \\
\text { Grade } 55\end{array}$ & 4536 & 53.481 & 1.7132 & 78.71 & $60 \mathrm{C}$ SDW AQUEOUS PHASE \\
\hline $\mathrm{scl} 001$ & $\begin{array}{l}\text { A516 } \\
\text { Grade } 55\end{array}$ & 4536 & 51.695 & 1.1230 & 53.38 & $60 \mathrm{C}$ SDW VAPOR PHASE \\
\hline
\end{tabular}




\section{Corrosion Rate Calculations for Crevice Specimens}

For Rack Number : 23-1 $\begin{array}{llll}\text { Sample Allov } & \text { Lag Time Surface Area Weight Corrosion Rate Test Environment } \\ \text { Identification } & \text { (hours) } & \text { (sq cm) Loss }(g) \text { (microns/year) }\end{array}$

\begin{tabular}{|c|c|c|c|c|c|c|}
\hline $\mathrm{JCl} 002$ & $\begin{array}{l}\text { A516 } \\
\text { Grade } 55\end{array}$ & 4536 & 53.646 & 1.3672 & 62.62 & $60 \mathrm{C}$ SDW VAPOR PHASE \\
\hline $\mathrm{JCl} 003$ & $\begin{array}{l}\text { A516 } \\
\text { Grade } 55\end{array}$ & 4536 & 51.804 & 1.5169 & 71.95 & $60 \mathrm{C}$ SDW VAPOR PHASE \\
\hline $\mathrm{JCl} 004$ & $\begin{array}{l}\text { A516 } \\
\text { Grade } 55\end{array}$ & 4536 & 51.626 & 2.3630 & 112.46 & $60 \mathrm{C}$ SDW AQUEOUS PHASE \\
\hline $\mathrm{JCl} 005$ & $\begin{array}{l}\text { A516 } \\
\text { Grade } 55\end{array}$ & 4536 & 52.042 & 2.1855 & 103.18 & $60 \mathrm{C}$ SDW AQUEOUS PHASE \\
\hline $\mathrm{JCl} 006$ & $\begin{array}{l}\text { A516 } \\
\text { Grade } 55\end{array}$ & 4536 & 52.452 & 2.1291 & 99.73 & $60 \mathrm{C}$ SDW AQUEOUS PHASE \\
\hline KCA 001 & $\begin{array}{l}\text { A27 Grade } \\
70-40\end{array}$ & 4536 & 51.849 & 1.5080 & 71.83 & $60 \mathrm{C}$ SDW VAPOR PHASE \\
\hline KCA 002 & $\begin{array}{l}\text { A27 Grade } \\
70-40\end{array}$ & 4536 & 51.819 & 1.2345 & 58.83 & $60 \mathrm{C}$ SDW VAPOR PHASE \\
\hline KCA 003 & $\begin{array}{l}\text { A27 Grade } \\
70-40\end{array}$ & 4536 & 51.885 & 1.3747 & 65.43 & $60 \mathrm{C}$ SDW VAPOR PHASE \\
\hline KCA 004 & $\begin{array}{l}\text { A27 Grade } \\
70-40\end{array}$ & 4536 & 51.957 & 21528 & 102.33 & $60 \mathrm{C}$ SDW AQUEOUS PHASE \\
\hline KCA 005 & $\begin{array}{l}\text { A27 Grade } \\
70-40\end{array}$ & 4536 & 51.271 & 2.1123 & 101.74 & $60 \mathrm{C}$ SDW AQUEOUS PHASE \\
\hline KCA 006 & $\begin{array}{l}\text { A27 Grade } \\
70-40\end{array}$ & 4536 & 52.142 & 2.7595 & 130.70 & 60 C SDW AQUEOUS PHASE \\
\hline KCl 001 & $\begin{array}{l}\text { A27 Grade } \\
70-40\end{array}$ & 4536 & 52.198 & 1.2639 & 59.80 & $60 \mathrm{C}$ SDW VAPOR PHASE \\
\hline $\mathrm{KCl} 002$ & $\begin{array}{l}\text { A27 Grade } \\
70-40\end{array}$ & 4536 & 51.915 & 1.3488 & 64.16 & $60 \mathrm{C}$ SDW VAPOR PHASE \\
\hline $\mathrm{KCl} 003$ & $\begin{array}{l}\text { A27 Grade } \\
70-40\end{array}$ & 4536 & 52.097 & 1.5664 & 74.25 & $60 \mathrm{C}$ SDW VAPOR PHASE \\
\hline $\mathrm{KCl} 004$ & $\begin{array}{l}\text { A27 Grade } \\
70-40\end{array}$ & 4536 & 52.381 & 2.3001 & 108.44 & $60 \mathrm{C}$ SDW AQUEOUS PHASE \\
\hline $\mathrm{KCl} 005$ & $\begin{array}{l}\text { A27 Grade } \\
70-40\end{array}$ & 4536 & 52.154 & 1.9619 & 92.90 & $60 \mathrm{C}$ SDW AQUEOUS PHASE \\
\hline $\mathrm{KCl} 006$ & $\begin{array}{l}\text { A27 Grade } \\
70-40\end{array}$ & 4536 & 51.435 & 2.5326 & 121.60 & $60 \mathrm{C}$ SDW AQUEOUS PHASE \\
\hline
\end{tabular}




\section{Corrosion Rate Calculations for Crevice Specimens}

\section{For Rack Number : 23-2}

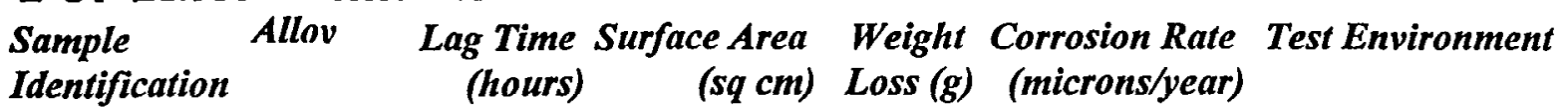

\begin{tabular}{|c|c|c|c|c|c|c|}
\hline ICA 007 & $\begin{array}{l}\text { A387 } \\
\text { Grade } 22\end{array}$ & 8760 & 53.061 & 0.4225 & 10.13 & $60 \mathrm{C}$ SDW VAPOR PHASE \\
\hline ICA 008 & $\begin{array}{l}\text { A387 } \\
\text { Grade } 22\end{array}$ & 8760 & 52.909 & 0.3357 & 8.07 & $60 \mathrm{C}$ SDW VAPOR PHASE \\
\hline ICA 009 & $\begin{array}{l}\text { A387 } \\
\text { Grade 22 }\end{array}$ & 8760 & 52.905 & 0.4473 & 10.76 & $60 \mathrm{C}$ SDW VAPOR PHASE \\
\hline ICA 010 & $\begin{array}{l}\text { A387 } \\
\text { Grade } 22\end{array}$ & 8760 & 52.767 & 3.5142 & 84.73 & $60 \mathrm{C}$ SDW AQUEOUS PHASE \\
\hline ICA 011 & $\begin{array}{l}\text { A387 } \\
\text { Grade } 22\end{array}$ & 8760 & 53.018 & 4.7819 & 114.75 & $60 \mathrm{C}$ SDW AQUEOUS PHASE \\
\hline ICA 012 & $\begin{array}{l}\text { A387 } \\
\text { Grade } 22\end{array}$ & 8760 & 53.128 & 3.9595 & 94.82 & $60 \mathrm{C}$ SDW AQUEOUS PHASE \\
\hline ICH 007 & $\begin{array}{l}\text { A387 } \\
\text { Grade } 22\end{array}$ & 8760 & 53.151 & 0.5510 & 13.19 & $60 \mathrm{C}$ SDW VAPOR PHASE \\
\hline $\mathrm{ICHOOB}$ & $\begin{array}{l}\text { A387 } \\
\text { Grade } 22\end{array}$ & 8760 & 53.173 & 0.6223 & 14.89 & $60 \mathrm{C}$ SDW VAPOR PHASE \\
\hline ICH OO9 & $\begin{array}{l}\text { A387 } \\
\text { Grade } 22\end{array}$ & 8760 & 53.319 & 0.9945 & 23.73 & $60 \mathrm{C}$ SDW VAPOR PHASE \\
\hline ICH 010 & $\begin{array}{l}\text { A387 } \\
\text { Grade } 22\end{array}$ & 8760 & 52.932 & 4.7824 & 114.95 & $60 \mathrm{C}$ SDW AQUEOUS PHASE \\
\hline ICH 011 & $\begin{array}{l}\text { A387 } \\
\text { Grade } 22\end{array}$ & 8760 & 52.515 & 3.6757 & 89.05 & $60 \mathrm{C}$ SDW AQUEOUS PHLSE \\
\hline ICH 012 & $\begin{array}{l}\text { A387 } \\
\text { Grade } 22\end{array}$ & 8760 & 52.713 & 1.4627 & 35.30 & $60 \mathrm{C}$ SDW AQUEOUS PHASE \\
\hline JCA 007 & $\begin{array}{l}\text { A516 } \\
\text { Grade } 55\end{array}$ & 8760 & 53.332 & 1.1052 & 26.37 & $60 \mathrm{C}$ SDW VAPOR PHASE \\
\hline JCA 008 & $\begin{array}{l}\text { A516 } \\
\text { Grade } 55\end{array}$ & 8760 & 53.033 & 0.8082 & 19.39 & $60 \mathrm{C}$ SDW VAPOR PHASE \\
\hline JCA 009 & $\begin{array}{l}\text { A516 } \\
\text { Grade } 55\end{array}$ & 8760 & 52.975 & 0.8234 & 19.78 & $60 \mathrm{C}$ SDW VAPOR PHASE \\
\hline JCA 010 & $\begin{array}{l}\text { A516 } \\
\text { Grade } 55\end{array}$ & 8760 & 52.562 & 2.9437 & 71.25 & $60 \mathrm{C}$ SDW AQUEOUS PHASE \\
\hline JCA 011 & $\begin{array}{l}\text { A516 } \\
\text { Grade } 55\end{array}$ & 8760 & 52.293 & 2.7033 & 65.77 & $60 \mathrm{C}$ SDW AQUEOUS PHASE \\
\hline JCA 012 & $\begin{array}{l}\text { A516 } \\
\text { Grade } 55\end{array}$ & 8760 & 52.677 & 2.8983 & 70.00 & $60 \mathrm{C}$ SDW AQUEOUS PHASE \\
\hline $\mathrm{JCl} 007$ & $\begin{array}{l}\text { A516 } \\
\text { Grade } 55\end{array}$ & 8760 & 51.940 & 1.1230 & 27.51 & $60 \mathrm{C}$ SDW VAPOR PHASE \\
\hline
\end{tabular}




\section{Corrosion Rate Calculations for Crevice Specimens}

For Rack Number : 23-2

Sample Allov Lag Time Surface Area Weight Corrosion Rate Test Environment Identification (hours) (sq cm) Loss (g) (microns/year)

\begin{tabular}{|c|c|c|c|c|c|c|}
\hline $\mathrm{JCl} 008$ & $\begin{array}{l}\text { A516 } \\
\text { Grade } 55\end{array}$ & 8760 & 51.640 & 1.1154 & 27.48 & $60 \mathrm{C}$ SDW VAPOR PHASE \\
\hline $\mathrm{JCl} 009$ & $\begin{array}{l}\text { A516 } \\
\text { Grade } 55\end{array}$ & 8760 & 51.560 & 1.5948 & 39.35 & $60 \mathrm{C}$ SDW VAPOR PHASE \\
\hline $\mathrm{JCl} 010$ & $\begin{array}{l}\text { A516 } \\
\text { Grade } 55\end{array}$ & 8760 & 51.409 & 2.9353 & 72.64 & $60 \mathrm{C}$ SDW AQUEOUS PHASE \\
\hline JCl 011 & $\begin{array}{l}\text { A516 } \\
\text { Grade } 55\end{array}$ & 8760 & 51.614 & 2.9572 & 72.89 & $60 \mathrm{C}$ SDW AQUEOUS PHASE \\
\hline $\mathrm{JCl} 012$ & $\begin{array}{l}\text { A516 } \\
\text { Grade } 55\end{array}$ & 8760 & 52.617 & 4.4224 & 106.93 & $60 \mathrm{C}$ SDW AQUEOUS PHASE \\
\hline KCA 007 & $\begin{array}{l}\text { A27 Grade } \\
70-40\end{array}$ & 8760 & 51.835 & 1.5936 & 39.31 & $60 \mathrm{C}$ SDW VAPOR PHASE \\
\hline KCA 008 & $\begin{array}{l}\text { A27 Grade } \\
70-40\end{array}$ & 8760 & 51.669 & 1.4780 & 36.58 & $60 \mathrm{C}$ SDW VAPOR PHASE \\
\hline KCA 009 & $\begin{array}{l}\text { A27 Grade } \\
70-40\end{array}$ & 8760 & 51.609 & 1.5809 & 39.17 & $60 \mathrm{C}$ SDW VAPOR PHASE \\
\hline KCA 010 & $\begin{array}{l}\text { A27 Grade } \\
70-40\end{array}$ & 8760 & 51.975 & 3.0283 & 74.51 & GOC SDW AQUEOUS PHASE \\
\hline KCA 011 & $\begin{array}{l}\text { A27 Grade } \\
70-40\end{array}$ & 8760 & 51.720 & 3.0173 & 74.60 & $60 \mathrm{C}$ SDW AQUEOUS PHASE \\
\hline KCA 012 & $\begin{array}{l}\text { A27 Grade } \\
70-40\end{array}$ & 8760 & 51.841 & 3.0572 & 75.41 & $60 \mathrm{C}$ SDW AQUEOUS PHASE \\
\hline $\mathrm{KCl} 007$ & $\begin{array}{l}\text { A27 Grade } \\
70-40\end{array}$ & 8760 & 50.546 & 1.5673 & 39.65 & $60 \mathrm{C}$ SDW VAPOR PHASE \\
\hline $\mathrm{KCl} 008$ & $\begin{array}{l}\text { A27 Grade } \\
70-40\end{array}$ & 8760 & 52.436 & 1.6007 & 39.04 & 60C SDW VAPOR PHASE \\
\hline $\mathrm{KCl} 009$ & $\begin{array}{l}\text { A27 Grade } \\
70-40\end{array}$ & 8760 & 51.299 & 1.4713 & 36.68 & $60 \mathrm{C}$ SDW VAPOR PHASE \\
\hline $\mathrm{KCl} 010$ & $\begin{array}{l}\text { A27 Grade } \\
70-40\end{array}$ & 8760 & 51.489 & 2.9077 & 72.21 & $60 \mathrm{C}$ SDW AQUEOUS PHASE \\
\hline KCl 011 & $\begin{array}{l}\text { A27 Grade } \\
70-40\end{array}$ & 8760 & 52.052 & 3.2916 & 80.87 & $60 \mathrm{C}$ SDW AQUEOUS PHASE \\
\hline $\mathrm{KCl} 012$ & $\begin{array}{l}\text { A27 Grade } \\
70-40\end{array}$ & 8760 & 51.780 & 3.4692 & 85.68 & $60 \mathrm{C}$ SDW AQUEOUS PHASE \\
\hline
\end{tabular}




\section{Corrosion Rate Calculations for Crevice Specimens}

For Rack Number : 22-1

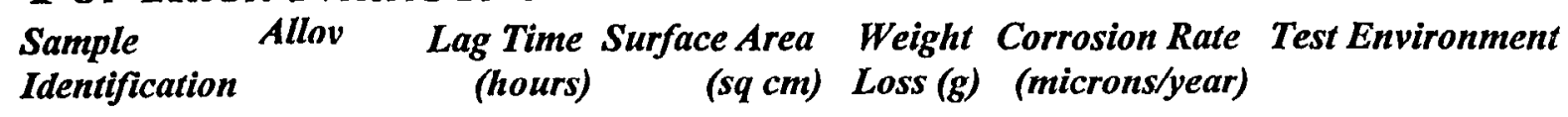

\begin{tabular}{|c|c|c|c|c|c|c|}
\hline ICA 031 & $\begin{array}{l}\text { A387 } \\
\text { Grade } 22\end{array}$ & 4632 & 53.186 & 0.6025 & 27.26 & $90 \mathrm{C}$ SDW VAPOR PHASE \\
\hline ICA 032 & $\begin{array}{l}\text { A387 } \\
\text { Grade } 22\end{array}$ & 4632 & 52.950 & 0.4919 & 22.35 & 90 C SDW VAPOR PHASE \\
\hline ICA 033 & $\begin{array}{l}\text { A387 } \\
\text { Grade } 22\end{array}$ & 4632 & 53.059 & 0.3954 & 17.93 & $90 \mathrm{C}$ SDW VAPOR PHASE \\
\hline ICA 034 & $\begin{array}{l}\text { A387 } \\
\text { Grade } 22\end{array}$ & 4632 & 52.461 & 1.9657 & 90.16 & $90 \mathrm{C}$ SDW AQUEOUS PHASE \\
\hline ICA 035 & $\begin{array}{l}\text { A387 } \\
\text { Grade } 22\end{array}$ & 4632 & 53.172 & 1.9743 & 89.34 & $90 \mathrm{C}$ SDW AQUEOUS PHASE \\
\hline ICA 036 & $\begin{array}{l}\text { A387 } \\
\text { Grade } 22\end{array}$ & 4632 & 53.174 & 2.1206 & 95.96 & $90 \mathrm{C}$ SDW AQUEOUS PHASE \\
\hline $\mathrm{ICH} 031$ & $\begin{array}{l}\text { A387 } \\
\text { Grade } 22\end{array}$ & 4632 & 53.026 & 0.4093 & 18.57 & $90 \mathrm{C}$ SDW VAPOR PHASE \\
\hline ICH 032 & $\begin{array}{l}\text { A387 } \\
\text { Grade } 22\end{array}$ & 4632 & 52.975 & 0.3557 & 16.16 & $90 \mathrm{C}$ SDW VAPOR PHASE \\
\hline $\mathrm{ICH} 033$ & $\begin{array}{l}\text { A387 } \\
\text { Grade } 22\end{array}$ & 4632 & 52.547 & 0.8031 & 36.77 & $90 \mathrm{C}$ SDW VAPOR PHASE \\
\hline ICH 034 & $\begin{array}{l}\text { A387 } \\
\text { Grade } 22\end{array}$ & 4632 & 52.312 & 2.1534 & 99.05 & $90 \mathrm{C}$ SDW AQUEOUS PHASE \\
\hline $\mathrm{ICH} 035$ & $\begin{array}{l}\text { A387 } \\
\text { Grade } 22\end{array}$ & 4632 & 52.012 & 1.2662 & 58.57 & $90 \mathrm{C}$ SDW AQUEOUS PHASE \\
\hline ICH 036 & $\begin{array}{l}\text { A387 } \\
\text { Grade } 22\end{array}$ & 4632 & 52.325 & 3.1047 & 142.77 & $90 \mathrm{C}$ SDW AQUEOUS PHASE \\
\hline SCA 031 & $\begin{array}{l}\text { A516 } \\
\text { Grade } 65\end{array}$ & 4632 & 53.131 & 2.9867 & 135.26 & $90 \mathrm{C}$ SDW VAPOR PHASE \\
\hline JCA 032 & $\begin{array}{l}\text { A516 } \\
\text { Grade } 55\end{array}$ & 4632 & 53.341 & 2.8839 & 130.09 & $90 \mathrm{C}$ SDW VAPOR PHASE \\
\hline JCA 033 & $\begin{array}{l}\text { A516 } \\
\text { Grade } 55\end{array}$ & 4632 & 53.674 & 2.0111 & 90.15 & $90 \mathrm{C}$ SDW VAPOR PHASE \\
\hline JCA 034 & $\begin{array}{l}\text { A516 } \\
\text { Grade } 55\end{array}$ & 4632 & 53.648 & 1.7160 & 76.96 & $90 \mathrm{C}$ SDW AQUEOUS PHASE \\
\hline JCA 035 & $\begin{array}{l}\text { A516 } \\
\text { Grade } 55\end{array}$ & 4632 & 52.808 & 1.5462 & 70.45 & $90 \mathrm{C}$ SDW AQUEOUS PHASE \\
\hline JCA 036 & $\begin{array}{l}\text { A516 } \\
\text { Grade } 55\end{array}$ & 4632 & 53.007 & 1.6367 & 74.29 & $90 \mathrm{C}$ SDW AQUEOUS PHASE \\
\hline JCI 031 & $\begin{array}{l}\text { A516 } \\
\text { Grade } 55\end{array}$ & 4632 & 52.570 & 1.3978 & 63.98 & $90 \mathrm{C}$ SDW VAPOR PHASE \\
\hline
\end{tabular}




\section{Corrosion Rate Calculations for Crevice Specimens}

For Rack Number : 22-1

$\begin{array}{llll}\text { Sample Allov Lag Time Surface Area Weight Corrosion Rate Test Environment } \\ \text { Identification } & \text { (hours) } & \text { (sq cm) Loss (g) (microns/year) }\end{array}$

\begin{tabular}{|c|c|c|c|c|c|c|}
\hline $\mathrm{JCl} 032$ & $\begin{array}{l}\text { A516 } \\
\text { Grade } 55\end{array}$ & 4632 & 51.667 & 1.3903 & 64.74 & 90 C SDW VAPOR PHASE \\
\hline $\mathrm{JCl} 033$ & $\begin{array}{l}\text { A516 } \\
\text { Grade } 55\end{array}$ & 4632 & 52.450 & 2.0873 & 95.75 & $90 \mathrm{C}$ SDW VAPOR PHASE \\
\hline $\mathrm{JCl} 034$ & $\begin{array}{l}\text { A516 } \\
\text { Grade } 55\end{array}$ & 4632 & 51.696 & 3.8763 & 180.42 & $90 \mathrm{C}$ SDW AQUEOUS PHASE \\
\hline $\mathrm{JCl} 035$ & $\begin{array}{l}\text { A516 } \\
\text { Grade } 55\end{array}$ & 4632 & 51.926 & 1.2534 & 58.08 & 90C SDW AQUEOUS PHASE \\
\hline $\mathrm{JCl} 036$ & $\begin{array}{l}\text { A516 } \\
\text { Grade } 55\end{array}$ & 4632 & 52.559 & 1.4476 & 66.27 & $90 \mathrm{C}$ SDW AQUEOUS PHASE \\
\hline KCA 031 & $\begin{array}{l}\text { A27 Grade } \\
70-40\end{array}$ & 4632 & 52.307 & 2.1361 & 98.76 & $90 \mathrm{C}$ SDW VAPOR PHASE \\
\hline KCA 032 & $\begin{array}{l}\text { A27 Grade } \\
70-40\end{array}$ & 4632 & 51.945 & 1.9102 & 88.93 & $90 \mathrm{C}$ SDW VAPOR PHASE \\
\hline KCA 033 & $\begin{array}{l}\text { A27 Grade } \\
70-40\end{array}$ & 4632 & 51.685 & 1.3464 & 63.00 & $90 \mathrm{C}$ SDW VAPOR PHASE \\
\hline KCA 034 & $\begin{array}{l}\text { A27 Grade } \\
70-40\end{array}$ & 4632 & 51.908 & 1.6924 & 78.85 & $90 \mathrm{C}$ SDW AQUEOUS PHASE \\
\hline KCA 035 & $\begin{array}{l}\text { A27 Grade } \\
70-40\end{array}$ & 4632 & 51.961 & 1.8275 & 85.06 & $90 \mathrm{C}$ SDW AQUEOUS PHASE \\
\hline KCA 036 & $\begin{array}{l}\text { A27 Grade } \\
70-40\end{array}$ & 4632 & 51.937 & 1.7086 & 79.56 & $90 \mathrm{C}$ SDW AQUEOUS PHASE \\
\hline KCl 031 & $\begin{array}{l}\text { A27 Grade } \\
70-40\end{array}$ & 4632 & 52.263 & 1.5021 & 69.51 & 90 C SDW VAPOR PHASE \\
\hline KCl 032 & $\begin{array}{l}\text { A27 Grade } \\
70-40\end{array}$ & 4632 & 50.374 & 1.0963 & 52.63 & $90 \mathrm{C}$ SDW VAPOR PHASE \\
\hline $\mathrm{KCl} 033$ & $\begin{array}{l}\text { A27 Grade } \\
70-40\end{array}$ & 4632 & 52.511 & 0.9677 & 44.57 & $90 \mathrm{C}$ SDW VAPOR PHASE \\
\hline KCl 034 & $\begin{array}{l}\text { A27 Grade } \\
70-40\end{array}$ & 4632 & 51.632 & 1.5919 & 74.56 & $90 \mathrm{C}$ SDW AQUEOUS PHASE \\
\hline $\mathrm{KCl} 035$ & $\begin{array}{l}\text { A27 Grade } \\
70-40\end{array}$ & 4632 & 52.097 & 1.6205 & 75.23 & $90 \mathrm{C}$ SDW AQUEOUS PHASE \\
\hline $\mathrm{KCl} 036$ & $\begin{array}{l}\text { A27 Grade } \\
70-40\end{array}$ & 4632 & 52.302 & 1.6664 & 77.05 & $90 \mathrm{C}$ SDW AQUEOUS PHASE \\
\hline
\end{tabular}




\section{Corrosion Rate Calculations for Crevice Specimens}

\section{For Rack Number : 22-2}

Sample Allov Lag Time Surface Area Weight Corrosion Rate Test Environment Identification (hours) (sq cm) Loss (g) (microns/year)

\begin{tabular}{|c|c|c|c|c|c|c|}
\hline ICA 037 & $\begin{array}{l}\text { A387 } \\
\text { Grade } 22\end{array}$ & 8832 & 53.134 & 1.0401 & 24.70 & $90 \mathrm{C}$ SDW VAPOR PHASE \\
\hline ICA 038 & $\begin{array}{l}\text { A387 } \\
\text { Grade } 22\end{array}$ & 8832 & 53.119 & 0.6884 & 16.35 & $90 \mathrm{C}$ SDW VAPOR PHASE \\
\hline ICA 039 & $\begin{array}{l}\text { A387 } \\
\text { Grade } 22\end{array}$ & 8832 & 53.283 & 0.7931 & 18.78 & $90 \mathrm{C}$ SDW VAPOR PHASE \\
\hline ICA 040 & $\begin{array}{l}\text { A387 } \\
\text { Grade } 22\end{array}$ & 8832 & 53.238 & 2.6970 & 63.93 & $90 \mathrm{C}$ SDW AQUEOUS PHASE \\
\hline ICA 041 & $\begin{array}{l}\text { A387 } \\
\text { Grade } 22\end{array}$ & 8832 & 53.019 & 2.1697 & 51.64 & $90 \mathrm{C}$ SDW AQUEOUS PHASE \\
\hline ICA 042 & $\begin{array}{l}\text { A387 } \\
\text { Grade } 22\end{array}$ & 8832 & 53.172 & 3.0014 & 71.23 & $90 \mathrm{C}$ SDW AQUEOUS PHASE \\
\hline ICH 037 & $\begin{array}{l}\text { A387 } \\
\text { Grade } 22\end{array}$ & 8832 & 52.197 & 1.0520 & 25.43 & $90 \mathrm{C}$ SDW VAPOR PHASE \\
\hline $\mathrm{ICH} 038$ & $\begin{array}{l}\text { A387 } \\
\text { Grade } 22\end{array}$ & 8832 & 52.327 & 1.4243 & 34.35 & 90 C SDW VAPOR PHASE \\
\hline ICH 039 & $\begin{array}{l}\text { A387 } \\
\text { Grade } 22\end{array}$ & 8832 & 52.834 & 2.4344 & 58.14 & $90 \mathrm{C}$ SDW VAPOR PHASE \\
\hline ICH 040 & $\begin{array}{l}\text { A387 } \\
\text { Grade } 22\end{array}$ & 8832 & 52.662 & 2.4830 & 59.50 & $90 \mathrm{C}$ SOW AQUEOUS PHASE \\
\hline ICH 041 & $\begin{array}{l}\text { A387 } \\
\text { Grade } 22\end{array}$ & 8832 & 52.648 & 3.5803 & 85.81 & $90 \mathrm{C}$ SDW AQUEOUS PHASE \\
\hline ICH 042 & $\begin{array}{l}\text { A387 } \\
\text { Grade } 22\end{array}$ & 8832 & 53.141 & 2.9301 & 69.58 & $90 \mathrm{C}$ SDW AQUEOUS PHASE \\
\hline JCA 037 & $\begin{array}{l}\text { A516 } \\
\text { Grade } 55\end{array}$ & 8832 & 52.730 & 2.6810 & 64.16 & $90 \mathrm{C}$ SDW VAPOR PHASE \\
\hline JCA 038 & $\begin{array}{l}\text { A516 } \\
\text { Grade } 55\end{array}$ & 8832 & 52.759 & 2.4686 & 59.04 & $90 \mathrm{C}$ SDW VAPOR PHASE \\
\hline JCA 039 & $\begin{array}{l}\text { A516 } \\
\text { Grade } 55\end{array}$ & 8832 & 53.158 & 2.1727 & 51.58 & 9O C SDW VAPOR PHASE \\
\hline JCA 040 & $\begin{array}{l}\text { A516 } \\
\text { Grade } 55\end{array}$ & 8832 & 52.764 & 1.7739 & 42.42 & $90 \mathrm{C}$ SDW AQUEOUS PHASE \\
\hline JCA 041 & $\begin{array}{l}\text { A516 } \\
\text { Grade } 55\end{array}$ & 8832 & 52.781 & 1.8203 & 45.91 & $90 \mathrm{C}$ SDW AQUEOUS PHASE \\
\hline JCA 042 & $\begin{array}{l}\text { A516 } \\
\text { Grade } 55\end{array}$ & 8832 & 53.250 & 1.2460 & 29.53 & $90 \mathrm{C}$ SDW AQUEOUS PHASE \\
\hline $\mathrm{JCl} 037$ & $\begin{array}{l}\text { A516 } \\
\text { Grade } 55\end{array}$ & 8832 & 52.695 & 2.3028 & 55.15 & $90 \mathrm{C}$ SDW VAPOR PHASE \\
\hline
\end{tabular}




\section{Corrosion Rate Calculations for Crevice Specimens}

\section{For Rack Number : 22-2}

Sample Allov Lag Time Surface Area Weight Corrosion Rate Test Environment

Identification

(hours) (sq cm) Loss (g) (microns/year)

\begin{tabular}{|c|c|c|c|c|c|c|}
\hline $\mathrm{JCl} 038$ & $\begin{array}{l}\text { A516 } \\
\text { Grade } 55\end{array}$ & 8832 & 51.872 & 2.7491 & 66.88 & $90 \mathrm{C}$ SDW VAPOR PHASE \\
\hline $\mathrm{JCl} 039$ & $\begin{array}{l}\text { A516 } \\
\text { Grade } 55\end{array}$ & 8832 & 51.749 & 3.0260 & 73.79 & $90 \mathrm{C}$ SDW VAPOR PHASE \\
\hline $\mathrm{JCl} 040$ & $\begin{array}{l}\text { A516 } \\
\text { Grade } 55\end{array}$ & 8832 & 52.131 & 2.6870 & 65.04 & $90 \mathrm{C}$ SDW AQUEOUS PHASE \\
\hline $\mathrm{JCl} 041$ & $\begin{array}{l}\text { A516 } \\
\text { Grade } 55\end{array}$ & 8832 & 51.723 & 2.6943 & 65.73 & $90 \mathrm{C}$ SDW AQUEOUS PHASE \\
\hline $\mathrm{JCl} 042$ & $\begin{array}{l}\text { A516 } \\
\text { Grade } 55\end{array}$ & 8832 & 51.735 & 3.6357 & 88.68 & $90 \mathrm{C}$ SDW AQUEOUS PHASE \\
\hline KCA 037 & $\begin{array}{l}\text { A27 Grade } \\
70-40\end{array}$ & 8832 & 51.348 & 1.8885 & 46.65 & $90 \mathrm{C}$ SDW VAPOR PHASE \\
\hline KCA 038 & $\begin{array}{l}\text { A27 Grade } \\
70-40\end{array}$ & 8832 & 52.111 & 2.1384 & 52.05 & 90 C SDW VAPOR PHASE \\
\hline KCA 039 & $\begin{array}{l}\text { A27 Grade } \\
70-40\end{array}$ & 8832 & 51.796 & 1.8046 & 44.19 & $90 \mathrm{C}$ SDW VAPOR PHASE \\
\hline KCA 040 & $\begin{array}{l}\text { A27 Grade } \\
70-40\end{array}$ & 8832 & 51.977 & 2.3967 & 58.48 & $90 \mathrm{C}$ SDW AQUEOUS PHASE \\
\hline KCA 041 & $\begin{array}{l}\text { A27 Grade } \\
70-40\end{array}$ & 8832 & 51.916 & 1.4684 & 35.87 & $90 \mathrm{C}$ SDW AQUEOUS PHASE \\
\hline KCA 042 & $\begin{array}{l}\text { A27 Grade } \\
70-40\end{array}$ & 8832 & 51.884 & 2.0512 & 50.14 & $90 \mathrm{C}$ SDW AQUEOUS PHASE \\
\hline KCl 037 & $\begin{array}{l}\text { A27 Grade } \\
70-40\end{array}$ & 8832 & 53.185 & 1.9821 & 47.27 & $90 \mathrm{C}$ SDW VAPOR PHASE \\
\hline KCl 038 & $\begin{array}{l}\text { A27 Grade } \\
70-40\end{array}$ & 8832 & 51.516 & 1.8171 & 44.74 & $90 \mathrm{C}$ SDW VAPOR PHASE \\
\hline KCl 039 & $\begin{array}{l}\text { A27 Grade } \\
70-40\end{array}$ & 8832 & 52.617 & 1.7186 & 41.43 & $90 \mathrm{C}$ SDW VAPOR PHASE \\
\hline $\mathrm{KCl} 040$ & $\begin{array}{l}\text { A27 Grade } \\
70-40\end{array}$ & 8832 & 52.475 & 2.0312 & 49.10 & $90 \mathrm{C}$ SDW AQUEOUS PHASE \\
\hline KCl 041 & $\begin{array}{l}\text { A27 Grade } \\
70-40\end{array}$ & 8832 & 52.222 & 2.2224 & 53.98 & $90 \mathrm{C}$ SDW AQUEOUS PHASE \\
\hline KCl 042 & $\begin{array}{l}\text { A27 Grade } \\
70-40\end{array}$ & 8832 & 52.294 & 1.6682 & 40.46 & $90 \mathrm{C}$ SDW AQUEOUS PHASE \\
\hline
\end{tabular}




\section{Corrosion Rate Calculations for Crevice Specimens}

For Rack Number : 21-1

$\begin{array}{lcrrr}\text { Sample } & \text { Allov } & \text { Lag Time Surface Area Weight Corrosion Rate Test Environment } \\ \text { Identification } & \text { (hours) } & \text { (sq cm) } & \text { Loss (g) (microns/year) }\end{array}$

\begin{tabular}{|c|c|c|c|c|c|c|}
\hline ICA 061 & $\begin{array}{l}\text { A387 } \\
\text { Grade } 22\end{array}$ & 4392 & 53.340 & 1.1379 & 54.13 & $60 \mathrm{C}$ SCW VAPOR PHASE \\
\hline ICA 062 & $\begin{array}{l}\text { A387 } \\
\text { Grade } 22\end{array}$ & 4392 & 52.912 & 1.2126 & 58.15 & $60 \mathrm{C}$ SCW VAPOR PHASE \\
\hline ICA 063 & $\begin{array}{l}\text { A387 } \\
\text { Grade } 22\end{array}$ & 4392 & 53.435 & 1.2510 & 59.41 & $60 \mathrm{C}$ SCW VAPOR PHASE \\
\hline ICA 064 & $\begin{array}{l}\text { A387 } \\
\text { Grade } 22\end{array}$ & 4392 & 53.053 & 0.4566 & 21.84 & $60 \mathrm{C}$ SCW AQUEOUS PHASE \\
\hline ICA 065 & $\begin{array}{l}\text { A387 } \\
\text { Grade } 22\end{array}$ & 4392 & 52.614 & 0.4204 & 20.28 & $60 \mathrm{C} \mathrm{SCW}$ AQUEOUS PHASE \\
\hline ICA 066 & $\begin{array}{l}\text { A387 } \\
\text { Grade } 22\end{array}$ & 4392 & 53.300 & 0.4010 & 19.09 & $60 \mathrm{C}$ SCW AQUEOUS PHASE \\
\hline ICH 061 & $\begin{array}{l}\text { A387 } \\
\text { Grade } 22\end{array}$ & 4392 & 52.958 & 1.5668 & 75.08 & $60 \mathrm{C} \mathrm{SCW}$ VAPOR PHASE \\
\hline $1 \mathrm{CH} 062$ & $\begin{array}{l}\text { A387 } \\
\text { Grade } 22\end{array}$ & 4392 & 52.972 & 2.0224 & 96.88 & $60 \mathrm{C}$ SCW VAPOR PHASE \\
\hline ICH 063 & $\begin{array}{l}\text { A387 } \\
\text { Grade } 22\end{array}$ & 4392 & 53.075 & 3.7594 & 179.74 & $60 \mathrm{C}$ SCW VAPOR PHASE \\
\hline $\mathrm{ICH} 064$ & $\begin{array}{l}\text { A387 } \\
\text { Grade } 22\end{array}$ & 4392 & 52,879 & 0.5769 & 27.68 & $60 \mathrm{C}$ SCW AQUEOUS PHASE \\
\hline ICH 065 & $\begin{array}{l}\text { A387 } \\
\text { Grade } 22\end{array}$ & 4392 & 52.118 & 0.5883 & 28.64 & $60 \mathrm{C}$ SCW AQUEOUS PHASE \\
\hline ICH 066 & $\begin{array}{l}\text { A387 } \\
\text { Grade } 22\end{array}$ & 4392 & 52.436 & 0.6079 & 29.42 & $60 \mathrm{C}$ SCW AQUEOUS PHASE \\
\hline JCA 061 & $\begin{array}{l}\text { A516 } \\
\text { Grade } 55\end{array}$ & 4392 & 53.403 & 2.5857 & 122.87 & $60 \mathrm{C}$ SCW VAPOR PHASE \\
\hline JCA 062 & $\begin{array}{l}\text { A516 } \\
\text { Grade } 55\end{array}$ & 4392 & $\mathbf{5 3 . 4 9 5}$ & 3.4959 & 165.83 & $60 \mathrm{C}$ SCW VAPOR PHASE \\
\hline JCA 063 & $\begin{array}{l}\text { A516 } \\
\text { Grade } 55\end{array}$ & 4392 & 53.363 & 4.4477 & 211.50 & $60 \mathrm{C}$ SCW VAPOR PHASE \\
\hline JCA 064 & $\begin{array}{l}\text { A516 } \\
\text { Grade } 55\end{array}$ & 4392 & 53.222 & 1.3672 & 65.19 & $60 \mathrm{C}$ SCW AQUEOUS PHASE \\
\hline JCA 065 & $\begin{array}{l}\text { A516 } \\
\text { Grade } 55\end{array}$ & 4392 & 52.514 & 1.3518 & 65.32 & $60 \mathrm{C}$ SCW AQUEOUS PHASE \\
\hline JCA 066 & $\begin{array}{l}\text { A516 } \\
\text { Grade } 55\end{array}$ & 4392 & 53.049 & 1.3174 & 63.02 & $60 \mathrm{C}$ SCW AQUEOUS PHASE \\
\hline JCl 061 & $\begin{array}{l}\text { A516 } \\
\text { Grade } 55\end{array}$ & 4392 & 51.656 & 4.2432 & 208.44 & $60 \mathrm{C}$ SCW VAPOR PHASE \\
\hline
\end{tabular}




\section{Corrosion Rate Calculations for Crevice Specimens}

\section{For Rack Number : 21-1}

Sample Allov Lag Time Surface Area Weight Corrosion Rate Test Environment Identification (hours) (sq cm) Loss (g) (microns/year)

\begin{tabular}{|c|c|c|c|c|c|c|}
\hline JCl 062 & $\begin{array}{l}\text { A516 } \\
\text { Grade } 55\end{array}$ & 4392 & 51.792 & 4.7602 & 233.23 & $60 \mathrm{C}$ SCW VAPOR PHASE \\
\hline $\mathrm{JCl} 063$ & $\begin{array}{l}\text { A516 } \\
\text { Grade } 55\end{array}$ & 4392 & 51.780 & 5.2537 & 257.47 & $60 \mathrm{C}$ SCW VAPOR PHASE \\
\hline $\mathrm{JCl} 064$ & $\begin{array}{l}\text { A516 } \\
\text { Grade } 55\end{array}$ & 4392 & 51.806 & 1.1847 & 58.03 & $60 \mathrm{C}$ SCW AQUEOUS PHASE \\
\hline JCl 065 & $\begin{array}{l}\text { A516 } \\
\text { Grade } 55\end{array}$ & 4392 & 51.573 & 1.2228 & 60.17 & $60 \mathrm{C}$ SCW AQUEOUS PHASE \\
\hline $\mathrm{JCl} 066$ & $\begin{array}{l}\text { A516 } \\
\text { Grade } 55\end{array}$ & 4392 & 52.472 & 1.2927 & 62.52 & $60 \mathrm{C}$ SCW AQUEOUS PHASE \\
\hline KCA 061 & $\begin{array}{l}\text { A27 Grade } \\
70-40\end{array}$ & 4392 & 51.789 & 2.2727 & 111.93 & $60 \mathrm{C}$ SCW VAPOR PHASE \\
\hline KCA 062 & $\begin{array}{l}\text { A27 Grade } \\
70-40\end{array}$ & 4392 & 51.652 & 2.2598 & 111.59 & $60 \mathrm{C}$ SCW VAPOR PHASE \\
\hline KCA 063 & $\begin{array}{l}\text { A27 Grade } \\
70-40\end{array}$ & 4392 & 51.648 & 2.3443 & 115.77 & $60 \mathrm{C}$ SCW VAPOR PHASE \\
\hline KCA 064 & $\begin{array}{l}\text { A27 Grade } \\
70-40\end{array}$ & 4392 & 51.885 & 1.2545 & 61.67 & $60 \mathrm{C}$ SCW AQUEOUS PHASE \\
\hline KCA 065 & $\begin{array}{l}\text { A27 Grade } \\
70-40\end{array}$ & 4392 & 51.765 & 1.2354 & 60.87 & $60 \mathrm{C}$ SCW AQUEOUS PHASE \\
\hline KCA 066 & $\begin{array}{l}\text { A27 Grade } \\
70-40\end{array}$ & 4392 & 51.629 & 1.2578 & 62.14 & $60 \mathrm{C}$ SCW AQUEOUS PHASE \\
\hline KCl 061 & $\begin{array}{l}\text { A27 Grade } \\
70-40\end{array}$ & 4392 & 52.406 & 3.5040 & 170.54 & $60 \mathrm{C}$ SCW VAPOR PHASE \\
\hline KCl 062 & $\begin{array}{l}\text { A27 Grade } \\
70-40\end{array}$ & 4392 & 51.640 & 3.6310 & 179.34 & $60 \mathrm{C}$ SCW VAPOR PHASE \\
\hline $\mathrm{KCl} 063$ & $\begin{array}{l}\text { A27 Grade } \\
70-40\end{array}$ & 4392 & 52.357 & 5.1544 & 251.10 & $60 \mathrm{C}$ SCW VAPOR PHASE \\
\hline KCl 064 & $\begin{array}{l}\text { A27 Grade } \\
70-40\end{array}$ & 4392 & 52.117 & 1.7984 & 88.01 & $60 \mathrm{C}$ SCW AQUEOUS PHASE \\
\hline KCI 065 & $\begin{array}{l}\text { A27 Grade } \\
70-40\end{array}$ & 4392 & 52.148 & 2.1304 & 104.20 & $60 \mathrm{C}$ SCW AQUEOUS PHASE \\
\hline $\mathrm{KCl} 066$ & $\begin{array}{l}\text { A27 Grade } \\
70-40\end{array}$ & 4392 & 51.831 & 1.7999 & 88.57 & $60 \mathrm{C}$ SCW AQUEOUS PHASE \\
\hline
\end{tabular}




\section{Corrosion Rate Calculations for Crevice Specimens}

For Rack Number : 21-2

Sample Allov Lag Time Surface Area Weight Corrosion Rate Test Environment Identification (hours) (sq cm) Loss (g) (microns/year)

\begin{tabular}{|c|c|c|c|c|c|c|}
\hline ICA 067 & $\begin{array}{l}\text { A387 } \\
\text { Grade } 22\end{array}$ & 8760 & 53.003 & 2.0071 & 48.18 & $60 \mathrm{C}$ SCW VAPOR PHASE \\
\hline ICA 068 & $\begin{array}{l}\text { A387 } \\
\text { Grade } 22\end{array}$ & 8760 & 53.147 & 2.6897 & 64.39 & $60 \mathrm{C}$ SCW VAPOR PHASE \\
\hline ICA 069 & $\begin{array}{l}\text { A387 } \\
\text { Grade } 22\end{array}$ & 8760 & 53.148 & 4.8859 & 116.96 & $60 \mathrm{C}$ SCW VAPOR PHASE \\
\hline ICA 070 & $\begin{array}{l}\text { A387 } \\
\text { Grade } 22\end{array}$ & 8760 & 52.515 & 0.2117 & 5.13 & $60 \mathrm{C}$ SCW AQUEOUS PHASE \\
\hline ICA 071 & $\begin{array}{l}\text { A387 } \\
\text { Grade } 22\end{array}$ & 8760 & 53.266 & 0.1924 & 4.60 & $60 \mathrm{C}$ SCW AQUEOUS PHASE \\
\hline ICA 072 & $\begin{array}{l}\text { A387 } \\
\text { Grade } 22\end{array}$ & 8760 & 52.617 & 0.3660 & 8.85 & $60 \mathrm{C}$ SCW AQUEOUS PHASE \\
\hline ICH 067 & $\begin{array}{l}\text { A387 } \\
\text { Grade } 22\end{array}$ & 8760 & 52.057 & 5.9884 & 146.36 & $60 \mathrm{C}$ SCW VAPOR PHASE \\
\hline 1CH 068 & $\begin{array}{l}\text { A387 } \\
\text { Grade } 22\end{array}$ & 8760 & 52.500 & 7.3001 & 176.91 & $60 \mathrm{C} \mathrm{SCW}$ VAPOR PHASE \\
\hline ICH 069 & $\begin{array}{l}\text { A387 } \\
\text { Grade } 22\end{array}$ & 8760 & 52.372 & 8.3929 & 203.89 & $60 \mathrm{C}$ SCW VAPOR PHASE \\
\hline ICH 070 & $\begin{array}{l}\text { A387 } \\
\text { Grade } 22\end{array}$ & 8760 & 52.499 & 0.3598 & 8.72 & $60 \mathrm{C} \mathrm{SCW}$ AQUEOUS PHASE \\
\hline ICH 071 & $\begin{array}{l}\text { A387 } \\
\text { Grade } 22\end{array}$ & 8760 & 52.756 & 0.3466 & 8.36 & $60 \mathrm{C}$ SCW AQUEOUS PHASE \\
\hline $\mathrm{ICH} 072$ & $\begin{array}{l}\text { A387 } \\
\text { Grade } 22\end{array}$ & 8760 & 52.866 & 0.3402 & 8.19 & $60 \mathrm{C}$ SCW AQUEOUS PHASE \\
\hline JCA 067 & $\begin{array}{l}\text { A516 } \\
\text { Grade } 55\end{array}$ & 8760 & 53.173 & 4.5428 & 108.70 & $60 \mathrm{C} \mathrm{SCW}$ VAPOR PHASE \\
\hline JCA 068 & $\begin{array}{l}\text { A516 } \\
\text { Grade } 55\end{array}$ & 8760 & 52.561 & 5.3039 & 128.38 & $60 \mathrm{C}$ SCW VAPOR PHASE \\
\hline JCA 069 & $\begin{array}{l}\text { A516 } \\
\text { Grade } 55\end{array}$ & 8760 & 53.109 & 6.1912 & 148.32 & $60 \mathrm{C}$ SCW VAPOR PHASE \\
\hline JCA 070 & $\begin{array}{l}\text { A516 } \\
\text { Grade } 55\end{array}$ & 8760 & 52.708 & 0.2804 & 6.77 & $60 \mathrm{C} \mathrm{SCW}$ AQUEOUS PHASE \\
\hline JCA 071 & $\begin{array}{l}\text { A516 } \\
\text { Grade } 55\end{array}$ & 8760 & 53.063 & 0.4198 & 10.07 & $60 \mathrm{C}$ SCW AQUEOUS PHASE \\
\hline JCA 072 & $\begin{array}{l}\text { A516 } \\
\text { Grade } 55\end{array}$ & 8760 & 53.862 & 0.4961 & 11.72 & $60 \mathrm{C}$ SCW AQUEOUS PHASE \\
\hline $\mathrm{JCl} 067$ & $\begin{array}{l}\text { A516 } \\
\text { Grade } 55\end{array}$ & 8760 & 52.418 & 7.1119 & 172.62 & $60 \mathrm{C}$ SCW VAPOR PHASE \\
\hline
\end{tabular}




\section{Corrosion Rate Calculations for Crevice Specimens}

For Rack Number : 21-2

$\begin{array}{llll}\text { Sample Allov } & \text { Lag Time Surface Area Weight Corrosion Rate Test Environment } \\ \text { Identification } & \text { (hours) } & \text { (sq cm) } & \text { Loss }(g) \text { (microns/year) }\end{array}$

\begin{tabular}{|c|c|c|c|c|c|c|}
\hline $\mathrm{sCl} 068$ & $\begin{array}{l}\text { A516 } \\
\text { Grade } 55\end{array}$ & 8760 & 51.791 & 8.3212 & 204.41 & $60 \mathrm{C}$ SCW VAPOR PHASE \\
\hline JCl 069 & $\begin{array}{l}\text { A516 } \\
\text { Grade } 55\end{array}$ & 8760 & 51.723 & 10.8980 & 268.07 & $60 \mathrm{C}$ SCW VAPOR PHASE \\
\hline $\mathrm{JCl} 070$ & $\begin{array}{l}\text { A516 } \\
\text { Grade } 55\end{array}$ & 8760 & 51.616 & 0.4949 & 12.20 & $60 \mathrm{C}$ SCW AQUEOUS PHASE \\
\hline JCI 071 & $\begin{array}{l}\text { A516 } \\
\text { Grade } 55\end{array}$ & 8760 & 51.548 & 0.5228 & 12.90 & $60 \mathrm{C}$ SCW AQUEOUS PHASE \\
\hline sCl 072 & $\begin{array}{l}\text { A516 } \\
\text { Grade } 55\end{array}$ & 8760 & 52.813 & 0.5393 & 12.99 & $60 \mathrm{C}$ SCW AQUEOUS PHASE \\
\hline KCA 067 & $\begin{array}{l}\text { A27 Grade } \\
70-40\end{array}$ & 8760 & 50.805 & 3.1889 & 80.27 & $60 \mathrm{C}$ SCW VAPOR PHASE \\
\hline KCA 068 & $\begin{array}{l}\text { A27 Grade } \\
70-40\end{array}$ & 8760 & 51.536 & 4.2533 & 105.54 & $60 \mathrm{C}$ SCW VAPOR PHASE \\
\hline KCA 069 & $\begin{array}{l}\text { A27 Grade } \\
70-40\end{array}$ & 8760 & 51.626 & 4.9141 & 121.72 & $60 \mathrm{C}$ SCW VAPOR PHASE \\
\hline KCA 071 & $\begin{array}{l}\text { A27 Grade } \\
70-40\end{array}$ & 8760 & 51.826 & 0.4899 & 12.09 & $60 \mathrm{C}$ SCW AQUEOUS PHASE \\
\hline KCA 072 & $\begin{array}{l}\text { A27 Grade } \\
70-40\end{array}$ & 8760 & 49.808 & 0.5594 & 14.36 & $60 \mathrm{C}$ SCW AQUEOUS PHASE \\
\hline KCl 067 & $\begin{array}{l}\text { A27 Grade } \\
70-40\end{array}$ & 8760 & 51.654 & 6.9146 & 171.18 & $60 \mathrm{C}$ SCW VAPOR PHASE \\
\hline $\mathrm{KCl} 068$ & $\begin{array}{l}\text { A27 Grade } \\
70-40\end{array}$ & 8760 & 52.718 & 6.6746 & 161.90 & $60 \mathrm{C}$ SCW VAPOR PHASE \\
\hline KCl 069 & $\begin{array}{l}\text { A27 Grade } \\
70-40\end{array}$ & 8760 & 52.646 & 7.3699 & 179.01 & $60 \mathrm{C}$ SCW VAPOR PHASE \\
\hline $\mathrm{KCl} 070$ & $\begin{array}{l}\text { A27 Grade } \\
70-40\end{array}$ & 8760 & 51.698 & 0.4937 & 12.21 & $60 \mathrm{C}$ SCW AQUEOUS PHASE \\
\hline KCl 071 & $\begin{array}{l}\text { A27 Grade } \\
70-40\end{array}$ & 8760 & 52.357 & 0.4889 & 11.94 & $60 \mathrm{C}$ SCW AQUEOUS PHASE \\
\hline KCl 072 & $\begin{array}{l}\text { A27 Grade } \\
70-40\end{array}$ & 8760 & 52.197 & 0.5152 & 12.62 & $60 \mathrm{C}$ SCW AQUEOUS PHASE \\
\hline
\end{tabular}




\section{Corrosion Rate Calculations for Crevice Specimens For Rack Number : 20-1}

Sample Allov Lag Time Surface Area Weight Corrosion Rate Test Environment Identification (hours) (sq cm) Loss (g) (microns/year)

\begin{tabular}{|c|c|c|c|c|c|c|}
\hline ICA 091 & $\begin{array}{l}\text { A387 } \\
\text { Grade } 22\end{array}$ & 4344 & 52.892 & 1.7901 & 86.83 & $90 \mathrm{C}$ SCW VAPOR PHASE \\
\hline ICA 092 & $\begin{array}{l}\text { A387 } \\
\text { Grade } 22\end{array}$ & 4344 & 53.192 & 2.7451 & 132.40 & $90 \mathrm{C}$ SCW VAPOR PHASE \\
\hline ICA 093 & $\begin{array}{l}\text { A387 } \\
\text { Grade } 22\end{array}$ & 4344 & 53.154 & 4.0261 & 194.33 & $90 \mathrm{C}$ SCW VAPOR PHASE \\
\hline ICA 094 & $\begin{array}{l}\text { A387 } \\
\text { Grade } 22\end{array}$ & 4344 & 53.147 & 0.1791 & 8.65 & $90 \mathrm{C}$ SCW AQUEOUS PHASE \\
\hline ICA 095 & $\begin{array}{l}\text { A387 } \\
\text { Grade } 22\end{array}$ & 4344 & 53.432 & 0.1997 & 9.59 & $90 \mathrm{C}$ SCW AQUEOUS PHASE \\
\hline ICA 096 & $\begin{array}{l}\text { A387 } \\
\text { Grade } 22\end{array}$ & 4344 & 53.193 & 0.2301 & 11.10 & 90 C SCW AQUEOUS PHASE \\
\hline ICH 091 & $\begin{array}{l}\text { A387 } \\
\text { Grade } 22\end{array}$ & 4344 & 52.961 & 5.2295 & 253.33 & $90 \mathrm{C}$ SCW VAPOR PHASE \\
\hline ICH 092 & $\begin{array}{l}\text { A387 } \\
\text { Grade } 22\end{array}$ & 4344 & 52.986 & 6.6431 & 321.66 & $90 \mathrm{C}$ SCW VAPOR PHASE \\
\hline ICH 093 & $\begin{array}{l}\text { A387 } \\
\text { Grade } 22\end{array}$ & 4344 & 52.410 & 9.2181 & 451.26 & $90 \mathrm{C}$ SCW VAPOR PHASE \\
\hline $\mathrm{ICHO} 094$ & $\begin{array}{l}\text { A387 } \\
\text { Grade } 22\end{array}$ & 4344 & 52.761 & 0.1791 & 8.71 & 90 C SCW AQUEOUS PHASE \\
\hline ICH 095 & $\begin{array}{l}\text { A387 } \\
\text { Grade } 22\end{array}$ & 4344 & 52.742 & 0.1718 & 8.36 & $90 \mathrm{C}$ SCW AQUEOUS PHASE \\
\hline ICH 096 & $\begin{array}{l}\text { A387 } \\
\text { Grade } 22\end{array}$ & 4344 & 52.624 & 0.1764 & 8.60 & $90 \mathrm{C}$ SCW AQUEOUS PHASE \\
\hline JCA 091 & $\begin{array}{l}\text { A516 } \\
\text { Grade } 55\end{array}$ & 4344 & 52.876 & 3.8224 & 185.47 & 90 C SCW VAPOR PHASE \\
\hline JCA 092 & $\begin{array}{l}\text { A516 } \\
\text { Grade } 55\end{array}$ & 4344 & 53.685 & 4.7766 & 228.28 & $90 \mathrm{C}$ SCW VAPOR PHASE \\
\hline JCA 093 & $\begin{array}{l}\text { A516 } \\
\text { Grade } 55\end{array}$ & 4344 & 53.669 & 4.7703 & 228.04 & $90 \mathrm{C}$ SCW VAPOR PHASE \\
\hline JCA 094 & $\begin{array}{l}\text { A516 } \\
\text { Grade } 55\end{array}$ & 4344 & 53.110 & 0.2533 & 12.24 & $90 \mathrm{C}$ SCW AQUEOUS PHASE \\
\hline JCA 095 & $\begin{array}{l}\text { A516 } \\
\text { Grade } 55\end{array}$ & 4344 & 53.088 & 0.1665 & 8.05 & $90 \mathrm{C}$ SCW AQUEOUS PHASE \\
\hline JCA 096 & $\begin{array}{l}\text { A516 } \\
\text { Grade } 55\end{array}$ & 4344 & 52.733 & 0.1780 & 8.66 & $90 \mathrm{C}$ SCW AQUEOUS PHASE \\
\hline JCl 091 & $\begin{array}{l}\text { A516 } \\
\text { Grade } 55\end{array}$ & 4344 & 51.751 & 5.5776 & 276.51 & $90 \mathrm{C}$ SCW VAPOR PHASE \\
\hline
\end{tabular}




\section{Corrosion Rate Calculations for Crevice Specimens}

For Rack Number : 20-1

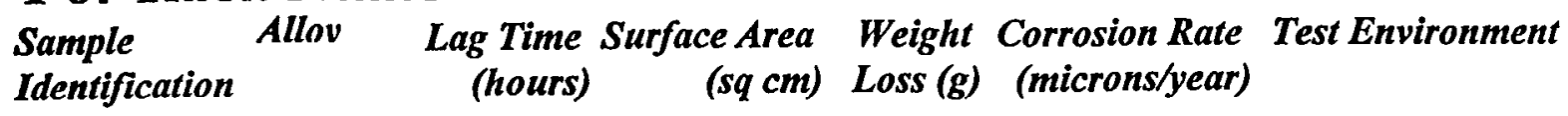

\begin{tabular}{|c|c|c|c|c|c|c|}
\hline JCl 092 & $\begin{array}{l}\text { A516 } \\
\text { Grade } 55\end{array}$ & 4344 & 51.847 & 3.5537 & 175.85 & $90 \mathrm{C}$ SCW VAPOR PHASE \\
\hline $\mathrm{JCl} 093$ & $\begin{array}{l}\text { A516 } \\
\text { Grade } 55\end{array}$ & 4344 & 52.750 & 5.5332 & 269.12 & $90 \mathrm{C}$ SCW VAPOR PHASE \\
\hline JCl 094 & $\begin{array}{l}\text { A516 } \\
\text { Grade } 55\end{array}$ & 4344 & 52.632 & 0.1516 & 7.39 & $90 \mathrm{C}$ SCW AQUEOUS PHASE \\
\hline JCI 095 & $\begin{array}{l}\text { A516 } \\
\text { Grade } 55\end{array}$ & 4344 & 51.547 & 0.1832 & 9.12 & $90 \mathrm{C}$ SCW AQUEOUS PHASE \\
\hline JCl 096 & $\begin{array}{l}\text { A516 } \\
\text { Grade } 55\end{array}$ & 4344 & 51.506 & 0.1811 & 9.02 & $90 \mathrm{C}$ SCW AQUEOUS PHASE \\
\hline KCA 091 & $\begin{array}{l}\text { A27 Grade } \\
70-40\end{array}$ & 4344 & 52.074 & 1.5204 & 75.29 & $90 \mathrm{C}$ SCW VAPOR PHASE \\
\hline KCA 092 & $\begin{array}{l}\text { A27 Grade } \\
70-40\end{array}$ & 4344 & 51.663 & 1.8697 & 93.33 & $90 \mathrm{C}$ SCW VAPOR PHASE \\
\hline KCA 093 & $\begin{array}{l}\text { A27 Grade } \\
70-40\end{array}$ & 4344 & 51.673 & 2.0501 & 102.31 & $90 \mathrm{C}$ SCW VAPOR PHASE \\
\hline KCA 094 & $\begin{array}{l}\text { A27 Grade } \\
70-40\end{array}$ & 4344 & 51.323 & 0.2941 & 14.78 & $90 \mathrm{C}$ SCW AQUEOUS PHASE \\
\hline KCA 095 & $\begin{array}{l}\text { A27 Grade } \\
70-40\end{array}$ & 4344 & 51.407 & 0.2997 & 15.03 & $90 \mathrm{C}$ SCW AQUEOUS PHASE \\
\hline KCA 096 & $\begin{array}{l}\text { A27 Grade } \\
70-40\end{array}$ & 4344 & 51.998 & 0.2541 & 12.60 & $90 \mathrm{C}$ SCW AQUEOUS PHASE \\
\hline KCl 091 & $\begin{array}{l}\text { A27 Grade } \\
70-40\end{array}$ & 4344 & 52.637 & 2.6357 & 129.12 & $90 \mathrm{C}$ SCW VAPOR PHASE \\
\hline $\mathrm{KCl} 092$ & $\begin{array}{l}\text { A27 Grade } \\
70-40\end{array}$ & 4344 & 51.868 & 3.6374 & 180.84 & $90 \mathrm{C}$ SCW VAPOR PHASE \\
\hline KCl 093 & $\begin{array}{l}\text { A27 Grade } \\
70-40\end{array}$ & 4344 & $\mathbf{5 2 . 3 2 0}$ & 4.4346 & 218.57 & $90 \mathrm{C}$ SCW VAPOR PHASE \\
\hline KCl 094 & $\begin{array}{l}\text { A27 Grade } \\
70-40\end{array}$ & 4344 & 52.657 & 0.1988 & 9.74 & $90 \mathrm{C}$ SCW AQUEOUS PHASE \\
\hline KCl 095 & $\begin{array}{l}\text { A27 Grade } \\
70-40\end{array}$ & 4344 & 51.202 & 0.2076 & 10.46 & $90 \mathrm{C}$ SCW AQUEOUS PHASE \\
\hline KCl 096 & $\begin{array}{l}\text { A27 Grade } \\
70-40\end{array}$ & 4344 & 51.991 & 0.2312 & 11.47 & $90 \mathrm{C}$ SCW AQUEOUS PHASE \\
\hline
\end{tabular}




\section{Corrosion Rate Calculations for Crevice Specimens}

\section{For Rack Number : 20-2}

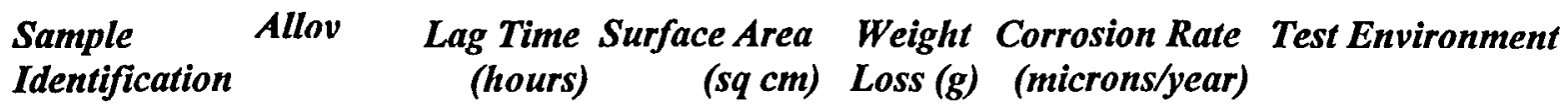

\begin{tabular}{|c|c|c|c|c|c|c|}
\hline ICA 097 & $\begin{array}{l}\text { A387 } \\
\text { Grade } 22\end{array}$ & 8736 & 53007 & 3.3840 & 81.44 & $90 \mathrm{C}$ SCW VAPOR PHASE \\
\hline ICA 098 & $\begin{array}{l}\text { A387 } \\
\text { Grade } 22\end{array}$ & 8736 & 53.089 & 5.8262 & 140.01 & $90 \mathrm{C}$ SCW VAPOR PHASE \\
\hline ICA 099 & $\begin{array}{l}\text { A387 } \\
\text { Grade } 22\end{array}$ & 8736 & 52.695 & 8.2831 & 200.53 & $90 \mathrm{C}$ SCW VAPOR PHASE \\
\hline ICA 100 & $\begin{array}{l}\text { A387 } \\
\text { Grade } 22\end{array}$ & 8736 & 52.905 & 0.1382 & 3.33 & $90 \mathrm{C}$ SCW AQUEOUS PHASE \\
\hline ICA 101 & $\begin{array}{l}\text { A387 } \\
\text { Grade } 22\end{array}$ & 8736 & 52.818 & 0.1401 & 3.38 & $90 \mathrm{C}$ SCW AQUEOUS PHASE \\
\hline ICA 102 & $\begin{array}{l}\text { A387 } \\
\text { Grade } 22\end{array}$ & 8736 & 52.146 & 0.1386 & 3.39 & $90 \mathrm{C}$ SCW AQUEOUS PHASE \\
\hline 1CH 097 & $\begin{array}{l}\text { A387 } \\
\text { Grade } 22\end{array}$ & 8736 & 52.987 & 11.4118 & 274.76 & $90 \mathrm{C}$ SCW VAPOR PHASE \\
\hline $\mathrm{ICH} 098$ & $\begin{array}{l}\text { A387 } \\
\text { Grade } 22\end{array}$ & 8736 & 52.742 & 11.5974 & 280.53 & $90 \mathrm{C}$ SCW VAPOR PHASE \\
\hline ICH 100 & $\begin{array}{l}\text { A387 } \\
\text { Grade } 22\end{array}$ & 8736 & 52.653 & 0.1297 & 3.14 & $90 \mathrm{C}$ SCW AQUEOUS PHASE \\
\hline ICH 101 & $\begin{array}{l}\text { A387 } \\
\text { Grade } 22\end{array}$ & 8736 & 52.481 & 0.1347 & 3.27 & $90 \mathrm{C} \mathrm{SCW}$ AQUEOUS PHASE \\
\hline ICH 102 & $\begin{array}{l}\text { A387 } \\
\text { Grade } 22\end{array}$ & 8736 & 52.588 & 0.1303 & 3.16 & $90 \mathrm{C}$ SCW AQUEOUS PHASE \\
\hline JCA 097 & $\begin{array}{l}\text { A516 } \\
\text { Grade } 55\end{array}$ & 8736 & 52.791 & 5.7757 & 139.58 & $90 \mathrm{C}$ SCW VAPOR PHASE \\
\hline JCA 098 & $\begin{array}{l}\text { A516 } \\
\text { Grade } 55\end{array}$ & 8736 & 53.626 & 5.6287 & 133.91 & $90 \mathrm{C}$ SCW VAPOR PHASE \\
\hline JCA 099 & $\begin{array}{l}\text { A516 } \\
\text { Grade } 55\end{array}$ & 8736 & 53.616 & 5.0981 & 121.31 & $90 \mathrm{C}$ SCW VAPOR PHASE \\
\hline JCA 100 & $\begin{array}{l}\text { A516 } \\
\text { Grade } 55\end{array}$ & 8736 & 53.079 & 0.3199 & 7.69 & $90 \mathrm{C}$ SCW AQUEOUS PHASE \\
\hline JCA 101 & $\begin{array}{l}\text { A516 } \\
\text { Grade } 55\end{array}$ & 8736 & 53.314 & 0.2728 & 6.53 & $90 \mathrm{C}$ SCW AQUEOUS PHASE \\
\hline JCA 102 & $\begin{array}{l}\text { A516 } \\
\text { Grade } 55\end{array}$ & 8736 & $\mathbf{5 2 . 9 2 6}$ & 0.2141 & 5.16 & $90 \mathrm{C}$ SCW AQUEOUS PHASE \\
\hline JCl 097 & $\begin{array}{l}\text { A516 } \\
\text { Grade } 55\end{array}$ & 8736 & 51.724 & 5.8566 & 144.45 & $90 \mathrm{C}$ SCW VAPOR PHASE \\
\hline $\mathrm{JCl} 098$ & $\begin{array}{l}\text { A516 } \\
\text { Grade } 55\end{array}$ & 8736 & 52.301 & 5.4115 & 132.00 & $90 \mathrm{C}$ SCW VAPOR PHASE \\
\hline
\end{tabular}




\section{Corrosion Rate Calculations for Crevice Specimens}

For Rack Number : 20-2

$\begin{array}{lccc}\text { Sample Allov } & \text { Lag Time Surface Area Weight Corrosion Rate Test Environment } \\ \text { Identification } & \text { (hours) } & \text { (sq cm) Loss }(\mathrm{g}) & \text { (microns/year) }\end{array}$

\begin{tabular}{|c|c|c|c|c|c|c|}
\hline JCl 099 & $\begin{array}{l}\text { A516 } \\
\text { Grade } 55\end{array}$ & 8736 & 51.818 & 5.9495 & 146.48 & $90 \mathrm{C}$ SCW VAPOR PHASE \\
\hline JCI 100 & $\begin{array}{l}\text { A516 } \\
\text { Grade } 55\end{array}$ & 8736 & 51.724 & 0.2693 & 6.64 & $90 \mathrm{C}$ SCW AQUEOUS PHASE \\
\hline JCl 101 & $\begin{array}{l}\text { A516 } \\
\text { Grade } 55\end{array}$ & 8736 & 52.913 & 0.2492 & 6.01 & $90 \mathrm{C}$ SCW AQUEOUS PHASE \\
\hline JCl 102 & $\begin{array}{l}\text { A516 } \\
\text { Grade } 55\end{array}$ & 8736 & 52.735 & 0.2585 & 6.25 & $90 \mathrm{C}$ SCW AQUEOUS PHASE \\
\hline KCA 097 & $\begin{array}{l}\text { A27 Grade } \\
70-40\end{array}$ & 8736 & 52.087 & 2.9636 & 72.96 & $90 \mathrm{C}$ SCW VAPOR PHASE \\
\hline KCA 098 & $\begin{array}{l}\text { A27 Grade } \\
70-40\end{array}$ & 8736 & 51.841 & 3.4384 & 85.05 & $90 \mathrm{C}$ SCW VAPOR PHASE \\
\hline KCA 099 & $\begin{array}{l}\text { A27 Grade } \\
70-40\end{array}$ & 8736 & 52.098 & 3.5607 & 87.64 & $90 \mathrm{C}$ SCW VAPOR PHASE \\
\hline KCA 100 & $\begin{array}{l}\text { A27 Grade } \\
70-40\end{array}$ & 8736 & 52.263 & 0.3425 & 8.40 & $90 \mathrm{C}$ SCW AQUEOUS PHASE \\
\hline KCA 101 & $\begin{array}{l}\text { A27 Grade } \\
70-40\end{array}$ & 8736 & 51.616 & 0.3156 & 7.84 & $90 \mathrm{C}$ SCW AQUEOUS PHASE \\
\hline KCA 102 & $\begin{array}{l}\text { A27 Grade } \\
70-40\end{array}$ & 8736 & 51.917 & 0.3336 & 8.24 & $90 \mathrm{C}$ SCW AQUEOUS PHASE \\
\hline $\mathrm{KCl} 097$ & $\begin{array}{l}\text { A27 Grade } \\
70-40\end{array}$ & 8736 & 52.816 & 4.6898 & 113.86 & $90 \mathrm{C}$ SCW VAPOR PHASE \\
\hline KCl 098 & $\begin{array}{l}\text { A27 Grade } \\
70-40\end{array}$ & 8736 & 52.384 & 5.9939 & 146.72 & $90 \mathrm{C}$ SCW VAPOR PHASE \\
\hline KCl 099 & $\begin{array}{l}\text { A27 Grade } \\
70-40\end{array}$ & 8736 & 52.160 & 8.9523 & 220.08 & $90 \mathrm{C}$ SCW VAPOR PHASE \\
\hline $\mathrm{KCl} 100$ & $\begin{array}{l}\text { A27 Grade } \\
70-40\end{array}$ & 8736 & 52.196 & 0.3202 & 7.87 & $90 \mathrm{C}$ SCW AQUEOUS PHASE \\
\hline KCl 101 & $\begin{array}{l}\text { A27 Grade } \\
70-40\end{array}$ & 8736 & 52.056 & 0.3264 & 8.04 & $90 \mathrm{C}$ SCW AQUEOUS PHASE \\
\hline KCl 102 & $\begin{array}{l}\text { A27 Grade } \\
70-40\end{array}$ & 8736 & 52.305 & 0.2984 & 7.32 & $90 \mathrm{C}$ SCW AQUEOUS PHASE \\
\hline
\end{tabular}




\section{Corrosion Rate Calculations for Crevice Specimens}

For Rack Number : 19-2

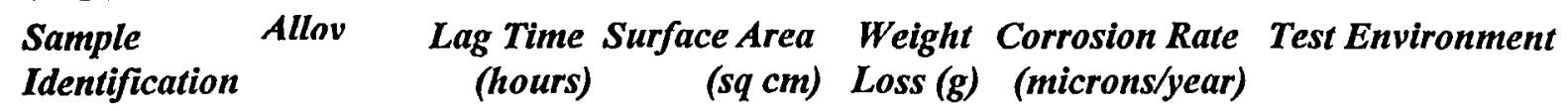

\begin{tabular}{|c|c|c|c|c|c|c|}
\hline GCA 007 & Monel 400 & 8760 & 52.279 & 0.0201 & 0.44 & $60 \mathrm{C}$ Vapor Phase SAW \\
\hline GCA 008 & Monel 400 & 8760 & 51.790 & 0.0262 & 0.57 & $60 \mathrm{C}$ Vapor Phase SAW \\
\hline GCA 009 & Monel 400 & 8760 & 52.264 & 0.0376 & 0.82 & $60 \mathrm{C}$ Vapor Phase SAW \\
\hline GCA 010 & Monel 400 & 8760 & 51.715 & 2.0265 & 44.53 & $60 \mathrm{C}$ Aqueous Phase SAW \\
\hline GCA 011 & Monel 400 & 8760 & 52.213 & 2.0635 & 44.91 & $60 \mathrm{C}$ Aqueous Phase SAW \\
\hline GCA 012 & Monel 400 & 8760 & 52.474 & 2.1587 & 46.75 & $60 \mathrm{C}$ Aqueous Phase SAW \\
\hline GCF 007 & Monel 400 & 8760 & 51.355 & 0.0502 & 1.11 & $60 \mathrm{C}$ Vapor Phase SAW \\
\hline GCF 008 & Monel 400 & 8760 & 50.811 & 0.0509 & 1.14 & $60 \mathrm{C}$ Vapor Phase SAW \\
\hline GCF 009 & Monel 400 & 8760 & 51.196 & 0.0945 & 2.10 & $60 \mathrm{C}$ Vapor Phase SAW \\
\hline GCF 010 & Monel 400 & 8760 & 51.082 & 2.9694 & 66.06 & $60 \mathrm{C}$ Aqueous Phase SAW \\
\hline GCF 011 & Monel 400 & 8760 & 51.352 & 3.0303 & 67.06 & $60 \mathrm{C}$ Aqueous Phase SAW \\
\hline GCF 012 & Monel 400 & 8760 & 51.108 & 3.2255 & 71.72 & $60 \mathrm{C}$ Aqueous Phase SAW \\
\hline HCA 007 & CDA 715 & 8760 & 53.177 & 0.0967 & 2.03 & 60 C Vapor Phase SAW \\
\hline HCA 008 & CDA 715 & 8760 & 53.288 & 0.1159 & 2.43 & $60 \mathrm{C}$ Vapor Phase SAW \\
\hline HCA 009 & CDA 715 & 8760 & 53.139 & 0.1316 & 2.77 & 60 C Vapor Phase SAW \\
\hline HCA 010 & CDA 715 & 8760 & 53.176 & 5.2422 & 110.27 & $60 \mathrm{C}$ Aqueous Phase SAW \\
\hline HCA 011 & CDA 715 & 8760 & 53.317 & 4.8345 & 101.43 & $60 \mathrm{C}$ Aqueous Phase SAW \\
\hline HCA 012 & $\operatorname{CDA} 715$ & 8760 & 53.220 & 4.9012 & 103.01 & $60 \mathrm{C}$ Aqueous Phase SAW \\
\hline HCG 007 & CDA 715 & 8760 & 51.454 & 0.1521 & 3.31 & $60 \mathrm{C}$ Vapor Phase SAW \\
\hline
\end{tabular}




\section{Corrosion Rate Calculations for Crevice Specimens}

For Rack Number : 19-2

\begin{tabular}{|c|c|c|c|c|c|c|}
\hline $\begin{array}{l}\text { Sample } \\
\text { Identification }\end{array}$ & Allov & $\begin{array}{r}\text { Lag Time } \\
\text { (hours) }\end{array}$ & $\begin{array}{r}\text { Surface Area } \\
(\mathrm{sq} \mathrm{cm})\end{array}$ & $\begin{array}{l}\text { Weight } \\
\operatorname{Loss}(g)\end{array}$ & $\begin{array}{l}\text { Corrosion Rate } \\
\text { (microns/year) }\end{array}$ & Test Environment \\
\hline HCG 008 & CDA 715 & 8760 & 51.709 & 0.1816 & 3.93 & $60 \mathrm{C}$ Vapor Phase SAW \\
\hline HCG 009 & CDA 715 & 8760 & 51.597 & 02094 & 454 & $60 \mathrm{C}$ Vapor Phase SAW \\
\hline HCG 010 & CDA 715 & 8760 & 51.349 & 4.6992 & 102.37 & $60 \mathrm{C}$ Aqueous Phase SAW \\
\hline HCG 011 & CDA 715 & 8760 & 51.586 & 4.8497 & 105.16 & $60 \mathrm{C}$ Aqueous Phase SAW \\
\hline HCG 012 & $\operatorname{CDA} 715$ & 8760 & 51.615 & 5.1464 & 111.53 & $60 \mathrm{C}$ Aqueous Phase SAW \\
\hline
\end{tabular}




\section{Corrosion Rate Calculations for Crevice Specimens}

For Rack Number : 19-1

Sample Allov Lag Time Surface Area Weight Corrosion Rate Test Environment Identification (hours) (sq cm) Loss (g) (microns/year)

\begin{tabular}{|c|c|c|c|c|c|c|}
\hline GCA 001 & Monel 400 & 4536 & 51.914 & 0.0110 & 047 & 60 C Vapor Phase SAW \\
\hline GCA 002 & Monel 400 & 4536 & 52.374 & 0.0160 & 0.67 & $60 \mathrm{C}$ Vapor Phase SAW \\
\hline GCA 003 & Monel 400 & 4536 & 51.317 & 0.0180 & 0.77 & $60 \mathrm{C}$ Vapor Phase SAW \\
\hline GCA 004 & Monel 400 & 4536 & 52.412 & 2.3345 & 97.75 & $60 \mathrm{C}$ Aqueous Phase SAW \\
\hline GCA 005 & Monel 400 & 4536 & 52.155 & 2.2476 & 94.57 & $60 \mathrm{C}$ Aqueous Phase SAW \\
\hline GCA 006 & Monel 400 & 4536 & 51.610 & 2.2643 & 96.28 & $60 \mathrm{C}$ Aqueous Phase SAW \\
\hline GCF 001 & Monel 400 & 4536 & 51.696 & 0.0162 & 0.69 & $60 \mathrm{C}$ Vapor Phase SAW \\
\hline GCF 002 & Monel 400 & 4536 & 50.647 & 0.0247 & 1.07 & $60 \mathrm{C}$ Vapor Phase SAW \\
\hline GCF 003 & Monel 400 & 4536 & 51.094 & 0.0272 & 1.17 & $60 \mathrm{C}$ Vapor Phase SAW \\
\hline GCF 004 & Monel 400 & 4536 & 51.046 & 3.0196 & 129.82 & $60 \mathrm{C}$ Aqueous Phase SAW \\
\hline GCF 005 & Monel 400 & 4536 & 50.927 & 2.9966 & 129.13 & $60 \mathrm{C}$ Aqueous Phase SAW \\
\hline GCF 006 & Monel 400 & 4536 & 51.008 & 3.5070 & 150.88 & $60 \mathrm{C}$ Aqueous Phase SAW \\
\hline HCA 001 & CDA 715 & 4536 & 53.291 & 0.0408 & 1.65 & $60 \mathrm{C}$ Vapor Phase SAW \\
\hline HCA 002 & CDA 715 & 4536 & 53.323 & 0.0480 & 1.94 & 60 C Vapor Phase SAW \\
\hline HCA 003 & CDA 715 & 4536 & 53.039 & 0.0673 & 2.74 & $60 \mathrm{C}$ Vapor Phase SAW \\
\hline HCA 004 & CDA 715 & 4536 & 53.136 & 4.2705 & 173.61 & $60 \mathrm{C}$ Aqueous Phase SAW \\
\hline HCA 005 & CDA 715 & 4536 & 52.731 & 4.4611 & 182.76 & $60 \mathrm{C}$ Aqueous Phase SAW \\
\hline HCA 006 & CDA 715 & 4536 & 52.284 & 4.4947 & 185.71 & $60 \mathrm{C}$ Aqueous Phase SAW \\
\hline HCG 001 & CDA 715 & 4536 & 51.596 & 0.0692 & 2.90 & $60 \mathrm{C}$ Vapor Phase SAW \\
\hline
\end{tabular}




\begin{tabular}{|c|c|c|c|c|c|c|}
\hline $\begin{array}{l}\text { Sample } \\
\text { Identification }\end{array}$ & Allov & $\begin{array}{r}\text { Lag Time } \\
\text { (hours) }\end{array}$ & $\begin{array}{r}\text { Surface Area } \\
(\mathrm{sq} \mathrm{cm})\end{array}$ & $\begin{array}{l}\text { Weight } \\
\operatorname{Loss}(g)\end{array}$ & $\begin{array}{l}\text { Corrosion Rate } \\
\text { (microns/year) }\end{array}$ & Test Environment \\
\hline HCG 002 & CDA 715 & 4536 & 51.491 & 0.0965 & 4.05 & 60 C Vapor Phase SAW \\
\hline HCG 003 & CDA 715 & 4536 & 51.238 & 0.1240 & 5.23 & $60 \mathrm{C}$ Vapor Phase SAW \\
\hline HCG 004 & CDA 715 & 4536 & 51.478 & 4.4934 & 188.56 & $60 \mathrm{C}$ Aqueous Phase SAW \\
\hline HCG 005 & CDA 715 & 4536 & 51.522 & 4.5945 & 192.64 & $60 \mathrm{C}$ Aqueous Phase SAW \\
\hline HCG 006 & CDA 715 & 4536 & 51.880 & 5.0990 & 212.32 & $60 \mathrm{C}$ Aqueous Phase SAW \\
\hline
\end{tabular}




\section{Corrosion Rate Calculations for Crevice Specimens}

For Rack Number : 18-2

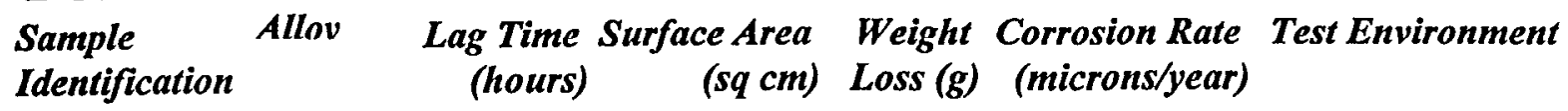

\begin{tabular}{|c|c|c|c|c|c|c|}
\hline GCA 037 & Monel 400 & 8760 & 52.286 & 0.1080 & 2.35 & $90 \mathrm{C}$ Vapor Phase SAW \\
\hline GCA 038 & Monel 400 & 8760 & 52.253 & 0.1132 & 2.46 & $90 \mathrm{C}$ Vapor Phase SAW \\
\hline GCA 039 & Monel 400 & 8760 & 52.271 & 0.1074 & 2.33 & $90 \mathrm{C}$ Vapor Phase SAW \\
\hline GCA 040 & Monel 400 & 8760 & 52.160 & 1.4891 & 32.44 & $90 \mathrm{C}$ Aqueous Phase SAW \\
\hline GCA 041 & Monel 400 & 8760 & 51.956 & 1.5235 & 33.32 & $90 \mathrm{C}$ Aqueous Phase SAW \\
\hline GCA 042 & Monel 400 & 8760 & 52.107 & 1.5811 & 34.48 & $90 \mathrm{C}$ Aqueous Phase SAW \\
\hline GCF 037 & Monel 400 & 8760 & 50.925 & 0.1333 & 2.97 & $90 \mathrm{C}$ Vapor Phase SAW \\
\hline GCF 038 & Monel 400 & 8760 & 50.887 & 0.1323 & 2.95 & $90 \mathrm{C}$ Vapor Phase SAW \\
\hline GCF 039 & Monel 400 & 8760 & 51.232 & 0.1263 & 2.80 & $90 \mathrm{C}$ Vapor Phase SAW \\
\hline GCF 040 & Monel 400 & 8760 & 50.795 & 3.1933 & 71.44 & $90 \mathrm{C}$ Aqueous Phase SAW \\
\hline GCF 041 & Monel 400 & 8760 & 51.270 & 3.4558 & 76.59 & $90 \mathrm{C}$ Aqueous Phase SAW \\
\hline GCF 042 & Monel 400 & 8760 & 51.457 & 3.7458 & 82.72 & $90 \mathrm{C}$ Aqueous Phase SAW \\
\hline HCA 037 & $\operatorname{CDA} 715$ & 8760 & 53.402 & 0.4103 & 8.59 & $90 \mathrm{C}$ Vapor Phase SAW \\
\hline HCA 038 & CDA 715 & 8760 & 53.437 & 0.3852 & 8.06 & $90 \mathrm{C}$ Vapor Phase SAW \\
\hline HCA 039 & CDA 715 & 8760 & 52.882 & 0.4099 & 8.67 & $90 \mathrm{C}$ Vapor Phase SAW \\
\hline HCA 040 & CDA 715 & 8760 & 51.838 & 7.8630 & 169.67 & $90 \mathrm{C}$ Aqueous Phase SAW \\
\hline HCA 041 & CDA 715 & 8760 & 53.067 & 11.3696 & 239.65 & $90 \mathrm{C}$ Aqueous Phase SAW \\
\hline HCA 042 & CDA 715 & 8760 & 52.742 & 10.5725 & 224.23 & $90 \mathrm{C}$ Aqueous Phase SAW \\
\hline HCG 037 & CDA 715 & 8760 & 51.837 & 0.4667 & 10.07 & $90 \mathrm{C}$ Vapor Phase SAW \\
\hline
\end{tabular}




\section{Corrosion Rate Calculations for Crevice Specimens}

For Rack Number : 18-2

\begin{tabular}{|c|c|c|c|c|c|c|}
\hline $\begin{array}{l}\text { Sample } \\
\text { Identification }\end{array}$ & Allov & $\begin{array}{c}\text { Lag Time } \\
\text { (hours) }\end{array}$ & $\begin{array}{r}\text { Surface Area } \\
(\mathrm{sq} \mathrm{cm})\end{array}$ & $\begin{array}{l}\text { Weight } \\
\text { Loss }(g)\end{array}$ & $\begin{array}{l}\text { Corrosion Rate } \\
\text { (microns/year) }\end{array}$ & Test Environment \\
\hline HCG 038 & $\operatorname{CDA} 715$ & 8760 & 51.794 & 0.3938 & 8.50 & $90 \mathrm{C}$ Vapor Phase SAW \\
\hline HCG 039 & CDA 715 & 8760 & 51559 & 0.2787 & 6.05 & 90 C Vapor Phase SAW \\
\hline HCG 040 & CDA 715 & 8760 & 51.784 & 10.4589 & 225.92 & $90 \mathrm{C}$ Aqueous Phase SAW \\
\hline HCG 041 & CDA 715 & 8760 & 51.523 & 11.1313 & 241.66 & $90 \mathrm{C}$ Aqueous Phase SAW \\
\hline HCG 042 & $\operatorname{CDA} 715$ & 8760 & 51.637 & 10.9110 & 236.36 & $90 \mathrm{C}$ Aqueous Phase SAW \\
\hline
\end{tabular}




\section{Corrosion Rate Calculations for Crevice Specimens}

For Rack Number : 18-1

Sample Allov Lag Time Surface Area Weight Corrosion Rate Test Environment Identification (hours) (sq cm) Loss (g) (microns/year)

\begin{tabular}{|c|c|c|c|c|c|c|}
\hline GCA 031 & Monel 400 & 4392 & 52.294 & 0.0583 & 2.53 & 90 C Vapor Phase SAW \\
\hline GCA 032 & Monel 400 & 4392 & 52119 & 0.0714 & 3.11 & $90 \mathrm{C}$ Vapor Phase SAW \\
\hline GCA 033 & Monel 400 & 4392 & 52.188 & 0.0689 & 2.99 & $90 \mathrm{C}$ Vapor Phase SAW \\
\hline GCA 034 & Monel 400 & 4392 & 52.214 & 1.3834 & 60.05 & $90 \mathrm{C}$ Aqueous Phase SAW \\
\hline GCA 035 & Monel 400 & 4392 & 52.145 & 1.4217 & 61.79 & $90 \mathrm{C}$ Aqueous Phase SAW \\
\hline GCA 036 & Monel 400 & 4392 & 52.440 & 1.4913 & 64.46 & $90 \mathrm{C}$ Aqueous Phase SAW \\
\hline GCF 031 & Monel 400 & 4392 & 50.447 & 0.0879 & 3.95 & $90 \mathrm{C}$ Vapor Phase SAW \\
\hline GCF 032 & Monel 400 & 4392 & 50.602 & 0.0936 & 4.19 & $90 \mathrm{C}$ Vapor Phase SAW \\
\hline GCF 033 & Monel 400 & 4392 & 50.075 & 0.0808 & 3.66 & $90 \mathrm{C}$ Vapor Phase SAW \\
\hline GCF 034 & Monel 400 & 4392 & 50.462 & 2.8835 & 129.51 & $90 \mathrm{C}$ Aqueous Phase SAW \\
\hline GCF 035 & Monel 400 & 4392 & 50.796 & 2.8827 & 128.63 & $90 \mathrm{C}$ Aqueous Phase SAW \\
\hline GCF 036 & Monel 400 & 4392 & 51.056 & 3.1031 & 137.75 & $90 \mathrm{C}$ Aqueous Phase SAW \\
\hline HCA 031 & CDA 715 & 4392 & 52.775 & 0.2102 & 8.89 & $90 \mathrm{C}$ Vapor Phase SAW \\
\hline HCA 032 & CDA 715 & 4392 & 53.159 & 0.2388 & 10.02 & $90 \mathrm{C}$ Vapor Phase SAW \\
\hline HCA 033 & CDA 715 & 4392 & 53.270 & 0.2724 & 11.41 & 90 C Vapor Phase SAW \\
\hline HCA 034 & CDA 715 & 4392 & 53.485 & 5.6175 & 234.32 & $90 \mathrm{C}$ Aqueous Phase SAW \\
\hline HCA 035 & CDA 715 & 4392 & 53.282 & 5.6138 & 235.06 & $90 \mathrm{C}$ Aqueous Phase SAW \\
\hline HCA 036 & $\operatorname{CDA} 715$ & 4392 & 53.266 & 6.0580 & 253.73 & $90 \mathrm{C}$ Aqueous Phase SAW \\
\hline HCG 031 & CDA 715 & 4392 & 51.649 & 0.2843 & 12.28 & $90 \mathrm{C}$ Vapor Phase SAW \\
\hline
\end{tabular}




\section{Corrosion Rate Calculations for Crevice Specimens}

For Rack Number : 18-1

\begin{tabular}{lcccccc}
$\begin{array}{l}\text { Sample } \\
\text { Identification }\end{array}$ & Allov & $\begin{array}{c}\text { Lag Time Surface Area } \\
\text { (hours) }\end{array}$ & $\begin{array}{c}\text { Weight } \\
\text { (sq cm) }\end{array}$ & $\begin{array}{c}\text { Corrosion }(\mathrm{g}) \\
\text { (microns/year) }\end{array}$ & \\
\hline HCG 032 & CDA 715 & 4392 & 51.408 & 0.2661 & 11.55 & 90 C Vapor Phase SAW \\
HCG 033 & CDA 715 & 4392 & 51.627 & 02507 & 10.83 & 90 C Vapor Phase SAW \\
HCG 034 & CDA 715 & 4392 & 51.060 & 7.0815 & 309.42 & 90 C Aqueous Phase SAW \\
HCG 035 & CDA 715 & 4392 & 51.935 & 7.0810 & 304.18 & 90 C Aqueous Phase SAW \\
HCG 036 & CDA 715 & 4392 & 51.826 & 7.5893 & 326.71 & 90 C Aqueous Phase SAW
\end{tabular}




\section{Corrosion Rate Calculations for Crevice Specimens} For Rack Number : 25-1
Sample Allov
Identification
Lag Time Surface Area Weight Corrosion Rate Test Environment (hours)
(sq cm) Loss (g) (microns/year)

\begin{tabular}{|c|c|c|c|c|c|c|}
\hline ACA 001 & 1825 & 4296 & 52.003 & -0.0003 & -0.01 & $60 \mathrm{C}$ Vapor Phase SAW \\
\hline ACA 002 & 1825 & 4296 & 52.189 & 0.0009 & 0.04 & $60 \mathrm{C}$ Vapor Phase SAW \\
\hline ACA 003 & 1825 & 4296 & 52.180 & 0.0015 & 0.07 & $60 \mathrm{C}$ Vapor Phase SAW \\
\hline ACA 004 & 1825 & 4296 & 52.252 & 0.0526 & 2.52 & $60 \mathrm{C}$ Aqueous Phase SAW \\
\hline ACA 005 & 1825 & 4296 & 52.317 & 0.0275 & 1.32 & $60 \mathrm{C}$ Aqueous Phase SAW \\
\hline ACA 006 & 1825 & 4296 & 52.224 & 0.0091 & 0.44 & $60 \mathrm{C}$ Aqueous Phase SAW \\
\hline ACB 001 & 1825 & 4296 & 52.104 & 0.0013 & 0.06 & $60 \mathrm{C}$ Vapor Phase SAW \\
\hline АCB 002 & 1825 & 4296 & 51.896 & 0.0019 & 0.09 & $60 \mathrm{C}$ Vapor Phase SAW \\
\hline АСВ 003 & 1825 & 4296 & 51.073 & 0.0012 & 0.06 & $60 \mathrm{C}$ Vapor Phase SAW \\
\hline ACB 004 & 1825 & 4296 & 51.879 & 0.0497 & 2.40 & $60 \mathrm{C}$ Aqueous Phase SAW \\
\hline ACB 005 & 1825 & 4296 & 51.089 & 0.0035 & 0.17 & $60 \mathrm{C}$ Aqueous Phase SAW \\
\hline ACB 006 & 1825 & 4296 & 51.696 & 0.0017 & 0.08 & $60 \mathrm{C}$ Aqueous Phase SAW \\
\hline BCA 001 & G3 & 4296 & 52.435 & 0.0017 & 0.08 & $60 \mathrm{C}$ Vapor Phase SAW \\
\hline BCA 002 & G3 & 4296 & 52.274 & 0.0016 & 0.08 & 60 C Vapor Phase SAW \\
\hline BCA 003 & G3 & 4296 & 52.507 & 0.0026 & 0.12 & $60 \mathrm{C}$ Vapor Phase SAW \\
\hline BCA 004 & G3 & 4296 & 52.440 & 0.0016 & 0.08 & $60 \mathrm{C}$ Aqueous Phase SAW \\
\hline BCA 005 & G3 & 4296 & 51.649 & 0.0014 & 0.07 & $60 \mathrm{C}$ Aqueous Phase SAW \\
\hline BCA 006 & G3 & 4296 & 52.416 & 0.0016 & 0.08 & $60 \mathrm{C}$ Aqueous Phase SAW \\
\hline BCC 001 & G3 & 4296 & 52.272 & 0.0013 & 0.06 & 60 C Vapor Phase SAW \\
\hline
\end{tabular}




\section{Corrosion Rate Calculations for Crevice Specimens}

For Rack Number : 25-1

Sample Allov Lag Time Surface Area Weight Corrosion Rate Test Environment
Identification

\begin{tabular}{|c|c|c|c|c|c|c|}
\hline $\mathrm{BCC} 002$ & G3 & 4296 & 52.256 & 0.0012 & 0.06 & $60 \mathrm{C}$ Vapor Phase SAW \\
\hline $\mathrm{BCC} 003$ & G3 & 4296 & 52.330 & 0.0010 & 0.05 & $60 \mathrm{C}$ Vapor Phase SAW \\
\hline $\mathrm{BCC} 004$ & G3 & 4296 & 51.935 & 0.0020 & 0.09 & $60 \mathrm{C}$ Aqueous Phase SAW \\
\hline BCC 005 & G3 & 4296 & 52.612 & 0.0014 & 0.07 & $60 \mathrm{C}$ Aqueous Phase SAW \\
\hline BCC 006 & G3 & 4296 & 52.434 & 0.0009 & 0.04 & $60 \mathrm{C}$ Aqueous Phase SAW \\
\hline CCA 001 & $\mathrm{C} 4$ & 4296 & 52.022 & 0.0035 & 0.16 & $60 \mathrm{C}$ Vapor Phase SAW \\
\hline CCA 002 & $\mathrm{C4}$ & 4296 & 51.911 & 0.0015 & 0.07 & $60 \mathrm{C}$ Vapor Phase SAW \\
\hline CCA 003 & $\mathrm{C} 4$ & 4296 & 52.034 & 0.0024 & 0.11 & $60 \mathrm{C}$ Vapor Phase SAW \\
\hline CCA 004 & C4 & 4296 & 51.993 & 0.0028 & 0.13 & $60 \mathrm{C}$ Aqueous Phase SAW \\
\hline CCA 005 & $\mathrm{C} 4$ & 4296 & 51.951 & 0.0022 & 0.10 & $60 \mathrm{C}$ Aqueous Phase SAW \\
\hline CCA 006 & $\mathrm{C4}$ & 4296 & 52.071 & 0.0024 & 0.11 & $60 \mathrm{C}$ Aqueous Phase SAW \\
\hline CCB 001 & $\mathrm{C} 4$ & 4296 & 51.266 & 0.0025 & 0.12 & $60 \mathrm{C}$ Vapor Phase SAW \\
\hline $\mathrm{CCB} 002$ & $\mathrm{C4}$ & 4296 & 51.257 & 0.0020 & 0.09 & $60 \mathrm{C}$ Vapor Phase SAW \\
\hline CCB 003 & $\mathrm{C4}$ & 4296 & 50.696 & 0.0024 & 0.11 & $60 \mathrm{C}$ Vapor Phase SAW \\
\hline CCB 004 & C4 & 4296 & 51.039 & 0.0043 & 0.20 & $60 \mathrm{C}$ Aqueous Phase SAW \\
\hline ССB 005 & $\mathrm{C} 4$ & 4296 & 50.664 & 0.0030 & 0.14 & $60 \mathrm{C}$ Aqueous Phase SAW \\
\hline CCB 006 & $\mathrm{C4}$ & 4296 & 51.001 & 0.0027 & 0.13 & $60 \mathrm{C}$ Aqueous Phase SAW \\
\hline DCA 001 & $\mathrm{C} 22$ & 4296 & 52.518 & 0.0019 & 0.09 & $60 \mathrm{C}$ Vapor Phase SAW \\
\hline DCA 002 & C22 & 4296 & 52.642 & 0.0025 & 0.11 & 60 C Vapor Phase SAW \\
\hline
\end{tabular}




\section{Corrosion Rate Calculations for Crevice Specimens} For Rack Number : 25-1
Sample Allov
Identification
Lag Time Surface Area Weight Corrosion Rate Test Environment (hours) (sq cm) Loss (g) (microns/year)

\begin{tabular}{|c|c|c|c|c|c|c|}
\hline DCA 003 & $\mathrm{C} 22$ & 4296 & 52.355 & 0.0017 & 0.08 & $60 \mathrm{C}$ Vapor Phase SAW \\
\hline DCA 004 & $\mathrm{C} 22$ & 4296 & 52.570 & 0.0023 & 0.10 & $60 \mathrm{C}$ Aqueous Phase SAW \\
\hline DCA 005 & $\mathrm{C} 22$ & 4296 & 52.642 & 0.0018 & 0.08 & $60 \mathrm{C}$ Aqueous Phase SAW \\
\hline DCA 006 & $\mathrm{C} 22$ & 4296 & 52.883 & 0.0026 & 0.12 & $60 \mathrm{C}$ Aqueous Phase SAW \\
\hline DCB 001 & $\mathrm{C} 22$ & 4296 & 50.710 & 0.0006 & 0.03 & $60 \mathrm{C}$ Vapor Phase SAW \\
\hline DCB 002 & $\mathrm{C} 22$ & 4296 & 50.632 & 0.0004 & 0.02 & $60 \mathrm{C}$ Vapor Phase SAW \\
\hline DCB 003 & $\mathrm{C} 22$ & 4296 & 50.950 & 0.0002 & 0.01 & 60 C Vapor Phase SAW \\
\hline DCB 004 & $\mathrm{C} 22$ & 4296 & 50.624 & 0.0014 & 0.07 & $60 \mathrm{C}$ Aqueous Phase SAW \\
\hline DCB 005 & $\mathrm{C} 22$ & 4296 & 50.205 & 0.0014 & 0.07 & $60 \mathrm{C}$ Aqueous Phase SAW \\
\hline DCB 006 & $\mathrm{C} 22$ & 4296 & 50.378 & 0.0010 & 0.05 & $60 \mathrm{C}$ Aqueous Phase SAW \\
\hline ECA 001 & Ti Grade 12 & 4296 & 52.244 & 0.0567 & 5.00 & $60 \mathrm{C}$ Vapor Phase SAW \\
\hline ECA 002 & Ti Grade 12 & 4296 & 53.564 & 0.0555 & 4.77 & 60 C Vapor Phase SAW \\
\hline ECA 003 & Ti Grade 12 & 4296 & 50.303 & 0.0936 & 8.56 & $60 \mathrm{C}$ Vapor Phase SAW \\
\hline ECA 004 & Ti Grade 12 & 4296 & 51.921 & 0.0855 & 7.58 & $60 \mathrm{C}$ Aqueous Phase SAW \\
\hline ECA 005 & TI Grade 12 & 4296 & 52.941 & 0.0649 & 5.64 & $60 \mathrm{C}$ Aqueous Phase SAW \\
\hline ECA 006 & Ti Grade 12 & 4296 & 49.193 & 0.0812 & 7.60 & $60 \mathrm{C}$ Aqueous Phase SAW \\
\hline ECD 001 & Ti Grade 12 & 4296 & $\mathbf{5 3 . 5 8 7}$ & 0.0567 & 4.87 & 60 C Vapor Phase SAW \\
\hline ECD 002 & Ti Grade 12 & 4296 & 53.692 & 0.0445 & 3.81 & 60 C Vapor Phase SAW \\
\hline ECD 003 & Ti Grade 12 & 4296 & 53.931 & 0.0880 & 7.51 & 60 C Vapor Phase SAW \\
\hline
\end{tabular}




\section{Corrosion Rate Calculations for Crevice Specimens}

\section{For Rack Number : 25-1}

$\begin{array}{lcrlll}\text { Sample Allov Lag Time Surface Area } & \text { Weight Corrosion Rate } & \text { Test Environment } \\ \text { Identification } & \text { (hours) } & (\mathrm{sq} \mathrm{cm}) & \text { Loss }(\mathrm{g}) & \text { (microns/year) }\end{array}$

\begin{tabular}{|c|c|c|c|c|c|c|}
\hline ECD 004 & Ti Grade 12 & 4296 & 63.737 & 0.0278 & 2.38 & $60 \mathrm{C}$ Aqueous Phase SAW \\
\hline ECD 005 & Ti Grade 12 & 4296 & 53.570 & 0.0297 & 2.55 & $60 \mathrm{C}$ Aqueous Phase SAW \\
\hline ECD 006 & Ti Grade 12 & 4296 & 53.758 & 0.0312 & 2.67 & $60 \mathrm{C}$ Aqueous Phase SAW \\
\hline FCA 001 & Ti Grade 16 & 4296 & 52.530 & 0.1175 & 10.09 & $60 \mathrm{C}$ Vapor Phase SAW \\
\hline FCA 002 & Ti Grade 16 & 4296 & 52.016 & 0.0450 & 3.90 & $60 \mathrm{C}$ Vapor Phase SAW \\
\hline FCA 003 & Ti Grade 16 & 4296 & 52.471 & 0.0449 & 3.86 & $60 \mathrm{C}$ Vapor Phase SAW \\
\hline FCA 004 & Ti Grade 16 & 4296 & 52.776 & 0.0415 & 3.55 & $60 \mathrm{C}$ Aqueous Phase SAW \\
\hline FCA 005 & Ti Grade 16 & 4296 & 52.541 & 0.0563 & 4.83 & $60 \mathrm{C}$ Aqueous Phase SAW \\
\hline FCA 006 & Ti Grade 16 & 4296 & 52.387 & 0.0417 & 3.59 & $60 \mathrm{C}$ Aqueous Phase SAW \\
\hline FCE 001 & Ti Grade 16 & 4296 & 51.856 & 0.0679 & 5.91 & $60 \mathrm{C}$ Vapor Phase SAW \\
\hline FCE 002 & Ti Grade 16 & 4296 & 51.572 & 0.0897 & 7.85 & $60 \mathrm{C}$ Vapor Phase SAW \\
\hline FCE 003 & Ti Grade 16 & 4296 & 51.842 & 0.0588 & 5.12 & 60 C Vapor Phase SAW \\
\hline FCE 004 & Ti Grade 16 & 4296 & 51.341 & 0.0397 & 3.49 & $60 \mathrm{C}$ Aqueous Phase SAW \\
\hline FCE 005 & Ti Grade 16 & 4296 & 51.828 & 0.0182 & 1.58 & $60 \mathrm{C}$ Aqueous Phase SAW \\
\hline FCE 006 & Ti Grade 16 & 4296 & 51.837 & 0.0249 & 2.17 & $60 \mathrm{C}$ Aqueous Phase SAW \\
\hline LCA 001 & Inconel 625 & 4296 & 52.180 & -0.0001 & 0.00 & $60 \mathrm{C}$ Vapor Phase SAW \\
\hline LCA 002 & Inconel 625 & 4296 & 51.520 & 0.0001 & 0.00 & $60 \mathrm{C}$ Vapor Phase SAW \\
\hline LCA 003 & Inconel 625 & 4296 & 52.374 & 0.0183 & $0: 64$ & $60 \mathrm{C}$ Vapor Phase SAW \\
\hline LCA 004 & Inconel 625 & 4296 & 51.664 & 0.0018 & 0.08 & $60 \mathrm{C}$ Aqueous Phase SAW \\
\hline
\end{tabular}




\section{Corrosion Rate Calculations for Crevice Specimens For Rack Number : 25-1 \\ $\begin{array}{lcc}\text { Sample Allov } & \text { Lag Time Surface Area Weight Corrosion Rate Test Environment } \\ \text { Identification } & \text { (hours) } & \text { (sq cm) Loss (g) (microns/year) }\end{array}$}

\begin{tabular}{|c|c|c|c|c|c|c|}
\hline LCA 005 & Inconel 625 & 4296 & 52.042 & 0.0007 & 0.03 & $60 \mathrm{C}$ Aqueous Phase SAW \\
\hline LCA 006 & Inconel 625 & 4296 & 52.130 & 0.0007 & 0.03 & $60 \mathrm{C}$ Aqueous Phase SAW \\
\hline LCJ 001 & Inconel 625 & 4296 & 51.208 & -0.0004 & -0.02 & $60 \mathrm{C}$ Vapor Phase SAW \\
\hline LCJ 002 & Inconel 625 & 4296 & 50.521 & -0.0003 & 0.01 & $60 \mathrm{C}$ Vapor Phase SAW \\
\hline LCJ 003 & Inconel 625 & 4296 & 51.452 & -0.0003 & 0.01 & $60 \mathrm{C}$ Vapor Phase SAW \\
\hline LCJ 004 & Inconel 625 & 4296 & 51.587 & 0.0007 & 0.03 & $60 \mathrm{C}$ Aqueous Phase SAW \\
\hline LCJ 005 & Inconel 625 & 4296 & 51.440 & 0.0011 & 0.05 & $60 \mathrm{C}$ Aqueous Phase SAW \\
\hline LCa 006 & Inconel 625 & 4296 & 51.117 & 0.0016 & 0.08 & $60 \mathrm{C}$ Aqueous Phase SAW \\
\hline
\end{tabular}




\section{Corrosion Rate Calculations for Crevice Specimens}

For Rack Number : 25-2

Sample Allov Lag Time Surface Area Weight Corrosion Rate Test Environment Identification

(hours) (sq cm) Loss (g) (microns/year)

\begin{tabular}{|c|c|c|c|c|c|c|}
\hline DCA 007 & $\mathrm{C} 22$ & 8376 & 51.988 & 0.0018 & 0.04 & $60 \mathrm{C}$ Vapor Phase SAW \\
\hline DCA 008 & $\mathrm{C} 22$ & 8376 & 52.691 & 0.0022 & 0.05 & $60 \mathrm{C}$ Vapor Phase SAW \\
\hline DCA 009 & $\mathrm{C} 22$ & 8376 & 52.398 & 0.0038 & 0,09 & $60 \mathrm{C}$ Vapor Phase SAW \\
\hline DCA 010 & $\mathrm{C} 22$ & 8376 & 52.868 & 0.0022 & 0.05 & $60 \mathrm{C}$ Aqueous Phase SAW \\
\hline DCA 011 & $\mathrm{C} 22$ & 8376 & 52.761 & 0.0018 & 0.04 & $60 \mathrm{C}$ Aqueous Phase SAW \\
\hline DCA 012 & $\mathrm{C} 22$ & 8376 & 52.500 & 0.0021 & 0.05 & $60 \mathrm{C}$ Aqueous Phase SAW \\
\hline DCB 007 & $\mathrm{C} 22$ & 8376 & 50.795 & 0.0010 & 602 & $60 \mathrm{C}$ Vapor Phase SAW \\
\hline DCB 008 & $\mathrm{C} 22$ & 8376 & 50.083 & 0.0011 & 0.03 & $60 \mathrm{C}$ Vapor Phase SAW \\
\hline DCB 009 & $\mathrm{C} 22$ & 8376 & 50.252 & 0.0012 & 0,03 & $60 \mathrm{C}$ Vapor Phase SAW \\
\hline DCB 010 & $\mathrm{C} 22$ & 8376 & 51.334 & 0.0041 & 0.10 & $60 \mathrm{C}$ Aqueous Phase SAW \\
\hline DCB 011 & $\mathrm{C} 22$ & 8376 & 50.647 & 0.0015 & 0.04 & $60 \mathrm{C}$ Aqueous Phase SAW \\
\hline DCB 012 & $\mathrm{C} 22$ & 8376 & 50.329 & 0.0019 & 0.05 & $60 \mathrm{C}$ Aqueous Phase SAW \\
\hline LCA 007 & Inconel 625 & 8376 & 51.589 & 0.0011 & 0.03 & $60 \mathrm{C}$ Vapor Phase SAW \\
\hline LCA 008 & inconel 625 & 8376 & 51.438 & 0.0002 & 0.00 & 60 C Vapor Phase SAW \\
\hline LCA 009 & Inconel 625 & 8376 & 52.398 & 0.0007 & $6: 02$ & 60 C Vapor Phase SAW \\
\hline LCA 010 & Inconel 625 & 8376 & 50.907 & 0.0014 & 0.03 & $60 \mathrm{C}$ Aqueous Phase SAW \\
\hline LCA 011 & Inconel 625 & 8376 & 52.279 & 0.0012 & 0.03 & $60 \mathrm{C}$ Aqueous Phase SAW \\
\hline LCA 012 & Inconel 625 & 8376 & 52.525 & 0.0008 & 0.02 & $60 \mathrm{C}$ Aqueous Phase SAW \\
\hline LCJ 007 & Inconel 625 & 8376 & 51.421 & 0.0004 & 0.01 & $60 \mathrm{C}$ Vapor Phase SAW \\
\hline
\end{tabular}




\section{Corrosion Rate Calculations for Crevice Specimens}

\section{For Rack Number : 25-2}

$\begin{aligned} & \text { Sample Allov Lag Time Surface Area Weight Corrosion Rate Test Environment } \\ & \text { Identification }\end{aligned}$
(hours) $(\mathrm{sq} \mathrm{cm})$ Loss $(\mathrm{g})$ (microns/year)

\begin{tabular}{llccccc}
\hline LCJ 008 & Inconel 625 & 8376 & 51.614 & 0.0007 & 0.02 & 60 C Vapor Phase SAW \\
LCJ 009 & Inconel 625 & 8376 & 51.361 & 0.0009 & 0.02 & 60 C Vapor Phase SAW \\
LCJ 010 & Inconel 625 & 8376 & 51.166 & 00015 & 0.04 & 60 C Aqueous Phase SAW \\
LCJ 011 & Inconel 625 & 8376 & 51.343 & 0.0024 & 0.06 & 60 C Aqueous Phase SAW \\
LCJ 012 & Inconel 625 & 8376 & 51.266 & 0.0019 & 0.05 & 60 C Aqueous Phase SAW
\end{tabular}




\section{Corrosion Rate Calculations for Crevice Specimens}

For Rack Number : 26-1

Sample Allov Lag Time Surface Area Weight Corrosion Rate Test Environment Identification (hours) (sq cm) Loss (g) (microns/year)

\begin{tabular}{|c|c|c|c|c|c|c|}
\hline ACA 031 & 1825 & 4344 & 52447 & 0.0029 & 0.14 & $90 \mathrm{C}$ Vapor Phase SAW \\
\hline ACA 032 & 1825 & 4344 & 51.808 & 0.0017 & $0: 08$ & $90 \mathrm{C}$ Vapor Phase SAW \\
\hline ACA 033 & 1825 & 4344 & 52.521 & 0.0015 & 007 & $90 \mathrm{C}$ Vapor Phase SAW \\
\hline ACA 034 & 1825 & 4344 & 52.796 & 0.0634 & 2.87 & $90 \mathrm{C}$ Aqueous Phase SAW \\
\hline ACA 035 & 1825 & 4344 & 51.897 & 0.0571 & 2.73 & $90 \mathrm{C}$ Aqueous Phase SAW \\
\hline ACA 036 & 1825 & 4344 & 51.832 & 0.0670 & 3.20 & $90 \mathrm{C}$ Aqueous Phase SAW \\
\hline ACB 031 & 1825 & 4344 & 51.846 & 0.0010 & 005 & $90 \mathrm{C}$ Vapor Phase SAW \\
\hline ACB 032 & 1825 & 4344 & 52.083 & 0.0019 & 0.09 & $90 \mathrm{C}$ Vapor Phase SAW \\
\hline ACB 033 & 1825 & 4344 & 51.292 & 0.0015 & $0: 07$ & $90 \mathrm{C}$ Vapor Phase SAW \\
\hline ACB 034 & 1825 & 4344 & 51.148 & 0.0427 & 2.07 & $90 \mathrm{C}$ Aqueous Phase SAW \\
\hline ACB 035 & 1825 & 4344 & 51.895 & 0.0281 & 1.34 & $90 \mathrm{C}$ Aqueous Phase SAW \\
\hline ACB 036 & 1825 & 4344 & 52.670 & 0.0476 & 2.24 & $90 \mathrm{C}$ Aqueous Phase SAW \\
\hline BCA 031 & G3 & 4344 & 52.701 & 0.0020 & 0.09 & $90 \mathrm{C}$ Vapor Phase SAW \\
\hline BCA 032 & G3 & 4344 & 51.762 & 0.0008 & 0.04 & $90 \mathrm{C}$ Vapor Phase SAW \\
\hline BCA 033 & G3 & 4344 & 52.617 & 0.0018 & 0.08 & $90 \mathrm{C}$ Vapor Phase SAW \\
\hline BCA 034 & G3 & 4344 & 51.678 & 0.0031 & 0.15 & $90 \mathrm{C}$ Aqueous Phase SAW \\
\hline BCA 035 & G3 & 4344 & 51.868 & 0.0022 & 0.10 & 90 C Aqueous Phase SAW \\
\hline BCA 036 & G3 & 4344 & 52.549 & 0.0016 & 0.07 & $90 \mathrm{C}$ Aqueous Phase SAW \\
\hline BCC 031 & G3 & 4344 & 52.538 & 0.0006 & 0.03 & $90 \mathrm{C}$ Vapor Phase SAW \\
\hline
\end{tabular}




\section{Corrosion Rate Calculations for Crevice Specimens For Rack Number : 26-1}

$\begin{array}{llrlll}\text { Sample Allov Lag Time Surface Area } & \text { Weight Corrosion Rate } & \text { Test Environment } \\ \text { Identification } & \text { (hours) } & \text { (sq cm) } & \text { Loss (g) } & \text { (microns/year) }\end{array}$

\begin{tabular}{|c|c|c|c|c|c|c|}
\hline BCC 032 & G3 & 4344 & 52.740 & 0.0012 & 0.06 & 90 C Vapor Phase SAW \\
\hline BCC 033 & G3 & 4344 & 52.363 & 0.0013 & 0.06 & 90 C Vapor Phase SAW \\
\hline BCC 034 & G3 & 4344 & 52.845 & 0.0013 & 0.06 & $90 \mathrm{C}$ Aqueous Phase SAW \\
\hline BCC 035 & G3 & 4344 & 52.377 & 0.0011 & 0.05 & $90 \mathrm{C}$ Aqueous Phase SAW \\
\hline BCC 036 & G3 & 4344 & 52.608 & 0.0223 & 1.03 & $90 \mathrm{C}$ Aqueous Phase SAW \\
\hline CCA 031 & C4 & 4344 & 52.302 & 0.0009 & 0.04 & 90 C Vapor Phase SAW \\
\hline CCA 032 & $\mathrm{C}_{4}$ & 4344 & 50.779 & 0.0008 & 0.04 & 90 C Vapor Phase SAW \\
\hline CCA 033 & $\mathrm{C} 4$ & 4344 & 51.456 & 0.0005 & 0.02 & $90 \mathrm{C}$ Vapor Phase SAW \\
\hline CCA 034 & C4 & 4344 & 51.392 & 0.0058 & 0.26 & $90 \mathrm{C}$ Aqueous Phase SAW \\
\hline CCA 035 & $\mathrm{C} 4$ & 4344 & 51.429 & 0.0048 & 0.22 & $90 \mathrm{C}$ Aqueous Phase SAW \\
\hline CCA 036 & $\mathrm{C} 4$ & 4344 & 51.332 & 0.0057 & 0.26 & $90 \mathrm{C}$ Aqueous Phase SAW \\
\hline CCB 031 & $\mathrm{C}_{4}$ & 4344 & 51.948 & 0.0042 & 0.19 & $90 \mathrm{C}$ Vapor Phase SAW \\
\hline ССВ 032 & $\mathrm{C} 4$ & 4344 & 51.189 & 0.0018 & 0.08 & $90 \mathrm{C}$ Vapor Phase SAW \\
\hline CCB 033 & $\mathrm{C}_{4}$ & 4344 & 50.548 & 0.0012 & 0.06 & $90 \mathrm{C}$ Vapor Phase SAW \\
\hline CCB 034 & C4 & 4344 & 51.296 & 0.0036 & 0.16 & $90 \mathrm{C}$ Aqueous Phase SAW \\
\hline ССв 035 & $\mathrm{C}_{4}$ & 4344 & 50.859 & 0.0035 & 0.16 & $80 \mathrm{C}$ Aqueous Phase SAW \\
\hline ССВ 036 & C4 & 4344 & 52.051 & 0.0047 & 0.21 & $90 \mathrm{C}$ Aqueous Phase SAW \\
\hline DCA 031 & C22 & 4344 & 52.637 & 0.0017 & 0.08 & 90 C Vapor Phase SAW \\
\hline DCA 032 & $\mathrm{C} 22$ & 4344 & 51.561 & 0.0012 & 0.05 & $90 \mathrm{C}$ Vapor Phase SAW \\
\hline
\end{tabular}




\title{
Corrosion Rate Calculations for Crevice Specimens
} For Rack Number : 26-1

\author{
Sample Allov Lag Time Surface Area Weight Corrosion Rate Test Environment \\ Identification (hours) (sq cm) Loss (g) (microns/year)
}

\begin{tabular}{|c|c|c|c|c|c|c|}
\hline DCA 033 & $\mathrm{C} 22$ & 4344 & 52.828 & 0.0022 & 0.10 & $90 \mathrm{C}$ Vapor Phase SAW \\
\hline DCA 034 & $\mathrm{C} 22$ & 4344 & 52.653 & 0.0055 & 0.24 & $90 \mathrm{C}$ Aqueous Phase SAW \\
\hline DCA 035 & $\mathrm{C} 22$ & 4344 & 52.624 & 0.0164 & 0.73 & $90 \mathrm{C}$ Aqueous Phase SAW \\
\hline DCA 036 & C22 & 4344 & 52.026 & 0.0039 & 0.18 & $90 \mathrm{C}$ Aqueous Phase SAW \\
\hline DCB 031 & C22 & 4344 & 50.396 & 0.0011 & 0.05 & $90 \mathrm{C}$ Vapor Phase SAW \\
\hline DCB 032 & $\mathrm{C} 22$ & 4344 & 50.210 & 0.0010 & 0.05 & $90 \mathrm{C}$ Vapor Phase SAW \\
\hline DCB 033 & $\mathrm{C} 22$ & 4344 & 50.454 & 0.0015 & 0.07 & 90 C Vapor Phase SAW \\
\hline DCB 034 & $\mathrm{C} 22$ & 4344 & 50.252 & 0.0000 & 0.00 & $90 \mathrm{C}$ Aqueous Phase SAW \\
\hline DCB 035 & $\mathrm{C} 22$ & 4344 & 50.700 & 0.0009 & 0.04 & $90 \mathrm{C}$ Aqueous Phase SAW \\
\hline DCB 036 & $\mathrm{C} 22$ & 4344 & 50.294 & 0.0023 & 0.11 & $90 \mathrm{C}$ Aqueous Phase SAW \\
\hline ECA 031 & Ti Grade 12 & 4344 & 53.014 & 0.1173 & 10.07 & $90 \mathrm{C}$ Vapor Phase SAW \\
\hline ECA 032 & Ti Grade 12 & 4344 & 53.150 & 0.0934 & 8.00 & $90 \mathrm{C}$ Vapor Phase SAW \\
\hline ECA 033 & Ti Grade 12 & 4344 & 53.688 & 0.0477 & 4.04 & $90 \mathrm{C}$ Vapor Phase SAW \\
\hline ECA 034 & $\pi$ Grade 12 & 4344 & 53.741 & 0.0063 & 0.53 & $90 \mathrm{C}$ Aqueous Phase SAW \\
\hline ECA 035 & TI Grade 12 & 4344 & 50.804 & 0.0501 & 4.49 & $90 \mathrm{C}$ Aqueous Phase SAW \\
\hline ECA 036 & Ti Grade 12 & 4344 & $\mathbf{5 0 . 3 8 0}$ & 0.0583 & 5.27 & $90 \mathrm{C}$ Aqueous Phase SAW \\
\hline ECD 031 & Ti Grade 12 & 4344 & 53.622 & 0.1265 & 10.74 & 90 C Vapor Phase SAW \\
\hline ECD 032 & Ti Grade 12 & 4344 & $\mathbf{5 3 . 4 4 9}$ & 0.0797 & 6.79 & $90 \mathrm{C}$ Vapor Phase SAW \\
\hline ECD 033 & TI Grade 12 & 4344 & 53.930 & 0.1362 & 11.50 & 90 C Vapor Phase SAW \\
\hline
\end{tabular}




\title{
Corrosion Rate Calculations for Crevice Specimens
}

\section{For Rack Number : 26-1}

\author{
Sample Allov Lag Time Surface Area Weight Corrosion Rate Test Environment \\ Identification (hours) (sq cm) Loss (g) (microns/year)
}

\begin{tabular}{|c|c|c|c|c|c|c|}
\hline ECD 034 & Ti Grade 12 & 4344 & 53.961 & 0.0804 & 6.78 & $90 \mathrm{C}$ Aqueous Phase SAW \\
\hline ECD 035 & Ti Grade 12 & 4344 & 53.608 & 0.0468 & 3.97 & $90 \mathrm{C}$ Aqueous Phase SAW \\
\hline ECD 036 & Ti Grade 12 & 4344 & 53.917 & 0.0478 & 4.04 & $90 \mathrm{C}$ Aqueous Phase SAW \\
\hline FCA 031 & Ti Grade 16 & 4344 & 52.253 & 0.0541 & 4.62 & $90 \mathrm{C}$ Vapor Phase SAW \\
\hline FCA 032 & Ti Grade 16 & 4344 & 52.425 & 0.0575 & 4.89 & 90 C Vapor Phase SAW \\
\hline FCA 033 & Ti Grade 16 & 4344 & 52.127 & 0.0793 & 6.79 & 90 C Vapor Phase SAW \\
\hline FCA 034 & Ti Grade 16 & 4344 & 51.984 & 0.0216 & 1.85 & $90 \mathrm{C}$ Aqueous Phase SAW \\
\hline FCA 035 & Ti Grade 16 & 4344 & 52.376 & 0.0186 & 1.58 & $90 \mathrm{C}$ Aqueous Phase SAW \\
\hline FCA 036 & Ti Grade 16 & 4344 & 52.294 & 0.0322 & 2.75 & $90 \mathrm{C}$ Aqueous Phase SAW \\
\hline FCE 031 & Ti Grade 16 & 4344 & 51.893 & 0.0580 & 4.99 & $90 \mathrm{C}$ Vapor Phase SAW \\
\hline FCE 032 & Ti Grade 16 & 4344 & 51.741 & 0.0649 & 5.60 & $90 \mathrm{C}$ Vapor Phase SAW \\
\hline FCE 033 & Ti Grade 16 & 4344 & 50.798 & 0.0508 & 4.46 & $90 \mathrm{C}$ Vapor Phase SAW \\
\hline FCE 034 & Ti Grade 16 & 4344 & 50.923 & 0.0322 & 2.82 & $90 \mathrm{C}$ Aqueous Phase SAW \\
\hline FCE 035 & Ti Grade 16 & 4344 & 51.809 & 0.0409 & 3.52 & $90 \mathrm{C}$ Aqueous Phase SAW \\
\hline FCE 036 & Ti Grade 16 & 4344 & 51.172 & 0.0289 & 2.52 & $90 \mathrm{C}$ Aqueous Phase SAW \\
\hline LCA 031 & Inconel 625 & 4344 & 52.868 & -0.0001 & 0,00 & 90 C Vapor Phase SAW \\
\hline LCA 032 & Inconel 625 & 4344 & 52.171 & 0.0013 & 0.06 & 90 C Vapor Phase SAW \\
\hline LCA 033 & Inconel 625 & 4344 & 52.211 & -0.0001 & 0.00 & 90 C Vapor Phase SAW \\
\hline LCA 034 & Inconel 625 & 4344 & 52.436 & 0.0002 & 0.01 & $90 \mathrm{C}$ Aqueous Phase SAW \\
\hline
\end{tabular}




\section{Corrosion Rate Calculations for Crevice Specimens} For Rack Number : 26-1
Sample Allov
Identification
Lag Time Surface Area
Weight Corrosion Rate Test Environment

\section{Identification}

\begin{tabular}{|c|c|c|c|c|c|c|}
\hline LCA 035 & Inconel 625 & 4344 & 52.485 & 0.0005 & 0.02 & $90 \mathrm{C}$ Aqueous Phase SAW \\
\hline LCA 036 & Inconel 625 & 4344 & 52360 & 0.0000 & 0.00 & $90 \mathrm{C}$ Aqueous Phase SAW \\
\hline LCJ 031 & Inconel 625 & 4344 & 51.568 & 0.0001 & 0,00 & $90 \mathrm{C}$ Vapor Phase SAW \\
\hline LCJ 032 & Inconel 625 & 4344 & 51.531 & 0.0007 & 0.03 & 90 C Vapor Phase SAW \\
\hline LCJ 033 & Inconel 625 & 4344 & 51.668 & 0.0004 & 602 & $90 \mathrm{C}$ Vapor Phase SAW \\
\hline LCJ 034 & Inconel 625 & 4344 & 51.473 & 0.0007 & 0.03 & $90 \mathrm{C}$ Aqueous Phase SAW \\
\hline LCJ 035 & Inconel 625 & 4344 & 51.418 & 0.0000 & 0.00 & $90 \mathrm{C}$ Aqueous Phase SAW \\
\hline LCJ 036 & Inconel 625 & 4344 & 50.410 & -0.0006 & -0.03 & $90 \mathrm{C}$ Aqueous Phase SAW \\
\hline
\end{tabular}




\section{Corrosion Rate Calculations for Crevice Specimens} For Rack Number : 27-1
Sample
Allov
Identification
Lag Time Surface Area
Weight Corrosion Rate Test Environment (hours) (sq cm) Loss (g) (microns/year)

\begin{tabular}{|c|c|c|c|c|c|c|}
\hline ACA 061 & 1825 & 4392 & 52755 & 0.0013 & 0.06 & $60 \mathrm{C}$ Vapor Phase SCW \\
\hline ACA 062 & 1825 & 4392 & 51.584 & 0.0014 & 0.07 & 60 C Vapor Phase SCW \\
\hline ACA 063 & 1825 & 4392 & 52.651 & 0.0015 & 0.07 & $60 \mathrm{C}$ Vapor Phase SCW \\
\hline ACA 064 & 1825 & 4392 & 51.905 & 0.0012 & 0.06 & $60 \mathrm{C}$ Aqueous Phase SCW \\
\hline ACA 065 & 1825 & 4392 & 52.662 & 0.0023 & 0.11 & $60 \mathrm{C}$ Aqueous Phase SCW \\
\hline ACA 066 & 1825 & 4392 & 52.406 & 0.0018 & 0.08 & $60 \mathrm{C}$ Aqueous Phase SCW \\
\hline АСB 061 & 1825 & 4392 & 52.251 & 0.0010 & 0.05 & $60 \mathrm{C}$ Vapor Phase SCW \\
\hline АСВ 062 & 1825 & 4392 & 51.994 & 0.0014 & 0.07 & $60 \mathrm{C}$ Vapor Phase SCW \\
\hline АСВ 063 & 1825 & 4392 & 51.621 & 0.0015 & 0.07 & $60 \mathrm{C}$ Vapor Phase SCW \\
\hline АСB 064 & 1825 & 4392 & 51.750 & 0.0020 & 0.09 & $60 \mathrm{C}$ Aqueous Phase SCW \\
\hline АCB 065 & 1825 & 4392 & 51.928 & 0.0033 & 0.16 & $60 \mathrm{C}$ Aqueous Phase SCW \\
\hline АСB 066 & 1825 & 4392 & 51.970 & 0.0016 & 0.08 & $60 \mathrm{C}$ Aqueous Phase SCW \\
\hline BCA 061 & G3 & 4392 & 52.228 & 0.0018 & 0.08 & $60 \mathrm{C}$ Vapor Phase SCW \\
\hline BCA 062 & G3 & 4392 & 52.635 & 0.0017 & 0.08 & 60 C Vapor Phase SCW \\
\hline BCA 063 & G3 & 4392 & 51.984 & 0.0012 & 0.06 & $60 \mathrm{C}$ Vapor Phase SCW \\
\hline BCA 064 & G3 & 4392 & 52.862 & 0.0042 & 0.19 & $60 \mathrm{C}$ Aqueous Phase SCW \\
\hline BCA 065 & G3 & 4392 & 52.669 & 0.0037 & 0.17 & $60 \mathrm{C}$ Aqueous Phase SCW \\
\hline BCA 066 & G3 & 4392 & 51.430 & 0.0039 & 0.15 & $60 \mathrm{C}$ Aqueous Phase SCW \\
\hline BCC 061 & G3 & 4392 & 52.335 & 0.0019 & 0.09 & 60 C Vapor Phase SCW \\
\hline
\end{tabular}




\section{Corrosion Rate Calculations for Crevice Specimens}

For Rack Number : 27-1

Sample Allov Lag Time Surface Area Weight Corrosion Rate Test Environment
Identification
(hours) (sq cm) Loss $(\mathrm{g})$ (microns/year)

\begin{tabular}{|c|c|c|c|c|c|c|}
\hline BCC 062 & G3 & 4392 & 52.309 & 0.0016 & 0.07 & $60 \mathrm{C}$ Vapor Phase SCW \\
\hline $\mathrm{BCC} 063$ & G3 & 4392 & 52.101 & 0.0013 & 0.06 & $60 \mathrm{C}$ Vapor Phase SCW \\
\hline BCC 064 & G3 & 4392 & 52.665 & 0.0022 & 0.10 & $60 \mathrm{C}$ Aqueous Phase SCW \\
\hline BCC 065 & G3 & 4392 & 51.883 & 0.0020 & 0.09 & $60 \mathrm{C}$ Aqueous Phase SCW \\
\hline BCC 066 & G3 & 4392 & 52.016 & 0.0026 & 0.12 & $60 \mathrm{C}$ Aqueous Phase SCW \\
\hline CCA 061 & $\mathrm{C}_{4}$ & 4392 & 51.459 & 0.0022 & 0.10 & $60 \mathrm{C}$ Vapor Phase SCW \\
\hline CCA 062 & $\mathrm{C} 4$ & 4392 & 52.084 & 0.0017 & 0.08 & $60 \mathrm{C}$ Vapor Phase SCW \\
\hline CCA 063 & C4 & 4392 & 52.166 & 0.0019 & 0.08 & $60 \mathrm{C}$ Vapor Phase SCW \\
\hline CCA 064 & $\mathrm{C} 4$ & 4392 & 51.895 & 0.0025 & 0.11 & $60 \mathrm{C}$ Aqueous Phase SCW \\
\hline CCA 065 & $\mathrm{C} 4$ & 4392 & 51.719 & 0.0024 & 0.11 & $60 \mathrm{C}$ Aqueous Phase SCW \\
\hline CCA 066 & $\mathrm{C} 4$ & 4392 & 51.676 & 0.0038 & 0.17 & $60 \mathrm{C}$ Aqueous Phase SCW \\
\hline CСB 061 & C4 & 4392 & 51.755 & 0.0008 & 0.04 & $60 \mathrm{C}$ Vapor Phase SCW \\
\hline ССВ 062 & $\mathrm{C}_{4}$ & 4392 & 51.626 & 0.0011 & 0.05 & $60 \mathrm{C}$ Vapor Phase SCW \\
\hline ССВ 063 & C4 & 4392 & 51.394 & 0.0009 & 0.04 & $60 \mathrm{C}$ Vapor Phase SCW \\
\hline CCB 064 & C4 & 4392 & 51.471 & 0.0026 & 0.12 & $60 \mathrm{C}$ Aqueous Phase $\mathrm{SCW}$ \\
\hline CСB 065 & $\mathrm{C4}$ & 4392 & 51.139 & 0.0026 & 0.12 & $60 \mathrm{C}$ Aqueous Phase SCW \\
\hline CC8 066 & $\mathrm{C}_{4}$ & 4392 & 51.629 & 0.0030 & 0.13 & $60 \mathrm{C}$ Aqueous Phase SCW \\
\hline DCA 061 & $\mathrm{C} 22$ & 4392 & 52.388 & 0.0018 & 0.08 & 60 C Vapor Phase SCW \\
\hline DCA 062 & $\mathrm{C} 22$ & 4392 & 52.480 & 0.0022 & 0.10 & 60 C Vapor Phase SCW \\
\hline
\end{tabular}




\section{Corrosion Rate Calculations for Crevice Specimens}

For Rack Number : 27-1

$\begin{array}{lllll}\text { Sample Allov Lag Time Surface Area Weight Corrosion Rate Test Environment } \\ \text { Identification } & \text { (hours) } & \text { (sq cm) } & \text { Loss (g) (microns/year) }\end{array}$

\begin{tabular}{|c|c|c|c|c|c|c|}
\hline DCA 063 & $\mathrm{C} 22$ & 4392 & 52.372 & 0.0023 & 0.10 & $60 \mathrm{C}$ Vapor Phase SCW \\
\hline DCA 064 & $\mathrm{C} 22$ & 4392 & 52.069 & 0.0022 & 0.10 & $60 \mathrm{C}$ Aqueous Phase SCW \\
\hline DCA 065 & $\mathrm{C} 22$ & 4392 & 51.539 & 0.0024 & 0.11 & $60 \mathrm{C}$ Aqueous Phase SCW \\
\hline DCA 066 & $\mathrm{C} 22$ & 4392 & 52.605 & 0.0024 & 0.11 & $60 \mathrm{C}$ Aqueous Phase SCW \\
\hline DCB 061 & $\mathrm{C} 22$ & 4392 & 50.411 & 0.0012 & 0.06 & 60 C Vapor Phase SCW \\
\hline DCB 062 & $\mathrm{C} 22$ & 4392 & 51.690 & 0.0023 & 0.10 & 60 C Vapor Phase SCW \\
\hline DCB 063 & $\mathrm{C} 22$ & 4392 & 51.620 & 0.0025 & 0.11 & 60 C Vapor Phase SCW \\
\hline DCB 064 & $\mathrm{C} 22$ & 4392 & 50.133 & 0.0028 & 0.13 & $60 \mathrm{C}$ Aqueous Phase SCW \\
\hline DCB 065 & $\mathrm{C} 22$ & 4392 & 50.341 & 0.0020 & 0.09 & $60 \mathrm{C}$ Aqueous Phase SCW \\
\hline DCB 066 & $\mathrm{C} 22$ & 4392 & 50.409 & 0.0019 & 0.09 & $60 \mathrm{C}$ Aqueous Phase SCW \\
\hline ECA 061 & Ti Grade 12 & 4392 & 52.666 & 0.0948 & 8.10 & $60 \mathrm{C}$ Vapor Phase SCW \\
\hline ECA 062 & Ti Grade 12 & 4392 & 52.559 & 0.1051 & 9.00 & $60 \mathrm{C}$ Vapor Phase SCW \\
\hline ECA 063 & Ti Grade 12 & 4392 & 52.486 & 0.0789 & 6.77 & 60 C Vapor Phase SCW \\
\hline ECA 064 & Ti Grade 12 & 4392 & 51.722 & 0.0861 & 7.49 & $60 \mathrm{C}$ Aqueous Phase SCW \\
\hline ECA 065 & Ti Grade 12 & 4392 & 52.618 & 0.0897 & 7.68 & $60 \mathrm{C}$ Aqueous Phase SCW \\
\hline ECA 066 & Ti Grade 12 & 4392 & 51.504 & 0.0821 & 7.18 & $60 \mathrm{C}$ Aqueous Phase SCW \\
\hline ECD 061 & Ti Grade 12 & 4392 & 53.831 & 0.0884 & 7.39 & $60 \mathrm{C}$ Vapor Phase SCW \\
\hline ECD 062 & Ti Grade 12 & 4392 & 53.742 & 0.1104 & 9.25 & $60 \mathrm{C}$ Vapor Phase SCW \\
\hline ECD 063 & Ti Grade 12 & 4392 & 53.678 & 0.0684 & 5.74 & $60 \mathrm{C}$ Vapor Phase SCW \\
\hline
\end{tabular}




\section{Corrosion Rate Calculations for Crevice Specimens}

\section{For Rack Number : 27-1}

$\begin{array}{lrrr}\text { Sample Allov } & \text { Lag Time Surface Area Weight Corrosion Rate Test Environment } \\ \text { Identification } & \text { (hours) } & \text { (sq cm) } & \text { Loss (g) (microns/year) }\end{array}$

\begin{tabular}{|c|c|c|c|c|c|c|}
\hline ECD 064 & Ti Grade 12 & 4392 & 53.585 & 0.0937 & 7.87 & $60 \mathrm{C}$ Aqueous Phase SCW \\
\hline ECD 065 & Ti Grade 12 & 4392 & 53.867 & 0.0790 & 6.60 & $60 \mathrm{C}$ Aqueous Phase SCW \\
\hline ECD 066 & Ti Grade 12 & 4392 & 53.691 & 0.0815 & 6.83 & $60 \mathrm{C}$ Aqueous Phase SCW \\
\hline FCA 061 & Ti Grade 16 & 4392 & 52.379 & 0.0674 & 5.68 & $60 \mathrm{C}$ Vapor Phase SCW \\
\hline FCA 062 & Ti Grade 16 & 4392 & 52.375 & 0.0582 & 4.90 & 60 C Vapor Phase SCW \\
\hline FCA 063 & Ti Grade 16 & 4392 & 52.323 & 0.0563 & 4.75 & $60 \mathrm{C}$ Vapor Phase SCW \\
\hline FCA 064 & Ti Grade 16 & 4392 & 52.411 & 0.0589 & 4.96 & $60 \mathrm{C}$ Aqueous Phase SCW \\
\hline FCA 065 & Ti Grade 16 & 4392 & 52.901 & 0.0442 & 3.69 & $60 \mathrm{C}$ Aqueous Phase SCW \\
\hline FCA 066 & Ti Grade 16 & 4392 & 52.274 & 0.0559 & 4.72 & $60 \mathrm{C}$ Aqueous Phase SCW \\
\hline FCE 061 & Ti Grade 16 & 4392 & 51.540 & 0.2982 & 25.53 & $60 \mathrm{C}$ Vapor Phase SCW \\
\hline FCE 062 & Ti Grade 16 & 4392 & 51.446 & -0.1295 & -11.11 & $60 \mathrm{C}$ Vapor Phase SCW \\
\hline FCE 063 & Ti Grade 16 & 4392 & 51.057 & 0.0429 & 3.71 & 60 C Vapor Phase SCW \\
\hline FCE 064 & Ti Grade 16 & 4392 & 51.272 & 0.0398 & 3.43 & $60 \mathrm{C}$ Aqueous Phase SCW \\
\hline FCE 065 & Ti Grade 16 & 4392 & 51.174 & 0.0411 & 3.54 & $60 \mathrm{C}$ Aqueous Phase SCW \\
\hline FCE 066 & Ti Grade 16 & 4392 & 51.104 & 0.0513 & 4.43 & $60 \mathrm{C}$ Aqueous Phase SCW \\
\hline LCA 061 & Inconel 625 & 4392 & 52.229 & 0.0012 & 0.05 & $60 \mathrm{C}$ Vapor Phase SCW \\
\hline LCA 062 & Inconel 625 & 4392 & 51.787 & 0.0007 & 0.03 & $60 \mathrm{C}$ Vapor Phase SCW \\
\hline LCA 063 & Inconel 625 & 4392 & 51.765 & 0.0013 & 0.06 & $60 \mathrm{C}$ Vapor Phase SCW \\
\hline LCA 064 & Inconel 625 & 4392 & 52.215 & 0.0015 & 0.07 & $60 \mathrm{C}$ Aqueous Phase SCW \\
\hline
\end{tabular}




\section{Corrosion Rate Calculations for Crevice Specimens}

For Rack Number : 27-1

$\begin{array}{llrll}\text { Sample Allov Lag Time Surface Area } & \text { Weight Corrosion Rate } & \text { Test Environment } \\ \text { Identification } & \text { (hours) } & \text { (sq cm) } & \text { Loss (g) (microns/year) }\end{array}$

\begin{tabular}{lllllll}
\hline LCA 065 & Inconel 625 & 4392 & 52.221 & 0.0011 & 0.05 & $60 \mathrm{C}$ Aqueous Phase SCW \\
LCA 066 & Inconel 625 & 4392 & 52.440 & 0.0018 & 0.08 & $60 \mathrm{C}$ Aqueous Phase SCW \\
LCJ 061 & Inconel 625 & 4392 & 51.336 & 0.0006 & 0.03 & $60 \mathrm{C}$ Vapor Phase SCW \\
LCJ 062 & Inconel 625 & 4392 & 50.854 & 0.0021 & 0.10 & $60 \mathrm{C}$ Vapor Phase SCW \\
LCJ 063 & Inconel 625 & 4392 & 51.136 & 0.0005 & 0.02 & $60 \mathrm{C}$ Vapor Phase SCW \\
LCJ 064 & Inconel 625 & 4392 & 51.103 & 0.0017 & 0.08 & $60 \mathrm{C}$ Aqueous Phase SCW \\
LCJ 065 & Inconel 625 & 4392 & 51.355 & 0.0014 & 0.06 & $60 \mathrm{C}$ Aqueous Phase SCW \\
LCJ 066 & Inconel 625 & 4392 & 51.268 & 0.0022 & 0.10 & $60 \mathrm{C}$ Aqueous Phase SCW
\end{tabular}




\section{Corrosion Rate Calculations for Crevice Specimens}

For Rack Number : 28-1

Sample Allov Lag Time Surface Area Weight Corrosion Rate Test Environment Identification (hours) (sq cm) Loss (g) (microns/year)

\begin{tabular}{|c|c|c|c|c|c|c|}
\hline ACA 091 & 1825 & 4464 & 52.425 & 0.0017 & 0.08 & 90 C Vapor Phase SCW \\
\hline ACA 092 & 1825 & 4464 & 51.837 & 0.0015 & 0.07 & $90 \mathrm{C}$ Vapor Phase SCW \\
\hline ACA 093 & 1825 & 4464 & 51.983 & 0.0017 & 0.08 & $90 \mathrm{C}$ Vapor Phase SCW \\
\hline ACA 094 & 1825 & 4464 & 51.999 & 0.0026 & 0.12 & $90 \mathrm{C}$ Aqueous Phase SCW \\
\hline ACA 095 & 1825 & 4464 & 52.631 & 0.0023 & 0.11 & $90 \mathrm{C}$ Aqueous Phase SCW \\
\hline ACA 096 & 1825 & 4464 & 51.775 & 0.0015 & 0.07 & $90 \mathrm{C}$ Aqueous Phase SCW \\
\hline ACB 091 & 1825 & 4464 & 51.609 & 0.0014 & 0.07 & $90 \mathrm{C}$ Vapor Phase SCW \\
\hline АСВ 092 & 1825 & 4464 & 52.166 & 0.0013 & 0.06 & $90 \mathrm{C}$ Vapor Phase SCW \\
\hline ACB 093 & 1825 & 4464 & 51.599 & 0.0017 & 0.08 & $90 \mathrm{C}$ Vapor Phase SCW \\
\hline ACB 094 & 1825 & 4464 & 52.293 & 0.0020 & 0.09 & $90 \mathrm{C}$ Aqueous Phase SCW \\
\hline ACB 095 & 1825 & 4464 & 52.149 & 0.0025 & 0.12 & $90 \mathrm{C}$ Aqueous Phase SCW \\
\hline АСВ 096 & 1825 & 4464 & 52.185 & 0.0022 & 0.10 & $90 \mathrm{C}$ Aqueous Phase SCW \\
\hline BCA 091 & G3 & 4464 & 52.205 & 0.0006 & 0.03 & 90 C Vapor Phase SCW \\
\hline BCA 092 & $\mathbf{G 3}$ & 4464 & 52.134 & 0.0010 & 0.05 & 90 C Vapor Phase SCW \\
\hline BCA 093 & G3 & 4464 & 52.360 & 0.0004 & 0.02 & $90 \mathrm{C}$ Vapor Phase SCW \\
\hline BCA 094 & G3 & 4464 & 52.294 & 0.0019 & 0.09 & $90 \mathrm{C}$ Aqueous Phase SCW \\
\hline BCA 095 & G3 & 4464 & 52.563 & 0.0027 & 0.12 & $90 \mathrm{C}$ Aqueous Phase SCW \\
\hline BCA 096 & G3 & 4464 & 52.514 & 0.0016 & 0.07 & $90 \mathrm{C}$ Aqueous Phase SCW \\
\hline BCC 091 & G3 & 4464 & 52.043 & 0.0010 & 0.05 & $90 \mathrm{C}$ Vapor Phase SCW \\
\hline
\end{tabular}




\section{Corrosion Rate Calculations for Crevice Specimens}

For Rack Number : 28-1

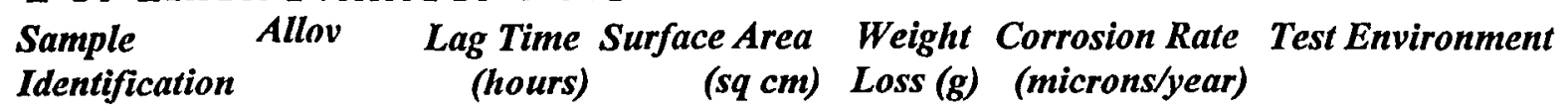

\begin{tabular}{|c|c|c|c|c|c|c|}
\hline BCC 092 & G3 & 4464 & 53.369 & 0.0007 & 0.03 & $90 \mathrm{C}$ Vapor Phase SCW \\
\hline BCC 093 & G3 & 4464 & 52.560 & 0.0008 & 004 & $90 \mathrm{C}$ Vapor Phase SCW \\
\hline BCC 094 & G3 & 4464 & 52.024 & 0.0029 & 0.13 & $90 \mathrm{C}$ Aqueous Phase SCW \\
\hline BCC 095 & G3 & 4464 & 52,800 & 0.0028 & 0.13 & $90 \mathrm{C}$ Aqueous Phase SCW \\
\hline BCC 096 & G3 & 4464 & 50.965 & 0.0036 & 0.17 & $90 \mathrm{C}$ Aqueous Phase SCW \\
\hline CCA 091 & $\mathrm{C} 4$ & 4464 & 52.428 & 0.0007 & 0.03 & 90 C Vapor Phase SCW \\
\hline CCA 092 & $\mathrm{C4}$ & 4464 & 52.223 & 0.0004 & 0.02 & 90 C Vapor Phase SCW \\
\hline CCA 093 & $\mathrm{C} 4$ & 4464 & 52.320 & 0.0010 & 0.04 & $90 \mathrm{C}$ Vapor Phase SCW \\
\hline CCA 094 & $\mathrm{C} 4$ & 4464 & 52.307 & 0.0026 & 0.11 & $90 \mathrm{C}$ Aqueous Phase SCW \\
\hline CCA 095 & $\mathrm{C} 4$ & 4464 & 52.428 & 0.0031 & 0.13 & $90 \mathrm{C}$ Aqueous Phase SCW \\
\hline CCA 096 & $\mathrm{C} 4$ & 4464 & 52.406 & 0.0028 & 0.12 & $90 \mathrm{C}$ Aqueous Phase SCW \\
\hline ССВ 091 & C4 & 4464 & 51.254 & 0.0005 & 0.02 & $90 \mathrm{C}$ Vapor Phase SCW \\
\hline CCB 092 & $\mathrm{C}_{4}$ & 4464 & 51.442 & 0.0002 & 0.01 & $90 \mathrm{C}$ Vapor Phase SCW \\
\hline ССВ 093 & $\mathrm{C4}$ & 4464 & 51.547 & 0.0009 & 0.04 & 90 C Vapor Phase SCW \\
\hline ССВ 094 & $\mathrm{C}_{4}$ & 4464 & 51.590 & 0.0024 & 0.11 & $90 \mathrm{C}$ Aqueous Phase SCW \\
\hline ССВ 095 & $\mathrm{C4}$ & 4464 & 51.748 & 0.0032 & 0.14 & $90 \mathrm{C}$ Aqueous Phase SCW \\
\hline CСB 096 & $\mathrm{C} 4$ & 4464 & 51.369 & 0.0038 & 0.17 & $90 \mathrm{C}$ Aqueous Phase SCW \\
\hline DCA 091 & $\mathrm{C} 22$ & 4464 & 52.853 & 0.0016 & 0.07 & 90 C Vapor Phase SCW \\
\hline DCA 092 & $\mathrm{C} 22$ & 4464 & 51.954 & 0.0011 & 0.05 & 90 C Vapor Phase SCW \\
\hline
\end{tabular}




\section{Corrosion Rate Calculations for Crevice Specimens}

For Rack Number : 28-1 $\begin{array}{llll}\text { Sample Allov Lag Time Surface Area } & \text { Weight Corrosion Rate Test Environment } \\ \text { Identification } & \text { (hours) } & \text { (sq cm) Loss }(\mathrm{g}) \text { (microns/year) }\end{array}$

\begin{tabular}{|c|c|c|c|c|c|c|}
\hline DCA 093 & $\mathrm{C} 22$ & 4464 & 52.738 & 0.0108 & 0.47 & $90 \mathrm{C}$ Vapor Phase SCW \\
\hline DCA 094 & $\mathrm{C} 22$ & 4464 & 52410 & 0.0023 & 0.10 & $90 \mathrm{C}$ Aqueous Phase SCW \\
\hline DCA 095 & $\mathrm{C} 22$ & 4464 & 52.396 & 0.0015 & 0.07 & $90 \mathrm{C}$ Aqueous Phase SCW \\
\hline DCA 096 & $\mathrm{C} 22$ & 4464 & 52.628 & 0.0033 & 0.14 & $90 \mathrm{C}$ Aqueous Phase SCW \\
\hline DCB 091 & $\mathrm{C} 22$ & 4464 & 51.482 & 0.0000 & 0.00 & $90 \mathrm{C}$ Vapor Phase SCW \\
\hline DCB 092 & $\mathrm{C} 22$ & 4464 & 51.394 & 0.0006 & 0.03 & $90 \mathrm{C}$ Vapor Phase SCW \\
\hline DCB 093 & $\mathrm{C} 22$ & 4464 & 51.210 & 0.0009 & 0.04 & $90 \mathrm{C}$ Vapor Phase SCW \\
\hline DCB 094 & $\mathrm{C} 22$ & 4464 & 51.110 & 0.0015 & 0.07 & $90 \mathrm{C}$ Aqueous Phase SCW \\
\hline DCB 095 & $\mathrm{C} 22$ & 4464 & 51.910 & 0.0057 & 0.25 & $90 \mathrm{C}$ Aqueous Phase SCW \\
\hline DCB 096 & $\mathrm{C} 22$ & 4464 & 51.529 & 0.0023 & 0.10 & $90 \mathrm{C}$ Aqueous Phase SCW \\
\hline ECA 091 & Ti Grade 12 & 4464 & 52.764 & 0.1209 & 10.15 & $90 \mathrm{C}$ Vapor Phase SCW \\
\hline ECA 092 & Ti Grade 12 & 4464 & 52.768 & 0.0921 & 7.73 & $90 \mathrm{C}$ Vapor Phase SCW \\
\hline ECA 093 & Ti Grade 12 & 4464 & 52.758 & 0.0739 & 6.20 & $90 \mathrm{C}$ Vapor Phase SCW \\
\hline ECA 094 & Ti Grade 12 & 4464 & 52.279 & 0.4216 & 35.72 & $90 \mathrm{C}$ Aqueous Phase SCW \\
\hline ECA 095 & Ti Grade 12 & 4464 & 51.135 & 0.0804 & 6.96 & $90 \mathrm{C}$ Aqueous Phase SCW \\
\hline ECA 096 & Ti Grade 12 & 4464 & 51.859 & 0.0901 & 7.70 & $90 \mathrm{C}$ Aqueous Phase SCW \\
\hline ECD 091 & Ti Grade 12 & 4464 & 52.792 & 0.0696 & 5.84 & $90 \mathrm{C}$ Vapor Phase SCW \\
\hline ECD 092 & Ti Grade 12 & 4464 & 53.930 & 0.0682 & 5.60 & 90 C Vapor Phase SCW \\
\hline ECD 093 & $\pi$ Grade 12 & 4464 & 53.833 & 0.0962 & 7.90 & 80 C Vapor Phase SCW \\
\hline
\end{tabular}




\section{Corrosion Rate Calculations for Crevice Specimens}

For Rack Number : 28-1

$\begin{array}{llll}\text { Sample Allov Lag Time Surface Area Weight Corrosion Rate Test Environment } \\ \text { Identification } & \text { (hours) } & \text { (sq cm) Loss }(g) \text { (microns/year) }\end{array}$

\begin{tabular}{|c|c|c|c|c|c|c|}
\hline ECD 094 & Ti Grade 12 & 4464 & 54.036 & 0.0842 & 6.90 & $90 \mathrm{C}$ Aqueous Phase SCW \\
\hline ECD 095 & Ti Grade 12 & 4464 & 53490 & 0.0995 & 8.24 & $90 \mathrm{C}$ Aqueous Phase SCW \\
\hline ECD 096 & Ti Grade 12 & 4464 & 53.710 & 0.0939 & 7.74 & $90 \mathrm{C}$ Aqueous Phase SCW \\
\hline FCA 091 & Ti Grade 16 & 4464 & 52.637 & 0.0902 & 7.44 & $90 \mathrm{C}$ Vapor Phase SCW \\
\hline FCA 092 & Ti Grade 16 & 4464 & 52.871 & 0.1321 & 10.85 & $90 \mathrm{C}$ Vapor Phase SCW \\
\hline FCA 093 & Ti Grade 16 & 4464 & 52.592 & 0.1158 & 9.56 & $90 \mathrm{C}$ Vapor Phase SCW \\
\hline FCA 094 & Ti Grade 16 & 4464 & 52.891 & 0.0800 & 6.57 & $90 \mathrm{C}$ Aqueous Phase SCW \\
\hline FCA 095 & Ti Grade 16 & 4464 & 52.427 & 0.0811 & 6.72 & $90 \mathrm{C}$ Aqucous Phase SCW \\
\hline FCA 096 & Ti Grade 16 & 4464 & 52.494 & 0.1044 & 8.63 & $90 \mathrm{C}$ Aqueous Phase SCW \\
\hline FCE 091 & Ti Grade 16 & 4464 & 50.651 & 0.0812 & 6.96 & $90 \mathrm{C}$ Vapor Phase SCW \\
\hline FCE 092 & Ti Grade 16 & 4464 & 50.942 & 0.0874 & 7.45 & 90 C Vapor Phase SCW \\
\hline FCE 093 & Ti Grade 16 & 4464 & 51.621 & 0.0791 & 6.65 & 90 C Vapor Phase SCW \\
\hline FCE 094 & Ti Grade 16 & 4464 & 51.387 & 0.0742 & 6.27 & $90 \mathrm{C}$ Aqueous Phase SCW \\
\hline FCE 095 & Ti Grade 16 & 4464 & 51.133 & 0.0625 & 5.31 & $90 \mathrm{C}$ Aqueous Phase SCW \\
\hline FCE 096 & Ti Grade 16 & 4464 & 51.559 & 0.0706 & 5.94 & $90 \mathrm{C}$ Aqueous Phase SCW \\
\hline LCA 091 & Inconel 625 & 4464 & 51.107 & 0.0004 & 0.02 & 90 C Vapor Phase SCW \\
\hline LCA 092 & Inconel 625 & 4464 & 52.109 & 0.0002 & 0.01 & $90 \mathrm{C}$ Vapor Phase SCW \\
\hline LCA 093 & Inconel 625 & 4464 & 52.367 & 0.0011 & 0.05 & 90 C Vapor Phase SCW \\
\hline LCA 094 & Inconel 625 & 4464 & 52.709 & 0.0025 & 0.11 & $90 \mathrm{C}$ Aqueous Phase SCW \\
\hline
\end{tabular}




\section{Corrosion Rate Calculations for Crevice Specimens}

For Rack Number : 28-1

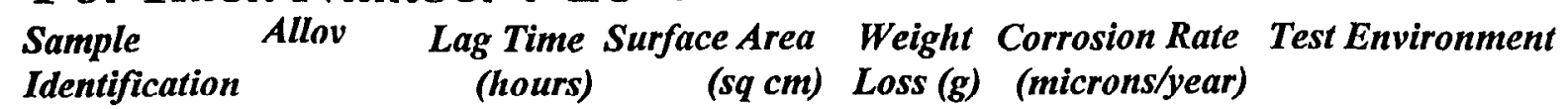

\begin{tabular}{lllllll}
\hline LCA 095 & Inconel 625 & 4464 & 52.492 & 0.0022 & 0.10 & $90 \mathrm{C}$ Aqueous Phase SCW \\
LCA 096 & Inconel 625 & 4464 & 52.405 & 0.0055 & 0.24 & $90 \mathrm{C}$ Aqueous Phase SCW \\
LCJ 091 & Inconel 625 & 4464 & 51.379 & 0.0002 & 0.01 & $90 \mathrm{C}$ Vapor Phase SCW \\
LCJ 092 & Inconel 625 & 4464 & 51.497 & 0.0006 & 0.03 & $90 \mathrm{C}$ Vapor Phase SCW \\
LCJ 093 & Inconel 625 & 4464 & 51.340 & 0.0004 & 0.02 & $90 \mathrm{C}$ Vapor Phase SCW \\
LCJ 094 & Inconel 625 & 4464 & 51.468 & 0.0013 & 0.06 & $90 \mathrm{C}$ Aqueous Phase SCW \\
LCJ 095 & Inconel 625 & 4464 & 51.468 & 0.0015 & 0.07 & $90 \mathrm{C}$ Aqueous Phase SCW \\
LCJ 096 & Inconel 625 & 4464 & 51.205 & 0.0026 & 0.12 & $90 \mathrm{C}$ Aqueous Phase SCW
\end{tabular}




\section{Corrosion Rate Calculations for Crevice Specimens}

\section{For Rack Number : 29-1}

\section{Sample Allov Lag Time Surface Area Weight Corrosion Rate Test Environment Identification (hours) ( $\mathrm{sq} \mathrm{cm})$ Loss (g) (microns/year)}

\begin{tabular}{|c|c|c|c|c|c|c|}
\hline ACA 121 & 1825 & 4464 & 51925 & 0.0021 & 010 & $60 \mathrm{C}$ Vapor Phase SDW \\
\hline ACA 122 & 1825 & 4464 & 52.436 & 0.0023 & 011 & $60 \mathrm{C}$ Vapor Phase SDW \\
\hline ACA 123 & 1825 & 4464 & 52.459 & 0.0014 & 0.06 & $60 \mathrm{C}$ Vapor Phase SDW \\
\hline ACA 124 & 1825 & 4464 & 52.497 & 0.0016 & 0.07 & $60 \mathrm{C}$ Aqueous Phase SDW \\
\hline ACA 125 & 1825 & 4464 & 52.206 & 0.0022 & 0.10 & $60 \mathrm{C}$ Aqueous Phase SDW \\
\hline ACA 126 & 1825 & 4464 & 52.536 & 0.0019 & 0.09 & $60 \mathrm{C}$ Aqueous Phase SDW \\
\hline ACB 121 & 1825 & 4464 & 52.060 & 0.0014 & 0.06 & $60 \mathrm{C}$ Vapor Phase SDW \\
\hline ACB 122 & 1825 & 4464 & 51.580 & 0.0015 & 0.07 & $60 \mathrm{C}$ Vapor Phase SDW \\
\hline ACB 123 & 1825 & 4464 & 51.584 & 0.0020 & 0.09 & $60 \mathrm{C}$ Vapor Phase SDW \\
\hline ACB 124 & 1825 & 4464 & 51.360 & 0.0018 & 0.08 & $60 \mathrm{C}$ Aqueous Phase SDW \\
\hline ACB 125 & 1825 & 4464 & 51.734 & 0.0014 & 0.07 & $60 \mathrm{C}$ Aqueous Phase SDW \\
\hline АСВ 126 & 1825 & 4464 & 51.718 & 0.0015 & 0.07 & $60 \mathrm{C}$ Aqueous Phase SDW \\
\hline BCA 121 & G3 & 4464 & 51.883 & 0.0015 & 0.07 & $60 \mathrm{C}$ Vapor Phase SDW \\
\hline BCA 122 & G3 & 4464 & 52.562 & 0.0025 & 0.11 & 60 C Vapor Phase SDW \\
\hline BCA 123 & G3 & 4464 & 51.846 & 0.0016 & 0.07 & 60 C Vapor Phase SDW \\
\hline BCA 124 & G3 & 4464 & 52.411 & 0.0010 & 0.05 & $60 \mathrm{C}$ Aqueous Phase SDW \\
\hline BCA 125 & G3 & 4464 & 51.997 & 0.0016 & 0.07 & $60 \mathrm{C}$ Aqueous Phase SDW \\
\hline BCA 126 & G3 & 4464 & 52.453 & 0.0017 & 0.08 & $60 \mathrm{C}$ Aqueous Phase SDW \\
\hline BCC 121 & G3 & 4464 & 52.438 & 0.0013 & 0.06 & $60 \mathrm{C}$ Vapar Phase SDW \\
\hline
\end{tabular}




\section{Corrosion Rate Calculations for Crevice Specimens}

For Rack Number : 29-1 $\begin{array}{llll}\text { Sample Allov Lag Time Surface Area Weight Corrosion Rate Test Environment } \\ \text { Identification } & \text { (hours) } & \text { (sq cm) Lass (g) (microns/year) }\end{array}$

\begin{tabular}{|c|c|c|c|c|c|c|}
\hline BCC 122 & G3 & 4464 & 51.838 & 0.0009 & 0.04 & $60 \mathrm{C}$ Vapor Phase SDW \\
\hline BCC 123 & G3 & 4464 & 52.324 & 0.0017 & 0.08 & $60 \mathrm{C}$ Vapor Phase SDW \\
\hline BCC 124 & G3 & 4464 & 52.207 & 0.0012 & 0.05 & $60 \mathrm{C}$ Aqueous Phase SDW \\
\hline BCC 125 & G3 & 4464 & 52.186 & 0.0017 & 0.08 & $60 \mathrm{C}$ Aqueous Phase SDW \\
\hline BCC 126 & G3 & 4464 & 52.593 & 0.0013 & 0.06 & $60 \mathrm{C}$ Aqueous Phase SOW \\
\hline CCA 121 & $\mathrm{C4}$ & 4464 & 52.450 & 0.0011 & 0.05 & $60 \mathrm{C}$ Vapor Phase SDW \\
\hline CCA 122 & $\mathrm{C4}$ & 4464 & 51.972 & 0.0007 & 0.03 & $60 \mathrm{C}$ Vapor Phase SDW \\
\hline $\operatorname{CCA} 123$ & $\mathrm{C} 4$ & 4464 & 52.177 & 0.0017 & 0.07 & $60 \mathrm{C}$ Vapor Phase SDW \\
\hline CCA 124 & $\mathrm{C4}$ & 4464 & 52.139 & 0.0009 & 0.04 & $60 \mathrm{C}$ Aqueous Phase SDW \\
\hline CCA 125 & $\mathrm{C} 4$ & 4464 & 52.349 & 0.0014 & 0.06 & $60 \mathrm{C}$ Aqueous Phase SDW \\
\hline CCA 126 & $\mathrm{C} 4$ & 4464 & 52.205 & 0.0008 & 0.03 & $60 \mathrm{C}$ Aqueous Phase SDW \\
\hline CCB 121 & $\mathrm{C4}$ & 4464 & 51.659 & 0.0012 & 0.05 & 60 C Vapor Phase SDW \\
\hline CCB 122 & C4 & 4464 & 50.701 & 0.0009 & 0.04 & $60 \mathrm{C}$ Vapor Phase SDW \\
\hline CCB 123 & $\mathrm{C}_{4}=$ & 4464 & 51.236 & 0.0014 & 0.06 & $60 \mathrm{C}$ Vapor Phase SDW \\
\hline CCB 124 & $\mathrm{C4}$ & 4464 & 51.731 & 0.0016 & 0.07 & $60 \mathrm{C}$ Aqueous Phase SDW \\
\hline ССВ 125 & $\mathrm{C} 4$ & 4464 & 51.464 & 0.0013 & 0.06 & $60 \mathrm{C}$ Aqueous Phase SDW \\
\hline ССВ 126 & C4 & 4464 & 51.539 & 0.0010 & 0.04 & $60 \mathrm{C}$ Aqueous Phase SDW \\
\hline DCA 121 & $\mathrm{C} 22$ & 4464 & 52.491 & 0.0022 & 0.10 & $60 \mathrm{C}$ Vapor Phase SDW \\
\hline DCA 122 & $\mathrm{C} 22$ & 4464 & 52.547 & 0.0017 & 0.07 & 60 C Vapor Phase SOW \\
\hline
\end{tabular}




\section{Corrosion Rate Calculations for Crevice Specimens For Rack Number : 29-1}

Sample Allov Lag Time Surface Area Weight Corrosion Rate Test Environment Identification (hours) (sq cm) Loss (g) (microns/year)

\begin{tabular}{|c|c|c|c|c|c|c|}
\hline DCA 123 & $\mathrm{C} 22$ & 4464 & 52.639 & 0.0020 & 0.09 & 60 C Vapor Phase SDW \\
\hline DCA 124 & $\mathrm{C} 22$ & 4464 & 52.688 & 0.0020 & 0.09 & $60 \mathrm{C}$ Aqueous Phase SDW \\
\hline DCA 125 & $\mathrm{C} 22$ & 4464 & 52.558 & 0.0015 & 0.07 & $60 \mathrm{C}$ Aqueous Phase SDW \\
\hline DCA 126 & $\mathrm{C} 22$ & 4464 & 52.651 & 0.0013 & 0.06 & $60 \mathrm{C}$ Aqueous Phase SDW \\
\hline DCB 121 & C22 & 4464 & 50.843 & 0.0016 & 0.07 & $60 \mathrm{C}$ Vapor Phase SDW \\
\hline DCB 122 & $\mathrm{C} 22$ & 4464 & 50.829 & 0.0019 & 0.09 & $60 \mathrm{C}$ Vapor Phase SDW \\
\hline DCB 123 & $\mathrm{C} 22$ & 4464 & 50.970 & 0.0020 & 0.09 & $60 \mathrm{C}$ Vapor Phase SDW \\
\hline DCB 124 & $\mathrm{C} 22$ & 4464 & 50.808 & 0.0022 & 0.10 & $60 \mathrm{C}$ Aqueous Phase SDW \\
\hline DCB 125 & $\mathrm{C} 22$ & 4464 & 50.007 & 0.0016 & 0.07 & $60 \mathrm{C}$ Aqueous Phase SDW \\
\hline DCB 126 & $\mathrm{C} 22$ & 4464 & 51.726 & 0.0023 & 0.10 & $60 \mathrm{C}$ Aqueous Phase SDW \\
\hline ECA 121 & Ti Grade 12 & 4464 & 53.598 & 0.0721 & 5.96 & $60 \mathrm{C}$ Vapor Phase SDW \\
\hline ECA 122 & Ti Grade 12 & 4464 & 53.869 & 0.0551 & 4.53 & $60 \mathrm{C}$ Vapor Phase SDW \\
\hline ECA 123 & Ti Grade 12 & 4464 & 53.583 & 0.0493 & 4.08 & $60 \mathrm{C}$ Vapor Phase SDW \\
\hline ECA 124 & Ti Grade 12 & 4464 & 53.085 & 0.0677 & 5.65 & $60 \mathrm{C}$ Aqueous Phase SDW \\
\hline ECA 125 & Ti Grade 12 & 4464 & 53.491 & 0.0538 & 4.46 & $60 \mathrm{C}$ Aqueous Phase SDW \\
\hline ECA 126 & Ti Grade 12 & 4464 & 53.664 & 0.0686 & 5.66 & $60 \mathrm{C}$ Aqueous Phase SDW \\
\hline ECD 121 & Ti Grade 12 & 4464 & 53.704 & 0.0619 & 5.11 & $60 \mathrm{C}$ Vapor Phase SDW \\
\hline ECD 122 & Ti Grade 12 & 4464 & 54.163 & 0.0685 & 5.60 & 60 C Vapor Phase SDW \\
\hline ECD 123 & Ti Grade 12 & 4464 & 53.964 & 0.0966 & 7.93 & $60 \mathrm{C}$ Vapor Phase SDW \\
\hline
\end{tabular}




\section{Corrosion Rate Calculations for Crevice Specimens}

For Rack Number : 29-1

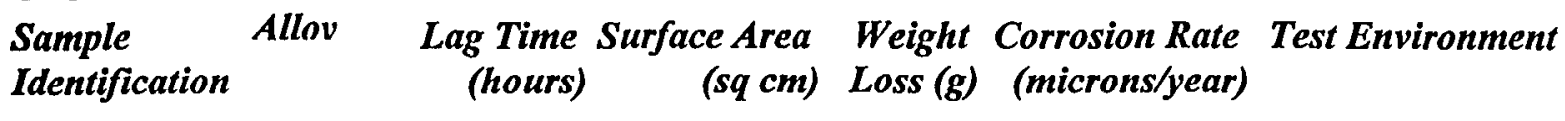

\begin{tabular}{|c|c|c|c|c|c|c|}
\hline ECD 124 & Ti Grade 12 & 4464 & 53.362 & 0.0796 & 6.61 & $60 \mathrm{C}$ Aqueous Phase SDW \\
\hline ECD 125 & Ti Grade 12 & 4464 & 53.983 & 0.0642 & 5.27 & $60 \mathrm{C}$ Aqueous Phase SDW \\
\hline ECD 126 & Ti Grade 12 & 4464 & 53.837 & 0.0567 & 4.67 & $60 \mathrm{C}$ Aqueous Phase SDW \\
\hline FCA 121 & Ti Grade 16 & 4464 & 52.824 & 0.0567 & 4.66 & $60 \mathrm{C}$ Vapor Phase SDW \\
\hline FCA 122 & Ti Grade 16 & 4464 & 52.519 & 0.0535 & 4.42 & $60 \mathrm{C}$ Vapor Phase SDW \\
\hline FCA 123 & Ti Grade 16 & 4464 & 52.824 & 0.0725 & 5.96 & $60 \mathrm{C}$ Vapor Phase SDW \\
\hline FCA 124 & Ti Grade 16 & 4464 & 52.656 & 0.0572 & 4.72 & $60 \mathrm{C}$ Aqueous Phase SDW \\
\hline FCA 125 & Ti Grade 16 & 4464 & 53.162 & 0.0634 & 5.18 & $60 \mathrm{C}$ Aqueous Phase SDW \\
\hline FCA 126 & Ti Grade 16 & 4464 & 52.814 & 0.0628 & 5.16 & $60 \mathrm{C}$ Aqueous Phase SDW \\
\hline FCE 121 & Ti Grade 16 & 4464 & 51.418 & 0.0686 & 5.79 & $60 \mathrm{C}$ Vapor Phase SDW \\
\hline FCE 122 & Ti Grade 16 & 4464 & 51.509 & 0.0677 & 5.71 & $60 \mathrm{C}$ Vapor Phase SDW \\
\hline FCE 123 & Ti Grade 16 & 4464 & 51.897 & 0.0663 & 5.55 & $60 \mathrm{C}$ Vapor Phase SDW \\
\hline FCE 124 & Ti Grade 16 & 4464 & 51.255 & 0.0692 & 5.86 & $60 \mathrm{C}$ Aqueous Phase SDW \\
\hline FCE 125 & TI Grade 16 & 4464 & 51.214 & 0.0457 & 3.87 & $60 \mathrm{C}$ Aqueous Phase SDW \\
\hline FCE 126 & Ti Grade 16 & 4464 & 51.443 & 0.0508 & 4.29 & $60 \mathrm{C}$ Aqueous Phase SDW \\
\hline LCA 121 & Inconel 625 & 4464 & 51.793 & 0.0004 & 0.02 & $60 \mathrm{C}$ Vapor Phase SDW \\
\hline LCA 122 & Inconel 625 & 4464 & 52.048 & 0.0001 & 0.00 & $60 \mathrm{C}$ Vapor Phase SDW \\
\hline LCA 123 & Inconel 625 & 4464 & 52.116 & 0.0004 & 0.02 & $60 \mathrm{C}$ Vapor Phase SDW \\
\hline LCA 124 & Inconel 625 & 4464 & 52.116 & 0.0003 & 0.01 & $60 \mathrm{C}$ Aqueous Phase SDW \\
\hline
\end{tabular}




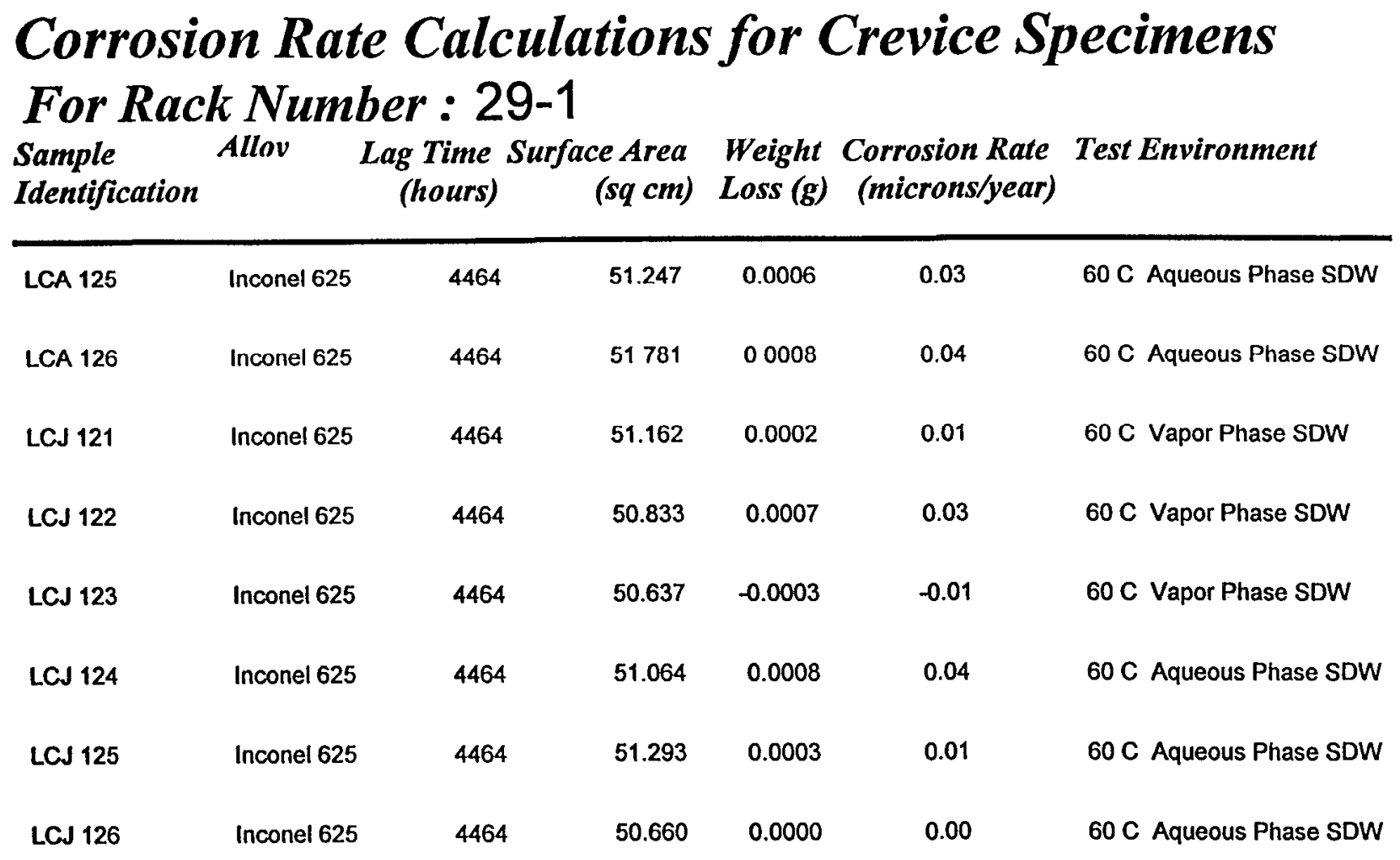




\section{Corrosion Rate Calculations for Crevice Specimens}

For Rack Number : 30-1

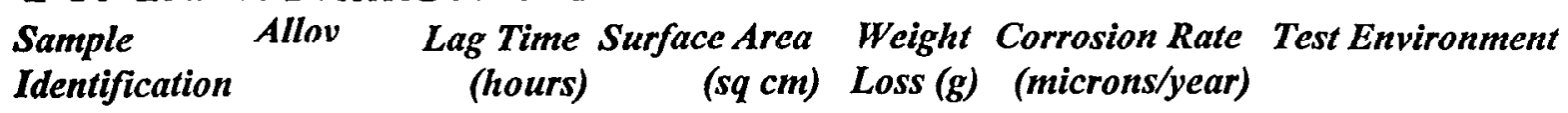

\begin{tabular}{|c|c|c|c|c|c|c|}
\hline ACA 157 & 1825 & 4392 & 52.318 & 00011 & 0.05 & $90 \mathrm{C}$ Vapor Phase SDW \\
\hline ACA 158 & 1825 & 4392 & 52.403 & 00012 & 0.06 & $90 \mathrm{C}$ Vapor Phase SDW \\
\hline ACA 159 & 1825 & 4392 & 52.643 & 0.0018 & 0.08 & $90 \mathrm{C}$ Vapor Phase SDW \\
\hline ACA 160 & 1825 & 4392 & 52.389 & 0.0010 & 0.05 & $90 \mathrm{C}$ Aqueous Phase SDW \\
\hline ACA 161 & 1825 & 4392 & 52.355 & 0.0018 & 0.08 & $90 \mathrm{C}$ Aqueous Phase SDW \\
\hline ACA 162 & 1825 & 4392 & 52.781 & 0.0028 & 0.13 & $90 \mathrm{C}$ Aqueous Phase SDW \\
\hline ACB 157 & 1825 & 4392 & 52.365 & 0.0010 & 0.05 & $90 \mathrm{C}$ Vapor Phase SDW \\
\hline АCB 158 & 1825 & 4392 & 51.704 & 0.0010 & 0.05 & $90 \mathrm{C}$ Vapor Phase SDW \\
\hline ACB 159 & 1825 & 4392 & 50.703 & 0.0011 & 0.05 & 90 C Vapor Phase SDW \\
\hline ACB 160 & 1825 & 4392 & 51.192 & 0.0012 & 0.06 & $90 \mathrm{C}$ Aqueous Phase SDW \\
\hline ACB 161 & 1825 & 4392 & 52.810 & 0.0012 & 0.06 & $90 \mathrm{C}$ Aqueous Phase SDW \\
\hline ACB 162 & 1825 & 4392 & 52.633 & 0.0007 & 0.03 & $90 \mathrm{C}$ Aqueous Phase SDW \\
\hline BCA 157 & G3 & 4392 & 51.950 & 0.0012 & 0.06 & $90 \mathrm{C}$ Vapor Phase SDW \\
\hline BCA 158 & G3 & 4392 & 51.867 & 0.0021 & 0.10 & $90 \mathrm{C}$ Vapor Phase SDW \\
\hline BCA 159 & G3 & 4392 & 52.116 & 0.0018 & 0.08 & $90 \mathrm{C}$ Vapor Phase SDW \\
\hline BCA 160 & G3 & 4392 & 52.290 & 0.0019 & 0.09 & $90 \mathrm{C}$ Aqueous Phase SDW \\
\hline BCA 161 & G3 & 4392 & 52.202 & 0.0020 & 0.09 & $90 \mathrm{C}$ Aqueous Phase SDW \\
\hline BCA 162 & G3 & 4392 & 52.318 & 0.0022 & 0.10 & $90 \mathrm{C}$ Aqueous Phase SDW \\
\hline BCC 157 & G3 & 4392 & 52.335 & 0.0019 & 0.09 & $90 \mathrm{C}$ Vapor Phase SDW \\
\hline
\end{tabular}




\section{Corrosion Rate Calculations for Crevice Specimens} For Rack Number : 30-1
Sample
Allov
Identification
Lag Time Surface Area
Weight Corrosion Rate Test Environment (hours) (sq cm) Loss (g) (microns/ycar)

\begin{tabular}{|c|c|c|c|c|c|c|}
\hline BCC 158 & G3 & 4392 & 52.182 & 0.0007 & 0.03 & $90 \mathrm{C}$ Vapor Phase SDW \\
\hline BCC 159 & G3 & 4392 & 52.548 & 0.0012 & 0.06 & 90 C Vapor Phase SDW \\
\hline BCC 160 & G3 & 4392 & 52.600 & 0.0015 & 0.07 & $90 \mathrm{C}$ Aqueous Phase SDW \\
\hline BCC 161 & G3 & 4392 & 52.383 & 0.0009 & 0.04 & $90 \mathrm{C}$ Aqueous Phase SDW \\
\hline BCC 162 & G3 & 4392 & 52.296 & 0.0014 & 0.06 & $90 \mathrm{C}$ Aqueous Phase SDW \\
\hline CCA 157 & $\mathrm{C} 4$ & 4392 & 52.156 & 0.0011 & 0.05 & $90 \mathrm{C}$ Vapor Phase SDW \\
\hline CCA 158 & $\mathrm{C} 4$ & 4392 & 52.319 & 0.0020 & 0.09 & $90 \mathrm{C}$ Vapor Phase SDW \\
\hline CCA 159 & $\mathrm{C}_{4}$ & 4392 & 52.235 & 0.0025 & 0.11 & $90 \mathrm{C}$ Vapor Phase SDW \\
\hline $\operatorname{CCA} 160$ & $\mathrm{C} 4$ & 4392 & 52.238 & 0.0004 & 0.02 & $90 \mathrm{C}$ Aqueous Phase SDW \\
\hline CCA 161 & $\mathrm{C} 4$ & 4392 & 51.670 & 0.0013 & 0.06 & $90 \mathrm{C}$ Aqueous Phase SDW \\
\hline CCA 162 & $\mathrm{C} 4$ & 4392 & 51.116 & 0.0018 & 0.08 & $90 \mathrm{C}$ Aqueous Phase SDW \\
\hline CCB 157 & $\mathrm{C} 4$ & 4392 & 51.432 & 0.0009 & 0.04 & 90 C Vapor Phase SDW \\
\hline CCB 158 & C4 & 4392 & 51.080 & 0.0020 & 0.09 & $90 \mathrm{C}$ Vapor Phase SDW \\
\hline CCB 159 & $\mathrm{C} 4$ & 4392 & 51.057 & 0.0015 & 0.07 & $90 \mathrm{C}$ Vapor Phase SDW \\
\hline ССВ 160 & $\mathrm{C4}$ & 4392 & 51.295 & 0.0011 & 0.05 & $90 \mathrm{C}$ Aqueous Phase SDW \\
\hline ССВ 161 & C4 & 4392 & 50.936 & 0.0014 & 0.06 & $90 \mathrm{C}$ Aqueous Phase SDW \\
\hline ССВ 162 & $\mathrm{C4}$ & 4392 & 51.012 & 0.0010 & 0.05 & $90 \mathrm{C}$ Aqueous Phase SDW \\
\hline DCA 157 & $\mathrm{C} 22$ & 4392 & 51.972 & 0.0013 & 0.06 & $90 \mathrm{C}$ Vapor Phase SDW \\
\hline DCA 158 & $\mathrm{C} 22$ & 4392 & 52.264 & 0.0014 & 0.06 & $90 \mathrm{C}$ Vapor Phase SOW \\
\hline
\end{tabular}




\section{Corrosion Rate Calculations for Crevice Specimens}

For Rack Number : 30-1

Sample Allov Lag Time Surface Area Weight Corrosion Rate Test Environment Identification

(sq cm) Loss (g) (microns/year)

\begin{tabular}{|c|c|c|c|c|c|c|}
\hline DCA 159 & $\mathrm{C} 22$ & 4392 & 52.002 & 0.0013 & 0.06 & $90 \mathrm{C}$ Vapor Phase SDW \\
\hline DCA 160 & $\mathrm{C} 22$ & 4392 & 51936 & 0.0023 & 0.10 & $90 \mathrm{C}$ Aqueous Phase SDW \\
\hline DCA 161 & $\mathrm{C} 22$ & 4392 & 51831 & 0.0027 & 0.12 & $90 \mathrm{C}$ Aqueous Phase SDW \\
\hline DCA 162 & $\mathrm{C} 22$ & 4392 & 51.708 & 0.0011 & 0.05 & $90 \mathrm{C}$ Aqueous Phase SDW \\
\hline DCB 157 & $\mathrm{C} 22$ & 4392 & 50.492 & 0.0016 & 0.07 & $90 \mathrm{C}$ Vapor Phase SDW \\
\hline DCB 158 & $\mathrm{C} 22$ & 4392 & 50.804 & 0.0017 & 0.08 & $90 \mathrm{C}$ Vapor Phase SDW \\
\hline DCB 159 & $\mathrm{C} 22$ & 4392 & 51.751 & 0.0011 & 0.05 & $90 \mathrm{C}$ Vapor Phase SDW \\
\hline DCB 160 & $\mathrm{C} 22$ & 4392 & 50.887 & 0.0014 & 0.06 & $90 \mathrm{C}$ Aqueous Phase SDW \\
\hline DCB 161 & $\mathrm{C} 22$ & 4392 & 51.689 & 0.0018 & 0.08 & $90 \mathrm{C}$ Aqueous Phase SDW \\
\hline DCB 162 & $\mathrm{C} 22$ & 4392 & 50.757 & 0.0009 & 0.04 & $90 \mathrm{C}$ Aqueous Phase SDW \\
\hline ECA 157 & Ti Grade 12 & 4392 & 52.182 & 0.0899 & 7.76 & $90 \mathrm{C}$ Vapor Phase SDW \\
\hline ECA 158 & Ti Grade 12 & 4392 & 52.574 & 0.0911 & 7.80 & $90 \mathrm{C}$ Vapor Phase SDW \\
\hline ECA 159 & Ti Grade 12 & 4392 & 52.423 & 0.1056 & 9.07 & 90 C Vapor Phase SDW \\
\hline ECA 160 & Ti Grade 12 & 4392 & 52.163 & 0.0784 & 6.77 & $90 \mathrm{C}$ Aqueous Phase SDW \\
\hline ECA 161 & $\pi$ Grade 12 & 4392 & 52.581 & 0.0804 & 6.88 & $90 \mathrm{C}$ Aqueous Phase SDW \\
\hline ECA 162 & Ti Grade 12 & 4392 & 51.541 & 0.0851 & 7.43 & $90 \mathrm{C}$ Aqueous Phase SDW \\
\hline ECD 157 & Ti Grade 12 & 4392 & 53.754 & 0.1051 & 8.80 & $90 \mathrm{C}$ Vapor Phase SDW \\
\hline ECD 158 & Ti Grade 12 & 4392 & 53.950 & 0.1011 & 8.44 & 90 C Vapor Phase SOW \\
\hline ECD 159 & Ti Grade 12 & 4392 & 53.774 & 0.0747 & 6.25 & $90 \mathrm{C}$ Vapor Phase SDW \\
\hline
\end{tabular}




\section{Corrosion Rate Calculations for Crevice Specimens}

For Rack Number : 30-1

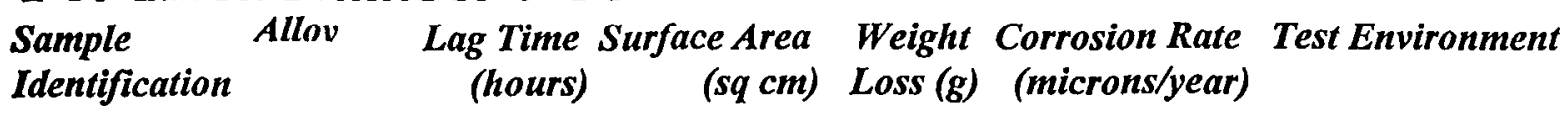

\begin{tabular}{|c|c|c|c|c|c|c|}
\hline ECD 160 & Ti Grade 12 & 4392 & 53.858 & 0.0683 & 5.71 & $90 \mathrm{C}$ Aqueous Phase SDW \\
\hline ECD 161 & Ti Grade 12 & 4392 & 53.695 & 0.0746 & 6.26 & $90 \mathrm{C}$ Aqueous Phase SDW \\
\hline ECD 162 & Ti Grade 12 & 4392 & 53.832 & 0.0793 & 6.63 & $90 \mathrm{C}$ Aqueous Phase SDW \\
\hline FCA 157 & $\pi$ Grade 16 & 4392 & 52.918 & 0.1595 & 13.30 & $90 \mathrm{C}$ Vapor Phase SDW \\
\hline FCA 158 & Ti Grade 16 & 4392 & 52.230 & 0.0832 & 7.03 & 90 C Vapor Phase SDW \\
\hline FCA 159 & Ti Grade 16 & 4392 & 52.682 & 0.0816 & 6.83 & $90 \mathrm{C}$ Vapor Phase SDW \\
\hline FCA 160 & Ti Grade 16 & 4392 & 53.027 & 0.0569 & 4.73 & $90 \mathrm{C}$ Aqueous Phase SDW \\
\hline FCA 161 & Ti Grade 16 & 4392 & 52.826 & 0.0632 & 5.28 & $90 \mathrm{C}$ Aqueous Phase SDW \\
\hline FCA 162 & Ti Grade 16 & 4392 & 52.880 & 0.0684 & 5.71 & $90 \mathrm{C}$ Aqueous Phase SDW \\
\hline FCE 157 & Ti Grade 16 & 4392 & 51.450 & 0.1385 & 11.88 & $90 \mathrm{C}$ Vapor Phase SDW \\
\hline FCE 158 & Ti Grade 16 & 4392 & 51.201 & 0.0987 & 8.51 & $90 \mathrm{C}$ Vapor Phase SDW \\
\hline FCE 159 & Ti Grade 16 & 4392 & 51.347 & 0.0595 & 5.11 & $90 \mathrm{C}$ Vapor Phase SDW \\
\hline FCE 160 & Ti Grade 16 & 4392 & 51.540 & 0.0724 & 6.20 & $90 \mathrm{C}$ Aqueous Phase SDW \\
\hline FCE 161 & Ti Grade 16 & 4392 & 51.849 & 0.0608 & 5.17 & $90 \mathrm{C}$ Aqueous Phase SDW \\
\hline FCE 162 & Ti Grade 16 & 4392 & 51.807 & 0.0704 & 6.00 & $90 \mathrm{C}$ Aqueous Phase SDW \\
\hline LCA 157 & Inconel 625 & 4392 & 52.133 & 0.0004 & 0.02 & $90 \mathrm{C}$ Vapor Phase SDW \\
\hline $\operatorname{LCA} 158$ & Inconel 625 & 4392 & 52.468 & 0.0003 & 0.01 & $90 \mathrm{C}$ Vapor Phase SDW \\
\hline LCA 159 & Inconel 625 & 4392 & 52.387 & 0.0000 & 0.00 & $90 \mathrm{C}$ Vapor Phase SDW \\
\hline LCA 160 & Inconel 625 & 4392 & 52.334 & 0.0003 & 0.01 & $90 \mathrm{C}$ Aqueous Phase SDW \\
\hline
\end{tabular}




\section{Corrosion Rate Calculations for Crevice Specimens}

\section{For Rack Number : 30-1}

$\begin{array}{lllll}\text { Sample Allov } & \text { Lag Time Surface Area Weight Corrosion Rate Test Environment } \\ \text { Identification } & \text { (hours) } & \text { (sq cm) } & \text { Loss }(g) & \text { (microns/year) }\end{array}$

\begin{tabular}{llccccc}
\hline LCA 161 & Inconel 625 & 4392 & 52189 & 0.0014 & 0.06 & $90 \mathrm{C}$ Aqueous Phase SDW \\
LCA 162 & Inconel 625 & 4392 & 52.250 & 0.0007 & 0.03 & 90 C Aqueous Phase SDW \\
LCJ 157 & Inconel 625 & 4392 & 51.615 & 0.0004 & 0.02 & $90 \mathrm{C}$ Vapor Phase SDW \\
LCJ 158 & Inconel 625 & 4392 & 51.420 & 0.0018 & 0.08 & $90 \mathrm{C}$ Vapor Phase SDW \\
LCJ 159 & Inconel 625 & 4392 & 51.429 & 0.0004 & 0.02 & $90 \mathrm{C}$ Vapor Phase SDW \\
LCJ 160 & Inconel 625 & 4392 & 50.066 & 0.0001 & 0.00 & $90 \mathrm{C}$ Aqueous Phase SDW \\
LCJ 161 & Inconel 625 & 4392 & 50.448 & 0.0005 & 0.02 & $90 \mathrm{C}$ Aqueous Phase SDW \\
LCJ 162 & Inconel 625 & 4392 & 52.062 & 0.0016 & 0.07 & $90 \mathrm{C}$ Aqueous Phase SDW
\end{tabular}




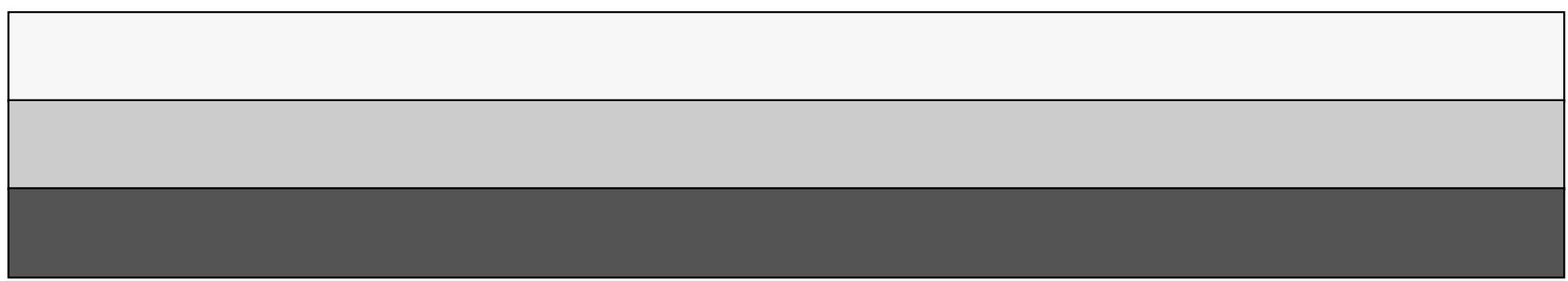

